\title{
PROPRIÉTÉS DE MAXIMALITÉ CONCERNANT UNE REPRÉSENTATION DÉFINIE PAR LUSZTIG
}

\author{
J.-L. WALDSPURGER
}

RÉsumÉ. Let $\lambda$ be a symplectic partition, denote $\operatorname{Jord}^{b p}(\lambda)$ the set of even positive integers which appear in $\lambda$, and let a map $\epsilon: \operatorname{Jord}^{b p}(\lambda) \rightarrow\{ \pm 1\}$. The generalized Springer's correspondence associates to $(\lambda, \epsilon)$ an irreducible representation $\rho(\lambda, \epsilon)$ of some Weyl group. We can also define a representation $\rho(\lambda, \epsilon)$ of the same Weyl group, in general reducible. Roughly speaking, $\rho(\lambda, \epsilon)$ is the representation of the Weyl group in the top cohomology group of some variety and $\underline{\rho}(\lambda, \epsilon)$ is the representation in the sum of all the cohomology groups of the same variety. The representation $\rho$ decomposes as a direct sum of $\rho\left(\lambda^{\prime}, \epsilon^{\prime}\right)$ with some multiplicities, where $\left(\lambda^{\prime}, \epsilon^{\prime}\right)$ describes the set of pairs similar to $(\lambda, \epsilon)$. It is well know that $(\lambda, \epsilon)$ appears in this decomposition with multiplicity one and is minimal in this decomposition. That is, if $\left(\lambda^{\prime}, \epsilon^{\prime}\right)$ appears, we have $\lambda^{\prime}>\lambda$ or $\left(\lambda^{\prime}, \epsilon^{\prime}\right)=(\lambda, \epsilon)$. Assuming that $\lambda$ has only even parts, we prove that there exists also a maximal pair $\left(\lambda^{\max }, \epsilon^{\max }\right)$. That is, $\left(\lambda^{\max }, \epsilon^{\max }\right)$ appears with positive multiplicity (in fact one) and, if $\left(\lambda^{\prime}, \epsilon^{\prime}\right)$ appears, we have $\lambda^{\max }>\lambda^{\prime}$ or $\left(\lambda^{\prime}, \epsilon^{\prime}\right)=\left(\lambda^{\max }, \epsilon^{\max }\right)$.

\section{INTRODUCTION}

Soit $N \geq 1$ un entier. On note $\mathcal{P}(2 N)$ l'ensemble des partitions de $2 N$, c'est-àdire celui des suites d'entiers $\lambda=\left(\lambda_{1} \geq \lambda_{2} \geq \ldots \geq \lambda_{r} \geq 0\right)$ telles que $S(\lambda)=2 N$, où $S(\lambda)=\lambda_{1}+\ldots+\lambda_{r}$. Deux suites sont identifiées si elles ne diffèrent que par des termes nuls. Pour une telle partition et pour un entier $i \geq 1$, on note $\operatorname{mult}_{\lambda}(i)$ le nombre d'indices $j$ tels que $\lambda_{j}=i$. Notons $\mathcal{P}^{s y m p}(2 N)$ le sous-ensemble des partitions symplectiques, c'est-à-dire celles telles que pour tout entier $i \geq 1$ impair, mult $_{\lambda}(i)$ est pair. Pour $\lambda \in \mathcal{P}^{s y m p}(2 N)$, notons $\operatorname{Jord}^{b p}(\lambda)$ l'ensemble des entiers $i \geq 2$ pairs tels que mult $_{\lambda}(i) \neq 0$. On note $\mathcal{P}^{\boldsymbol{s y m p}}(2 N)$ l'ensemble des couples $(\lambda, \epsilon)$ où $\lambda \in \mathcal{P}^{\text {symp }}(2 N)$ et $\epsilon \in\{ \pm 1\}^{\text {Jord }}{ }^{b p}(\lambda)$.

Pour tout entier $n \in \mathbb{N}$, notons $W_{n}$ le groupe de Weyl d'un système de racines de type $B_{n}$ ou $C_{n}$, avec la convention $W_{0}=\{1\}$. Notons $\mathcal{W}_{N}$ l'ensemble des couples $(k, \rho)$ où $k$ est un entier tel que $k \geq 0$ et $k(k+1) \leq 2 N$ et $\rho$ est une représentation irréductible de $W_{N-k(k+1) / 2}$. La correspondance de Springer généralisée, définie par Lusztig, est une bijection $(\lambda, \epsilon) \mapsto(k(\lambda, \epsilon), \rho(\lambda, \epsilon))$ de $\mathcal{P}^{\text {symp }}(2 N)$ sur $\mathcal{W}_{N}$.

Il est bien connu que $\mathcal{P}^{s y m p}(2 N)$ paramètre les orbites unipotentes dans le groupe symplectique $S p(2 N ; \mathbb{C})$. L'ensemble $\mathcal{P}^{\boldsymbol{s y m p}}(2 N)$ paramètre les couples $(C, \mathcal{E})$ formés d'une orbite unipotente $C$ et d'un système local $S p(2 N ; \mathbb{C})$-équivariant irréductible sur $C$. On note $\left(C_{\lambda, \epsilon}, \mathcal{E}_{\lambda, \epsilon}\right)$ le couple paramétré par $(\lambda, \epsilon) \in \mathcal{P}^{s \boldsymbol{y} m \boldsymbol{p}}(2 N)$. Pour un tel couple $\left(C^{\prime}, \mathcal{E}^{\prime}\right)$, on définit le prolongement d'intersection $I C\left(\bar{C}^{\prime}, \mathcal{E}^{\prime}\right)$, qui est supporté par la fermeture $\bar{C}^{\prime}$ de $C^{\prime}$, et ses faisceaux de cohomologie $\mathcal{H}^{m} I C\left(\bar{C}^{\prime}\right.$,

Received by the editors June 8, 2018, and, in revised form, August 22, 2019.

2010 Mathematics Subject Classification. 05E10, 20 C30. 
$\left.\mathcal{E}^{\prime}\right)$. Lusztig a montré que ces derniers sont nuls si $m$ est impair. Si $C$ est une autre orbite unipotente, la restriction de $\mathcal{H}^{2 m} I C\left(\bar{C}^{\prime}, \mathcal{E}^{\prime}\right)$ à $C$ est somme directe de systèmes locaux $S p(2 N ; \mathbb{C})$-équivariants irréductibles. Si $\mathcal{E}$ est un tel système local sur $C$, on note $\mathfrak{m u l t}\left(C, \mathcal{E} ; C^{\prime}, \mathcal{E}^{\prime}\right)$ la multiplicité avec laquelle intervient $\mathcal{E}$ dans la restriction à $C$ de $\oplus_{m \in \mathbb{Z}} \mathcal{H}^{2 m} I C\left(\bar{C}^{\prime}, \mathcal{E}^{\prime}\right)$. Soient $(\lambda, \epsilon)$ et $\left(\lambda^{\prime}, \epsilon^{\prime}\right)$ les éléments de $\mathcal{P}^{s y m p}(2 N)$ paramétrant respectivement $(C, \mathcal{E})$ et $\left(C^{\prime}, \mathcal{E}^{\prime}\right)$. On pose $\mathfrak{m u l t}\left(\lambda, \epsilon ; \lambda^{\prime}, \epsilon^{\prime}\right)=\mathfrak{m u l t}(C, \mathcal{E}$; $\left.C^{\prime}, \mathcal{E}^{\prime}\right)$. Supposons que cette multiplicité soit non nulle. Il est clair qu'alors $\lambda \leq \lambda^{\prime}$ pour l'ordre usuel des partitions. Lusztig a prouvé que $k(\lambda, \epsilon)=k\left(\lambda^{\prime}, \epsilon^{\prime}\right)$.

Partons maintenant de $(\lambda, \epsilon) \in \mathcal{P}^{\boldsymbol{s y m p}}(2 N)$. Posons pour simplifier $k=k(\lambda, \epsilon)$. On définit la représentation $\underline{\rho}(\lambda, \epsilon)$ de $W_{N-k(k+1) / 2}$ par

$$
\underline{\rho}(\lambda, \epsilon)=\oplus_{\left(\lambda^{\prime}, \epsilon^{\prime}\right) \in \mathcal{P}^{s y m p}(2 N)} \mathfrak{m u l t}\left(\lambda, \epsilon ; \lambda^{\prime}, \epsilon^{\prime}\right) \rho\left(\lambda^{\prime}, \epsilon^{\prime}\right) .
$$

Les coefficients $\mathfrak{m u l t}\left(\lambda, \epsilon ; \lambda^{\prime}, \epsilon^{\prime}\right)$ interviennent de façon essentielle dans de nombreux travaux concernant les groupes réductifs finis, la première occurence étant peut-être [1] theorem 24.4. Ce qui nous concerne plus directement est que la représentation $\rho(\lambda, \epsilon)$ contrôle les restrictions aux sous-groupes parahoriques des représentations de réduction unipotente des groupes $p$-adiques (voir [2] et [5] proposition 5.2). Or on sait peu de choses sur cette représentation. Il nous semble qu'en général, outre ce que l'on a déjà dit ci-dessus $\left(\mathfrak{m u l t}\left(\lambda, \epsilon ; \lambda^{\prime}, \epsilon^{\prime}\right) \neq 0\right.$ entraîne $\left.k\left(\lambda^{\prime}, \epsilon^{\prime}\right)=k(\lambda, \epsilon)\right)$, on ne connaît que la propriété de minimalité

$\mathfrak{m u l t}\left(\lambda, \epsilon ; \lambda^{\prime}, \epsilon^{\prime}\right) \neq 0$ entraîne $\lambda^{\prime}>\lambda$ ou $\left(\lambda^{\prime}, \epsilon^{\prime}\right)=(\lambda, \epsilon)$; dans ce dernier cas $\mathfrak{m u l t}(\lambda, \epsilon ; \lambda, \epsilon)=1$.

Le but de l'article est de démontrer que, sous une hypothèse de parité sur $\lambda$, la représentation $\rho(\lambda, \epsilon)$ possède aussi une propriété de maximalité. Précisément, nous démontrerons le théorème suivant.

Théorème. Soit $(\lambda, \epsilon) \in \mathcal{P}^{\text {symp }}(2 N)$. Supposons que $\lambda$ n'a que des termes pairs. Alors il existe un unique élément $\left(\lambda^{\max }, \epsilon^{\max }\right) \in \mathcal{P}^{\text {symp }}(2 N)$ vérifiant les propriétés suivantes :

(i) $\mathfrak{m u l t}\left(\lambda, \epsilon ; \lambda^{\max }, \epsilon^{\max }\right)=1$;

(ii) pour tout élément $\left(\lambda^{\prime}, \epsilon^{\prime}\right) \in \mathcal{P}^{\text {symp }}(2 N)$ tel que $\mathfrak{m u l t}\left(\lambda, \epsilon ; \lambda^{\prime}, \epsilon^{\prime}\right) \neq 0$, on a $\lambda^{\prime}<\lambda^{\max }$ ou $\left(\lambda^{\prime}, \epsilon^{\prime}\right)=\left(\lambda^{\max }, \epsilon^{\max }\right)$.

En fait, notre méthode permet une certaine latitude sur l'ordre choisi pour comparer les partitions, ou plus exactement les éléments de $\mathcal{P}^{\boldsymbol{s y} \boldsymbol{y} \boldsymbol{p}}(2 N)$. Pour tout entier $n \in \mathbb{N}$, notons simplement sgn le caractère signature de $W_{n}$. Pour $(\lambda, \epsilon) \in$ $\mathcal{P}^{s y m p}(2 N)$, notons $\left({ }^{s} \lambda,{ }^{s} \epsilon\right)$ l'élément de $\mathcal{P}^{s y m p}(2 N)$ tel que $k\left({ }^{s} \lambda,{ }^{s} \epsilon\right)=k(\lambda, \epsilon)$ et $\rho\left({ }^{s} \lambda,{ }^{s} \epsilon\right)=\operatorname{sgn} \otimes \rho(\lambda, \epsilon)$ (la notation ne doit pas prêter à confusion : ${ }^{s} \lambda$, resp. ${ }^{s} \epsilon$, ne dépend pas seulement de $\lambda$, resp. $\epsilon$, mais du couple $(\lambda, \epsilon))$. En général, cette opération n'est pas décroissante pour l'ordre usuel des partitions, c'est-à-dire que, pour $(\lambda, \epsilon),\left(\lambda^{\prime}, \epsilon^{\prime}\right) \in \mathcal{P}^{s y m p}(2 N)$, la relation $\lambda \leq \lambda^{\prime}$ n'entraîne pas ${ }^{s} \lambda^{\prime} \leq{ }^{s} \lambda$. Toutefois, nous démontrerons le théorème suivant.

Théorème. Soit $(\lambda, \epsilon) \in \mathcal{P}^{\text {symp }}(2 N)$. Supposons que $\lambda$ n'a que des termes pairs. Alors il existe un unique élément $\left(\lambda^{\text {min }}, \epsilon^{\text {min }}\right) \in \mathcal{P}^{\text {symp }}(2 N)$ vérifiant les propriétés suivantes :

(i) $\mathfrak{m u l t}\left(\lambda, \epsilon ;{ }^{s} \lambda^{\min },{ }^{s} \epsilon^{\min }\right)=1$; 
(ii) pour tout élément $\left(\lambda^{\prime}, \epsilon^{\prime}\right) \in \mathcal{P}^{s y m p}(2 N)$ tel que $\mathfrak{m u l t}\left(\lambda, \epsilon ;{ }^{s} \lambda^{\prime},{ }^{s} \epsilon^{\prime}\right) \neq 0$, on a $\lambda^{\text {min }}<\lambda^{\prime}$ ou $\left(\lambda^{\prime}, \epsilon^{\prime}\right)=\left(\lambda^{\text {min }}, \epsilon^{\text {min }}\right)$.

De plus, on $a\left({ }^{s} \lambda^{\min },{ }^{s} \epsilon^{\min }\right)=\left(\lambda^{\max }, \epsilon^{\max }\right)$.

Dans un article ultérieur, nous utiliserons ce résultat pour prouver que certaines représentations (très particulières) de groupes $p$-adiques admettent un front d'onde.

Le couple $\left(\lambda^{\max }, \epsilon^{\max }\right)$ peut se calculer explicitement. Par contre, nous n'avons pas trouvé de formule "simple" pour calculer le couple $\left(\lambda^{\min }, \epsilon^{\text {min }}\right)$ (il peut évidemment se calculer à partir de $\left(\lambda^{\max }, \epsilon^{\max }\right)$ et des formules explicites pour la représentation de Springer généralisée, mais ce calcul n'est pas "simple").

Les preuves des théorèmes sont purement combinatoires. Comme on le sait, les représentations irréductibles d'un groupe $W_{n}$ sont paramétrées par les couples $(\alpha, \beta)$ de partitions telles que $S(\alpha)+S(\beta)=n$. Une multiplicité mult $\left(\lambda, \epsilon ; \lambda^{\prime}, \epsilon^{\prime}\right)$ peut se récrire $\mathfrak{m u l t}\left(\alpha, \beta ; \alpha^{\prime}, \beta^{\prime}\right)$ où $(\alpha, \beta)$ paramètre $\rho(\lambda, \epsilon)$ et $\left(\alpha^{\prime}, \beta^{\prime}\right)$ paramètre $\rho\left(\lambda^{\prime}, \epsilon^{\prime}\right)$. Shoji a développé une théorie des fonctions symétriques associées à des couples de partitions. En particulier, à l'aide d'un couple $(\alpha, \beta)$ et d'un ordre $<$ sur l'ensemble d'indices de ce couple, il définit un polynôme du type polynôme de Kostka. Pour un autre couple $\left(\alpha^{\prime}, \beta^{\prime}\right)$, on peut définir la "multiplicité" mult $\left(\alpha, \beta,<; \alpha^{\prime}, \beta^{\prime}\right)$ de $\left(\alpha^{\prime}, \beta^{\prime}\right)$ dans ce polynôme. Sous certaines hypothèses sur $(\lambda, \epsilon)$ (la paire associée à $(\alpha, \beta))$ et pour un ordre $<$ bien choisi, Shoji prouve que mult $(\alpha, \beta,<$ $\left.; \alpha^{\prime}, \beta^{\prime}\right)=\mathfrak{m u l t}\left(\alpha, \beta ; \alpha^{\prime}, \beta^{\prime}\right)$ pour tout $\left(\alpha^{\prime}, \beta^{\prime}\right)$. Nous généraliserons un peu ce résultat en 4.2 : il est valable pourvu que $\lambda$ n'ait que des termes pairs. Les assertions des théorèmes ci-dessus deviennent des assertions concernant les paires $\left(\alpha^{\prime}, \beta^{\prime}\right)$ telles que $\operatorname{mult}\left(\alpha, \beta,<; \alpha^{\prime}, \beta^{\prime}\right) \neq 0$. Et la définition de ces multiplicités est purement combinatoire.

Au premier paragraphe, on considère un couple de partitions $(\alpha, \beta)$ et un ordre $<$ sur l'ensemble d'indices de ce couple. On définit des ensembles $P(\alpha, \beta,<)$ et $P_{A, B ; s}(\alpha, \beta,<)$ formés de couples de partitions $(\nu, \mu)$ déduits de $(\alpha, \beta)$ par une combinatoire simple. On montre au paragraphe 3 que ces couples sont précisément ceux qui interviennent dans le polynôme de Shoji associé à $(\alpha, \beta,<)$ avec des propriétés de maximalité du type de celles intervenant dans les théorèmes ci-dessus. Entretemps, le paragraphe 2 est consacré à prouver divers lemmes techniques concernant ces ensembles $P(\alpha, \beta,<)$ et $P_{A, B ; s}(\alpha, \beta,<)$. Ils peuvent être négligés en première lecture et même en deuxième. Au paragraphe 4 , on étend comme on l'a dit le théorème de Shoji et on en déduit les théorèmes, à l'aide des résultats de maximalité prouvés au paragraphe 3 . La description explicite du couple $\left(\lambda^{\max }, \epsilon^{\max }\right)$ est donnée au paragraphe 5 .

\section{DÉFINITIONS}

1.1. Suites de nombres. On note $\mathcal{R}$ l'ensemble des suites infinies $\lambda=\left(\lambda_{1}, \lambda_{2}, \ldots\right)$ décroissantes de nombres réels qui vérifient la propriété suivante : pour tout réel $r$, il n'existe qu'un nombre fini de termes $\lambda_{i} \geq r$. Pour $\lambda \in \mathcal{R}$ et pour $r \in \mathbb{R}$, on note mult $_{\lambda}(r)$ le nombre d'indices $i \geq 1$ tels que $\lambda_{i}=r$. Pour $c \in \mathbb{N}$, on pose $S_{c}(\lambda)=\lambda_{1}+\ldots+\lambda_{c}$. On munit $\mathcal{R}$ de l'ordre défini par : $\lambda \leq \lambda^{\prime}$ si et seulement si $S_{c}(\lambda) \leq S_{c}\left(\lambda^{\prime}\right)$ pour tout $c \in \mathbb{N}$. Pour $\lambda, \lambda^{\prime} \in \mathcal{R}$, on note $\lambda+\lambda^{\prime}$ la suite $\left(\lambda_{1}+\right.$ $\left.\lambda_{1}^{\prime}, \lambda_{2}+\lambda_{2}^{\prime}, \ldots\right)$. Elle appartient à $\mathcal{R}$. On note $\lambda \sqcup \lambda^{\prime}$ l'unique élément de $\mathcal{R}$ tel que mult $_{\lambda \sqcup \lambda^{\prime}}(r)=$ mult $_{\lambda}(r)+$ mult $_{\lambda^{\prime}}(r)$ pour tout $r \in \mathbb{R}$. 
Notons $\mathcal{R}_{f}$ l'ensemble des suites finies $\lambda=\left(\lambda_{1}, \ldots, \lambda_{l}\right)$ décroissantes de nombres réels. On note $l(\lambda)$ la longueur $l$ de la suite. Les définitions ci-dessus valent pour les éléments de $\mathcal{R}_{f}$ :

la fonction mult $_{\lambda}$;

la somme partielle $S_{c}(\lambda)$ pourvu que $c \leq l(\lambda)$; on pose $S(\lambda)=S_{l(\lambda)}(\lambda)$;

l'ordre $\lambda \leq \lambda^{\prime}$ pourvu que $l(\lambda)=l\left(\lambda^{\prime}\right)$.

Pour $\lambda \in \mathcal{R}$ et $\lambda^{\prime} \in \mathcal{R}_{f}$, on définit comme ci-dessus la suite $\lambda \sqcup \lambda^{\prime} \in \mathcal{R}$.

On appelle partition une suite infinie $\lambda=\left(\lambda_{1}, \lambda_{2}, \ldots\right)$ décroissante de nombres entiers positifs ou nuls qui ne contient qu'un nombre fini de termes non nuls. On note $t(\lambda)$ ce nombre de termes non nuls. Pour tout entier $n \geq t(\lambda)$, on identifiera si besoin est la partition $\lambda$ à la suite finie $\left(\lambda_{1}, \ldots, \lambda_{n}\right)$. On note $\mathcal{P}$ l'ensemble des partitions. On note $\emptyset$ la partition dont tous les termes sont nuls. Les définitions ci-dessus valent pour les éléments de $\mathcal{P}$ :

la fonction mult $_{\lambda}$, dont on restreint l'ensemble de définition à $\mathbb{N}_{>0}=\mathbb{N}-\{0\}$;

la somme partielle $S_{c}(\lambda)$; on pose $S(\lambda)=S_{t(\lambda)}(\lambda)$;

l'ordre $\lambda \leq \lambda^{\prime}$

la somme $\lambda+\lambda^{\prime}$ et la réunion $\lambda \sqcup \lambda^{\prime}$.

Pour $\lambda \in \mathcal{R}$ et $\mu \in \mathcal{P}$, on définit comme ci-dessus la suite $\lambda+\mu \in \mathcal{R}$.

Lemme. Soient $\lambda, \lambda^{\prime}, \mu, \mu^{\prime} \in \mathcal{R}$.

(i) Supposons $\lambda \leq \lambda^{\prime}$ et $\mu \leq \mu^{\prime}$; alors $\lambda \sqcup \mu \leq \lambda^{\prime} \sqcup \mu^{\prime}$.

(ii) Supposons $\mu=\mu^{\prime}$. Alors $\lambda \leq \lambda^{\prime}$ si et seulement si $\lambda \sqcup \mu \leq \lambda^{\prime} \sqcup \mu$.

(iii) Supposons $\lambda \leq \lambda^{\prime}$ et $\mu \leq \mu^{\prime}$; alors $\lambda \sqcup \mu=\lambda^{\prime} \sqcup \mu^{\prime}$ si et seulement si $\lambda=\lambda^{\prime}$ et $\mu=\mu^{\prime}$.

Les mêmes conclusions valent si l'on suppose

$\lambda, \lambda^{\prime} \in \mathcal{R}$ et $\mu, \mu^{\prime} \in \mathcal{R}_{f}$ avec $l(\mu)=l\left(\mu^{\prime}\right) ;$

ou $\mu, \mu^{\prime} \in \mathcal{R}$ et $\lambda, \lambda^{\prime} \in \mathcal{R}_{f}$ avec $l(\lambda)=l\left(\lambda^{\prime}\right)$;

ou $\lambda, \lambda^{\prime}, \mu, \mu^{\prime} \in \mathcal{P}$.

Preuve. Remarquons d'abord que, par définition de la réunion, pour $c \in \mathbb{N}, S_{c}(\lambda \sqcup \mu)$ est le sup de $S_{a}(\lambda)+S_{b}(\mu)$ quand $(a, b)$ parcourt les couples d'entiers positifs ou nuls tels que $a+b=c$.

Démontrons (i). Soient $a, b$ tels que $S_{c}(\lambda \sqcup \mu)=S_{a}(\lambda)+S_{b}(\mu)$. Puisque $\lambda \leq \lambda^{\prime}$ et $\mu \leq \mu^{\prime}$, on a $S_{a}(\lambda) \leq S_{a}\left(\lambda^{\prime}\right)$ et $S_{b}(\mu) \leq S_{b}\left(\mu^{\prime}\right)$, d'où

$$
S_{c}(\lambda \sqcup \mu) \leq S_{a}\left(\lambda^{\prime}\right)+S_{b}\left(\mu^{\prime}\right) \leq S_{c}\left(\lambda^{\prime} \sqcup \mu^{\prime}\right) .
$$

Cela étant vrai pour tout $c$, on conclut $\lambda \sqcup \mu \leq \lambda^{\prime} \sqcup \mu^{\prime}$, d'où (i).

Démontrons (ii). L'assertion "seulement si" résulte de (i). Supposons $\lambda \sqcup \mu \leq$ $\lambda^{\prime} \sqcup \mu$. Soit $c \geq 1$ un entier. Notons $d$ le plus grand entier positif tel que $\mu_{d}>\lambda_{c}^{\prime}$ si un tel entier existe et $d=0$ sinon. On vérifie que $S_{c+d}\left(\lambda^{\prime} \sqcup \mu\right)=S_{c}\left(\lambda^{\prime}\right)+S_{d}(\mu)$. On a $S_{c}(\lambda)+S_{d}(\mu) \leq S_{c+d}(\lambda+\mu)$ d'après la remarque débutant la preuve et $S_{c+d}(\lambda+\mu) \leq S_{c+d}\left(\lambda^{\prime}+\mu\right)$ puisque $\lambda \sqcup \mu \leq \lambda^{\prime} \sqcup \mu$. D'où

$$
S_{c}(\lambda)+S_{d}(\mu) \leq S_{c}\left(\lambda^{\prime}\right)+S_{d}(\mu)
$$

puis $S_{c}(\lambda) \leq S_{c}\left(\lambda^{\prime}\right)$. Cela étant vrai pour tout $c$, on conclut $\lambda \leq \lambda^{\prime}$, ce qui démontre (ii).

Démontrons (iii). L'assertion "si" est évidente. Supposons $\lambda \sqcup \mu=\lambda^{\prime} \sqcup \mu^{\prime}$. D'après (i), on a

$$
\lambda \sqcup \mu \leq \lambda \sqcup \mu^{\prime} \leq \lambda^{\prime} \sqcup \mu^{\prime} .
$$


Les deux termes extrêmes étant égaux, ces inégalités sont des égalités. A fortiori $\lambda^{\prime} \sqcup \mu^{\prime} \leq \lambda \sqcup \mu^{\prime}$ et $\lambda \sqcup \mu^{\prime} \leq \lambda \sqcup \mu$. En appliquant (ii), on obtient $\lambda^{\prime} \leq \lambda$ et $\mu^{\prime} \leq \mu$. Puisque les inégalités opposées sont vraies par hypothèse, ce sont des égalités, ce qui démontre (iii).

Les variantes du lemme se démontrent de la même façon.

1.2. Ordres sur les ensembles d'indices . Soient $\alpha, \beta \in \mathcal{P}$. Considérons des entiers $n \geq t(\alpha), m \geq t(\beta)$ et identifions $\alpha$, resp. $\beta$, à une suite de longueur $n$, resp. $m$ (on peut avoir $n=0$ ou $m=0$ ). Pour différencier les ensembles d'indices de $\alpha$ et $\beta$, on considère que celui de $\alpha$ est l'ensemble des $(i, 0)$ pour $i=1, \ldots, n$ et que celui de $\beta$ est l'ensemble des $(i, 1)$ pour $i=1, \ldots, m$. Notons $I$ la réunion de ces ensembles d'indices. Soit $<$ un ordre total sur $I$ induisant l'ordre naturel sur chacun des sous-ensembles d'indices de $\alpha$ et $\beta$ (c'est-à-dire que, pour $e=0$ ou 1 , $(i, e)<(j, e)$ si et seulement si $i<j)$. Appelons le triplet $(n, m,<)$ un ordre sur l'ensemble d'indices de $(\alpha, \beta)$.

Considérons deux tels ordres $(n, m,<)$ et $\left(n^{\prime}, m^{\prime},<^{\prime}\right)$. Disons qu'ils sont équivalents si et seulement s'ils vérifient les conditions suivantes :

soit $i \geq 1$ tel que $\alpha_{i}>0$ (ce qui implique $i \leq n$ et $i \leq n^{\prime}$ puisque $n, n^{\prime} \geq t(\alpha)$ par hypothèse) ; alors l'ensemble des $j \in\{1, \ldots, m\}$ tels que $(j, 1)<(i, 0)$ est égal à celui des $j \in\left\{1, \ldots, m^{\prime}\right\}$ tels que $(j, 1)<^{\prime}(i, 0)$;

soit $j \geq 1$ tel que $\beta_{j}>0$; alors l'ensemble des $i \in\{1, \ldots, n\}$ tels que $(i, 0)<(j, 1)$ est égal à celui des $i \in\left\{1, \ldots, n^{\prime}\right\}$ tels que $(i, 0)<^{\prime}(j, 1)$.

On voit que dans chaque classe d'équivalence, il y a un unique représentant "minimal" $(n, m,<)$ tel que $n$ et $m$ soient minimaux. On le note plus précisément $\left(n_{<}, m_{<},<\right)$. Notons $I_{<}$son ensemble d'indices et $\left(t_{<}, e_{<}\right)$le plus grand élément de cet ensemble. Les ordres équivalents sont les $\left(n^{\prime}, m^{\prime},<^{\prime}\right)$ tels que $n_{<} \leq n^{\prime}, m_{<} \leq m^{\prime}$, $<^{\prime}$ coïncide avec $<\operatorname{sur} I_{<},\left(t_{<}, e_{<}\right)<^{\prime}(i, 0)$ pour $i \in\left\{n_{<}+1, \ldots, n^{\prime}\right\}$ et $\left(t_{<}, e_{<}\right)<^{\prime}$ $(j, 1)$ pour $j \in\left\{m_{<}+1, \ldots, m^{\prime}\right\}$. Autrement dit, ils se déduisent de l'ordre minimal par une suite de l'une ou l'autre des opérations suivantes : on part d'un ordre $(n, m,<)$ et on le remplace par $\left(n+1, m,<^{\prime}\right)$, resp. $\left(n, m+1,<^{\prime}\right)$, en décidant que $<$ coïncide avec $<$ sur l'ensemble d'indices de $(n, m,<)$ et que $(n+1,0)$, resp. $(m+1,1)$, est l'élément maximal pour $<$ '.

Remarquons que si, par exemple $e_{<}=0$, on a $n_{<}=t(\alpha)$ ainsi qu'il résulte des définitions mais on peut avoir $m_{<}>t(\beta)$. Par exemple, pour $\alpha=\left(\alpha_{1}, \alpha_{2}, 0, \ldots\right)$ et $\beta=\left(\beta_{1}, 0 \ldots\right)$ avec $\alpha_{2}>0$ et $\beta_{1}>0$, l'ordre $(2,3,<)$ défini par

$$
(1,0)<(1,1)<(2,1)<(3,1)<(2,0)
$$

est un ordre minimal.

Dans la suite, on considère des classes d'équivalence d'ordres sur l'ensemble d'indices de $(\alpha, \beta)$. On notera simplement $<$ une telle classe. Lorsqu'on saura que les constructions effectuées ne dépendent que de cette classe d'équivalence, on supposera implicitement fixé un représentant $(n, m,<)$ de cette classe. Si on écrit une inégalité telle que $(1,0)<(1,1)$, on supposera implicitement que $n, m \geq 1$.

On utilisera souvent la propriété suivante :

(1) supposons $(1,0)<(1,1)$ et $m_{<}>0$; alors $n_{<}>0$.

En effet, si $\alpha_{1} \neq 0$, c'est clair. Sinon, on a $\alpha=\emptyset$. Alors l'hypothèse $m_{<}>0$ implique que $\beta \neq \emptyset$, donc $\beta_{1} \neq 0$. Puisque $(1,0)<(1,1)$, on a alors $n_{<}>0$ par définition de ce nombre. 
Soit $X$ un sous-ensemble fini de l'ensemble d'indices $\mathbb{N}_{>0}$ de $\alpha$. On définit une partition extraite $\alpha^{\prime}$ de $\alpha$ en supprimant les termes $\alpha_{i}$ pour $i \in X$ et en réindiçant les termes restants. Autrement dit, notons $\varphi_{X}: \mathbb{N}_{>0} \rightarrow \mathbb{N}_{>0}-X$ l'unique bijection croissante. On pose $\alpha_{i}^{\prime}=\alpha_{\varphi_{X}(i)}$. Soit $Y$ un sous-ensemble fini de l'ensemble d'indices $\mathbb{N}_{>0}$ de $\beta$. En utilisant cet ensemble, on définit de même une partition extraite $\beta^{\prime}$ de $\beta$. Considérons maintenant un ordre $(n, m,<)$ sur l'ensemble d'indices de $(\alpha, \beta)$. Notons $n^{\prime}$ le plus grand entier strictement positif tel que $\varphi_{X}\left(n^{\prime}\right) \leq n$ si un tel entier existe, $n^{\prime}=0$ sinon. On définit $m^{\prime}$ de façon symétrique. On vérifie que $n^{\prime} \geq t\left(\alpha^{\prime}\right), m^{\prime} \geq t\left(\beta^{\prime}\right)$. On munit l'ensemble d'indices $I^{\prime}$ de $\left(\alpha^{\prime}, \beta^{\prime}\right)$ de l'unique ordre $<^{\prime}$ induisant l'ordre naturel sur chacun des sous-ensembles d'indices de $\alpha^{\prime}$ et $\beta^{\prime}$ et tel que $(i, 0)<^{\prime}(j, 1)$ si et seulement si $\left(\varphi_{X}(i), 0\right)<\left(\varphi_{Y}(j), 1\right)$. On obtient un ordre $\left(n^{\prime}, m^{\prime},<^{\prime}\right)$ sur l'ensemble d'indices de $\left(\alpha^{\prime}, \beta^{\prime}\right)$. On vérifie que $n_{<^{\prime}}+m_{<^{\prime}} \leq n_{<}+m_{<}$ et que cette inégalité est stricte si $X \cap\left\{1, \ldots, n_{<}\right\}$ou $Y \cap\left\{1, \ldots, m_{<}\right\}$est non vide (si ces deux ensembles sont vides, $\alpha^{\prime}=\alpha$ et $\beta^{\prime}=\beta$ et l'ordre $\left(n^{\prime}, m^{\prime},<^{\prime}\right)$ est équivalent à l'ordre de départ).

Remplaçons maintenant $(n, m,<)$ par un ordre équivalent $(\tilde{n}, \tilde{m}, \tilde{<})$ et $X$ et $Y$ par des ensembles $\tilde{X}, \tilde{Y}$. Supposons que

$$
X \cap\left\{1, \ldots, n_{<}\right\}=\tilde{X} \cap\left\{1, \ldots, n_{<}\right\}, Y \cap\left\{1, \ldots, m_{<}\right\}=\tilde{Y} \cap\left\{1, \ldots, m_{<}\right\}
$$

On vérifie que les partitions extraites $\alpha^{\prime}$ et $\beta^{\prime}$ ne changent pas et que le nouvel ordre obtenu $\left(\tilde{n}^{\prime}, \tilde{m}^{\prime}, \tilde{<}^{\prime}\right)$ sur l'ensemble d'indices de $\left(\alpha^{\prime}, \beta^{\prime}\right)$ est équivalent à $\left(n^{\prime}, m^{\prime},<^{\prime}\right)$. On dira que la classe d'équivalence de $<^{\prime}$ est l'ordre induit par $<$. C'est toujours cet ordre que nous utiliserons et nous le noterons sans plus de commentaire conformément au couple de partitions extraites $\left(<^{\prime}\right.$ si, comme ici, ce couple est noté $\left(\alpha^{\prime}, \beta^{\prime}\right),<^{i}$ si le couple est noté $\left(\alpha^{i}, \beta^{i}\right)$ etc...). La formation de l'ordre induit est transitive au sens suivant. Soit $\left(\alpha^{\prime}, \beta^{\prime}\right)$ un couple de partitions extraites de $(\alpha, \beta)$ et soit $\left(\alpha^{\prime \prime}, \beta^{\prime \prime}\right)$ un couple de partitions extraites de $\left(\alpha^{\prime}, \beta^{\prime}\right)$. On construit l'ordre induit $<^{\prime}$ sur l'ensemble d'indices de $\left(\alpha^{\prime}, \beta^{\prime}\right)$, puis l'ordre induit $<^{\prime \prime}$ par $<^{\prime}$ sur l'ensemble d'indices de $\left(\alpha^{\prime \prime}, \beta^{\prime \prime}\right)$. Alors cet ordre $<^{\prime \prime}$ est aussi l'ordre induit par $<$ en considérant $\left(\alpha^{\prime \prime}, \beta^{\prime \prime}\right)$ comme un couple de partitions extraites de $(\alpha, \beta)$.

1.3. L'ensemble $P(\alpha, \beta,<)$. Pour la suite de la section, on fixe $\alpha, \beta \in \mathcal{P}$ et une classe d'équivalence d'ordre $<$ sur l'ensemble d'indices de $(\alpha, \beta)$.

Nous allons décrire deux procédés (a), resp. (b). Ils construisent un entier $\nu_{1}$, resp. $\mu_{1}$, un couple de partitions $\left(\alpha^{\prime}, \beta^{\prime}\right)$ et une classe d'équivalence d'ordre sur l'ensemble d'indices de $\left(\alpha^{\prime}, \beta^{\prime}\right)$.

Pour le procédé $(\mathrm{a})$, on fixe un représentant $(n, m,<)$ de l'ordre tel que $n \geq 1$. On pose $a_{1}=1$. On note $b_{1}$ le plus petit élément de $\{1, \ldots, m\}$ tel que $(1,0)<\left(b_{1}, 1\right)$ s'il en existe, puis $a_{2}$ le plus petit élément de $\{1, \ldots, n\}$ tel que $\left(b_{1}, 1\right)<\left(a_{2}, 0\right)$ s'il en existe, puis $b_{2}$ le plus petit élément de $\{1, \ldots, m\}$ tel que $\left(a_{2}, 0\right)<\left(b_{2}, 1\right)$ s'il en existe etc... On arrête le procédé quand il n'y a plus d'élément vérifiant l'inégalité requise. On pose $\nu_{1}=\alpha_{a_{1}}+\beta_{b_{1}}+\alpha_{a_{2}}+\beta_{b_{2}}+\ldots$. On note $\alpha^{\prime}$ la partition extraite de $\alpha$ en supprimant les termes d'indices $a_{1}, a_{2}, \ldots$ et $\beta^{\prime}$ la partition extraite de $\beta$ en supprimant les termes d'indices $b_{1}, b_{2}, \ldots$. Comme on l'a dit en 1.2, on munit l'ensemble d'indices de $\left(\alpha^{\prime}, \beta^{\prime}\right)$ de l'ordre $<^{\prime}$ induit de $(n, m,<)$. Il est clair que

(1) $\nu_{1}+S\left(\alpha^{\prime}\right)+S\left(\beta^{\prime}\right)=S(\alpha)+S(\beta)$. 
Vérifions que les objets que l'on vient de construire ne dépendent pas (à équivalence près en ce qui concerne les ordres) du représentant $(n, m,<)$ choisi. Introduisons un autre représentant $(\boldsymbol{n}, \boldsymbol{m},<)$ avec $\boldsymbol{n} \geq 1$. On en déduit de nouvelles suites $\boldsymbol{a}_{1}, \boldsymbol{a}_{2}, \ldots$ et $\boldsymbol{b}_{1}, \boldsymbol{b}_{2}, \ldots$ Pour un entier $c \geq 1$, montrons que :

(2) si $a_{c}$ existe et $a_{c} \leq n_{<}$, alors $\boldsymbol{a}_{c}$ existe et on a $\boldsymbol{a}_{i}=a_{i}$ pour $i=1, \ldots, c$ et $\boldsymbol{b}_{i}=b_{i}$ pour $i=1, \ldots, c-1$;

(3) si $b_{c}$ existe et $b_{c} \leq m_{<}$, alors $\boldsymbol{b}_{c}$ existe et on a $\boldsymbol{b}_{i}=a_{i}$ et $\boldsymbol{b}_{i}=b_{i}$ pour $i=1, \ldots, c$.

On démontre ces assertions par la récurrence suivante : (2) pour $c=1$ puis (3) pour $c=1$ puis (2) pour $c=2$ puis (3) pour $c=2$ etc... Pour $c=1$, la conclusion de (2) est vérifiée : $\mathbf{a}_{1}=a_{1}=1$ par définition. Au-delà, les démonstrations de (2) et (3) étant similaires, on se contente de (2). On suppose donc que $c \geq 2$, que $a_{c}$ existe et que $a_{c} \leq n_{<}$. Puisque $\left(b_{c-1}, 1\right)<\left(a_{c}, 0\right)$, l'inégalité $a_{c} \leq n_{<}$et les définitions des entiers $n_{<}$et $m_{<}$entraînent que $b_{c-1} \leq m_{<}$. En appliquant (3) pour $c-1$, on obtient déjà que $\boldsymbol{a}_{i}=a_{i}$ et $\boldsymbol{b}_{i}=b_{i}$ pour $i=1, \ldots, c-1$. Puisque $a_{c} \leq n_{<}$, on a aussi $a_{c} \leq \boldsymbol{n}$. Puisque $a_{c} \leq n_{<}$et que les ordres $(n, m,<)$ et $(\boldsymbol{n}, \boldsymbol{m},<)$ sont équivalents, les inégalités $\left(b_{c-1}, 1\right)<(i, 0)$ et $\left(b_{c-1}, 1\right)<(i, 0)$ sont équivalentes pour $i=\left\{1, \ldots, a_{c}\right\}$. Par définition, $a_{c}$ est le plus petit élément $i \in\{1, \ldots, n\}$ tel que la première inégalité soit vérifiée. C'est donc aussi le plus petit élément de $\{1, \ldots, \mathbf{n}\}$ tel que la seconde le soit. Cette dernière définit $\boldsymbol{a}_{c}$ qui est donc égal à $a_{c}$. D'où la conclusion de (2).

En utilisant (2) et (3) et leurs symétriques en échangeant les rôles des deux ordres, on voit que les suites $a_{1}, a_{2}, \ldots$ et $\boldsymbol{a}_{1}, \boldsymbol{a}_{2}, \ldots$ contiennent les mêmes éléments de $\left\{1, \ldots, n_{<}\right\}$et que les suites $b_{1}, b_{2}, \ldots$ et $\boldsymbol{b}_{1}, \boldsymbol{b}_{2}, \ldots$ contiennent les mêmes éléments de $\left\{1, \ldots, m_{<}\right\}$. Ces suites ne diffèrent donc que par des indices $i$ pour lesquels les termes $\alpha_{i}$, resp. $\beta_{i}$, sont nuls. On voit alors que les termes $\nu_{1}$ et $\left(\alpha^{\prime}, \beta^{\prime}\right)$ construits ci-dessus ne changent pas quand on remplace l'ordre $(n, m,<)$ par $(\boldsymbol{n}, \boldsymbol{m},<)$. Les ordres obtenus sur l'ensemble d'indices de $\left(\alpha^{\prime}, \beta^{\prime}\right)$ sont équivalents d'après 1.2, Cela achève notre vérification.

Remarquons que, si $n_{<} \neq 0$, on a soit $\alpha_{1} \neq 0$, soit $\beta_{b_{1}} \neq 0$ d'après la définition de $n_{<}$. Donc $\nu_{1} \neq 0$. Il résulte de cette remarque, de la preuve ci-dessus, de (1) et de 1.2 que

(4) si $n_{<}=0, \nu_{1}=0, \alpha^{\prime}=\alpha, \beta^{\prime}=\beta$ et l'ordre $<^{\prime}$ est équivalent à $<$;

(5) si $n_{<}>0, \nu_{1} \neq 0, S\left(\alpha^{\prime}\right)+S\left(\beta^{\prime}\right)<S(\alpha)+S(\beta)$ et $n_{<^{\prime}}+m_{<^{\prime}}<n_{<}+m_{<}$.

Le procédé (b) est similaire à (a), en échangeant les rôles de $\alpha$ et $\beta$. On fixe un représentant $(n, m,<)$ de l'ordre tel que $m \geq 1$. On pose $b_{1}=1$. On note $a_{1}$ le plus petit élément de $\{1, \ldots, n\}$ tel que $(1,1)<\left(a_{1}, 0\right)$ s'il en existe, puis $b_{2}$ le plus petit élément de $\{1, \ldots, m\}$ tel que $\left(a_{1}, 0\right)<\left(b_{2}, 1\right)$ s'il en existe etc... On pose $\mu_{1}=\beta_{b_{1}}+\alpha_{a_{1}}+\beta_{b_{2}}+\alpha_{a_{2}}+\ldots$. On note $\alpha^{\prime}$ la partition extraite de $\alpha$ en supprimant les termes d'indices $a_{1}, a_{2}, \ldots$ et $\beta^{\prime}$ la partition extraite de $\beta$ en supprimant les termes d'indices $b_{1}, b_{2}, \ldots$. On munit l'ensemble d'indices de $\left(\alpha^{\prime}, \beta^{\prime}\right)$ de l'ordre induit de $(n, m,<)$.

On définit un ensemble de couples de partitions $P(\alpha, \beta,<)$ par récurrence sur $n_{<}+m_{<}$. Si $n_{<}=m_{<}=0$ c'est-à-dire si $\alpha=\beta=\emptyset$, on pose $P(\emptyset, \emptyset,<)=\{(\emptyset, \emptyset)\}$. Supposons que $n_{<}+m_{<}>0$. Si $n_{<}>0$, on applique le procédé (a), qui crée un nombre $\nu_{1}$ un couple de partitions extraites $\left(\alpha^{\prime}, \beta^{\prime}\right)$. D'après (5), l'ensemble $P\left(\alpha^{\prime}, \beta^{\prime},<^{\prime}\right)$ est déjà défini. On note $P^{a}(\alpha, \beta,<)$ l'ensemble des couples $\left(\left\{\nu_{1}\right\} \sqcup\right.$ $\left.\nu^{\prime}, \mu^{\prime}\right)$ pour $\left(\nu^{\prime}, \mu^{\prime}\right) \in P\left(\alpha^{\prime}, \beta^{\prime},<^{\prime}\right)$. Si $n_{<}=0$, on pose $P^{a}(\alpha, \beta,<)=\emptyset$. Si $m_{<}>0$, 
on applique le procédé (b), qui crée un nombre $\mu_{1}$ un couple de partitions extraites $\left(\alpha^{\prime}, \beta^{\prime}\right)$. On note $P^{b}(\alpha, \beta,<)$ l'ensemble des couples $\left(\nu^{\prime},\left\{\mu_{1}\right\} \sqcup \mu^{\prime}\right)$ pour $\left(\nu^{\prime}, \mu^{\prime}\right) \in$ $P\left(\alpha^{\prime}, \beta^{\prime},<^{\prime}\right)$. Si $m_{<}=0$, on pose $P^{b}(\alpha, \beta,<)=\emptyset$. Finalement, on pose $P(\alpha, \beta,<)$ $=P^{a}(\alpha, \beta,<) \cup P^{b}(\alpha, \beta,<)$.

Remarque. Un élément de $P(\alpha, \beta,<)$ est obtenu en appliquant une suite de procédés (a) ou (b). Cette suite n'est pas toujours unique. Par exemple, supposons $n_{<}=1$, $m_{<}=2,(1,0)<(1,1)<(2,1)$ et $\beta_{1}=\beta_{2}$. Alors le couple $\left(\left\{\alpha_{1}+\beta_{1}\right\},\left\{\beta_{2}, 0\right\}\right)$ appartient à $P(\alpha, \beta,<)$ et peut être obtenu par applications successives des procédés (a) puis (b) ou des procédés (b) puis (a).

On vérifie aisément que

(6) si $n_{<}=0$ ou $m_{<}=0, P(\alpha, \beta,<)=\{(\alpha, \beta)\}$;

(7) $S(\nu)+S(\mu)=S(\alpha)+S(\beta)$ pour tout $(\nu, \mu) \in P(\alpha, \beta,<)$.

Considérons des partitions $\alpha^{\prime}$ et $\beta^{\prime}$ extraites de $\alpha$ et $\beta$. On vérifie que

(8) soit $\left(\nu^{\prime}, \mu^{\prime}\right) \in P\left(\alpha^{\prime}, \beta^{\prime},<^{\prime}\right)$ et soit $i \in \mathbb{N}_{>0}$; alors $\nu_{i}^{\prime}$ est de la forme $\alpha_{a_{1}^{\prime}}+$ $\beta_{b_{1}^{\prime}}+\alpha_{a_{2}^{\prime}}+\ldots$, où les entiers $a_{1}^{\prime}, a_{2}^{\prime}, \ldots$, resp. $b_{1}^{\prime}, b_{2}^{\prime}, \ldots$ vérifient $\left(a_{1}^{\prime}, 0\right)<\left(b_{1}^{\prime}, 1\right)<$ $\left(a_{2}^{\prime}, 0\right)<\left(b_{2}^{\prime}, 1\right)<\ldots$

Conservons ces partitions extraites $\alpha^{\prime}$ et $\beta^{\prime}$. Rappelons que le procédé (a) crée un nombre $\nu_{1}$. Montrons que

(9) pour tout $\left(\nu^{\prime}, \mu^{\prime}\right) \in P\left(\alpha^{\prime}, \beta^{\prime},<^{\prime}\right)$, on a $\nu_{1} \geq \nu_{1}^{\prime}$.

Avec les notations de la construction (a), on a $\nu_{1}=\alpha_{a_{1}}+\beta_{b_{1}}+\alpha_{a_{2}}+\ldots$. En appliquant (8), on a aussi $\nu_{1}^{\prime}=\alpha_{a_{1}^{\prime}}+\beta_{b_{1}^{\prime}}+\alpha_{a_{2}^{\prime}}+\ldots$. Si $a_{1}^{\prime}$ existe, on a $a_{1}=1 \leq a_{1}^{\prime}$. Si $b_{1}^{\prime}$ existe, puisque $\left(a_{1}^{\prime}, 0\right)<\left(b_{1}^{\prime}, 1\right)$, on a aussi $\left(a_{1}, 0\right)<\left(b_{1}^{\prime}, 1\right)$. Puisque $b_{1}$ est le plus petit indice vérifiant cette inégalité, $b_{1}$ existe et $b_{1} \leq b_{1}^{\prime}$. Le procédé se poursuit et on obtient que si un élément des suites $a_{1}^{\prime}, a_{2}^{\prime}, \ldots$ ou $b_{1}^{\prime}, b_{2}^{\prime}, \ldots$ existe, l'élément correspondant des suites $a_{1}, a_{2}, \ldots$ ou $b_{1}, b_{2}, \ldots$ existe aussi et lui est inférieur ou égal. L’inégalité cherchée en résulte.

Il résulte de (9) que, pour tout élément $(\nu, \mu) \in P^{a}(\alpha, \beta,<), \nu_{1}$ est bien le plus grand élément de $\nu$ (autrement dit, la notation est cohérente).

Le procédé (a) crée des suites $a_{1}=1, a_{2}, \ldots, b_{1}, b_{2}, \ldots$, un élément $\nu_{1}=\alpha_{a_{1}}+\beta_{b_{1}}+$ $\alpha_{a_{2}}+\ldots$ et des partitions $\alpha^{\prime}, \beta^{\prime}$. Le procédé (b) crée des objets qu'ici, nous notons en les soulignant : des suites $\underline{b}_{1}=1, \underline{b}_{2}, \ldots, \underline{a}_{1}, \underline{a}_{2}, \ldots$, un élément $\underline{\mu}_{1}=\beta_{\underline{b}_{1}}+\alpha_{\underline{a}_{1}}+\beta_{\underline{b}_{2}}+\ldots$ et des partitions $\underline{\alpha}^{\prime}, \beta^{\prime}$. Supposons $(1,0)<(1,1)$. On voit que $b_{1}=1$, puis, par récurrence, $\underline{a}_{1}=a_{2}, \underline{b}_{2}=b_{2}, \underline{a}_{2}=a_{3} \ldots$ D'où $\nu_{1}=\alpha_{1}+\underline{\mu}_{1}, \underline{\alpha}^{\prime}=\left\{\alpha_{1}\right\} \sqcup \alpha^{\prime}, \underline{\beta}^{\prime}=\beta^{\prime}$. Il y a un résultat similaire si $(1,1)<(1,0)$. En résumé :

(10) si $(1,0)<(1,1), \nu_{1}=\alpha_{1}+\underline{\mu}_{1}, \underline{\alpha}^{\prime}=\left\{\alpha_{1}\right\} \sqcup \alpha^{\prime}, \underline{\beta}^{\prime}=\beta^{\prime}$; si $(1,1)<(1,0)$, $\underline{\mu}_{1}=\beta_{1}+\nu_{1}, \alpha^{\prime}=\underline{\alpha}^{\prime}, \beta^{\prime}=\left\{\beta_{1}\right\} \sqcup \underline{\beta}^{\prime}$.

1.4. L'ensemble $P_{A, B ; s}(\alpha, \beta,<)$. Soient deux réels $A, B$ et un réel $s>0$. On introduit les éléments suivants de $\mathcal{R}$ :

$$
\left[A,-\infty\left[s=\{A, A-s, A-2 s, \ldots\},\left[B,-\infty\left[{ }_{s}=\{B, B-s, B-2 s, \ldots\}\right.\right.\right.\right.
$$

et, pour $\nu, \mu \in \mathcal{P}$,

$$
\Lambda_{A, B ; s}(\nu, \mu)=(\nu+[A,-\infty[s) \sqcup(\mu+[B,-\infty[s) .
$$

Appliquons à $(\alpha, \beta)$ le procédé (a) du paragraphe précédent, qui crée un nombre $\nu_{1}$ et des partitions extraites $\left(\alpha^{\prime}, \beta^{\prime}\right)$. Soit $\left(\nu^{\prime}, \mu^{\prime}\right) \in P\left(\alpha^{\prime}, \beta^{\prime},<^{\prime}\right)$, posons $\nu=\left\{\nu_{1}\right\} \sqcup \nu^{\prime}$, $\mu=\mu^{\prime}$. Alors, d'après 1.3 $(9)$, on a

(1) $\Lambda_{A, B ; s}(\nu, \mu)=\left\{\nu_{1}+A\right\} \sqcup \Lambda_{A-s, B ; s}\left(\nu^{\prime}, \mu^{\prime}\right)$. 
On définit un sous-ensemble $\left.P_{A, B ; s}(\alpha, \beta),<\right)$ de $P(\alpha, \beta,<)$ par récurrence sur $n_{<}+m_{<}$. Si $\alpha=\beta=\emptyset, P_{A, B ; s}(\emptyset, \emptyset,<)=\{(\emptyset, \emptyset)\}$. Supposons $n_{<}+m_{<}>0$. Considérons les deux conditions

(2) $n_{<}>0$;

(3) $m_{<}=0$, ou $(1,0)<(1,1)$ et $\alpha_{1}+A \geq B$, ou $(1,1)<(1,0)$ et $\beta_{1}+B \leq A$.

Si elles sont toutes deux satisfaites, on utilise le procédé (a) qui crée un élément $\nu_{1}$ et des partitions $\alpha^{\prime}$ et $\beta^{\prime}$ et on note $P_{A, B ; s}^{a}(\alpha, \beta ;<)$ l'ensemble des couples $\left(\left\{\nu_{1}\right\} \sqcup\right.$ $\left.\nu^{\prime}, \mu^{\prime}\right)$ pour $\left(\nu^{\prime}, \mu^{\prime}\right) \in P_{A-s, B ; s}\left(\alpha^{\prime}, \beta^{\prime},<^{\prime}\right)$. Si (2) ou (3) n'est pas vérifiée, on pose $P_{A, B ; s}^{a}(\alpha, \beta ;<)=\emptyset$.

Considérons les conditions

(4) $m_{<}>0$;

(5) $n_{<}=0$, ou $(1,0)<(1,1)$ et $\alpha_{1}+A \leq B$, ou $(1,1)<(1,0)$ et $\beta_{1}+B \geq A$.

Si elles sont toutes deux satisfaites, on utilise le procédé (b) qui crée un élément $\mu_{1}$ et des partitions $\alpha^{\prime}$ et $\beta^{\prime}$ et on note $P_{A, B ; s}^{b}(\alpha, \beta ;<)$ l'ensemble des couples $\left(\nu^{\prime},\left\{\mu_{1}\right\} \sqcup \mu^{\prime}\right)$ pour $\left(\nu^{\prime}, \mu^{\prime}\right) \in P_{A, B-s ; s}\left(\alpha^{\prime}, \beta^{\prime},<^{\prime}\right)$. Si (4) ou (5) n'est pas vérifiée, on pose $P_{A, B ; s}^{b}(\alpha, \beta ;<)=\emptyset$.

Finalement, on pose $\left.P_{A, B ; s}(\alpha, \beta,<)=P_{A, B ; s}^{a}(\alpha, \beta),<\right) \cup P_{A, B ; s}^{b}(\alpha, \beta,<)$. On voit que les conditions d'au moins un des couples (2) et (3) ou (4) et (5) sont satisfaites, donc $P_{A, B ; s}(\alpha, \beta,<)$ est non vide. Il peut contenir plusieurs éléments puisque les conditions des deux couples peuvent être vérifiées. Mais reprenons la construction en remplaçant la condition (3) par la condition plus forte

(3') $m_{<}=0$, ou $(1,0)<(1,1)$ et $\alpha_{1}+A>B$, ou $(1,1)<(1,0)$ et $\beta_{1}+B<A$.

Alors les conditions d'un unique couple (2) et (3') ou (4) et (5) sont vérifiées. La construction fournit alors un unique élément de $P_{A, B ; s}(\alpha, \beta,<)$ que nous appelons l'élément canonique de type (b). On définit de même un élément canonique de type (a) en renforçant la condition (5).

Supposons $n_{<}>0$ et $m_{<}>0$. Soit $(\nu, \mu) \in P_{A, B ; s}(\alpha, \beta,<)$. Si $(\nu, \mu) \in P_{A, B ; s}^{a}(\alpha$, $\beta,<)$, posons $\lambda_{1}=\nu_{1}+A$. Si $(\nu, \mu) \in P_{A, B ; s}^{b}(\alpha, \beta,<)$, posons $\lambda_{1}=\mu_{1}+B$. Alors

(6) $\lambda_{1}$ est le plus grand terme de $\Lambda_{A, B ; s}(\nu, \mu)$.

On ne perd rien à supposer que $(\nu, \mu) \in P_{A, B ; s}^{a}(\alpha, \beta,<)$. On applique le procédé (a) qui crée le nombre $\nu_{1}$ et des partitions extraites $\left(\alpha^{\prime}, \beta^{\prime}\right)$. Soit $\left(\nu^{\prime}, \mu^{\prime}\right) \in$ $P_{A-s, B ; s}\left(\alpha^{\prime}, \beta^{\prime},<^{\prime}\right)$ tel que $\nu=\left\{\nu_{1}\right\} \sqcup \nu^{\prime}, \mu=\mu^{\prime}$. Notons $\lambda_{2}$ le plus grand terme de $\Lambda_{A-s, B ; s}\left(\nu^{\prime}, \mu^{\prime}\right)$. D'après (1), on doit prouver que $\lambda_{1} \geq \lambda_{2}$. On a $\lambda_{2}=\sup \left(\nu_{1}^{\prime}+\right.$ $\left.A-s, \mu_{1}^{\prime}+B\right)$. D'après [1.3 (9), on a $\nu_{1}^{\prime} \leq \nu_{1}$, donc $\nu_{1}^{\prime}+A-s<\nu_{1}+A=\lambda_{1}$. Notons $\underline{\mu}_{1}$ le nombre créé par le procédé (b). D'après l'assertion symétrique de $1.3(9)$, on a $\mu_{1}^{\prime} \leq \underline{\mu}_{1}$. Supposons $(1,0)<(1,1)$. Puisque $P_{A, B ; s}^{a}(\alpha, \beta,<)$ n'est pas vide, on a $\alpha_{1}+A \geq B$. D'après [1.3(10), on a $\nu_{1}=\alpha_{1}+\underline{\mu}_{1}$. Donc $\mu_{1}^{\prime}+B \leq$ $\mu_{1}+B=\nu_{1}-\alpha_{1}+B \leq \nu_{1}+A=\lambda_{1}$. Supposons maintenant $(1,1)<(1,0)$. Puisque $P_{A, B ; s}^{a}(\alpha, \beta,<)$ n'est pas vide, on a $\beta_{1}+B \leq A$. D'après 1.3(10), on a $\underline{\mu}_{1}=\beta_{1}+\nu_{1}$. Donc $\mu_{1}^{\prime}+B \leq \underline{\mu}_{1}+B=\beta_{1}+B+\nu_{1} \leq A+\nu_{1}=\lambda_{1}$. Cela démontre (6).

Supposons toujours $n_{<}>0$ et $m_{<}>0$, soit $(\nu, \mu)$ l'élément canonique de type (b). On a alors l'inégalité plus précise suivante :

(7) supposons que $(\nu, \mu) \in P_{A, B ; s}^{a}(\alpha, \beta,<)$; alors $\lambda_{1}$ est strictement plus grand que tous les autres termes de $\Lambda_{A, B ; s}(\nu, \mu)$.

Par définition de l'élément canonique de type (b), puisque $(\nu, \mu) \in P_{A, B ; s}^{a}(\alpha, \beta,<)$, c'est que $P_{A, B ; s}^{b}(\alpha, \beta,<)$ est vide. On a donc les inégalités strictes $\alpha_{1}+A>B$ si 
$(1,0)<(1,1)$ et $\beta_{1}+B<A$ si $(1,1)<(1,0)$. En glissant ces inégalités strictes dans la preuve ci-dessus, on obtient $(7)$.

La construction de l'ensemble $P_{A, B ; s}(\alpha, \beta,<)$ ne dépend évidemment que de $A-B$. Autrement dit

(8) pour tout réel $C$, on a l'égalité $P_{A+C, B+C ; s}(\alpha, \beta,<)=P_{A, B ; s}(\alpha, \beta)$.

1.5. La partition $\alpha^{-}$. Pour $\lambda=\left(\lambda_{1}, \lambda_{2}, \ldots\right) \in \mathcal{P}$, on note $\lambda^{-}=\left(\lambda_{2}, \lambda_{3}, \ldots\right)$ la partition extraite obtenue en supprimant $\lambda_{1}$. Le couple $\left(\alpha^{-}, \beta\right)$ est un couple de partitions extraites de $(\alpha, \beta)$ et on munit son ensemble d'indices de l'ordre induit, que l'on note $<^{-}$. On vérifie aisément les propriétés suivantes :

(1) si $(1,0)<(1,1)$, on a l'égalité $m_{<}=m_{<^{\prime}}$;

(2) si $(1,0)<(1,1)$ et $n_{<^{\prime}}=0$, la situation est la suivante : $\alpha=\left(\alpha_{1}, 0 \ldots\right)$, $\beta=\left(\beta_{1}, \ldots, \beta_{m}, 0 \ldots\right)$, avec $(1,0)<(1,1)<\ldots<(m, 1)<(2,0)$; pour $i \geq 1$, posons $\nu^{i}=\left(\alpha_{1}+\beta_{i}, 0, \ldots\right), \mu^{i}=\left(\beta_{1}, \ldots, \beta_{i-1}, \beta_{i+1}, \ldots\right) ;$ alors $P(\alpha, \beta,<)=\left\{\left(\nu^{i}, \mu^{i}\right) ; i \in\right.$ $\left.\mathbb{N}_{>0}\right\}$.

Montrons que

(3) si $m_{<^{\prime}}=0$ et $\left(\alpha^{-}, \beta\right) \neq(\emptyset, \emptyset)$, alors $m_{<}=0$.

En effet, l'hypothèse $m_{<^{\prime}}=0$ implique que $\beta=\emptyset$. La seconde hypothèse implique alors $\alpha^{-} \neq \emptyset$. Par définition de $m_{<^{\prime}}$, on a $\left(t\left(\alpha^{-}\right), 0\right)<^{\prime}(1,1)$. Alors $(t(\alpha), 0)<(1,1)$, d'où $m_{<}=0$.

Les propriétés suivantes se démontrent comme 1.3(10). Appliquons à $(\alpha, \beta)$ le procédé $(\mathrm{a})$. Il construit un nombre $\nu_{1}$ et des partitions $\left(\alpha^{\prime}, \beta^{\prime}\right)$. Alors

(4) si $(1,0)<(1,1)$, le procédé (b) appliqué à $\left(\alpha^{-}, \beta\right)$ construit le nombre $\underline{\mu}_{1}=$ $\nu_{1}-\alpha_{1}$ et les mêmes partitions $\left(\alpha^{\prime}, \beta^{\prime}\right)$;

(5) si $(1,0)<^{-}(1,1)$, ce qui équivaut à $(2,0)<(1,1)$, le procédé $(\mathrm{a})$ appliqué à $\left(\alpha^{-}, \beta\right)$ construit le nombre $\underline{\nu}_{1}=\nu_{1}-\alpha_{1}+\alpha_{2}$ et les partitions $\left(\alpha^{\prime-}, \beta^{\prime}\right)$;

(6) si $(1,0)<(1,1)$ et $(1,1)<^{-}(1,0)$, ce qui équivaut à $(1,1)<(2,0)$, le procédé (a) appliqué à $\left(\alpha^{-}, \beta\right)$ construit le nombre $\underline{\nu}_{1}=\nu_{1}-\alpha_{1}-\beta_{1}$ et les partitions $\left(\alpha^{\prime},\left\{\beta_{1}\right\} \sqcup \beta^{\prime}\right)$.

Appliquons à $(\alpha, \beta)$ le procédé (b). Il construit un nombre $\mu_{1}$ et des partitions, notons-les $\left(\alpha^{\prime \prime}, \beta^{\prime \prime}\right)$. Alors

(7) si $(1,0)<(1,1)$, le procédé $(\mathrm{b})$ appliqué à $\left(\alpha^{-}, \beta\right)$ construit le même nombre $\mu_{1}$ et les partitions $\left(\alpha^{\prime \prime}, \beta^{\prime \prime}\right)$.

Supposons que l'ensemble $P_{A, B ; s}^{b}(\alpha, \beta ;<)$ n'est pas vide. Soient $A^{\prime}, B^{\prime}$ des réels tels que $A \geq A^{\prime}, B^{\prime} \geq B$. Montrons que

(8) si $\left(\alpha^{-}, \beta\right) \neq(\emptyset, \emptyset)$, l'ensemble $P_{A^{\prime}, B^{\prime} ; s}^{b}\left(\alpha^{-}, \beta,<^{-}\right)$n'est pas vide.

Puisque $P_{A, B ; s}^{b}(\alpha, \beta ;<)$ n'est pas vide, on a $m_{<}>0$. D'après $(3)$, on a aussi $m_{<^{\prime}}>0$. Si $n_{<^{-}}=0$, l'analogue de 1.4(5) est vérifiée, donc $P_{A^{\prime}, B^{\prime} ; s}^{b}\left(\alpha^{-}, \beta,<^{-}\right)$ n'est pas vide. Supposons $n_{<^{-}} \neq 0$, donc aussi $n_{<} \neq 0$. Supposons d'abord $(1,0)<^{-}$ $(1,1)$, c'est-à-dire $(2,0)<(1,1)$ donc aussi $(1,0)<(1,1)$. Pour vérifier l'analogue de la condition 1.4 $(5)$, on doit montrer que $\alpha_{1}^{-}+A^{\prime} \leq B^{\prime}$. Cette condition pour $(\alpha, \beta)$ nous dit que $\alpha_{1}+A \leq B$. Or

$$
\alpha_{1}^{-}+A^{\prime}=\alpha_{2}+A^{\prime} \leq \alpha_{1}+A \leq B \leq B^{\prime} .
$$

Supposons maintenant $(1,1)<^{-}(1,0)$, mais $(1,0)<(1,1)$. On a toujours $\alpha_{1}+A \leq$ $B$ et on doit montrer que $\beta_{1}+B^{\prime} \geq A^{\prime}$. Mais

$$
\beta_{1}+B^{\prime} \geq B^{\prime} \geq B \geq \alpha_{1}+A \geq A \geq A^{\prime} .
$$


Supposons enfin $(1,1)<(1,0)$, a fortiori $(1,1)<^{-}(1,0)$. On a cette fois $\beta_{1}+B \geq A$ et on doit montrer que $\beta_{1}+B^{\prime} \geq A^{\prime}$. Mais

$$
\beta_{1}+B^{\prime} \geq \beta_{1}+B \geq A \geq A^{\prime} .
$$

Cela prouve (8).

1.6. Définition de $p_{A, B ; s}(\alpha, \beta,<)$.

Lemme. Soient $(\nu, \mu) \in P(\alpha, \beta,<)$ et $(\boldsymbol{\nu}, \boldsymbol{\mu}) \in P_{A, B ; s}(\alpha, \beta,<)$. Alors

(i) $\Lambda_{A, B ; s}(\nu, \mu) \leq \Lambda_{A, B ; s}(\boldsymbol{\nu}, \boldsymbol{\mu})$;

(ii) on a $\Lambda_{A, B ; s}(\nu, \mu)=\Lambda_{A, B ; s}(\boldsymbol{\nu}, \boldsymbol{\mu})$ si et seulement si $(\nu, \mu) \in P_{A, B ; s}(\alpha, \beta,<)$.

Preuve. L'assertion "si" de (ii) résulte trivialement de (i). On va donc démontrer cette assertion (i) ainsi que l'assertion "seulement si" de (ii).

On raisonne par récurrence sur $n_{<}+m_{<}$. Si ce nombre est nul, ou plus généralement si $n_{<}=0$ ou $m_{<}=0, P_{A, B ; s}(\alpha, \beta,<)=P(\alpha, \beta,<)=\{(\alpha, \beta)\}$ et les assertions sont triviales. Supposons $n_{<} \geq 1$ et $m_{<} \geq 1$. Les éléments $(\nu, \mu)$ et $(\boldsymbol{\nu}, \boldsymbol{\mu})$ sont construits selon des procédés (a) ou (b). Supposons d'abord qu'ils soient construits par le même procédé, disons par le procédé (a). Celui-ci construit un terme $\nu_{1}$ et un couple de partitions $\left(\alpha^{\prime}, \beta^{\prime}\right)$. On a $\nu=\left\{\nu_{1}\right\} \sqcup \nu^{\prime}$ et $\mu=\mu^{\prime}$ pour un élément $\left(\nu^{\prime}, \mu^{\prime}\right) \in P\left(\alpha^{\prime}, \beta^{\prime},<^{\prime}\right)$. On en déduit l'égalité

$$
\Lambda_{A, B ; s}(\nu, \mu)=\left\{\nu_{1}+A\right\} \sqcup \Lambda_{A-s, B ; s}\left(\nu^{\prime}, \mu^{\prime}\right) .
$$

De même

$$
\Lambda_{A, B ; s}(\boldsymbol{\nu}, \boldsymbol{\mu})=\left\{\nu_{1}+A\right\} \sqcup \Lambda_{A-s, B ; s}\left(\boldsymbol{\nu}^{\prime}, \boldsymbol{\mu}^{\prime}\right),
$$

où $\left(\boldsymbol{\nu}^{\prime}, \boldsymbol{\mu}^{\prime}\right)$ est un élément de $P_{A-s, B ; s}\left(\alpha^{\prime}, \beta^{\prime},<^{\prime}\right)$. Alors l'inégalité $\Lambda_{A, B ; s}(\nu, \mu) \leq$ $\Lambda_{A, B ; s}(\boldsymbol{\nu}, \boldsymbol{\mu})$ équivaut d'après le lemme 1.1 à l'inégalité $\Lambda_{A-s, B ; s}\left(\nu^{\prime}, \mu^{\prime}\right) \leq$ $\Lambda_{A-s, B ; s}\left(\boldsymbol{\nu}^{\prime}, \boldsymbol{\mu}^{\prime}\right)$ qui est connue par récurrence. La première inégalité est une égalité si et seulement s'il en est de même pour la seconde. Pour celle-ci, l'égalité entraîne par récurrence que $\left(\nu^{\prime}, \mu^{\prime}\right) \in P_{A-s, B ; s}\left(\alpha^{\prime}, \beta^{\prime},<^{\prime}\right)$. Mais alors, par construction, on a $(\nu, \mu) \in P_{A, B ; s}(\alpha, \beta)$.

Supposons maintenant que $(\nu, \mu)$ et $(\boldsymbol{\nu}, \boldsymbol{\mu})$ soient construits selon des procédés différents. On ne perd rien à supposer $(1,0)<(1,1)$. Supposons d'abord que $(\boldsymbol{\nu}, \boldsymbol{\mu})$ soit construit à l'aide du procédé (a) et que $(\nu, \mu)$ le soit selon le procédé (b). Le procédé (a) définit un élément que l'on note ici $\boldsymbol{\nu}_{1}$ et un couple de partitions extraites $\left(\boldsymbol{\alpha}^{\prime}, \boldsymbol{\beta}^{\prime}\right)$. Le procédé (b) définit un terme $\mu_{1}$ et un couple de partitions extraites $\left(\alpha^{\prime}, \beta^{\prime}\right)$. D'après $1.3(10)$, l'hypothèse $(1,0)<(1,1)$ entraîne que $\boldsymbol{\nu}_{1}=$ $\alpha_{1}+\mu_{1}, \alpha^{\prime}=\left\{\alpha_{1}\right\} \sqcup \boldsymbol{\alpha}^{\prime}, \beta^{\prime}=\boldsymbol{\beta}^{\prime}$. Plus précisément, l'indice (1,0) se conserve dans la construction de $\left(\alpha^{\prime}, \beta^{\prime}\right)$ (on n'enlève pas le premier indice de la partition $\alpha$ pour construire la partition extraite $\left.\alpha^{\prime}\right)$. Puisque $(1,0)$ est le plus petit indice pour l'ordre $<$, c'est aussi le plus petit pour l'ordre $<^{\prime}$. Les relations ci-dessus entraînent

$$
\begin{gathered}
\Lambda_{A, B ; s}(\boldsymbol{\nu}, \boldsymbol{\mu})=\left\{\alpha_{1}+\mu_{1}+A\right\} \sqcup \Lambda_{A-s, B ; s}\left(\nu^{\prime}, \boldsymbol{\mu}^{\prime}\right), \\
\Lambda_{A, B ; s}(\nu, \mu)=\left\{\mu_{1}+B\right\} \sqcup \Lambda_{A, B-s ; s}\left(\nu^{\prime}, \mu^{\prime}\right),
\end{gathered}
$$

pour des couples $\left(\boldsymbol{\nu}^{\prime}, \boldsymbol{\mu}^{\prime}\right) \in P_{A-s, B ; s}\left(\boldsymbol{\alpha}^{\prime}, \boldsymbol{\beta}^{\prime},<^{\prime}\right)$ et $\left(\nu^{\prime}, \mu^{\prime}\right) \in P\left(\alpha^{\prime}, \beta^{\prime},<^{\prime}\right)$.

Introduisons un couple $\left(\underline{\nu}^{\prime}, \underline{\mu}^{\prime}\right) \in P_{A, B-s ; s}\left(\alpha^{\prime}, \beta^{\prime},<^{\prime}\right)$. Puisque $P_{A, B ; s}^{a}(\alpha, \beta,<)$ contient $(\boldsymbol{\nu}, \boldsymbol{\mu})$ et est donc non vide, la relation 1.4(3) est vérifiée et $\alpha_{1}+A \geq$ $B$. A fortiori $\alpha_{1}+A>B-s$. Supposons $n_{<^{\prime}}>0$. Alors $\left(\underline{\nu}^{\prime}, \mu^{\prime}\right)$ appartient à $P_{A, B-s ; s}^{a}\left(\alpha^{\prime}, \beta^{\prime},<^{\prime}\right)$. Le procédé (a) appliqué à $\left(\alpha^{\prime}, \beta^{\prime}\right)$ construit un nombre $\underline{\nu}_{1}^{\prime}$ et 
un couple de partitions extraites $\left(\alpha^{\prime \prime}, \beta^{\prime \prime}\right)$. On a $\left(\underline{\nu}^{\prime}, \underline{\mu}^{\prime}\right)=\left(\underline{\nu}_{1}^{\prime} \sqcup \nu^{\prime \prime}, \mu^{\prime \prime}\right)$, pour un couple $\left(\nu^{\prime \prime}, \mu^{\prime \prime}\right) \in P_{A-s, B-s ; s}\left(\alpha^{\prime \prime}, \beta^{\prime \prime},<^{\prime \prime}\right)$. On a

$$
\Lambda_{A, B-s ; s}\left(\underline{\nu}^{\prime}, \underline{\mu}^{\prime}\right)=\left\{\underline{\nu}_{1}^{\prime}+A\right\} \sqcup \Lambda_{A-s, B-s ; s}\left(\nu^{\prime \prime}, \mu^{\prime \prime}\right) .
$$

Levons l'hypothèse $n_{<^{\prime}}>0$. Si $n_{<^{\prime}}=0$, on a $\alpha^{\prime}=\beta^{\prime}=\emptyset$ puisque $(1,0)$ est le plus petit élément de l'ensemble d'indices de $\left(\alpha^{\prime}, \beta^{\prime}\right)$. Alors $\underline{\nu}_{1}^{\prime}=0$ et l'égalité ci-dessus est encore vérifiée, avec $\left(\nu^{\prime \prime}, \mu^{\prime \prime}\right)=(\emptyset, \emptyset)$. L'hypothèse de récurrence entraîne que $\Lambda_{A, B-s ; s}\left(\nu^{\prime}, \mu^{\prime}\right) \leq \Lambda_{A, B-s ; s}\left(\underline{\nu}^{\prime}, \underline{\mu}^{\prime}\right)$. Avec (2), on obtient

$$
\Lambda_{A, B ; s}(\nu, \mu) \leq\left\{\mu_{1}+B\right\} \sqcup\left\{\underline{\nu}_{1}^{\prime}+A\right\} \sqcup \Lambda_{A-s, B-s ; s}\left(\nu^{\prime \prime}, \mu^{\prime \prime}\right) .
$$

Notons $\bar{\mu}_{1}^{\prime}$ le nombre issu du procédé (b) appliqué à $\left(\alpha^{\prime}, \beta^{\prime}\right)$. Puisque $(1,0)<^{\prime}(1,1)$, on a $\underline{\nu}_{1}^{\prime}=\alpha_{1}^{\prime}+\bar{\mu}_{1}^{\prime}=\alpha_{1}+\bar{\mu}_{1}^{\prime}$ d'après 1.3 (10). D'après l'assertion symétrique de 1.3 (3), on a $\mu_{1} \geq \bar{\mu}_{1}^{\prime}$. En utilisant l'inégalité $\alpha_{1}+A \geq B$, on voit que $\left\{\mu_{1}+B\right\} \sqcup\left\{\underline{\nu}_{1}^{\prime}+A\right\} \leq$ $\left\{\alpha_{1}+\mu_{1}+A\right\} \sqcup\left\{\bar{\mu}_{1}^{\prime}+B\right\}$. Donc

$$
\Lambda_{A, B ; s}(\nu, \mu) \leq\left\{\alpha_{1}+\mu_{1}+A\right\} \sqcup\left\{\bar{\mu}_{1}^{\prime}+B\right\} \sqcup \Lambda_{A-s, B-s ; s}\left(\nu^{\prime \prime}, \mu^{\prime \prime}\right) .
$$

D'après 1.5(4) et 1.5(7), le procédé (b) appliqué à $\left(\boldsymbol{\alpha}^{\prime}, \boldsymbol{\beta}^{\prime}\right)$ crée le nombre $\bar{\mu}_{1}^{\prime}$ et le couple de partions $\left(\alpha^{\prime \prime}, \beta^{\prime \prime}\right)$. Donc $\left(\nu^{\prime \prime},\left\{\bar{\mu}_{1}^{\prime}\right\} \sqcup \mu^{\prime \prime}\right)$ est un élément de $P\left(\boldsymbol{\alpha}^{\prime}, \boldsymbol{\beta}^{\prime},<^{\prime}\right)$ et on a

$$
\Lambda_{A-s, B ; s}\left(\nu^{\prime \prime},\left\{\bar{\mu}_{1}^{\prime}\right\} \sqcup \mu^{\prime \prime}\right)=\left\{\bar{\mu}_{1}^{\prime}+B\right\} \sqcup \Lambda_{A-s, B-s ; s}\left(\nu^{\prime \prime}, \mu^{\prime \prime}\right) .
$$

Par récurrence, on a

$$
\Lambda_{A-s, B ; s}\left(\nu^{\prime \prime},\left\{\bar{\mu}_{1}^{\prime}\right\} \sqcup \mu^{\prime \prime}\right) \leq \Lambda_{A-s, B ; s}\left(\nu^{\prime}, \boldsymbol{\mu}^{\prime}\right)
$$

et, avec (3), on obtient

$$
\Lambda_{A, B ; s}(\nu, \mu) \leq\left\{\alpha_{1}+\mu_{1}+A\right\} \sqcup \Lambda_{A-s, B ; s}\left(\nu^{\prime}, \boldsymbol{\mu}^{\prime}\right) .
$$

En comparant avec (1), on obtient l'inégalité cherchée $\Lambda_{A, B ; s}(\nu, \mu) \leq \Lambda_{A, B ; s}(\boldsymbol{\nu}, \boldsymbol{\mu})$.

Supposons que $\Lambda_{A, B ; s}(\nu, \mu)=\Lambda_{A, B ; s}(\boldsymbol{\nu}, \boldsymbol{\mu})$. Alors toutes les inégalités ci-dessus doivent être des égalités. D'abord $\Lambda_{A, B-s ; s}\left(\nu^{\prime}, \mu^{\prime}\right)=\Lambda_{A, B-s ; s}\left(\underline{\nu}^{\prime}, \mu^{\prime}\right)$. Par récurrence, cela entraîne que $\left(\nu^{\prime}, \mu^{\prime}\right) \in P_{A, B-s ; s}\left(\alpha^{\prime}, \beta^{\prime}\right)$. Puisque le couple $\left(\underline{\nu}^{\prime}, \mu^{\prime}\right)$ était un élément quelconque de cet ensemble, on peut supposer qu'il est égal à $\left(\nu^{\prime}, \mu^{\prime}\right)$. Ensuite $\left\{\mu_{1}+B\right\} \sqcup\left\{\underline{\nu}_{1}^{\prime}+A\right\}=\left\{\alpha_{1}+\mu_{1}+A\right\} \sqcup\left\{\bar{\mu}_{1}^{\prime}+B\right\}$. Cela entraîne que $\alpha_{1}+A=B$ ou que $\bar{\mu}_{1}^{\prime}=\mu_{1}$. Dans le premier cas, le procédé (b) est permis pour définir l'ensemble $P_{A, B ; s}^{b}(\alpha, \beta,<)$. Le couple $(\nu, \mu)$ est construit à l'aide de ce procédé et de l'élément $\left(\nu^{\prime}, \mu^{\prime}\right) \in P_{A, B-s ; s}\left(\alpha^{\prime}, \beta^{\prime},<^{\prime}\right)$. Donc $(\nu, \mu) \in P_{A, B ; s}(\alpha, \beta,<)$. Supposons maintenant $\bar{\mu}_{1}^{\prime}=\mu_{1}$. On doit aussi avoir l'égalité $\Lambda_{A-s, B ; s}\left(\nu^{\prime \prime},\left\{\bar{\mu}_{1}^{\prime}\right\} \sqcup \mu^{\prime \prime}\right)=\Lambda_{A-s, B ; s}\left(\boldsymbol{\nu}^{\prime}, \boldsymbol{\mu}^{\prime}\right)$, ce qui entraîne par récurrence que $\left(\nu^{\prime \prime},\left\{\bar{\mu}_{1}^{\prime}\right\} \sqcup \mu^{\prime \prime}\right) \in P_{A-s, B ; s}\left(\boldsymbol{\alpha}^{\prime}, \boldsymbol{\beta}^{\prime},<^{\prime}\right)$. L'élément de $P_{A, B ; s}^{a}(\alpha, \beta,<)$ construit à l'aide de ce couple est $\left(\left\{\boldsymbol{\nu}_{1}\right\} \sqcup \nu^{\prime \prime},\left\{\bar{\mu}_{1}^{\prime}\right\} \sqcup \mu^{\prime \prime}\right)$, ou encore $\left(\left\{\alpha_{1}+\mu_{1}\right\} \sqcup \nu^{\prime \prime},\left\{\bar{\mu}_{1}^{\prime}\right\} \sqcup \mu^{\prime \prime}\right)$. Le couple $(\nu, \mu)$ est

$\left(\nu^{\prime},\left\{\mu_{1}\right\} \sqcup \mu^{\prime}\right)=\left(\underline{\nu}^{\prime},\left\{\mu_{1}\right\} \sqcup \underline{\mu}^{\prime}\right)=\left(\left\{\underline{\nu}_{1}^{\prime}\right\} \sqcup \nu^{\prime \prime},\left\{\mu_{1}\right\} \sqcup \mu^{\prime \prime}\right)=\left(\left\{\alpha_{1}+\bar{\mu}_{1}^{\prime}\right\} \sqcup \nu^{\prime \prime},\left\{\mu_{1}\right\} \sqcup \mu^{\prime \prime}\right)$.

Puisque $\bar{\mu}_{1}^{\prime}=\mu_{1}$, ce couple est égal au précédent, donc $(\nu, \mu)$ appartient à $P_{A, B ; s}(\alpha, \beta)$.

On suppose maintenant que $(\nu, \mu)$ est construit à l'aide du procédé (a) et que $(\boldsymbol{\nu}, \boldsymbol{\mu})$ l'est à l'aide du procédé (b) (on suppose toujours $(1,0)<(1,1)$ ). Cette dernière condition impose que la relation 1.4(3) est vérifiée, donc $\alpha_{1}+A \leq B$.On reprend le raisonnement en échangeant les rôles de $(\nu, \mu)$ et $(\boldsymbol{\nu}, \boldsymbol{\mu})$. Les égalités (1) et (2) sont inversées, c'est-à-dire que l'on a

$$
\begin{gathered}
\Lambda_{A, B ; s}(\boldsymbol{\nu}, \boldsymbol{\mu})=\left\{\boldsymbol{\mu}_{1}+B\right\} \sqcup \Lambda_{A, B-s ; s}\left(\boldsymbol{\nu}^{\prime}, \boldsymbol{\mu}^{\prime}\right), \\
\Lambda_{A, B ; s}(\nu, \mu)=\left\{\alpha_{1}+\boldsymbol{\mu}_{1}+A\right\} \sqcup \Lambda_{A-s, B ; s}\left(\nu^{\prime}, \mu^{\prime}\right),
\end{gathered}
$$


pour des couples $\left(\boldsymbol{\nu}^{\prime}, \boldsymbol{\mu}^{\prime}\right) \in P_{A, B-s ; s}\left(\boldsymbol{\alpha}^{\prime}, \boldsymbol{\beta}^{\prime},<^{\prime}\right)$ et $\left(\nu^{\prime}, \mu^{\prime}\right) \in P\left(\alpha^{\prime}, \beta^{\prime}\right)$. Cette fois, $\boldsymbol{\alpha}^{\prime}=\left\{\alpha_{1}\right\} \sqcup \alpha^{\prime}$ tandis que $\boldsymbol{\beta}^{\prime}=\beta^{\prime}$.

Introduisons un couple $\left(\underline{\nu}^{\prime}, \underline{\mu}^{\prime}\right) \in P_{A-s, B ; s}\left(\alpha^{\prime}, \beta^{\prime},<^{\prime}\right)$. Supposons provisoirement que $m_{>^{\prime}} \geq 1$ et montrons que le couple $\left(\underline{\nu}^{\prime}, \underline{\mu}^{\prime}\right)$ appartient à $P_{A-s, B ; s}^{b}\left(\alpha^{\prime}, \beta^{\prime},<^{\prime}\right)$. C'est évident si $n_{<^{\prime}}=0$ puisqu'alors seul le procédé (b) est autorisé. Supposons $n_{<^{\prime}} \geq 1$. Contrairement à ce qui se passait plus haut, l'élément $(1,0)$ de l'ensemble d'indices de $(\alpha, \beta)$ ne s'identifie plus à l'élément $(1,0)$ de l'ensemble d'indices de $\left(\alpha^{\prime}, \beta^{\prime}\right)$. Pour l'ordre $<^{\prime}$, on ne sait pas quel est le plus grand des indices $(1,0)$ et $(1,1)$. Supposons que $(1,0)<^{\prime}(1,1)$. Puisque $\alpha^{\prime}$ est une partition extraite de $\alpha$, on a $\alpha_{1}^{\prime} \leq \alpha_{1}$. On a dit que $\alpha_{1}+A \leq B$. A fortiori $\alpha_{1}^{\prime}+A-s<B$ et cela impose le procédé (b). Supposons maintenant que $(1,1)<^{\prime}(1,0)$. L'inégalité $\alpha_{1}+A \leq B$ entraîne $A \leq B$, a fortiori $\beta_{1}^{\prime}+B>A-s$. Cela impose encore le procédé (b), ce qui démontre l'assertion. Le procédé (b) construit le nombre $\underline{\mu}_{1}^{\prime}$ et un couple de partitions extraites $\left(\alpha^{\prime \prime}, \beta^{\prime \prime}\right)$. On a $\left(\underline{\nu}^{\prime}, \underline{\mu}^{\prime}\right)=\left(\nu^{\prime \prime},\left\{\underline{\mu}_{1}^{\prime}\right\} \sqcup \mu^{\prime \prime}\right)$, pour un couple $\left(\nu^{\prime \prime}, \mu^{\prime \prime}\right) \in P_{A-s, B-s ; s}\left(\alpha^{\prime \prime}, \beta^{\prime \prime}\right)$. On a supposé $m_{>^{\prime}} \geq 1$ mais la conclusion reste valable si $m_{>^{\prime}}=0$ : on a alors $\underline{\mu}_{1}=0,\left(\alpha^{\prime \prime}, \beta^{\prime \prime}\right)=\left(\alpha^{\prime}, \beta^{\prime}\right)$ et $\left(\nu^{\prime \prime}, \mu^{\prime \prime}\right)=\left(\underline{\nu}^{\prime}, \underline{\mu^{\prime}}\right)$. En tout cas, on a

$$
\Lambda_{A-s, B ; s}\left(\underline{\nu}^{\prime}, \underline{\mu}^{\prime}\right)=\left\{\underline{\mu}_{1}^{\prime}+B\right\} \sqcup \Lambda_{A-s, B-s ; s}\left(\nu^{\prime \prime}, \mu^{\prime \prime}\right) .
$$

L'hypothèse de récurrence entraîne que $\Lambda_{A-s, B ; s}\left(\nu^{\prime}, \mu^{\prime}\right) \leq \Lambda_{A-s, B ; s}\left(\underline{\nu}^{\prime}, \underline{\mu}^{\prime}\right)$. Avec (5), on obtient

$$
\Lambda_{A, B ; s}(\nu, \mu) \leq\left\{\alpha_{1}+\boldsymbol{\mu}_{1}+A\right\} \sqcup\left\{\underline{\mu}_{1}^{\prime}+B\right\} \sqcup \Lambda_{A-s, B-s ; s}\left(\nu^{\prime \prime}, \mu^{\prime \prime}\right) .
$$

D'après l'assertion symétrique de $1.3(9)$, on a $\mu_{1}^{\prime} \leq \boldsymbol{\mu}_{1}$. En utilisant l'inégalité $\alpha_{1}+A \leq B$, on voit que $\left\{\alpha_{1}+\boldsymbol{\mu}_{1}+A\right\} \sqcup\left\{\underline{\mu}_{1}^{\prime}+B \underline{B}\right\} \leq\left\{\alpha_{1}+\underline{\mu}_{1}^{\prime}+A\right\} \sqcup\left\{\boldsymbol{\mu}_{1}+B\right\}$. Donc

$$
\Lambda_{A, B ; s}(\nu, \mu) \leq\left\{\boldsymbol{\mu}_{1}+B\right\} \sqcup\left\{\alpha_{1}+\underline{\mu}_{1}^{\prime}+A\right\} \sqcup \Lambda_{A-s, B-s ; s}\left(\nu^{\prime \prime}, \mu^{\prime \prime}\right) .
$$

Considérons le couple $\left(\boldsymbol{\alpha}^{\prime}, \boldsymbol{\beta}^{\prime}\right)=\left(\left\{\alpha_{1}\right\} \sqcup \alpha^{\prime}, \beta^{\prime}\right)$. Symétriquement à ce qui se passait dans la première partie de la preuve, $(1,0)$ reste l'élément minimal pour l'ordre $<^{\prime}$. D'après 1.5 (4), le procédé (a) crée l'élément $\alpha_{1}+\underline{\mu}_{1}^{\prime}$ et les partitions $\left(\alpha^{\prime \prime}, \beta^{\prime \prime}\right)$. Donc le couple $\left(\left\{\alpha_{1}+\underline{\mu}_{1}^{\prime}\right\} \sqcup \nu^{\prime \prime}, \mu^{\prime \prime}\right)$ appartient à $P\left(\boldsymbol{\alpha}^{\prime}, \boldsymbol{\beta}^{\prime},<^{\prime}\right)$. On a

$$
\Lambda_{A, B-s ; s}\left(\left\{\alpha_{1}+\underline{\mu}_{1}^{\prime}\right\} \sqcup \nu^{\prime \prime}, \mu^{\prime \prime}\right)=\left\{\alpha_{1}+\underline{\mu}_{1}^{\prime}+A\right\} \sqcup \Lambda_{A-s, B-s ; s}\left(\nu^{\prime \prime}, \mu^{\prime \prime}\right) .
$$

D'où, avec (6),

$$
\Lambda_{A, B ; s}(\nu, \mu) \leq\left\{\boldsymbol{\mu}_{1}+B\right\} \sqcup \Lambda_{A, B-s ; s}\left(\left\{\alpha_{1}+\underline{\mu}_{1}^{\prime}\right\} \sqcup \nu^{\prime \prime}, \mu^{\prime \prime}\right) .
$$

Puisque $\left(\left\{\alpha_{1}+\underline{\mu}_{1}^{\prime}\right\} \sqcup \nu^{\prime \prime}, \mu^{\prime \prime}\right)$ appartient à $P\left(\boldsymbol{\alpha}^{\prime}, \boldsymbol{\beta}^{\prime},<^{\prime}\right)$, on sait par récurrence que $\Lambda_{A, B-s ; s}\left(\left\{\alpha_{1}+\underline{\mu}_{1}^{\prime}\right\} \sqcup \nu^{\prime \prime}, \mu^{\prime \prime}\right) \leq \Lambda_{A, B-s ; s}\left(\nu^{\prime}, \boldsymbol{\mu}^{\prime}\right)$. Alors

$$
\Lambda_{A, B ; s}(\nu, \mu) \leq\left\{\boldsymbol{\mu}_{1}+B\right\} \sqcup \Lambda_{A, B-s ; s}\left(\nu^{\prime}, \boldsymbol{\mu}^{\prime}\right) .
$$

En comparant avec (4), on obtient l'inégalité cherchée $\Lambda_{A, B ; s}(\nu, \mu) \leq \Lambda_{A, B ; s}(\boldsymbol{\nu}, \boldsymbol{\mu})$.

Supposons que $\Lambda_{A, B ; s}(\nu, \mu)=\Lambda_{A, B ; s}(\boldsymbol{\nu}, \boldsymbol{\mu})$. Alors toutes les inégalités ci-dessus doivent être des égalités. D'abord $\Lambda_{A-s, B ; s}\left(\nu^{\prime}, \mu^{\prime}\right)=\Lambda_{A-s, B ; s}\left(\underline{\nu}^{\prime}, \mu^{\prime}\right)$. Cela entraîne par récurrence que $\left(\nu^{\prime}, \mu^{\prime}\right) \in P_{A-s, B ; s}\left(\alpha^{\prime}, \beta^{\prime},<^{\prime}\right)$. Comme dans la première partie de la preuve, on peut alors supposer $\left(\underline{\nu}^{\prime}, \underline{\mu}^{\prime}\right)=\left(\nu^{\prime}, \mu^{\prime}\right)$. Ensuite $\left\{\alpha_{1}+\boldsymbol{\mu}_{1}+A\right\} \sqcup\left\{\underline{\mu}_{1}^{\prime}+\right.$ $B\}=\left\{\alpha_{1}+\underline{\mu}_{1}^{\prime}+A\right\} \sqcup\left\{\boldsymbol{\mu}_{1}+B\right\}$. Cela entraîne que $\alpha_{1}+A=B$ ou que $\underline{\mu}_{1}^{\prime}=\boldsymbol{\mu}_{1}$. Dans le premier cas, le procédé (a) est permis pour construire l'ensemble $P_{A, B ; s}^{a}(\alpha, \beta)$ 
et $(\nu, \mu)$ est issu de ce procédé et du couple $\left(\nu^{\prime}, \mu^{\prime}\right) \in P_{A-s, B ; s}\left(\alpha^{\prime}, \beta^{\prime},<^{\prime}\right)$. Donc $(\nu, \mu) \in P_{A, B ; s}(\alpha, \beta,<)$. Supposons $\underline{\mu}_{1}^{\prime}=\boldsymbol{\mu}_{1}$. On doit encore avoir $\Lambda_{A, B-s ; s}\left(\left\{\alpha_{1}+\right.\right.$ $\left.\left.\underline{\mu}_{1}^{\prime}\right\} \sqcup \nu^{\prime \prime}, \mu^{\prime \prime}\right)=\Lambda_{A, B-s ; s}\left(\boldsymbol{\nu}^{\prime}, \boldsymbol{\mu}^{\prime}\right)$. Par récurrence, cela entraîne que $\left(\left\{\alpha_{1}+\underline{\mu}_{1}^{\prime}\right\} \sqcup\right.$ $\left.\nu^{\prime \prime}, \mu^{\prime \prime}\right) \in P_{A, B-s ; s}\left(\boldsymbol{\alpha}^{\prime}, \boldsymbol{\beta}^{\prime},<^{\prime}\right)$. L'élément de $P_{A, B ; s}^{b}(\alpha, \beta,<)$ issu du procédé (b) et de ce couple est $\left(\left\{\alpha_{1}+\mu_{1}^{\prime}\right\} \sqcup \nu^{\prime \prime},\left\{\boldsymbol{\mu}_{1}\right\} \sqcup \mu^{\prime \prime}\right)$. Le couple $(\nu, \mu)$ est $\left(\left\{\alpha_{1}+\boldsymbol{\mu}_{1}\right\} \sqcup \nu^{\prime}, \mu^{\prime}\right)$, ou encore $\left(\left\{\alpha_{1}+\boldsymbol{\mu}_{1}\right\} \sqcup \nu^{\prime \prime},\left\{\underline{\mu}_{1}^{\prime}\right\} \sqcup \mu^{\prime \prime}\right)$. L'égalité $\underline{\mu}_{1}^{\prime}=\boldsymbol{\mu}_{1}$ entraîne que $(\nu, \mu)$ est égal au couple précédent, donc $(\nu, \mu)$ appartient à $P_{A, B ; s}(\alpha, \beta,<)$. Cela achève la démonstration.

Le lemme autorise la définition suivante.

Définition. On note $p_{A, B ; s}(\alpha, \beta,<)$ l'élément $\Lambda_{A, B ; s}(\nu, \mu)$ de $\mathcal{R}$ pour un élément quelconque $(\nu, \mu) \in P_{A, B ; s}(\alpha, \beta,<)$.

Remarque. Il résulte de 1.3(6) que, si $n_{<}=0$ ou $m_{<}=0$, on a $p_{A, B ; s}(\alpha, \beta,<)=$ $\Lambda_{A, B ; s}(\alpha, \beta)$.

\section{Propriétés des ensembles $P(\alpha, \beta,<)$}

2.1. Appartenance à $P^{b}(\alpha, \beta,<)$. Pour toute la section, on fixe un couple de partitions $(\alpha, \beta)$ et un ordre $<$ sur leur ensemble d'indices.

Lemme. Supposons $m_{<}>0$ et notons $\boldsymbol{\mu}_{1}$ le nombre construit par le procédé $(b)$. Soit $(\nu, \mu) \in P(\alpha, \beta,<)$. Supposons $\mu_{1}=\boldsymbol{\mu}_{1}$. Alors $(\nu, \mu) \in P^{b}(\alpha, \beta,<)$.

Preuve. Puisque $(\nu, \mu)$ appartient à $P^{a}(\alpha, \beta,<)$ ou à $P^{b}(\alpha, \beta,<)$, on peut supposer $(\nu, \mu) \in P^{a}(\alpha, \beta,<)$. Le procédé (a) crée le nombre $\nu_{1}$ et un couple de partitions $\left(\alpha^{\prime}, \beta^{\prime}\right)$. On a $(\nu, \mu)=\left(\left\{\nu_{1}\right\} \sqcup \nu^{\prime}, \mu^{\prime}\right)$ avec $\left(\nu^{\prime}, \mu^{\prime}\right) \in P\left(\alpha^{\prime}, \beta^{\prime},<^{\prime}\right)$. Notons $\boldsymbol{\mu}_{1}^{\prime}$ le nombre construit par le procédé (b) appliqué à $\left(\alpha^{\prime}, \beta^{\prime}\right)$. D'après l'assertion symétrique de 1.3 (9), on a les inégalités $\mu_{1}^{\prime} \leq \boldsymbol{\mu}_{1}^{\prime} \leq \boldsymbol{\mu}_{1}$. Mais $\mu_{1}^{\prime}=\mu_{1}$ et $\mu_{1}=\boldsymbol{\mu}_{1}$ par hypothèse. Donc $\mu_{1}^{\prime}=\boldsymbol{\mu}_{1}^{\prime}=\boldsymbol{\mu}_{1}$. D'après l'hypothèse $m_{<}>0$ et l'assertion symétrique de $1.3(5)$, on a $\boldsymbol{\mu}_{1} \neq 0$. Donc $\boldsymbol{\mu}_{1}^{\prime} \neq 0$ et $m_{<^{\prime}} \neq 0$ d'après l'assertion symétrique de 1.3(4). L'égalité $\mu_{1}^{\prime}=\boldsymbol{\mu}_{1}^{\prime}$ permet d'appliquer le lemme par récurrence : on a $\left(\nu^{\prime}, \mu^{\prime}\right) \in P^{b}\left(\alpha^{\prime}, \beta^{\prime},<^{\prime}\right)$. Le procédé (b) appliqué à $\left(\alpha^{\prime}, \beta^{\prime}\right)$ crée le nombre $\mu_{1}^{\prime}=\boldsymbol{\mu}_{1}$ et un couple de partitions $\left(\alpha^{\prime \prime}, \beta^{\prime \prime}\right)$. On a $\left(\nu^{\prime}, \mu^{\prime}\right)=\left(\nu^{\prime \prime},\left\{\boldsymbol{\mu}_{1}\right\} \sqcup \mu^{\prime \prime}\right)$, pour un couple $\left(\nu^{\prime \prime}, \mu^{\prime \prime}\right) \in P\left(\alpha^{\prime \prime}, \beta^{\prime \prime},<^{\prime \prime}\right)$.

Supposons $(1,0)<(1,1)$. D'après 1.3 $(10)$, le procédé $(\mathrm{b})$ appliqué à $(\alpha, \beta)$ crée le nombre $\boldsymbol{\mu}_{1}=\nu_{1}-\alpha_{1}$ et le couple de partitions $\left(\underline{\alpha}^{\prime}, \underline{\beta}^{\prime}\right)=\left(\left\{\alpha_{1}\right\} \sqcup \alpha^{\prime}, \beta^{\prime}\right)$. Pour l'ensemble d'indices de ce couple, $(1,0)$ reste l'indice minimal. Le procédé (a) appliqué à ce couple crée le nombre $\alpha_{1}+\boldsymbol{\mu}_{1}^{\prime}=\nu_{1}$ et le couple de partitions $\left(\alpha^{\prime \prime}, \beta^{\prime \prime}\right)$. Alors $\left(\left\{\nu_{1}\right\} \sqcup \nu^{\prime \prime}, \mu^{\prime \prime}\right)$ appartient à $P\left(\underline{\alpha}^{\prime}, \beta^{\prime}, \leq^{\prime}\right)$. Par le procédé (b), on en déduit le couple $\left(\left\{\nu_{1}\right\} \sqcup \nu^{\prime \prime},\left\{\boldsymbol{\mu}_{1}\right\} \sqcup \mu^{\prime \prime}\right) \in P^{b}(\alpha, \beta,<)$. Mais ce couple est égal à $(\nu, \mu)$, donc $(\nu, \mu) \in P^{b}(\alpha, \beta,<)$.

Supposons maintenant $(1,1)<(1,0)$. D'après 1.3 (10), le procédé (b) appliqué à $(\alpha, \beta)$ crée le nombre $\boldsymbol{\mu}_{1}=\beta_{1}+\nu_{1}$ et le couple de partitions $\left(\alpha^{\prime}, \beta^{\prime}-\right)$. L'indice $(1,1)$ reste minimal pour l'ordre sur l'ensemble d'indices de $\left(\alpha^{\prime}, \beta^{\prime}\right)$. Le procédé (a) appliqué à $\left(\alpha^{\prime}, \beta^{\prime}-\right)$ crée le nombre $\boldsymbol{\mu}_{1}^{\prime}-\beta_{1}=\nu_{1}$ et le couple de partitions $\left(\alpha^{\prime \prime}, \beta^{\prime \prime}\right)$. Donc $\left(\left\{\nu_{1}\right\} \sqcup \nu^{\prime \prime}, \mu^{\prime \prime}\right)$ appartient à $P\left(\alpha^{\prime}, \beta^{\prime}-,<^{\prime}-\right)$. Par le procédé $(\mathrm{b})$, on en déduit encore le couple $\left(\left\{\nu_{1}\right\} \sqcup \nu^{\prime \prime},\left\{\boldsymbol{\mu}_{1}\right\} \sqcup \mu^{\prime \prime}\right) \in P^{b}(\alpha, \beta,<)$. On conclut comme ci-dessus. 
2.2. L'ensemble $P^{b[c]}(\alpha, \beta,<)$. Soit $c \in \mathbb{N}$. On définit un sous-ensemble $P^{b[c]}(\alpha, \beta$, $<)$ de $P(\alpha, \beta,<)$ de la façon suivante. Si $m_{<}=0$, on pose $P^{b[c]}(\alpha, \beta,<)=P(\alpha, \beta,<)$. Supposons $m_{<}>0$. Si $c=0$, on pose $P^{b[0]}(\alpha, \beta,<)=P(\alpha, \beta,<)$. Supposons $c \geq 1$. Le procédé (b) construit un terme $\mu_{1}$ et un couple de partitions extraites $\left(\alpha^{\prime}, \beta^{\prime}\right)$. Alors $P^{b[c]}(\alpha, \beta,<)$ est l'ensemble des couples $\left(\nu^{\prime},\left\{\mu_{1}\right\} \sqcup \mu^{\prime}\right)$, pour $\left(\nu^{\prime}, \mu^{\prime}\right) \in$ $P^{b[c-1]}\left(\alpha^{\prime}, \beta^{\prime},<\right)$.

Considérons un couple $(\nu, \mu) \in P(\alpha, \beta,<)$ et un entier $c \geq 1$. Pour tout $d \in$ $\{1, \ldots, c\}$, on suppose donnés des réels $A(d), B(d)$. On considère la propriété

(1) $S_{d}\left(\mu+\left[B(d),-\infty[s)=S_{d}\left(p_{A(d), B(d) ; s}(\alpha, \beta,<)\right)\right.\right.$ pour tout $d \in\{1, \ldots, c\}$.

Lemme. Supposons (1) vérifiée. Alors $(\nu, \mu)$ appartient à $P^{b[c]}(\alpha, \beta,<)$.

Preuve. Si $m_{<}=0$, la conclusion est triviale. On suppose $m_{<}>0$. Supposons d'abord $c=1$ et posons pour simplifier $A=A(1), B=B(1)$. Soit $(\boldsymbol{\nu}, \boldsymbol{\mu}) \in$ $P^{b}(\alpha, \beta,<)$. Alors $\boldsymbol{\mu}_{1}$ est le nombre construit à l'aide du procédé (b). D'après l'assertion symétrique de $1.3(9)$, on a $\mu_{1} \leq \boldsymbol{\mu}_{1}$. Donc

$$
\begin{gathered}
S_{1}\left(\mu+\left[B,-\infty[s)=\mu_{1}+B \leq \boldsymbol{\mu}_{1}+B=S_{1}(\boldsymbol{\mu}+[B,-\infty[s)\right.\right. \\
\leq S_{1}\left(\Lambda_{A, B ; s}(\boldsymbol{\nu}, \boldsymbol{\mu})\right) \leq S_{1}\left(p_{A, B ; s}(\alpha, \beta,<)\right) .
\end{gathered}
$$

Les deux termes extrêmes étant égaux par hypothèse, ces inégalités sont des égalités. Donc $\mu_{1}=\boldsymbol{\mu}_{1}$. Le lemme 2.1 implique alors que $(\nu, \mu)$ appartient à $P^{b}(\alpha, \beta,<)$. Mais, pour $c=1$, on a $P^{b}(\alpha, \beta,<)=P^{b[1]}(\alpha, \beta,<)$ par définition, ce qui démontre l'assertion de l'énoncé.

Supposons maintenant $c>1$ et raisonnons par récurrence sur $c$. L'hypothèse (1) pour $c$ est plus forte que celle pour $c=1$. On applique ce que l'on vient de démontrer : $(\nu, \mu)$ appartient à $P^{b}(\alpha, \beta,<)$. Le procédé (b) crée le nombre $\mu_{1}$ et un couple de partitions extraites $\left(\alpha^{\prime}, \beta^{\prime}\right)$. On a $(\nu, \mu)=\left(\nu^{\prime},\left\{\mu_{1}\right\} \sqcup \mu^{\prime}\right)$ pour un couple $\left(\nu^{\prime}, \mu^{\prime}\right) \in P\left(\alpha^{\prime}, \beta^{\prime},<^{\prime}\right)$. Soit $d \in\{2, \ldots, c\}$. Fixons un couple $\left(\boldsymbol{\nu}^{\prime}, \boldsymbol{\mu}^{\prime}\right) \in$ $P_{A(d), B(d)-s ; s}\left(\alpha^{\prime}, \beta^{\prime},<^{\prime}\right)$. On a alors

$$
\Lambda_{A(d), B(d)-s ; s}\left(\boldsymbol{\nu}^{\prime}, \boldsymbol{\mu}^{\prime}\right)=p_{A(d), B(d)-s ; s}\left(\alpha^{\prime}, \beta^{\prime},<^{\prime}\right) .
$$

Le couple $\left(\boldsymbol{\nu}^{\prime},\left\{\mu_{1}\right\} \sqcup \boldsymbol{\mu}^{\prime}\right)$ appartient à $P(\alpha, \beta,<)$ : il est issu de $\left(\boldsymbol{\nu}^{\prime}, \boldsymbol{\mu}^{\prime}\right)$ par le procédé (b). On a la suite d'inégalités :

$$
\begin{gathered}
S_{d}\left(\mu+\left[B(d),-\infty[s)=\mu_{1}+B(d)+S_{d-1}\left(\mu^{\prime}+[B(d)-s,-\infty[s)\right.\right.\right. \\
\leq \mu_{1}+B(d)+S_{d-1}\left(\Lambda_{A(d), B(d)-s ; s}\left(\nu^{\prime}, \mu^{\prime}\right)\right) \\
\leq \mu_{1}+B(d)+S_{d-1}\left(p_{A(d), B(d)-s ; s}\left(\alpha^{\prime}, \beta^{\prime},<^{\prime}\right)\right) \\
=\mu_{1}+B(d)+S_{d-1}\left(\Lambda_{A(d), B(d)-s ; s}\left(\nu^{\prime}, \boldsymbol{\mu}^{\prime}\right)\right) .
\end{gathered}
$$

Puisque $\Lambda_{A(d), B(d) ; s}\left(\boldsymbol{\nu}^{\prime},\left\{\mu_{1}\right\} \sqcup \boldsymbol{\mu}^{\prime}\right)=\left\{\mu_{1}+B(d)\right\} \sqcup \Lambda_{A(d), B(d)-s ; s}\left(\boldsymbol{\nu}^{\prime}, \boldsymbol{\mu}^{\prime}\right)$, la suite d'inégalités se prolonge en

$$
\begin{gathered}
\mu_{1}+B(d)+S_{d-1}\left(\Lambda_{A(d), B(d)-s ; s}\left(\boldsymbol{\nu}^{\prime}, \boldsymbol{\mu}^{\prime}\right)\right) \leq S_{d}\left(\Lambda_{A(d), B(d) ; s}\left(\boldsymbol{\nu}^{\prime},\left\{\mu_{1}\right\} \sqcup \boldsymbol{\mu}^{\prime}\right)\right) \\
\leq S_{d}\left(p_{A(d), B(d) ; s}(\alpha, \beta,<)\right) .
\end{gathered}
$$

Les termes extrêmes de la suite d'inégalités obtenue sont égaux par hypothèse. Donc toutes ces inégalités sont des égalités. Cela entraîne

$$
S_{d-1}\left(\mu^{\prime}+\left[B(d)-s,-\infty[s)=S_{d-1}\left(p_{A(d), B(d)-s ; s}\left(\alpha^{\prime}, \beta^{\prime},<^{\prime}\right)\right) .\right.\right.
$$


Pour $d=1, \ldots, c-1$, posons $A^{\prime}(d)=A(d+1)$ et $B^{\prime}(d)=B(d+1)-s$. L'égalité ci-dessus se récrit

$$
S_{d}\left(\mu^{\prime}+\left[B^{\prime}(d),-\infty[s)=S_{d}\left(p_{A^{\prime}(d), B^{\prime}(d) ; s}\left(\alpha^{\prime}, \beta^{\prime},<^{\prime}\right)\right)\right.\right.
$$

pour $d=1, \ldots, c-1$. Autrement dit, $\left(\alpha^{\prime}, \beta^{\prime}\right)$ et $\left(\nu^{\prime}, \mu^{\prime}\right)$ vérifient les mêmes conditions que $(\alpha, \beta)$ et $(\nu, \mu)$, l'entier $c$ étant remplacé par $c-1$. Par récurrence, cela entraîne $\left(\nu^{\prime}, \mu^{\prime}\right) \in P^{b[c-1]}\left(\alpha^{\prime}, \beta^{\prime},<^{\prime}\right)$. Mais alors, par construction, $(\nu, \mu)$ appartient à $P^{b[c]}(\alpha, \beta,<)$.

2.3. Relation entre $P(\alpha, \beta)$ et $P\left(\alpha^{-}, \beta\right)$. On note $\mathbb{Z}^{\mathbb{N}>0}$ l'ensemble des suites infinies $\left(\lambda_{1}, \lambda_{2}, \ldots\right)$ d'entiers relatifs. Soient $x, c \in \mathbb{N}$ avec $c \geq 1$. On définit une application $\iota_{c, x}: \mathbb{Z}^{\mathbb{N}>0} \times \mathbb{Z}^{\mathbb{N}>0} \rightarrow \mathbb{Z}^{\mathbb{N}>0} \times \mathbb{Z}^{\mathbb{N}>0}$ de la façon suivante. Soit $(\underline{\nu}, \underline{\mu}) \in$ $\mathbb{Z}^{\mathbb{N}>0} \times \mathbb{Z}^{\mathbb{N}>0}$. Alors $\iota_{c, x}(\underline{\nu}, \underline{\mu})$ est l'élément $(\nu, \mu)$ de $\mathbb{Z}^{\mathbb{N}>0} \times \mathbb{Z}^{\mathbb{N}>0}$ défini par :

$$
\nu=\left(x+\underline{\mu}_{c}, \underline{\nu}_{1}, \underline{\nu}_{2}, \ldots\right), \mu=\left(\underline{\mu}_{1}, \ldots, \underline{\mu}_{c-1}, \underline{\mu}_{c+1}, \ldots\right) .
$$

Lemme. On suppose $(1,0)<(1,1)$.

(i) Pour tout $(\nu, \mu) \in P(\alpha, \beta,<)$, il existe $(\underline{\nu}, \underline{\mu}) \in P\left(\alpha^{-}, \beta,<^{-}\right)$et $c \geq 1$ de sorte que $(\nu, \mu)=\iota_{c, \alpha_{1}}(\underline{\nu}, \underline{\mu})$.

(ii) Soit $c \in \mathbb{N}_{>0}$ et soit $(\underline{\nu}, \underline{\mu}) \in P^{b[c]}\left(\alpha^{-}, \beta,<^{-}\right)$. Alors $\iota_{c, \alpha_{1}}(\underline{\nu}, \mu)$ appartient à $P(\alpha, \beta,<)$.

Preuve. Si $(\alpha, \beta)=(\emptyset, \emptyset)$, les assertions résultent de l'égalité $\iota_{c, 0}(\emptyset, \emptyset)=(\emptyset, \emptyset)$ pour tout $c$. On suppose désormais $(\alpha, \beta) \neq(\emptyset, \emptyset)$ et on raisonne par récurrence sur $n_{<}+m_{<}$.

Prouvons (i). Soit $(\nu, \mu) \in P(\alpha, \beta,<)$. Supposons que $(\nu, \mu) \in P^{a}(\alpha, \beta,<)$. Le procédé (a) crée le nombre $\nu_{1}$ et des partitions extraites $\left(\alpha^{\prime}, \beta^{\prime}\right)$. On a $\nu=\left\{\nu_{1}\right\} \sqcup$ $\nu^{\prime}, \mu=\mu^{\prime}$ pour un couple $\left(\nu^{\prime}, \mu^{\prime}\right) \in P\left(\alpha^{\prime}, \beta^{\prime},<\right)$. D'après [1.5 (4), le procédé (b) appliqué à $\left(\alpha^{-}, \beta\right)$ crée le nombre $\underline{\mu}_{1}=\nu_{1}-\alpha_{1}$ et les mêmes partitions $\left(\alpha^{\prime}, \beta^{\prime}\right)$. En conséquence, le couple $(\underline{\nu}, \mu)=\left(\nu^{\prime},\left\{\nu_{1}-\alpha_{1}\right\} \sqcup \mu^{\prime}\right)$ appartient à $P\left(\alpha^{-}, \beta,<^{-}\right)$. On vérifie que $(\nu, \mu)=\iota_{1, \alpha_{1}}(\underline{\nu}, \underline{\mu})$.

Supposons au contraire que $(\nu, \mu) \in P^{b}(\alpha, \beta,<)$. Le procédé (b) crée le nombre $\mu_{1}$ et des partitions $\left(\alpha^{\prime}, \beta^{\prime}\right)$. L'hypothèse $(1,0)<(1,1)$ implique que le plus grand terme de $\alpha^{\prime}$ est $\alpha_{1}$ et que $(1,0)$ est encore le plus petit élément pour l'ordre $<^{\prime}$. On a $\nu=\nu^{\prime}, \mu=\left\{\mu_{1}\right\} \sqcup \mu^{\prime}$, pour un couple $\left(\nu^{\prime}, \mu^{\prime}\right) \in P\left(\alpha^{\prime}, \beta^{\prime},<^{\prime}\right)$. Par hypothèse de récurrence, il existe $c \geq 1$ et $\left(\underline{\nu}^{\prime}, \underline{\mu}^{\prime}\right) \in P\left(\alpha^{\prime}-, \beta^{\prime},<^{\prime}-\right)$ de sorte que $\left(\nu^{\prime}, \mu^{\prime}\right)=$ $\iota_{c, \alpha_{1}}\left(\underline{\nu}^{\prime}, \underline{\mu}^{\prime}\right)$. D'après $1.5(7)$ le procédé (b) appliqué à $\left(\alpha^{-}, \beta\right)$ crée le même nombre $\mu_{1}$ et les partitions $\left(\alpha^{\prime}-, \beta^{\prime}\right)$. En conséquence, le couple $(\underline{\nu}, \underline{\mu})=\left(\underline{\nu^{\prime}},\left\{\mu_{1}\right\} \sqcup \underline{\mu}^{\prime}\right)$ appartient à $P\left(\alpha^{-}, \beta\right)$. Mais on voit que $(\nu, \mu)=\iota_{c+1, \alpha_{1}}(\underline{\nu}, \underline{\mu})$. Cela achève la preuve de (i).

Prouvons (ii). Le procédé (b) appliqué à $\left(\alpha^{-}, \beta\right)$ crée un nombre $\mu_{1}$ et des partitions extraites $\left(\alpha^{\prime}, \beta^{\prime}\right)$. On a $\underline{\nu}=\nu^{\prime}, \underline{\mu}=\left\{\mu_{1}\right\} \sqcup \mu^{\prime}$ pour un élément $\left(\nu^{\prime}, \mu^{\prime}\right) \in$ $P^{b[c-1]}\left(\alpha^{\prime}, \beta^{\prime},<^{\prime}\right)$. En effet, si $m_{<^{-}}>0$, cela résulte de l'hypothèse $(\underline{\nu}, \underline{\mu}) \in P^{b[c]}\left(\alpha^{-}\right.$, $\left.\beta,<^{-}\right)$et de la définition de ce dernier ensemble. Si au contraire $m_{<^{-}}=0$, on a $\mu_{1}=0$ et $\left(\alpha^{\prime}, \beta^{\prime}\right)=\left(\alpha^{-}, \beta\right)$ et il suffit de prendre $\left(\nu^{\prime}, \mu^{\prime}\right)=(\underline{\nu}, \underline{\mu})$. Supposons d'abord $c=1$. Parce que $(1,0)<(1,1)$ et d'après 1.5] (4), le procédé (a) appliqué à $(\alpha, \beta)$ crée le nombre $\nu_{1}=\alpha_{1}+\mu_{1}$ et les mêmes partitions $\left(\alpha^{\prime}, \beta^{\prime}\right)$. Alors le couple $\left(\nu_{1} \sqcup \nu^{\prime}, \mu^{\prime}\right)$ appartient à $P(\alpha, \beta,<)$. Or ce couple est égal à $\iota_{1, \alpha_{1}}(\underline{\nu}, \underline{\mu})$. Supposons maintenant $c \geq 2$. Toujours parce que $(1,0)<(1,1)$ et d'après [1.5)(7), le 
procédé $(\mathrm{b})$ appliqué à $(\alpha, \beta)$ crée le même élément $\mu_{1}$ et le couple de partitions $\left(\alpha^{\prime \prime}, \beta^{\prime \prime}\right)=\left(\left\{\alpha_{1}\right\} \sqcup \alpha^{\prime}, \beta^{\prime}\right)$. On a $\alpha^{\prime}=\alpha^{\prime \prime}-$. On applique le lemme par récurrence : l'élément $\left(\underline{\nu}^{\prime \prime}, \mu^{\prime \prime}\right)=\iota_{c-1, \alpha_{1}}\left(\nu^{\prime}, \mu^{\prime}\right)$ appartient à $P\left(\alpha^{\prime \prime}, \beta^{\prime \prime},<^{\prime \prime}\right)$. Alors, par le procédé (b), le couple $\overline{(} \nu, \mu)=\left(\underline{\nu}^{\prime \prime},\left\{\mu_{1}\right\} \sqcup \underline{\mu}^{\prime \prime}\right)$ appartient à $P(\alpha, \beta,<)$. On vérifie l'égalité $(\nu, \mu)=\iota_{c, \alpha_{1}}(\underline{\nu}, \underline{\mu})$. Cela achève la démonstration.

2.4. Nombres d'éléments "de type $b$ ". Introduisons l'élément canonique de type (b) $(\nu, \mu) \in P_{A, B ; s}(\alpha, \beta,<)$. Soit $c \in \mathbb{N}_{>0}$. Il existe des entiers $a, b \in \mathbb{N}$ tels que $a+b=c$, de sorte que l'ensemble des $c$ plus grands éléments de $p_{A, B ; s}(\alpha, \beta,<)=$ $\Lambda_{A, B ; s}(\nu, \mu)$ soit la réunion de celui des $a$ plus grands termes de $\nu \sqcup+[A,-\infty[$ s et de celui des $b$ plus grands termes de $\mu+\left[B,-\infty\left[{ }_{s}\right.\right.$. Les entiers $a$ et $b$ ne sont pas complètement déterminés car les deux éléments de $\mathcal{R}$ intervenant peuvent avoir des termes communs : si par exemple le $a$-ième terme de $\nu \sqcup+\left[A,-\infty\left[{ }_{s}\right.\right.$ est égal au $(b+1)-$ ième terme de $\mu+[B,-\infty[s$, le couple $(a-1, b+1)$ convient lui-aussi. On choisit $a$ et $b$ de sorte que $b$ soit le plus grand possible. On note alors $b_{A, B ; s}(\alpha, \beta,<; c)=b$.

Lemme. (i) Pour tout réel $\underline{B}$ tel que $B \leq \underline{B} \leq B+s$, on a les inégalités

$$
b_{A, B ; s}(\alpha, \beta,<; c) \leq b_{A, \underline{B} ; s}(\alpha, \beta,<; c) \leq b_{A, B ; s}(\alpha, \beta,<; c)+1
$$

pour tout $c \geq 1$.

(ii) Pour tout $c \geq 1$ et pour tout entier $\underline{b} \in\left\{b_{A, B ; s}(\alpha, \beta,<; c), \ldots, c\right\}$, il existe un réel $\underline{B} \geq B$ tel que $b_{A, \underline{B} ; s}(\alpha, \beta,<; c)=\underline{b}$.

Preuve de (i). Fixons $c \geq 1$, posons $b=b_{A, B ; s}(\alpha, \beta,<; c), \underline{b}=b_{A, \underline{B} ; s}(\alpha, \beta,<; c)$. Introduisons les éléments canoniques de type (b) $(\nu, \mu) \in P_{A, B ; s}(\alpha, \beta,<)$ et $(\underline{\nu}, \underline{\mu}) \in$ $P_{A, \underline{B} ; s}(\alpha, \beta,<)$.

Supposons d'abord $n_{<}=0$ ou $m_{<}=0$. Alors $(\nu, \mu)=(\underline{\nu}, \mu)=(\alpha, \beta)$. Posons $\Lambda^{0}=\alpha+\left[A,-\infty\left[s, \Lambda^{1}=\beta+\left[B,-\infty\left[{ }_{s}, \underline{\Lambda}^{1}=\beta+[\underline{B},-\infty[s\right.\right.\right.\right.$. Par définition, $b$, resp. $\underline{b}$, est le plus grand entier $i \in\{0, \ldots, c\}$ tel que $\Lambda_{i}^{1} \geq \Lambda_{c+1-i}^{0}$, resp. $\underline{\Lambda}_{i}^{1} \geq \Lambda_{c+1-i}^{0}$. En particulier $\Lambda_{b}^{1} \geq \Lambda_{c+1-b}^{0}$. Puisque $\underline{B} \geq B$, on a $\underline{\Lambda}_{b}^{1}=\Lambda_{b}^{1}+\underline{B}-B \geq \Lambda_{b}^{1} \geq \Lambda_{c+1-b}^{0}$, ce qui, d'après la caractérisation ci-dessus, entraîne $\underline{b} \geq b$. Si $b \geq c-1$, on a forcément $\underline{b} \leq b+1$ puisque $\underline{b} \leq c$. Si $b<c-1$, on a $\Lambda_{b+1}^{1}<\Lambda_{c-b}^{0}$. On a alors

$$
\underline{\Lambda}_{b+2}^{1} \leq \underline{\Lambda}_{b+1}^{1}-s=\Lambda_{b+1}^{1}+\underline{B}-B-s<\Lambda_{c-b}^{0}<\Lambda_{c-b-1}^{0},
$$

donc encore $\underline{b} \leq b+1$ d'après la caractérisation ci-dessus.

Supposons maintenant $n_{<}>0$ et $m_{<}>0$. Supposons d'abord que les éléments $(\nu, \mu)$ et $(\underline{\nu}, \underline{\mu})$ soient construits à l'aide du même procédé, par exemple le procédé (b). Ce procédé crée un nombre $\mu_{1}=\underline{\mu}_{1}$ et des partitions $\left(\alpha^{\prime}, \beta^{\prime}\right)$. On a $(\nu, \mu)=$ $\left(\nu^{\prime},\left\{\mu_{1}\right\} \sqcup \mu^{\prime}\right)$ où $\left(\nu^{\prime}, \mu^{\prime}\right)$ est l'élément canonique de type (b) de $P_{A, B-s ; s}\left(\alpha^{\prime}, \beta^{\prime},<\right)$. D'où

$$
\nu+\left[A,-\infty\left[{ }_{s}=\nu^{\prime}+\left[A,-\infty\left[{ }_{s}, \mu+\left[B,-\infty\left[{ }_{s}=\left\{\mu_{1}+B\right\} \sqcup\left(\mu^{\prime}+\left[B-s,-\infty\left[{ }_{s}\right) .\right.\right.\right.\right.\right.\right.\right.\right.
$$

D'après 1.4 (6), $\mu_{1}+B$ est le plus grand élément de la réunion des deux suites cidessus. Il en résulte que $b=1$ si $c=1$ tandis que $b=1+b_{A, B-s ; s}\left(\alpha^{\prime}, \beta^{\prime},<^{\prime} ; c-1\right)$ si $c \geq 2$. Le même résultat vaut pour $\underline{b}: \underline{b}=1$ si $c=1$ tandis que $\underline{b}=1+$ $b_{A, \underline{B}-s ; s}\left(\alpha^{\prime}, \beta^{\prime},<^{\prime} ; c-1\right)$ si $k \geq 2$. Le résultat cherché s'en déduit immédiatement si $c=1$ et par récurrence si $c \geq 2$.

On suppose maintenant que les éléments $(\nu, \mu)$ et $(\underline{\nu}, \mu)$ sont construits à l'aide de procédés différents. Supposons d'abord $(1,0)<(1,1)$. Si $(\nu, \mu)$ est construit à l'aide du procédé (b), on a $\alpha_{1}+A \leq B$. Mais alors $\alpha_{1}+A \leq \underline{B}$ et $(\underline{\nu}, \underline{\mu})$ est aussi 
construit à l'aide du procédé (b), contrairement à l'hypothèse (si $\alpha_{1}+A=\underline{B}$, la construction de l'élément canonique de type (b) privilégie le procédé (b)). Donc $(\nu, \mu)$ est construit à l'aide du procédé (a). On a donc $\alpha_{1}+A>B$ (on ne peut pas avoir égalité pour la raison que l'on vient de donner). L'élément $(\underline{\nu}, \underline{\mu})$ est construit à l'aide du procédé (b) donc $\alpha_{1}+A \leq \underline{B}$. Le procédé (a) construit le nombre $\nu_{1}$ et des partitions $\left(\alpha^{\prime}, \beta^{\prime}\right)$. Puisque $(1,0)<(1,1)$, le procédé (b) construit d'après 1.3)(10) le nombre $\underline{\mu}_{1}$ et les partitions $\left(\underline{\alpha}^{\prime}, \underline{\beta}^{\prime}\right)=\left(\left\{\alpha_{1}\right\} \sqcup \alpha^{\prime}, \beta^{\prime}\right)$. On obtient

$$
\nu+\left[A,-\infty\left[{ }_{s}=\left\{\nu_{1}+A\right\} \sqcup\left(\nu^{\prime}+\left[A-s,-\infty[s), \mu+\left[B,-\infty\left[{ }_{s}=\mu^{\prime}+\left[B,-\infty\left[{ }_{s},\right.\right.\right.\right.\right.\right.\right.\right.
$$

où $\left(\nu^{\prime}, \mu^{\prime}\right)$ est l'élément canonique de type (b) de $P_{A-s, B ; s}\left(\alpha^{\prime}, \beta^{\prime},<^{\prime}\right)$. Comme plus haut, $\nu_{1}+A$ est le plus grand élément de la réunion des deux suites ci-dessus. Plus précisément, il est strictement supérieur aux autres d'après 1.4(7). De même,

$$
\underline{\nu}+\left[A,-\infty\left[{ }_{s}=\underline{\nu}^{\prime}+\left[A,-\infty\left[s, \underline{\mu}+\left[\underline{B},-\infty\left[{ }_{s}=\left\{\underline{\mu}_{1}+\underline{B}\right\} \sqcup\left(\underline{\mu}^{\prime}+[\underline{B}-s,-\infty[s),\right.\right.\right.\right.\right.\right.\right.
$$

où $\left(\underline{\nu}^{\prime}, \mu^{\prime}\right)$ est l'élément canonique de type (b) de $P_{A, \underline{B}-s ; s}\left(\underline{\alpha}^{\prime}, \beta^{\prime}, \underline{\leq}^{\prime}\right)$. Le nombre $\underline{\mu}_{1}+\underline{B}$ est le plus grand élément de la réunion des deux suites ci-dessus.

Appliquons le procédé (b) au couple $\left(\alpha^{\prime}, \beta^{\prime}\right)$. Il crée un nombre que nous notons par anticipation $\mu_{1}$ et un couple de partitions $\left(\alpha^{\prime \prime}, \beta^{\prime \prime}\right)$. Notons $\left(\nu^{\prime \prime}, \mu^{\prime \prime}\right)$ l'élément canonique de type (b) de $P_{A-s, B-s ; s}\left(\alpha^{\prime \prime}, \beta^{\prime \prime},<^{\prime \prime}\right)$. Montrons que

(3) on a $\left(\nu^{\prime}, \mu^{\prime}\right)=\left(\nu^{\prime \prime},\left\{\mu_{1}\right\} \sqcup \mu^{\prime \prime}\right)$ et $\mu_{1}+B$ est le plus grand élément de $\Lambda_{A-s, B ; s}\left(\nu^{\prime}, \mu^{\prime}\right)$.

Supposons d'abord $m_{<^{\prime}}>0$. Si $(1,0)<^{\prime}(1,1)$, l'inégalité $\alpha_{1}+A \leq \underline{B}$ entraîne $\alpha_{1}^{\prime}+A-s \leq \alpha_{1}+A-s \leq \underline{B}-s \leq B$ et, par définition, $\left(\nu^{\prime}, \mu^{\prime}\right)$ est construit à l'aide du procédé $(\mathrm{b})$, d'où la première assertion de $(3)$. Si $(1,1)<^{\prime}(1,0)$, la même inégalité entraîne $\beta_{1}^{\prime}+B \geq \underline{B}-s \geq \alpha_{1}+A-s \geq A-s$ et la même conclusion. Supposons maintenant $m_{<^{\prime}}=0$. Alors $\beta^{\prime}=\emptyset, \mu_{1}=0$ et $\left(\alpha^{\prime \prime}, \beta^{\prime \prime}\right)=\left(\alpha^{\prime}, \beta^{\prime}\right)$. La première assertion de $(3)$ est alors évidente. Si $m_{<^{\prime}}>0$ et $n_{<^{\prime}}>0$, la seconde assertion de (3) résulte de 1.4(6). Si $m_{<^{\prime}}=0$ ou $n_{<^{\prime}}=0$, on a $\left(\nu^{\prime}, \mu^{\prime}\right)=\left(\alpha^{\prime}, \beta^{\prime}\right)$ et la seconde assertion se résume à l'inégalité $\mu_{1}+B \geq \alpha_{1}^{\prime}+A-s$. Celle-ci résulte de la suite d'inégalités

$$
\mu_{1}+B \geq B \geq \underline{B}-s \geq \alpha_{1}+A-s \geq \alpha_{1}^{\prime}+A-s .
$$

Cela prouve (3).

Notons que $(1,0)$ reste le plus petit élément pour l'ordre $\leq^{\prime}$. Le procédé (a) appliqué à $\left(\underline{\alpha}^{\prime}, \underline{\beta}^{\prime}\right)$ crée un nombre que nous notons par anticipation $\underline{\nu}_{1}$ et le même couple $\left(\alpha^{\prime \prime}, \beta^{\prime \prime}\right)$ d'après $1.5(4)$. Notons $\left(\underline{\nu}^{\prime \prime}, \underline{\mu}^{\prime \prime}\right)$ l'élément canonique de type (b) de $P_{A-s, \underline{B}-s ; s}\left(\alpha^{\prime \prime}, \beta^{\prime \prime},<^{\prime \prime}\right)$. Montrons que

(4) on a $\left(\underline{\nu}^{\prime}, \underline{\mu}^{\prime}\right)=\left(\left\{\underline{\nu}_{1}\right\} \sqcup \underline{\nu}^{\prime \prime}, \underline{\mu}^{\prime \prime}\right)$ et $\underline{\nu}_{1}+A$ est strictement plus grand que les éléments de $\Lambda_{A-s, \underline{B}-s ; s}\left(\underline{\nu}^{\prime \prime}, \underline{\mu}^{\prime \prime}\right)$.

Supposons d'abord $n_{\leq^{\prime}}>0$. On a $(1,0) \leq^{\prime}(1,1)$. L'inégalité $\alpha_{1}+A>B$ entraîne $\alpha_{1}+A>\underline{B}-s$ et, par définition, $\left(\underline{\nu}^{\prime}, \underline{\mu}^{\prime}\right)$ est construit par le procédé (a), d'où la première assertion de (4). Supposons maintenant $n_{\leq^{\prime}}=0$. Alors $\underline{\alpha}^{\prime}=\emptyset, \underline{\nu}_{1}=0$ et $\left(\alpha^{\prime \prime}, \beta^{\prime \prime}\right)=\left(\underline{\alpha}^{\prime}, \underline{\beta}^{\prime}\right)$. La première assertion de (4) est alors évidente. Si $n_{\leq^{\prime}}>0$ et $m_{\leq^{\prime}}>0$, la seconde assertion de (4) résulte de 1.4(7). Supposons $n_{\leq^{\prime}}=0$ ou $m_{\leq^{\prime}}=0$. Cette hypothèse entraîne $m_{\leq^{\prime}}=0$ d'après [1.2(1). Cette dernière égalité entraîne $\underline{\beta}^{\prime}=\emptyset$ et $\left(\underline{\nu}^{\prime}, \underline{\mu}^{\prime}\right)=\left(\underline{\alpha}^{\prime}, \emptyset\right)$. La seconde assertion de (4) se résume à l'inégalité $\underline{\nu}_{1}+\bar{A}>\underline{B}-s$. Par construction, $\underline{\nu}_{1} \geq \underline{\alpha}_{1}^{\prime}=\alpha_{1}$. D'où $\underline{\nu}_{1}+A \geq \alpha_{1}+A>$ $B \geq \underline{B}-s$, ce qui achève de prouver (4). 
Supposons maintenant $(1,1)<(1,0)$. Si $(\nu, \mu)$ est construit à l'aide du procédé (b), on a $\beta_{1}+B \geq A$. Mais alors $\beta_{1}+\underline{B} \geq A$ et $(\underline{\nu}, \mu)$ est aussi construit à l'aide du procédé (b), contrairement à l'hypothèse. Donc $(\nu, \mu)$ est construit à l'aide du procédé (a). On a donc $\beta_{1}+B<A$ (on ne peut pas avoir égalité pour la raison donnée plus haut). L'élément $(\underline{\nu}, \mu)$ est construit à l'aide du procédé (b) donc $\beta_{1}+\underline{B} \geq A$. Le procédé (a) construit le nombre $\nu_{1}$ et des partitions $\left(\alpha^{\prime}, \beta^{\prime}\right)$. Puisque $(1,1)<(1,0)$, le procédé (b) construit d'après 1.3 (10) le nombre $\underline{\mu}_{1}$ et les partitions $\left(\underline{\alpha}^{\prime}, \beta^{\prime}\right)=\left(\alpha^{\prime}, \beta^{\prime}-\right)$. On obtient les mêmes relations (1) et (2) que plus haut, avec les mêmes propriétés.

Notons que $(1,1)$ reste le plus petit élément pour l'ordre $<^{\prime}$. Appliquons le procédé (b) au couple $\left(\alpha^{\prime}, \beta^{\prime}\right)$. Il crée le nombre $\mu_{1}$ et un couple de partitions $\left(\alpha^{\prime \prime}, \beta^{\prime \prime}\right)$. Notons $\left(\nu^{\prime \prime}, \mu^{\prime \prime}\right)$ l'élément canonique de type (b) de $P_{A-s, B-s ; s}\left(\alpha^{\prime \prime}, \beta^{\prime \prime},<^{\prime \prime}\right)$. On démontre encore la relation (3) de la même façon que l'on a démontré (4) cidessus : l'utilisation des inégalités $\alpha_{1}+A>B$ et $\alpha_{1}+A \leq \underline{B}$ est remplacée par celle des inégalités $\beta_{1}+\underline{B} \geq A$ et $\beta_{1}+B<A$. Appliquons le procédé (a) au couple $\left(\underline{\alpha}^{\prime}, \beta^{\prime}\right)$. Il crée le nombre $\underline{\nu}_{1}$ et le même couple de partitions $\left(\alpha^{\prime \prime}, \beta^{\prime \prime}\right)$. Notons $\left(\underline{\nu}^{\prime \prime}, \bar{\mu}^{\prime \prime}\right)$ l'élément canonique de type (b) de $P_{A-s, B-s ; s}\left(\alpha^{\prime \prime}, \beta^{\prime \prime},<^{\prime \prime}\right)$. On démontre encore (4) de même que l'on a démontré (3) ci-dessus.

Ces relations (1) à (4) sont donc démontrées en tout cas. En utilisant (1) et (3) et les propriétés de maximalité de $\nu_{1}+A$ et $\mu_{1}+B$ que l'on a indiquées, on voit que $b=0$ si $c=1, b=1$ si $c=2$ et $b=1+b_{A-s, B-s ; s}\left(\alpha^{\prime \prime}, \beta^{\prime \prime},<^{\prime \prime} ; c-2\right)$ si $c \geq 3$. De même, en utilisant (2) et (4) et les propriétés de maximalité de $\underline{\mu}_{1}+\underline{B}$ et $\underline{\nu}_{1}+A$, on voit que $\underline{b}=1$ si $c=1$ ou $c=2, \underline{b}=1+b_{A-s, \underline{B}-s ; s}\left(\alpha^{\prime \prime}, \beta^{\prime \prime},<^{\prime \prime} ; c-2\right)$ si $c \geq 3$. L'assertion (i) de l'énoncé en résulte, par récurrence dans le cas où $c \geq 3$.

Cette assertion (i) entraîne que l'image de la suite $i \mapsto b_{A, B+i s ; s}(\alpha, \beta,<; c)$, pour $i \in \mathbb{N}$, est un intervalle d'entiers contenant $b_{A, B ; s}(\alpha, \beta,<; c)$. Pour démontrer (ii), il suffit de prouver que cette image contient $c$. Puisque l'ensemble $P(\alpha, \beta,<)$ est fini, on peut choisir un entier $h$ tel que $h \geq \nu_{i}$ et $h \geq \mu_{i}$ pour tout $(\nu, \mu) \in P(\alpha, \beta,<)$ et tout $i \geq 1$. Choisissons $\underline{B}$ tel que $\underline{B}+s-s c>h+A$. Considérons l'élément canonique de type (b) $(\nu, \mu) \in P_{A, \underline{B} ; s}(\alpha, \beta,<)$. Le $c$-ième terme de $\mu+\left[\underline{B},-\infty\left[{ }_{s}\right.\right.$ est $\mu_{c}+\underline{B}+s-s c$. Il est strictement supérieur à $\nu_{1}+A$ qui est le premier terme de $\nu+\left[\bar{A},-\infty\left[s\right.\right.$. On a alors $b_{A, \underline{B} ; s}(\alpha, \beta,<; c)=c$ par définition de $b_{A, \underline{B} ; s}(\alpha, \beta,<; c)$. Cela achève la preuve.

\subsection{Premier lemme de majoration.}

Lemme. On suppose $(1,0)<(1,1)$. Pour tout $c \in \mathbb{N}_{>0}$, on a l'inégalité

$$
S_{c}\left(p_{A, B ; s}\left(\alpha^{-}, \beta,<^{-}\right)\right) \leq S_{c}\left(p_{A, B ; s}(\alpha, \beta,<) \leq S_{c}\left(p_{A, B ; s}\left(\alpha^{-}, \beta,<^{-}\right)\right)+\alpha_{1}+\beta_{1} .\right.
$$

Il y a un lemme symétrique en échangeant les rôles de $\alpha$ et $\beta$. On doit alors supposer $(1,1)<(1,0)$. La démonstration de ces lemmes sera donnée en 2.7 .

\subsection{Deuxième lemme de majoration.}

Lemme. Pour tout $c \in \mathbb{N}_{>0}$, on a l'inégalité

$$
S_{c}\left(p_{A, B-s ; s}(\alpha, \beta,<) \leq S_{c}\left(p_{A-s, B ; s}(\alpha, \beta,<)\right)+\sup \left(\alpha_{1}+A-B, 0\right) .\right.
$$

Là encore, il y a un symétrique de ce lemme obtenu en échangeant les rôles de $\alpha$ et $\beta$ et, simultanément, ceux de $A$ et $B$. La démonstration sera donnée en 2.8 .

Pour démontrer ce lemme, le lemme précédent et leurs symétriques, on raisonne par récurrence sur $n_{<}+m_{<}$. C'est-à-dire que l'on vérifiera les quatre lemmes dans 
le cas $n_{<}=m_{<}=0$ (en fait dans les cas $n_{<}=0$ ou $m_{<}=0$ ). Ensuite, on supposera que $n_{<}+m_{<}>0$ et que les quatre lemmes sont vérifiés pour des couples $\left(\alpha^{\prime}, \beta^{\prime}\right)$ tels que $n_{<^{\prime}}+m_{<^{\prime}}<n_{<}+m_{<}$. On ne fera les démonstrations que des lemmes énoncés, celles des symétriques étant évidemment analogues.

2.7. Preuve du lemme 2.5. Supposons d'abord $m_{<}=0$. A fortiori $m_{<^{-}}=0$. Alors $\beta=\emptyset, p_{A, B ; s}(\alpha, \beta,<)=\left(\alpha+\left[A,-\infty[s) \sqcup\left[B,-\infty\left[{ }_{s}\right.\right.\right.\right.$ et $p_{A, B ; s}\left(\alpha^{-}, \beta,<^{-}\right)=$ $\left(\alpha^{-}+\left[A,-\infty\left[{ }_{s}\right) \sqcup\left[B,-\infty\left[{ }_{s}\right.\right.\right.\right.$. On a $\alpha^{-} \leq \alpha$, d'où $\alpha^{-}+\left[A,-\infty\left[{ }_{s} \leq \alpha+\left[A,-\infty\left[{ }_{s}\right.\right.\right.\right.$, puis $p_{A, B ; s}\left(\alpha^{-}, \beta,<^{-}\right) \leq p_{A, B ; s}(\alpha, \beta,<)$ d'après le lemme 1.1. La première inégalité de l'énoncé en résulte. Soient $a, b \in \mathbb{N}$ tels que $a+b=c$ et

$$
S_{c}\left(p_{A, B ; s}(\alpha, \beta,<)\right)=S_{a}\left(\alpha+\left[A,-\infty[s)+S_{b}([B,-\infty[s) .\right.\right.
$$

On vérifie que $S_{a}\left(\alpha+\left[A,-\infty[s)=S_{a}\left(\alpha^{-}+\left[A,-\infty[s)+\alpha_{1}-\alpha_{a+1} \leq S_{a}\left(\alpha^{-}+\right.\right.\right.\right.\right.$ $\left[A,-\infty[s)+\alpha_{1}\right.$. Alors

$$
\begin{aligned}
S_{c}\left(p_{A, B ; s}(\alpha, \beta,<)\right) & \leq S_{a}\left(\alpha^{-}+\left[A,-\infty[s)+S_{b}\left(\left[B,-\infty\left[_{s}\right)+\alpha_{1}\right.\right.\right.\right. \\
\leq & S_{c}\left(p_{A, B ; s}\left(\alpha, \beta,<^{-}\right)\right)+\alpha_{1} .
\end{aligned}
$$

D'où la seconde inégalité de l'énoncé.

On suppose maintenant $m_{<}>0$. En vertu de 1.2(1) et 1.5(1), cela entraîne $n_{<}>0$ et $m_{<^{-}}>0$. Considérons d'abord le cas particulier où $n_{<^{-}}=0$. On a décrit l'ensemble $P(\alpha, \beta,<)$ en 1.5)(2) et on utilise les notations de cette description. On a $\alpha^{-}=\emptyset$ donc $p_{A, B ; s}\left(\alpha^{-}, \beta,<^{-}\right)=\left[A,-\infty\left[{ }_{s} \sqcup\left(\beta+\left[B,-\infty\left[{ }_{s}\right)\right.\right.\right.\right.$. Evidemment $\left[A,-\infty\left[{ }_{s} \leq \nu^{m+1}+\left[A,-\infty\left[{ }_{s}\right.\right.\right.\right.$ et $\beta+\left[B,-\infty\left[{ }_{s} \leq \mu^{m+1}+\left[B,-\infty\left[{ }_{s}\right.\right.\right.\right.$ puisque $\mu^{m+1}=\beta$. D'après le lemme 1.1, on a donc

$$
p_{A, B ; s}\left(\alpha^{-}, \beta,<^{-}\right) \leq \Lambda_{A, B ; s}\left(\nu^{m+1}, \mu^{m+1}\right) \leq p_{A, B ; s}(\alpha, \beta,<) .
$$

D'où la première inégalité de l'énoncé. Choisissons $i$ tel que $p_{A, B ; s}(\alpha, \beta,<)=$ $\Lambda_{A, B ; s}\left(\nu^{i}, \mu^{i}\right)$. Soient $a, b \in \mathbb{N}$ tels que $a+b=c$ et

$$
S_{c}\left(\Lambda_{A, B ; s}\left(\nu^{i}, \mu^{i}\right)\right)=S_{a}\left(\nu^{i}+\left[A,-\infty[s)+S_{b}\left(\mu^{i}+[B,-\infty[s) .\right.\right.\right.
$$

On a $S_{a}\left(\nu^{i}+\left[A,-\infty[s) \leq \alpha_{1}+\beta_{i}+S_{a}\left(\left[A,-\infty[s) \leq \alpha_{1}+\beta_{1}+S_{a}([A,-\infty[s)\right.\right.\right.\right.$ et, puisque $\mu^{i} \leq \beta, S_{b}\left(\mu^{i}+\left[B,-\infty[s) \leq S_{b}(\beta+[B,-\infty[s)\right.\right.$. D'où

$$
\begin{aligned}
S_{c}\left(\Lambda_{A, B ; s}\left(\nu^{i}, \mu^{i}\right)\right) & \leq \alpha_{1}+\beta_{1}+S_{a}\left(\left[A,-\infty[s)+S_{b}(\beta+[B,-\infty[s)\right.\right. \\
& \leq \alpha_{1}+\beta_{1}+S_{c}\left(\Lambda_{A, B ; s}(\emptyset, \beta)\right),
\end{aligned}
$$

ce qui équivaut à

$$
S_{c}\left(p_{A, B ; s}(\alpha, \beta,<)\right) \leq \alpha_{1}+\beta_{1}+S_{c}\left(p_{A, B ; s}\left(\alpha^{-}, \beta,<^{-}\right)\right) .
$$

C'est la seconde inégalité de l'énoncé.

On suppose désormais $n_{<}>0, m_{<}>0, n_{<-}>0, m_{<-}>0$. Supposons d'abord $\alpha_{1}+A \leq B$, donc l'ensemble $P_{A, B ; s}^{b}(\alpha, \beta,<)$ n'est pas vide. D'après [1.5](8), l'ensemble $P_{A, B ; s}^{b}\left(\alpha^{-}, \beta,<^{-}\right)$n'est pas vide non plus. On applique le procédé (b) à $(\alpha, \beta)$. Il crée un nombre $\mu_{1}$ et des partitions $\left(\alpha^{\prime}, \beta^{\prime}\right)$. Un élément de $P_{A, B ; s}^{b}(\alpha, \beta,<)$ est de la forme $\left(\nu^{\prime},\left\{\mu_{1}\right\} \sqcup \mu^{\prime}\right)$ où $\left(\nu^{\prime}, \mu^{\prime}\right) \in P_{A, B-s ; s}\left(\alpha^{\prime}, \beta^{\prime},<^{\prime}\right)$. D'où

$$
p_{A, B ; s}(\alpha, \beta,<)=\left\{\mu_{1}+B\right\} \sqcup p_{A, B-s ; s}\left(\alpha^{\prime}, \beta^{\prime},<^{\prime}\right) .
$$

De plus, $\mu_{1}+B$ est le plus grand terme de $p_{A, B ; s}(\alpha, \beta,<)$ d'après 1.4(6). Donc

$$
S_{c}\left(p_{A, B ; s}(\alpha, \beta,<)\right)=\left\{\begin{array}{cl}
\mu_{1}+B, & \text { si } c=1 \\
\mu_{1}+B+S_{c-1}\left(p_{A, B-s ; s}\left(\alpha^{\prime}, \beta^{\prime},<^{\prime}\right)\right), & \text { si } c \geq 2 .
\end{array}\right.
$$


On applique le procédé (b) à $\left(\alpha^{-}, \beta\right)$. D'après 1.5(7), il crée le même nombre $\mu_{1}$ et les partitions $\left(\alpha^{\prime}-, \beta^{\prime}\right)$. On obtient de même

$$
S_{c}\left(p_{A, B ; s}\left(\alpha^{-}, \beta,<^{-}\right)\right)=\left\{\begin{array}{cl}
\mu_{1}+B, & \text { si } c=1 \\
\mu_{1}+B+S_{c-1}\left(p_{A, B-s ; s}\left(\alpha^{\prime}, \beta^{\prime},<^{\prime}-\right)\right), & \text { si } c \geq 2 .
\end{array}\right.
$$

Les inégalités à démontrer sont alors claires si $c=1$ et s'obtiennent par récurrence si $c \geq 2$, compte tenu de l'inégalité $\alpha_{1}^{\prime}+\beta_{1}^{\prime} \leq \alpha_{1}+\beta_{1}$.

Supposons maintenant $\alpha_{1}+A>B$. L'ensemble $P_{A, B ; s}^{a}(\alpha, \beta,<)$ n'est pas vide. Le procédé (a) appliqué à $(\alpha, \beta)$ crée un nombre $\nu_{1}$ et des partitions $\left(\alpha^{\prime}, \beta^{\prime}\right)$. Comme ci-dessus, on a

$$
p_{A, B ; s}(\alpha, \beta,<)=\left\{\nu_{1}+A\right\} \sqcup p_{A-s, B ; s}\left(\alpha^{\prime}, \beta^{\prime},<^{\prime}\right),
$$

et $\nu_{1}+A$ est le plus grand terme de $p_{A, B ; s}(\alpha, \beta,<)$.

Supposons $(1,0)<^{-}(1,1)$ et $\alpha_{1}^{-}+A \geq B$. L'ensemble $P_{A, B ; s}^{a}\left(\alpha^{-}, \beta,<^{-}\right)$n'est pas vide. D'après 1.5 (5), le procédé (a) appliqué à $\left(\alpha^{-}, \beta\right)$ crée le nombre $\nu_{1}-\alpha_{1}+\alpha_{2}$ et les partitions $\left(\alpha^{\prime}-, \beta^{\prime}\right)$. On obtient comme ci-dessus

$$
p_{A, B ; s}\left(\alpha^{-}, \beta,<^{-}\right)=\left\{\nu_{1}-\alpha_{1}+\alpha_{2}+A\right\} \sqcup p_{A-s, B ; s}\left(\alpha^{\prime}-, \beta^{\prime},<^{\prime}-\right),
$$

où $\nu_{1}-\alpha_{1}+\alpha_{2}+A$ est le plus grand terme de $p_{A, B ; s}\left(\alpha^{-}, \beta,<^{-}\right)$. En comparant avec (1), la première inégalité de l'énoncé s'en déduit, par récurrence si $c \geq 2$. La seconde est aussi claire si $c=1$. Si $c \geq 2$, on a par récurrence

$$
\begin{gathered}
S_{c}\left(p_{A, B ; s}\left(\alpha^{-}, \beta,<^{-}\right)\right)=\nu_{1}+A+S_{c-1}\left(p_{A-s, B ; s}\left(\alpha^{\prime}, \beta^{\prime},<^{\prime}\right)\right) \\
\leq \nu_{1}+A+S_{c-1}\left(p_{A-s, B ; s}\left(\alpha^{\prime}, \beta^{\prime},<^{\prime}\right)\right)+\alpha_{1}^{\prime}+\beta_{1}^{\prime} \\
\leq S_{c}\left(p_{A, B ; s}\left(\alpha^{-}, \beta,<^{-}\right)\right)+\alpha_{1}-\alpha_{2}+\alpha_{1}^{\prime}+\beta_{1}^{\prime} .
\end{gathered}
$$

Mais $\alpha_{1}^{\prime} \leq \alpha_{2}$ et $\beta_{1}^{\prime} \leq \beta_{1}$, d'où l'inégalité cherchée.

Supposons $(1,0)<^{-}(1,1)$ et $\alpha_{1}^{-}+A<B$. Alors l'ensemble $P_{A, B ; s}^{b}\left(\alpha^{-}, \beta,<^{-}\right)$ n'est pas vide. D'après 1.5 (4), le procédé (b) appliqué à $\left(\alpha^{-}, \beta\right)$ crée le nombre $\nu_{1}-\alpha_{1}$ et les mêmes partitions $\left(\alpha^{\prime}, \beta^{\prime}\right)$. On obtient comme ci-dessus

$$
p_{A, B ; s}\left(\alpha^{-}, \beta,<^{-}\right)=\left\{\nu_{1}-\alpha_{1}+B\right\} \sqcup p_{A, B-s ; s}\left(\alpha^{\prime}, \beta^{\prime},<^{\prime}\right),
$$

où $\nu_{1}-\alpha_{1}+B$ est le plus grand terme de $p_{A, B ; s}\left(\alpha^{-}, \beta,<^{-}\right)$. On compare avec (1). Si $c=1$ on doit prouver que $\nu_{1}-\alpha_{1}+B \leq \nu_{1}+A \leq \nu_{1}-\alpha_{1}+B+\alpha_{1}+\beta_{1}=\nu_{1}+\beta_{1}+B$. La première inégalité résulte de l'hypothèse $\alpha_{1}+A>B$. La seconde résulte de l'hypothèse $\alpha_{1}^{-}+A<B$ qui implique $A<B$, a fortiori $A<B+\beta_{1}$. Supposons $c \geq 2$. Le lemme 2.6 et son symétrique nous disent que

$$
\begin{aligned}
& S_{c-1}\left(p_{A, B-s ; s}\left(\alpha^{\prime}, \beta^{\prime},<^{\prime}\right)\right) \leq S_{c-1}\left(p_{A-s, B ; s}\left(\alpha^{\prime}, \beta^{\prime},<^{\prime}\right)\right)+\sup \left(\alpha_{1}^{\prime}+A-B, 0\right), \\
& S_{c-1}\left(p_{A-s, B ; s}\left(\alpha^{\prime}, \beta^{\prime},<^{\prime}\right)\right) \leq S_{c-1}\left(p_{A, B-s ; s}\left(\alpha^{\prime}, \beta^{\prime},<^{\prime}\right)\right)+\sup \left(\beta_{1}^{\prime}+B-A, 0\right) .
\end{aligned}
$$

D'après l'hypothèse $\alpha_{1}+A>B$ et parce que $\alpha_{1}^{\prime} \leq \alpha_{1}$, on a $\sup \left(\alpha_{1}^{\prime}+A-B, 0\right) \leq$ $\alpha_{1}+A-B$. De même, on vient de voir que $A<B+\beta_{1}$ et on en déduit $\sup \left(\beta_{1}^{\prime}+\right.$ $B-A, 0) \leq \beta_{1}+B-A$. Alors

$$
\begin{gathered}
S_{c}\left(p_{A, B ; s}\left(\alpha^{-}, \beta,<^{-}\right)\right)=\nu_{1}-\alpha_{1}+B+S_{c-1}\left(p_{A, B-s ; s}\left(\alpha^{\prime}, \beta^{\prime},<^{\prime}\right)\right) \\
\leq \nu_{1}-\alpha_{1}+B+S_{c-1}\left(p_{A-s, B ; s}\left(\alpha^{\prime}, \beta^{\prime},<^{\prime}\right)\right)+\alpha_{1}+A-B= \\
\nu_{1}+A+S_{c-1}\left(p_{A-s, B ; s}\left(\alpha^{\prime}, \beta^{\prime},<^{\prime}\right)\right) \\
=S_{c}\left(p_{A, B ; s}(\alpha, \beta,<)\right),
\end{gathered}
$$

et

$$
S_{c}\left(p_{A, B ; s}(\alpha, \beta,<)\right)=\nu_{1}+A+S_{c-1}\left(p_{A-s, B ; s}\left(\alpha^{\prime}, \beta^{\prime},<^{\prime}\right)\right)
$$




$$
\begin{gathered}
\leq \nu_{1}+A+S_{c-1}\left(p_{A, B-s ; s}\left(\alpha^{\prime}, \beta^{\prime},<^{\prime}\right)\right)+\beta_{1}+B-A \\
=\nu_{1}-\alpha_{1}+B+S_{c-1}\left(p_{A, B-s ; s}\left(\alpha^{\prime}, \beta^{\prime},<^{\prime}\right)\right)+\alpha_{1}+\beta_{1} \\
=S_{c}\left(p_{A, B ; s}\left(\alpha^{-}, \beta,<^{-}\right)\right)+\alpha_{1}+\beta_{1} .
\end{gathered}
$$

Ce sont les inégalités cherchées.

Supposons $(1,1)<^{-}(1,0)$ et $\beta_{1}+B \geq A$. Alors $P_{A, B ; s}^{b}\left(\alpha^{-}, \beta,<^{-}\right)$n'est pas vide. On obtient la même relation (2) que dans le cas précédent et on conclut de même (on n'a utilisé l'inégalité $\alpha_{1}^{-}+A<B$ du cas précédent que via son implication $\beta_{1}+B \geq A$, laquelle est ici vraie par hypothèse).

Supposons enfin $(1,1)<^{-}(1,0)$ et $\beta_{1}+B<A$. Alors $P_{A, B ; s}^{a}\left(\alpha^{-}, \beta,<^{-}\right)$n'est pas vide. D'après 1.5(6), le procédé (a) appliqué à $\left(\alpha^{-}, \beta\right)$ crée le nombre $\nu_{1}-\alpha_{1}-\beta_{1}$ les partitions $\left(\underline{\alpha}^{\prime}, \underline{\beta}^{\prime}\right)=\left(\alpha^{\prime},\left\{\beta_{1}\right\} \sqcup \beta^{\prime}\right)$. On obtient

$$
p_{A, B ; s}\left(\alpha^{-}, \beta,<^{-}\right)=\left\{\nu_{1}-\alpha_{1}-\beta_{1}+A\right\} \sqcup p_{A-s, B ; s}\left(\underline{\alpha}^{\prime}, \underline{\beta}^{\prime}, \leq^{\prime}\right)
$$

et $\nu_{1}-\alpha_{1}-\beta_{1}+A$ est le plus grand terme de $p_{A, B ; s}\left(\alpha^{-}, \beta,<^{-}\right)$. En comparant avec (1), le lemme en résulte immédiatement si $c=1$. Si $c \geq 2$, on remarque que $\left(\alpha^{\prime}, \beta^{\prime}\right)=\left(\underline{\alpha}^{\prime}, \underline{\beta}^{\prime}\right)$. On peut appliquer le symétrique de notre lemme à $\left(\underline{\alpha}^{\prime}, \underline{\beta}^{\prime}\right)$. On obtient

$$
\begin{gathered}
S_{c-1}\left(p_{A-s, B ; s}\left(\alpha^{\prime}, \beta^{\prime},<^{\prime}\right)\right) \leq S_{c-1}\left(p_{A-s, B ; s}\left(\underline{\alpha}^{\prime}, \underline{\beta}^{\prime}, \leq^{\prime}\right)\right) \\
\leq S_{c-1}\left(p_{A-s, B ; s}\left(\alpha^{\prime}, \beta^{\prime},<^{\prime}\right)\right)+\alpha_{1}^{\prime}+\beta_{1}^{\prime} .
\end{gathered}
$$

D'où

$$
\begin{gathered}
S_{c}\left(p_{A, B ; s}\left(\alpha^{-}, \beta,<^{-}\right)\right)=\nu_{1}-\alpha_{1}-\beta_{1}+A+S_{c-1}\left(p_{A-s, B ; s}\left(\underline{\alpha}^{\prime}, \underline{\beta}^{\prime}, \leq^{\prime}\right)\right) \\
\leq \nu_{1}-\alpha_{1}-\beta_{1}+A+S_{c-1}\left(p_{A-s, B ; s}\left(\alpha^{\prime}, \beta^{\prime},<^{\prime}\right)\right)+\alpha_{1}^{\prime}+\beta_{1}^{\prime} \\
\leq \nu_{1}+A+S_{c-1}\left(p_{A-s, B ; s}\left(\alpha^{\prime}, \beta^{\prime},<^{\prime}\right)\right) \\
=S_{c}\left(p_{A, B ; s}(\alpha, \beta,<)\right),
\end{gathered}
$$

car $\alpha_{1}^{\prime} \leq \alpha_{1}$ et $\beta_{1}^{\prime} \leq \beta_{1}$. On a aussi

$$
\begin{gathered}
S_{c}\left(p_{A, B ; s}(\alpha, \beta,<)\right)=\nu_{1}+A+S_{c-1}\left(p_{A-s, B ; s}\left(\alpha^{\prime}, \beta^{\prime},<^{\prime}\right)\right) \\
\leq \nu_{1}+A+S_{c-1}\left(p_{A-s, B ; s}\left(\underline{\alpha}^{\prime}, \underline{\beta}^{\prime}, \leq^{\prime}\right)\right) \\
=S_{c}\left(p_{A, B ; s}\left(\alpha^{-}, \beta,<^{-}\right)\right)+\alpha_{1}+\beta_{1} .
\end{gathered}
$$

Cela achève la preuve du lemme 2.5.

2.8. Preuve du lemme 2.6. Supposons d'abord $n_{<}=0$ ou $m_{<}=0$. Alors $p_{A, B-s ; s}(\alpha, \beta,<)=\Lambda_{A, B-s ; s}(\alpha, \beta)$ et $p_{A-s, B ; s}(\alpha, \beta,<)=\Lambda_{A-s, B ; s}(\alpha, \beta)$. Soient $a, b \in \mathbb{N}$ tels que $a+b=c$ et

$$
S_{c}\left(\Lambda_{A, B-s ; s}(\alpha, \beta)\right)=S_{a}\left(\alpha+\left[A,-\infty[s)+S_{b}(\beta+[B-s,-\infty[s) .\right.\right.
$$

Si $a=0$, on a $b=c$ et $S_{c}\left(\beta+\left[B-s,-\infty[s) \leq S_{c}\left(\beta+\left[B,-\infty[s) \leq S_{c}\left(\Lambda_{A-s, B ; s}(\alpha, \beta)\right)\right.\right.\right.\right.$, d'où $S_{c}\left(\Lambda_{A, B-s ; s}(\alpha, \beta)\right) \leq S_{c}\left(\Lambda_{A-s, B ; s}(\alpha, \beta)\right)$. Si au contraire $a \geq 1$, on vérifie que

$$
\begin{gathered}
S_{a}\left(\alpha+\left[A,-\infty\left[{ }_{s}\right)=S_{a-1}\left(\alpha+\left[A-s,-\infty[s)+\alpha_{a}+A,\right.\right.\right.\right. \\
S_{b}\left(\beta+\left[B-s,-\infty[s)=S_{b+1}\left(\beta+\left[B,-\infty[s)-\beta_{b+1}-B .\right.\right.\right.\right.
\end{gathered}
$$

D'où

$$
\begin{aligned}
S_{c}\left(\Lambda_{A, B-s ; s}(\alpha, \beta)\right)= & S_{a-1}\left(\alpha+\left[A-s,-\infty[s)+S_{b+1}\left(\beta+\left[B,-\infty\left[{ }_{s}\right)+\alpha_{a}-\beta_{b+1}+A-B\right.\right.\right.\right. \\
& \leq S_{c}\left(\Lambda_{A-s, B ; s}(\alpha, \beta)\right)+\alpha_{1}+A-B .
\end{aligned}
$$

Supposons désormais $n_{<}>0$ et $m_{<}>0$. Supposons d'abord que des éléments de $P_{A, B-s ; s}(\alpha, \beta,<)$ et $P_{A-s, B ; s}(\alpha, \beta,<)$ puissent se construire par le même procédé 
(a), resp. (b). Ce procédé crée un nombre $\nu_{1}$, resp. $\mu_{1}$, et des partitions $\left(\alpha^{\prime}, \beta^{\prime}\right)$. Par un calcul déjà fait plusieurs fois, on obtient

$$
\left\{\begin{array}{c}
p_{A, B-s ; s}(\alpha, \beta,<)=\left\{\nu_{1}+A\right\} \sqcup p_{A-s, B-s ; s}\left(\alpha^{\prime}, \beta^{\prime},<^{\prime}\right), \\
p_{A-s, B ; s}(\alpha, \beta,<)=\left\{\nu_{1}+A-s\right\} \sqcup p_{A-2 s, B ; s}\left(\alpha^{\prime}, \beta^{\prime},<^{\prime}\right),
\end{array}\right.
$$

resp.

$$
\left\{\begin{array}{c}
p_{A, B-s ; s}(\alpha, \beta,<)=\left\{\mu_{1}+B-s\right\} \sqcup p_{A, B-2 s ; s}\left(\alpha^{\prime}, \beta^{\prime},<^{\prime}\right), \\
p_{A-s, B ; s}(\alpha, \beta,<)=\left\{\mu_{1}+B\right\} \sqcup p_{A-s, B-s ; s}\left(\alpha^{\prime}, \beta^{\prime},<^{\prime}\right) .
\end{array}\right.
$$

Les premiers termes $\nu_{1}+A$ etc... sont les plus grands termes des suites en question. Si $c \geq 2$, on utilise notre lemme par récurrence et on obtient

$$
\begin{gathered}
S_{c}\left(p_{A, B-s ; s}(\alpha, \beta,<)\right) \leq s+\sup \left(\alpha_{1}^{\prime}+A-s-B, 0\right)+S_{c}\left(p_{A-s, B ; s}(\alpha, \beta,<)\right) \\
\leq \sup \left(\alpha_{1}+A-B, s\right)+S_{c}\left(p_{A-s, B ; s}(\alpha, \beta,<)\right),
\end{gathered}
$$

resp.

$$
\begin{gathered}
S_{c}\left(p_{A, B-s ; s}(\alpha, \beta,<)\right) \leq-s+\sup \left(\alpha_{1}^{\prime}+A-B+s, 0\right)+S_{c}\left(p_{A-s, B ; s}(\alpha, \beta,<)\right) \\
\leq \sup \left(\alpha_{1}+A-B,-s\right)+S_{c}\left(p_{A-s, B ; s}(\alpha, \beta,<)\right) .
\end{gathered}
$$

Les majorations ci-dessus sont aussi vraies si $c=1$ : elles résultent immédiatement de (1) et (2). Dans le cas du procédé (b), l'assertion du lemme est alors immédiate. Dans le cas du procédé (a), il reste à voir que $s \leq \alpha_{1}+A-B$. Or, dans ce cas, on a supposé que le procédé (a) était loisible pour construire des éléments de $P_{A-s, B ; s}(\alpha, \beta,<)$. Si $(1,0)<(1,1)$, cela impose directement l'inégalité voulue. Si $(1,1)<(1,0)$, cela impose $\beta_{1}+B \leq A-s$. Mais alors $\alpha_{1}+A-B \geq A-B-\beta_{1} \geq s$ comme on le voulait.

Supposons maintenant que les éléments de $P_{A, B-s ; s}(\alpha, \beta,<)$ et $P_{A-s, B ; s}(\alpha, \beta,<)$ ne puissent pas se construire par le même procédé. On voit que cela impose l'une des conditions suivantes :

(3) $(1,0)<(1,1)$ et $B-s<\alpha_{1}+A<B+s$;

(4) $(1,1)<(1,0)$ et $A-s<\beta_{1}+B<A+s$.

On voit que les éléments de $P_{A, B-s ; s}(\alpha, \beta,<)$ se construisent à l'aide du procédé (a) tandis que ceux de $P_{A-s, B ; s}(\alpha, \beta,<)$ se construisent à l'aide du procédé (b). Le procédé (a), resp. (b), crée un nombre $\nu_{1}$, resp. $\mu_{1}$, et des partitions $\left(\alpha^{\prime}, \beta^{\prime}\right)$, resp. $\left(\underline{\alpha}^{\prime}, \underline{\beta}^{\prime}\right)$. On obtient comme plus haut

$$
\begin{aligned}
& p_{A, B-s ; s}(\alpha, \beta,<)=\left\{\nu_{1}+A\right\} \sqcup p_{A-s, B-s ; s}\left(\alpha^{\prime}, \beta^{\prime},<^{\prime}\right), \\
& p_{A-s, B ; s}(\alpha, \beta,<)=\left\{\mu_{1}+B\right\} \sqcup p_{A-s, B-s ; s}\left(\underline{\alpha}^{\prime}, \underline{\beta}^{\prime}, \leq^{\prime}\right) .
\end{aligned}
$$

Supposons (3) vérifiée. D'après $1.3(10)$, on a $\nu_{1}=\alpha_{1}+\mu_{1}$ et $\left(\underline{\alpha}^{\prime}, \beta^{\prime}\right)=\left(\left\{\alpha_{1}\right\} \sqcup \alpha^{\prime}, \beta^{\prime}\right)$. L'indice $(1,0)$ reste le plus petit indice pour l'ordre $\leq^{\prime}$. Si $c=\overline{1}$, on obtient

$$
S_{1}\left(p_{A, B-s ; s}(\alpha, \beta,<)\right)=\alpha_{1}+A-B+S_{1}\left(p_{A-s, B ; s}(\alpha, \beta,<)\right),
$$

et la majoration cherchée est claire. Si $c \geq 2$, on applique le lemme 2.5 à $\left(\underline{\alpha}^{\prime}, \underline{\beta}^{\prime}\right)$. On obtient

$$
\begin{gathered}
S_{c}\left(p_{A, B-s ; s}(\alpha, \beta,<)\right)=\nu_{1}+A+S_{c-1}\left(p_{A-s, B-s ; s}\left(\underline{\alpha}^{\prime}-, \underline{\beta}^{\prime}, \leq^{\prime}-\right)\right) \\
\leq \nu_{1}+A+S_{c-1}\left(p_{A-s, B-s ; s}\left(\underline{\alpha}^{\prime}, \underline{\beta}^{\prime}, \leq^{\prime}\right)\right)=\alpha_{1}+A-B+S_{c}\left(p_{A-s, B ; s}(\alpha, \beta,<)\right) .
\end{gathered}
$$

D'où la majoration cherchée. 
Supposons (4) vérifiée. D'après 1.3 (10), on a $\mu_{1}=\beta_{1}+\nu_{1}$ et $\left(\alpha^{\prime}, \beta^{\prime}\right)=\left(\underline{\alpha}^{\prime},\left\{\beta_{1}\right\} \sqcup\right.$ $\left.\underline{\beta}^{\prime}\right)$. L'indice $(1,1)$ reste le plus petit indice pour l'ordre $<^{\prime}$. Si $c=1$, on obtient

$$
S_{1}\left(p_{A, B-s ; s}(\alpha, \beta,<)\right)=-\beta_{1}+A-B+S_{1}\left(p_{A-s, B ; s}(\alpha, \beta,<)\right),
$$

et la majoration cherchée est claire : $-\beta_{1}+A-B \leq \alpha_{1}+A-B \leq \sup \left(\alpha_{1}+A-B, 0\right)$. Si $c \geq 2$, on applique le symétrique du lemme 2.5 à $\left(\alpha^{\prime}, \beta^{\prime}\right)$. Il nous dit que

$$
S_{c-1}\left(p_{A-s, B-s ; s}\left(\alpha^{\prime}, \beta^{\prime},<^{\prime}\right)\right) \leq S_{c-1}\left(p_{A-s, B-s ; s}\left(\underline{\alpha}^{\prime}, \underline{\beta}^{\prime}, \leq^{\prime}\right)\right)+\alpha_{1}^{\prime}+\beta_{1}^{\prime} .
$$

On obtient

$$
\begin{gathered}
S_{c}\left(p_{A, B-s ; s}(\alpha, \beta,<)\right)=\nu_{1}+A+S_{c-1}\left(p_{A-s, B-s ; s}\left(\alpha^{\prime}, \beta^{\prime},<^{\prime}\right)\right) \\
\leq \mu_{1}-\beta_{1}+A+S_{c-1}\left(p_{A-s, B-s ; s}\left(\underline{\alpha}^{\prime}, \underline{\beta}^{\prime}, \leq^{\prime}\right)\right)+\alpha_{1}^{\prime}+\beta_{1}^{\prime} \\
=S_{c}\left(p_{A-s, B ; s}(\alpha, \beta,<)\right)+A-B-\beta_{1}+\alpha_{1}^{\prime}+\beta_{1}^{\prime} .
\end{gathered}
$$

Puisque $\beta_{1}^{\prime} \leq \beta_{1}$ et $\alpha_{1}^{\prime} \leq \alpha_{1}$, on a

$$
A-B-\beta_{1}+\alpha_{1}^{\prime}+\beta_{1}^{\prime} \leq \alpha_{1}+A-B \leq \sup \left(\alpha_{1}+A-B, 0\right) .
$$

Cela achève la preuve.

\subsection{Troisième lemme de majoration .}

Lemme. Supposons $(1,0)<(1,1)$. Soient un entier $c \geq 1$ et un réel $\underline{B} \geq B$. Posons $\underline{b}=b_{A-s, \underline{B} ; s}\left(\alpha^{-}, \beta,<^{-} ; c\right)$ et supposons $\underline{b} \geq 1$. Alors on a l'inégalité

$$
S_{c}\left(p_{A-s, \underline{B} ; s}\left(\alpha^{-}, \beta,<^{-}\right)\right)+\alpha_{1}+A-B-s+\underline{b}(B+s-\underline{B}) \leq S_{c}\left(p_{A, B ; s}(\alpha, \beta,<)\right) \text {. }
$$

Preuve. Soit $(\underline{\nu}, \mu)$ l'élément canonique de type (b) de $P_{A-s, \underline{B} ; s}\left(\alpha^{-}, \beta,<^{-}\right)$. Avec la notation de 2.3. , supposons d'abord que

(1) $\iota_{1, \alpha_{1}}(\underline{\nu}, \mu)$ appartient à $P(\alpha, \beta,<)$.

Posons $(\nu, \bar{\mu})=\iota_{1, \alpha_{1}}(\underline{\nu}, \underline{\mu})$, rappelons que $\nu=\left(\alpha_{1}+\underline{\mu}_{1}, \underline{\nu}_{1}, \ldots\right)$ et $\mu=\underline{\mu}^{-}$. Posons $\underline{a}=c-\underline{b}$. Par définition de $\underline{b}$, on a

$$
S_{c}\left(p_{A-s, \underline{B} ; s}\left(\alpha^{-}, \beta,<^{-}\right)\right)=S_{\underline{a}}\left(\underline{\nu}+\left[A-s,-\infty[s)+S_{\underline{b}}(\underline{\mu}+[\underline{B},-\infty[s) .\right.\right.
$$

On a

$$
S_{\underline{b}}\left(\underline{\mu}+\left[\underline{B},-\infty[s)=S_{\underline{b}}(\underline{\mu}+[B,-\infty[s)+\underline{b}(\underline{B}-B)\right.\right.
$$

et

$$
\begin{gathered}
S_{\underline{b}}\left(\underline{\mu}+\left[B,-\infty[s)=S_{\underline{b}}(\underline{\mu})+S_{\underline{b}}([B,-\infty[s)\right.\right. \\
=\underline{\mu}_{1}+S_{\underline{b}-1}\left(\underline{\mu}^{-}\right)+S_{\underline{b}-1}([B,-\infty[s)+B+s-\underline{b} s \\
=S_{\underline{b}-1}\left(\mu+\left[B,-\infty[s)+\underline{\mu}_{1}+B+s-\underline{b} s .\right.\right.
\end{gathered}
$$

On a aussi

$$
S_{\underline{a}}\left(\underline{\nu}+\left[A-s,-\infty[s)=S_{\underline{a}+1}\left(\nu+\left[A,-\infty[s)-\alpha_{1}-\underline{\mu}_{1}-A .\right.\right.\right.\right.
$$

En rassemblant ces égalités, on obtient

$$
\begin{gathered}
S_{c}\left(p_{A-s, \underline{B} ; s}\left(\alpha^{-}, \beta,<^{-}\right)\right)+\alpha_{1}+A-B-s+\underline{b}(B+s-\underline{B}) \\
=S_{\underline{a}+1}\left(\nu+\left[A,-\infty[s)+S_{\underline{b}-1}(\mu+[B,-\infty[s)\right.\right. \\
\leq S_{c}\left(\Lambda_{A, B ; s}(\nu, \mu)\right) \leq S_{c}\left(p_{A, B ; s}(\alpha, \beta,<)\right) .
\end{gathered}
$$

Cela conclut.

D'après le lemme 2.3, l'hypothèse (1) est vérifiée si $(\underline{\nu}, \underline{\mu})$ appartient à l'ensemble $P^{b[1]}\left(\alpha^{-}, \beta,<^{-}\right)$. C'est le cas si $m_{<}=0$. On peut maintenant supposer $m_{<}>0$, d'où $n_{<}>0$ d'après $1.2(1)$. Supposons $n_{<^{-}}=0$. On a $(\underline{\nu}, \underline{\mu})=(\emptyset, \beta)$ et on voit que 
$\iota_{1, \alpha_{1}}(\emptyset, \beta)$ appartient à $P(\alpha, \beta,<)$ : c'est le couple $\left(\nu^{1}, \mu^{1}\right)$ défini en 1.5 (2). Donc (1) est encore vérifiée dans ce cas. On suppose désormais $n_{<^{-}}>0$. Si $(\underline{\nu}, \underline{\mu})$ appartient à $P^{b}\left(\alpha^{-}, \beta,<^{-}\right)$, de nouveau le lemme 2.3 entraîne que l'hypothèse (1) est vérifiée. On suppose donc $(\underline{\nu}, \underline{\mu}) \in P^{a}\left(\alpha^{-}, \beta,<^{-}\right)$. Par construction de l'élément canonique de type (b), cela entraîne que l'une des conditions suivantes est vérifiée :

(2) $(1,0)<^{-}(1,1)$ et $\alpha_{2}+A-s=\alpha_{1}^{-}+A-s>\underline{B}$;

(3) $(1,1)<^{-}(1,0)$ et $\beta_{1}+\underline{B}<A-s$.

Supposons (2) vérifiée. Cela entraîne $\alpha_{1}+A>B$ et l'ensemble $P^{a}(\alpha, \beta,<)$ n'est pas vide. Le procédé (a) appliqué à $(\alpha, \beta)$ construit un nombre $\nu_{1}$ et des partitions extraites $\left(\alpha^{\prime}, \beta^{\prime}\right)$. Comme on l'a déjà vu, on a

$$
p_{A, B ; s}(\alpha, \beta,<)=\left\{\nu_{1}+A\right\} \sqcup p_{A-s, B ; s}\left(\alpha^{\prime}, \beta^{\prime},<^{\prime}\right),
$$

et $\nu_{1}+A$ est le plus grand terme de $p_{A, B ; s}(\alpha, \beta,<)$. D'après 1.5(5), le procédé (a) appliqué à $\left(\alpha^{-}, \beta\right)$ crée le nombre $\underline{\nu}_{1}=\nu_{1}-\alpha_{1}+\alpha_{2}$ et les partitions $\left(\alpha^{\prime}-\beta^{\prime}\right)$. Notons $\left(\underline{\nu}^{\prime}, \underline{\mu}^{\prime}\right)$ l'élément canonique de type (b) de $P_{A-s, \underline{B} ; s}\left(\alpha^{\prime}-, \beta^{\prime},<^{\prime}-\right)$. On a $(\underline{\nu}, \underline{\mu})=\left(\left\{\underline{\nu}_{1}\right\} \sqcup \underline{\nu}^{\prime}, \underline{\mu}^{\prime}\right)$,

$$
\Lambda_{A-s, \underline{B} ; s}(\underline{\nu}, \underline{\mu})=\left\{\underline{\nu}_{1}+A-s\right\} \sqcup \Lambda_{A-2 s, \underline{B} ; s}\left(\underline{\nu}^{\prime}, \underline{\mu}^{\prime}\right) .
$$

De plus, d'après $1.4(7)$, le nombre $\underline{\nu}_{1}+A-s$ est strictement plus grand que tous les autres termes de cette suite de nombres. Cela entraîne $c \geq 2$ (sinon on voit que $\underline{b}=0$ contrairement à l'hypothèse) et que $b_{A-2 s, \underline{B} ; s}\left(\alpha^{\prime}-, \beta^{\prime},<^{\prime} ; c-1\right)=\underline{b}$. On applique le lemme par récurrence à $\left(\alpha^{\prime}, \beta^{\prime}\right)$. On obtient

$S_{c-1}\left(p_{A-2 s, \underline{B} ; s}\left(\alpha^{\prime}-, \beta^{\prime},<^{\prime}-\right)\right)+\alpha_{2}+A-B-2 s+\underline{b}(B+s-\underline{B}) \leq S_{c-1}\left(p_{A, B ; s}\left(\alpha^{\prime}, \beta^{\prime},<^{\prime}\right)\right)$.

D'après les formules ci-dessus, on a

$$
\begin{gathered}
S_{c}\left(p_{A-s, \underline{B} ; s}\left(\alpha^{-}, \beta,<^{-}\right)\right)=\underline{\nu}_{1}+A-s+S_{c-1}\left(p_{A-2 s, \underline{B} ; s}\left(\alpha^{\prime}-, \beta^{\prime},<^{\prime}-\right)\right) \\
=\nu_{1}-\alpha_{1}+\alpha_{2}+A-s+S_{c-1}\left(p_{A-2 s, \underline{B} ; s}\left(\alpha^{\prime}-, \beta^{\prime},<^{\prime}-\right)\right), \\
S_{c}\left(p_{A, B ; s}(\alpha, \beta,<)\right)=\nu_{1}+A+S_{c-1}\left(p_{A, B ; s}\left(\alpha^{\prime}, \beta^{\prime},<^{\prime}\right) .\right.
\end{gathered}
$$

En utilisant les trois inégalités précédentes, on obtient l'inégalité de l'énoncé.

Supposons maintenant (3) vérifiée. On a $\alpha_{1}+A \geq A>A-s>\beta_{1}+\underline{B} \geq \underline{B} \geq B$ et, de nouveau, l'ensemble $P^{a}(\alpha, \beta,<)$ n'est pas vide. Le procédé (a) appliqué à $(\alpha, \beta)$ construit un nombre $\nu_{1}$ et des partitions extraites $\left(\alpha^{\prime}, \beta^{\prime}\right)$. On a encore la relation (4). D'après 1.5(6), le procédé (a) appliqué à $\left(\alpha^{-}, \beta\right)$ crée le nombre $\underline{\nu}_{1}=$ $\nu_{1}-\alpha_{1}-\beta_{1}$ et les partitions $\left(\underline{\alpha}^{\prime}, \underline{\beta}^{\prime}\right)=\left(\alpha^{\prime},\left\{\beta_{1}\right\} \sqcup \beta^{\prime}\right)$. Notons $\left(\underline{\nu}^{\prime}, \underline{\mu}^{\prime}\right)$ l'élément canonique de type (b) de $P_{A-s, \underline{B} ; s}\left(\underline{\alpha}^{\prime}, \underline{\beta}^{\prime}, \leq^{\prime}\right)$. On a encore la relation (5) et les relations $c \geq 2$ et $b_{A-2 s, \underline{B} ; s}\left(\underline{\alpha}^{\prime}, \underline{\beta}^{\prime} ; c-1\right)=\underline{b}$. Remarquons que l'indice $(1,0)$ est le plus petit indice pour l'ordre $\leq^{\prime}$ et on a $\beta^{\prime}=\underline{\beta}^{\prime}$. Appliquons le symétrique du (i) du lemme 2.3. Il affirme l'existence d'un entier $d \geq 1$ et d'un élément $\left(\nu^{\prime}, \mu^{\prime}\right) \in$ $P\left(\alpha^{\prime}, \beta^{\prime},<^{\prime}\right)$ de sorte que

$$
\begin{aligned}
\underline{\nu}^{\prime} & =\left(\nu_{1}^{\prime}, \ldots \nu_{d-1}^{\prime}, \nu_{d+1}^{\prime}, \ldots\right), \\
\underline{\mu}^{\prime} & =\left(\nu_{d}^{\prime}+\beta_{1}, \mu_{1}^{\prime}, \mu_{2}^{\prime}, \ldots\right) .
\end{aligned}
$$

On reprend le calcul fait plus haut sous l'hypothèse (1). On a

$$
\begin{gathered}
S_{\underline{b}}\left(\underline{\mu}^{\prime}+\left[\underline{B},-\infty[s)=S_{\underline{b}}\left(\underline{\mu}^{\prime}+[B,-\infty[s)+\underline{b}(\underline{B}-B),\right.\right.\right. \\
S_{\underline{b}}\left(\underline{\mu}^{\prime}+\left[B,-\infty[s)=S_{\underline{b}}\left(\underline{\mu^{\prime}}\right)+S_{\underline{b}}([B,-\infty[s)\right.\right. \\
=\nu_{d}^{\prime}+\beta_{1}+S_{\underline{b}-1}\left(\mu^{\prime}\right)+S_{\underline{b}-1}([B,-\infty[s)+B+s-\underline{b} s
\end{gathered}
$$




$$
=\nu_{d}^{\prime}+\beta_{1}+B+s-\underline{b} s+S_{\underline{b}-1}\left(\mu^{\prime}+\left[B,-\infty\left[{ }_{s}\right) .\right.\right.
$$

Notons que $\underline{a} \geq 1$ puisque $\underline{\nu}_{1}+A-s$ est strictement plus grand que les autres termes de (5). On a

$$
\begin{gathered}
S_{\underline{a}-1}\left(\underline{\nu}^{\prime}+\left[A-2 s,-\infty[s)=S_{\underline{a}-1}\left(\underline{\nu}^{\prime}\right)+S_{\underline{a}-1}([A-2 s,-\infty[s)\right.\right. \\
=S_{\underline{a}-1}\left(\underline{\nu}^{\prime}\right)+S_{\underline{a}}[A-s,-\infty[s)-A+s .
\end{gathered}
$$

On a

En tout cas,

$$
S_{\underline{a}-1}\left(\underline{\nu}^{\prime}\right)=\left\{\begin{array}{lll}
S_{\underline{a}}\left(\nu^{\prime}\right)-\nu_{\underline{a}}^{\prime}, & \text { si } \quad \underline{a}<d, \\
S_{\underline{a}}\left(\nu^{\prime}\right)-\nu_{d}^{\prime}, & \text { si } \quad \underline{a} \geq d .
\end{array}\right.
$$

d'où

$$
S_{\underline{a}-1}\left(\underline{\nu}^{\prime}\right) \leq S_{\underline{a}}\left(\nu^{\prime}\right)-\nu_{d}^{\prime}
$$

$$
S_{\underline{a}-1}\left(\underline{\nu}^{\prime}+\left[A-2 s,-\infty\left[{ }_{s}\right) \leq-\nu_{d}^{\prime}-A+s+S_{\underline{a}}\left(\nu^{\prime}+[A-s,-\infty[s) .\right.\right.\right.
$$

En rassemblant ces calculs, on obtient

$$
\begin{gathered}
S_{c-1}\left(p_{A-2 s, \underline{B} ; s}\left(\underline{\alpha}^{\prime}, \underline{\beta}^{\prime}, \leq^{\prime}\right)\right)=S_{\underline{a}-1}\left(\underline{\nu}^{\prime}+\left[A-2 s,-\infty[s)+S_{\underline{b}}\left(\underline{\mu}^{\prime}+[B,-\infty[s)\right.\right.\right. \\
\leq-\nu_{d}^{\prime}-A+s+S_{\underline{a}}\left(\nu^{\prime}+\left[A-s,-\infty[s)+\underline{b}(\underline{B}-B)+\nu_{d}^{\prime}+\beta_{1}+B+s-\underline{b} s+S_{\underline{b}-1}\left(\mu^{\prime}+[B,-\infty[s)\right.\right.\right. \\
\leq \beta_{1}-A+B+2 s+\underline{b}(\underline{B}-s-B)+S_{c-1}\left(\Lambda_{A-s, B ; s}\left(\nu^{\prime}, \mu^{\prime}\right)\right) \\
\leq \beta_{1}-A+B+2 s+\underline{b}(\underline{B}-s-B)+S_{c-1}\left(p_{A-s, B ; s}\left(\alpha^{\prime}, \beta^{\prime},<^{\prime}\right)\right) .
\end{gathered}
$$

En vertu de (4) et (5) et de l'égalité $\underline{\nu}_{1}=\nu_{1}-\alpha_{1}-\beta_{1}$, cela entraîne

$$
\begin{gathered}
S_{c}\left(p_{A-s, \underline{B} ; s}\left(\alpha^{-}, \beta,<^{-}\right)\right)=\nu_{1}-\alpha_{1}-\beta_{1}+A-s+S_{c-1}\left(p_{A-2 s, \underline{B} ; s}\left(\underline{\alpha}^{\prime}, \underline{\beta}^{\prime}, \underline{\leq}^{\prime}\right)\right) \\
\leq \nu_{1}+A+S_{c-1}\left(p_{A-s, B ; s}\left(\alpha^{\prime}, \beta^{\prime},<^{\prime}\right)\right)-\alpha_{1}-A+B+s+\underline{b}(\underline{B}-s-B) \\
=S_{c}\left(p_{A, B,<}(\alpha, \beta,<)\right)-\alpha_{1}-A+B+s+\underline{b}(\underline{B}-s-B) .
\end{gathered}
$$

Mais cette inégalité est celle que l'on voulait démontrer.

2.10. Certains ensembles de couples de partitions. Notons $Q_{s}(\alpha, \beta,<)$ l'ensemble des couples $(\nu, \mu) \in \mathcal{P} \times \mathcal{P}$ tels que, pour tous réels $A, B$, on ait la majoration

$$
\text { (1) } \quad \Lambda_{A, B ; s}(\nu, \mu) \leq p_{A, B ; s}(\alpha, \beta,<) \text {. }
$$

Le lemme 1.6 nous dit que $P(\alpha, \beta,<) \subseteq Q_{s}(\alpha, \beta,<)$.

Soit $(\nu, \mu) \in \mathcal{P} \times \mathcal{P}$. Notons $\mathfrak{R}_{s}(\nu, \mu ; \alpha, \beta,<)$ l'ensemble des quadruplets de suites $\mathfrak{r}=\left((A(c))_{c \geq 1},(B(c))_{c \geq 1},(a(c))_{c \geq 1},(b(c))_{c \geq 1}\right)$, où les $A(c)$ et $B(c)$ sont des réels et les $a(c), b(c)$ sont des entiers positifs ou nuls, vérifiant les propriétés suivantes :

(2) pour tout $c \geq 1$,

$$
S_{c}\left(\Lambda_{A(c), B(c) ; s}(\nu, \mu)\right)=S_{a(c)}\left(\nu+\left[A(c),-\infty[s)+S_{b(c)}(\mu+[B(c),-\infty[s) ;\right.\right.
$$

(3) pour tout $c \geq 1$,

$$
S_{c}\left(\Lambda_{A(c), B(c) ; s}(\nu, \mu)\right)=S_{c}\left(p_{A(c), B(c) ; s}(\alpha, \beta,<)\right) ;
$$

(4) pour tout $c \geq 1$, on a $a(c) \leq a(c+1)$ et, pour tout $n \in \mathbb{N}_{>0}$, il existe $c \geq 1$ tel que $a(c)=n$.

Pour un tel quadruplet, on note $c^{0}(\mathfrak{r})$ le plus petit indice $c$ tel que $a(c)=1$. On note $R_{s}(\alpha, \beta,<)$ l'ensemble des couples $(\nu, \mu) \in \mathcal{P} \times \mathcal{P}$ tels que $\mathfrak{R}_{s}(\nu, \mu ; \alpha, \beta,<) \neq \emptyset$. On a

(5) soient $(\nu, \mu) \in \mathcal{P} \times \mathcal{P}$ et $A, B \in \mathbb{R} ;$ supposons $\Lambda_{A, B ; s}(\nu, \mu)=p_{A, B ; s}(\alpha, \beta,<)$; alors $(\nu, \mu) \in R_{s}(\alpha, \beta,<)$. 
En effet, on pose $A(c)=A$ et $B(c)=B$ pour tout $c$ et on choisit pour $a(c)$ le plus grand entier tel que (2) soit vérifié. On voit alors que (4) est aussi vérifiée.

Fixons $(\nu, \mu) \in \mathcal{P} \times \mathcal{P}$ et un entier $k \geq 1$. Définissons une partition ${ }^{k} \nu^{\prime}$ par

(6) ${ }^{k} \nu_{i}^{\prime}=\nu_{i}+1$ si $i=1, \ldots, k-1,{ }^{k} \nu_{i}^{\prime}=\nu_{i+1}$ si $i \geq k$.

Si $k=1$, on a simplement $\nu^{\prime}=\nu^{-}$. Notons $M\left(\alpha_{1}, \nu, \mu, k\right)$ l'ensemble des partitions $\mu^{\prime}$ telles que

(7) $\mu_{1}^{\prime} \geq \mu_{1} \geq \mu_{2}^{\prime} \geq \mu_{2} \geq \ldots$;

(8) $\alpha_{1}+S\left(\mu^{\prime}\right)-S(\mu)=\nu_{k}+1-k$.

Proposition. Supposons $(1,0)<(1,1)$ et soit $\mu^{\prime} \in M\left(\alpha_{1}, \nu, \mu, k\right)$. Supposons que $\left({ }^{k} \nu^{\prime}, \mu^{\prime}\right)$ appartienne à $Q_{s}\left(\alpha^{-}, \beta,<^{-}\right)$. Les propriétés suivantes sont alors vérifiées.

(i) Le couple $(\nu, \mu)$ appartient à $Q_{s}(\alpha, \beta,<)$.

(ii) Si $(\nu, \mu)$ appartient à $R_{s}(\alpha, \beta,<)$, alors $k=1$ et $\left(\nu^{-}, \mu^{\prime}\right) \in R_{s}\left(\alpha^{-}, \beta,<^{-}\right)$. Fixons $\mathfrak{r} \in \mathcal{R}_{s}(\nu, \mu ; \alpha, \beta,<)$ et notons $c^{0}=c^{0}(\mathfrak{r})$. Alors $\left(\nu^{-}, \mu^{\prime}\right)$ est l'unique couple de partitions tel que $\iota_{c^{0}, \alpha_{1}}\left(\nu^{-}, \mu^{\prime}\right)=(\nu, \mu)$.

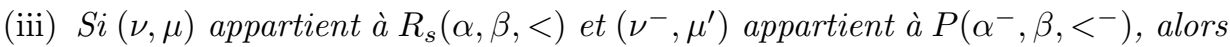
$(\nu, \mu)$ appartient à $P(\alpha, \beta,<)$.

Preuve de (i). Fixons $A$ et $B$ et démontrons l'inégalité (1). Soit $c \in \mathbb{N}_{>0}$, fixons deux entiers $a, b \in \mathbb{N}$ tels que $a+b=c$ et

$$
S_{c}\left(\Lambda_{A, B ; s}(\nu, \mu)\right)=S_{a}\left(\nu+\left[A,-\infty[s)+S_{b}(\mu+[B,-\infty[s) .\right.\right.
$$

Supposons d'abord $a<k$. Alors $\nu_{i}={ }^{k} \nu_{i}^{\prime}-1$ pour tout $i=1, \ldots, a$, donc

$$
S_{a}\left(\nu+\left[A,-\infty[s)=S_{a}\left({ }^{k} \nu^{\prime}+[A,-\infty[s)-a .\right.\right.\right.
$$

D'après (7), on a aussi

$$
S_{b}\left(\mu+\left[B,-\infty[s) \leq S_{b}\left(\mu^{\prime}+\left[B,-\infty\left[{ }_{s}\right) .\right.\right.\right.\right.
$$

Donc

$$
\begin{aligned}
S_{c}\left(\Lambda_{A, B ; s}(\nu, \mu)\right) \leq & S_{a}\left({ }^{k} \nu^{\prime}+\left[A,-\infty\left[{ }_{s}\right)+S_{b}\left(\mu^{\prime}+\left[B,-\infty\left[{ }_{s}\right)-a\right.\right.\right.\right. \\
& \leq S_{c}\left(\Lambda_{A, B ; s}\left({ }^{k} \nu^{\prime}, \mu^{\prime}\right)\right)-a \\
\leq & S_{c}\left(p_{A, B ; s}\left(\alpha^{-}, \beta,<^{-}\right)\right)-a,
\end{aligned}
$$

la dernière inégalité résultant de l'hypothèse $\left({ }^{k} \nu^{\prime}, \mu^{\prime}\right) \in Q_{s}\left(\alpha^{-}, \beta,<^{-}\right)$. On applique le lemme 2.5 et on obtient

$$
S_{c}\left(\Lambda_{A, B ; s}(\nu, \mu)\right) \leq S_{c}\left(p_{A, B ; s}(\alpha, \beta,<)\right)-a \leq S_{c}\left(p_{A, B ; s}(\alpha, \beta,<) .\right.
$$

Supposons maintenant $a \geq k$, a fortiori $a \geq 1$ et $b<c$. On a défini en 2.4 l'entier $b_{A-s, B ; s}\left(\alpha^{-}, \beta,<^{-} ; c\right)$. S'il est supérieur ou égal à $b+1$, on pose $\underline{B}=B$. Sinon, le (ii) du lemme 2.4 dit qu'il existe $\underline{B}>B$ tel que $b_{A-s, B ; s}\left(\alpha^{-}, \beta,<^{-} ; c\right)=b+1$. On fixe un tel $\underline{B}$. Dans les deux cas, on pose $\underline{b}=b_{A-s, \underline{B} ; s}\left(\alpha^{-}, \beta,<^{-} ; c\right)$. On calcule

$$
S_{b}\left(\left[B,-\infty[s)=S_{b+1}([\underline{B},-\infty[s)+(b+1)(B+s-\underline{B})-B-s .\right.\right.
$$

D'où

$$
\begin{gathered}
S_{b}\left(\mu+\left[B,-\infty[s)=S_{b}(\mu)+S_{b}([B,-\infty[s)\right.\right. \\
=S_{b}(\mu)+S_{b+1}([\underline{B},-\infty[s)+(b+1)(B+s-\underline{B})-B-s \\
=S_{b}(\mu)-S_{b+1}\left(\mu^{\prime}\right)+S_{b+1}\left(\mu^{\prime}+[\underline{B},-\infty[s)+(b+1)(B+s-\underline{B})-B-s .\right.
\end{gathered}
$$


Grâce à (6) et à l'hypothèse $a \geq k$, on a

$$
S_{a}(\nu)=\nu_{k}+1-k+S_{a-1}\left({ }^{k} \nu^{\prime}\right) .
$$

D'où

$$
\begin{gathered}
S_{a}\left(\nu+\left[A,-\infty[s)=S_{a}(\nu)+S_{a}([A,-\infty[s)\right.\right. \\
=\nu_{k}+1-k+S_{a-1}\left({ }^{k} \nu^{\prime}\right)+A+S_{a-1}([A-s,-\infty[s) \\
=A+\nu_{k}+1-k+S_{a-1}\left({ }^{k} \nu^{\prime}+[A-s,-\infty[s) .\right.
\end{gathered}
$$

En posant

$$
X=A+\nu_{k}+1-k+S_{b}(\mu)-S_{b+1}\left(\mu^{\prime}\right)+(b+1)(B+s-\underline{B})-B-s,
$$

on obtient

$$
\begin{aligned}
& S_{c}\left(\Lambda_{A, B ; s}(\nu, \mu)\right)=X+S_{a-1}\left({ }^{k} \nu^{\prime}+\left[A-s,-\infty[s)+S_{b+1}\left(\mu^{\prime}+[\underline{B},-\infty[s)\right.\right.\right. \\
& \leq X+S_{c}\left(\Lambda_{A-s, \underline{B} ; s}\left({ }^{k} \nu^{\prime}, \mu^{\prime}\right)\right) \leq X+S_{c}\left(p_{A-s, \underline{B} ; s}\left(\alpha^{-}, \beta,<^{-}\right)\right) .
\end{aligned}
$$

Montrons que

(10) $X \leq \alpha_{1}+A-B-s+\underline{b}(B+s-\underline{B})$.

On écrit

$$
S_{b}(\mu)-S_{b+1}\left(\mu^{\prime}\right)=S(\mu)-S\left(\mu^{\prime}\right)-\mu_{b+1}+\mu_{b+2}^{\prime}-\mu_{b+2}+\mu_{b+3}^{\prime}-\ldots
$$

La série finale est alternée, les valeurs absolues de ses termes étant décroissantes d'après (7). Sa somme est donc négative ou nulle, d'où $S_{b}(\mu)-S_{b+1}\left(\mu^{\prime}\right) \leq S(\mu)-$ $S\left(\mu^{\prime}\right)$. Alors

$$
\begin{gathered}
X \leq A+\nu_{k}+1-k+S(\mu)-S\left(\mu^{\prime}\right)+(b+1)(B+s-\underline{B})-B-s \\
=\alpha_{1}+A-B-s+(b+1)(B+s-\underline{B}) .
\end{gathered}
$$

Pour démontrer (10), il reste à prouver que

$$
(b+1)(B+s-\underline{B}) \leq \underline{b}(B+s-\underline{B}) .
$$

Or, par construction, on a soit $\underline{B}=B$ et $b+1 \leq \underline{b}$, soit $b+1=\underline{b}$. L'inégalité ci-dessus en résulte, d'où aussi (10).

On utilise $(9)$ et $(10)$ et on applique le lemme 2.9 (notons que son hypothèse $\underline{b} \geq 1$ est vérifiée puisque $\underline{b} \geq b+1)$. On obtient

$$
S_{c}\left(\Lambda_{A, B ; s}(\nu, \mu)\right) \leq S_{c}\left(p_{A, B ; s}(\alpha, \beta,<)\right) .
$$

Cela achève de prouver (i).

Preuve de (ii). On fixe un élément $\mathfrak{r}=\left((A(c))_{c \geq 1},(B(c))_{c \geq 1},(a(c))_{c \geq 1},(b(c))_{c \geq 1}\right)$ de $\mathfrak{R}_{s}(\nu, \mu ; \alpha, \beta,<)$. On pose $c^{0}=c^{0}(\mathfrak{r})$. On reprend la démonstration ci-dessus en faisant varier le couple $(A, B)$. Précisément, pour tout $c \geq 1$, on refait les calculs ci-dessus en remplaçant le couple $(A, B)$ par $(A(c), B(c))$ et en choisissant $a=a(c)$ et $b=b(c)$. Cette démonstration prouve l'inégalité

$$
S_{c}\left(\Lambda_{A(c), B(c) ; s}(\nu, \mu)\right) \leq S_{c}\left(p_{A(c), B(c) ; s}(\alpha, \beta,<)\right) .
$$

Or on sait par l'hypothèse (3) que celle-ci est une égalité. Donc toutes les inégalités que l'on a utilisées dans la preuve sont forcément des égalités.

Dans le cas où $a(c)<k$, on a en fait démontré l'inégalité

$$
S_{c}\left(\Lambda_{A(c), B(c) ; s}(\nu, \mu)\right) \leq S_{c}\left(p_{A(c), B(c) ; s}(\alpha, \beta,<)\right)-a(c) .
$$

Donc $a(c)=0$. Puisque, d'après (4), il existe $c$ tel que $a(c)=1$, cela entraîne $k=1$. D'où ${ }^{1} \nu^{\prime}=\nu^{-}$. 
Pour $c<c^{0}$ (ce qui ne se produit que si $c^{0} \geq 2$ ), on a $a(c)=0$ donc $b(c)=c$. On a utilisé l'inégalité

$$
S_{b(c)}\left(\mu+\left[B(c),-\infty[s) \leq S_{b(c)}\left(\mu^{\prime}+[B(c),-\infty[s) .\right.\right.\right.
$$

C'est maintenant une égalité. Avec (7), cela entraîne $\mu_{i}^{\prime}=\mu_{i}$ pour tout $i \leq b(c)=c$, donc pour tout $i<c^{0}$. On a aussi utilisé deux inégalités qui deviennent les égalités

$$
S_{a(c)}\left(\nu^{-}+\left[A(c),-\infty\left[{ }_{s}\right)+S_{b(c)}\left(\mu^{\prime}+\left[B(c),-\infty\left[{ }_{s}\right)=S_{c}\left(\Lambda_{A(c), B(c) ; s}\left(\nu^{-}, \mu^{\prime}\right)\right),\right.\right.\right.\right.
$$

et

$$
S_{c}\left(\Lambda_{A(c), B(c) ; s}\left(\nu^{-}, \mu^{\prime}\right)\right)=S_{c}\left(p_{A(c), B(c) ; s}\left(\alpha^{-}, \beta,<^{-}\right)\right) .
$$

Pour $c \geq c^{0}$, on a utilisé l'inégalité

$$
-\mu_{b(c)+1}+\mu_{b(c)+2}^{\prime}-\mu_{b(c)+2}+\mu_{b(c)+3}^{\prime}-\ldots \leq 0 .
$$

Elle devient une égalité. Grâce à $(7)$, cela entraîne $\mu_{i}^{\prime}=\mu_{i-1}$ pour tout $i \geq b(c)+2$. Dans le cas $c=c^{0}$, on a $a\left(c^{0}\right)=1$ donc $b\left(c^{0}\right)=c^{0}-1$. D'où $\mu_{i}^{\prime}=\mu_{i-1}$ pour tout $i \geq c^{0}+1$. On a vu ci-dessus que $\mu_{i}^{\prime}=\mu_{i}$ pour $i<c^{0}$. Alors tous les termes de $\mu^{\prime}$ sont déterminés sauf $\mu_{c^{0}}^{\prime}$. Mais les égalités précédentes montrent que $\mu_{c^{0}}^{\prime}=S\left(\mu^{\prime}\right)-S(\mu)$. La relation (8) et l'égalité $k=1$ déterminent alors $\mu_{c^{0}}^{\prime}$ : on a $\alpha_{1}+\mu_{c^{0}}^{\prime}=\nu_{1}$. En se reportant à la définition de l'application $\iota_{c^{0}, \alpha_{1}}$, on voit que $(\nu, \mu)=\iota_{c^{0}, \alpha_{1}}\left(\nu^{-}, \mu^{\prime}\right)$. Notons que cette égalité détermine uniquement le couple $\left(\nu^{-}, \mu^{\prime}\right)$.

On a introduit un nombre $\underline{B}$ que l'on note maintenant $\underline{B}(c)$ et utilisé deux inégalités qui deviennent les égalités

$$
\begin{gathered}
S_{a(c)-1}\left(\nu^{-}+\left[A(c)-s,-\infty[s)+S_{b(c)+1}\left(\mu^{\prime}+[\underline{B}(c),-\infty[s)\right.\right.\right. \\
=S_{c}\left(\Lambda_{A(c)-s, \underline{B}(c) ; s}\left(\nu^{-}, \mu^{\prime}\right)\right) \\
S_{c}\left(\Lambda_{A(c)-s, \underline{B}(c) ; s}\left(\nu^{-}, \mu^{\prime}\right)\right)=S_{c}\left(p_{A(c)-s, \underline{B}(c) ; s}\left(\alpha^{-}, \beta,<^{-}\right)\right) .
\end{gathered}
$$

Si $c<c^{0}$, posons $A^{\prime}(c)=A(c), B^{\prime}(c)=B(c), a^{\prime}(c)=a(c)=0, b^{\prime}(c)=b(c)=c$. Si $c \geq c^{0}$, posons $A^{\prime}(c)=A(c)-s, B^{\prime}(c)=\underline{B}(c), a^{\prime}(c)=a(c)-1, b^{\prime}(c)=b(c)+1$. Alors le quadruplet $\mathfrak{r}^{\prime}=\left(\left(A^{\prime}(c)\right)_{c \geq 1},\left(B^{\prime}(c)\right)_{c \geq 1},\left(a^{\prime}(c)\right)_{c \geq 1},\left(b^{\prime}(c)\right)_{c \geq 1}\right)$ appartient à $\mathfrak{R}_{s}\left(\nu^{-}, \mu^{\prime} ; \alpha^{-}, \beta,<^{-}\right)$. En effet, l'analogue de (2) résulte de (11) et (13), l'analogue de (3) résulte de (12) et (14) et l'analogue de (4) résulte de la relation (4) initiale et de la définition de la suite $\left(a^{\prime}(c)\right)_{c \geq 1}$. Donc $\left(\nu^{-}, \mu^{\prime}\right)$ appartient à $\mathfrak{R}_{s}\left(\alpha^{-}, \beta,<^{-}\right)$. Cela démontre (ii).

Preuve de (iii). On poursuit la preuve ci-dessus. On a $a^{\prime}(c)=a(c)=0$ pour $c<c^{0}$ et aussi $a^{\prime}\left(c^{0}\right)=a\left(c^{0}\right)-1=0$. Donc $b^{\prime}(c)=c$ pour $c \leq c^{0}$. Les analogues des relations $(2)$ et $(3)$ nous disent alors que

$$
S_{c}\left(\mu^{\prime}+\left[B^{\prime}(c),-\infty[s)=S_{c}\left(p_{A^{\prime}(c), B^{\prime}(c) ; s}\left(\alpha^{-}, \beta,<^{-}\right)\right)\right.\right.
$$

pour tout $c=1, \ldots, c^{0}$. Supposons $\left(\nu^{-}, \mu^{\prime}\right) \in P\left(\alpha^{-}, \beta,<^{-}\right)$. La condition du lemme 2.2 est vérifiée. Ce lemme nous dit que $\left(\nu^{-}, \mu^{\prime}\right)$ appartient à $P^{b\left[c^{0}\right]}\left(\alpha^{-}, \beta,<^{-}\right)$. Le (ii) du lemme 2.3 implique alors que $(\nu, \mu)$ appartient à $P(\alpha, \beta,<)$. Cela prouve (iii). 
2.11. L'ordre $<_{\alpha, \beta, k}$. On dit qu'un élément de $\mathcal{R}$ est sans multiplicités si c'est une suite strictement décroissante. Soit $k \in \mathbb{Z}$. On suppose

(1) l'élément $\Lambda_{k,-k-1 ; 2}(\alpha, \beta)$ de $\mathcal{R}$ est sans multiplicités.

Nous allons définir un ordre sur l'ensemble d'indices de $(\alpha, \beta)$, dont la définition fait intervenir $\alpha, \beta$ et $k$ et que nous noterons $<_{\alpha, \beta, k}$. Pour $x \in \mathbb{R}$, on note $[x]$ sa partie entière. Choisissons un entier $r$ assez grand pour que, en posant $n=r+[k / 2]+1$ et $m=r-[k / 2]$, on ait $n \geq t(\alpha)$ et $m \geq t(\beta)$. On considère que l'ensemble d'indices de $(\alpha, \beta)$ est l'ensemble $I=(\{1, \ldots, n\} \times\{0\}) \cup(\{1, \ldots, m\} \times\{1\})$. Ecrivons $\Lambda_{k,-k-1 ; 2}(\alpha, \beta)=\left(\lambda_{1}, \lambda_{2}, \ldots\right)$. Pour $j \in\{1, \ldots, n\}$, il existe un unique $x_{j} \in \mathbb{N}_{>0}$ tel que le $j$-ième terme de $\alpha+\left[k,-\infty\left[2\right.\right.$ soit égal à $\lambda_{x_{j}}$. Pour $l \in\{1, \ldots, m\}$, il existe un unique $y_{l} \in \mathbb{N}_{>0}$ tel que le $l$-ième terme de $\beta+\left[-k-1,-\infty\left[2\right.\right.$ soit $\lambda_{y_{l}}$. On définit l'ordre de sorte que $(j, 0)<_{\alpha, \beta, k}(l, 1)$ si et seulement si $x_{j}<y_{l}$. Plus concrètement, on voit que $(j, 0)<_{\alpha, \beta, k}(l, 1)$ si et seulement si $\alpha_{j}+k+2-2 j \geq \beta_{l}-k+1-2 l$. Si on remplace l'entier $r$ par $r+1$, l'ensemble d'indices s'accroît des indices $(n+1,0)$ et $(m+1,1)$ et on vérifie que l'on a $(n, 0)<_{\alpha, \beta, k}(m+1,1)<_{\alpha, \beta, k}(n+1,0)$ si $k$ est pair tandis que $(m, 1)<_{\alpha, \beta, k}(n+1,0)<_{\alpha, \beta, k}(m+1,1)$ si $k$ est impair. Il s'ensuit que la classe d'équivalence de l'ordre ainsi défini ne dépend pas du choix de $r$.

Lemme. Soit $k \in \mathbb{Z}$. Supposons l'hypothèse (1) vérifiée. Alors l'ensemble $P_{k,-k-1 ; 2}\left(\alpha, \beta,<_{\alpha, \beta, k}\right)$ a un unique élément.

Preuve. Si $n_{<_{\alpha, \beta, k}}=0$ ou $m_{<_{\alpha, \beta, k}}=0$, c'est trivial puisque $P\left(\alpha, \beta,<_{\alpha, \beta, k}\right)$ n'a qu'un élément. On suppose $n_{<_{\alpha, \beta, k}}>0$ et $m_{<_{\alpha, \beta, k}}>0$. La situation étant symétrique en la transformation $(\alpha, \beta, k) \mapsto(\beta, \alpha,-k-1)$, on ne perd rien à supposer $(1,0)$ $<_{\alpha, \beta, k}(1,1)$. Comme ci-dessus, à l'aide d'un entier $r$, on fixe un représentant de l'ordre, que l'on note simplement $(n, m,<)$. Par définition de cet ordre, l'hypothèse $(1,0)<(1,1)$ signifie que $\alpha_{1}+k>\beta_{1}-1-k$. A fortiori, $\alpha_{1}+k>-1-k$ et seul le procédé (a) est autorisé pour construire les éléments de $P_{k,-1-k ; 2}(\alpha, \beta,<)$. Ce procédé crée un nombre $\nu_{1}$ et un couple de partitions extraites $\left(\alpha^{\prime}, \beta^{\prime}\right)$. Il établit une bijection entre $P_{k,-1-k ; 2}(\alpha, \beta,<)$ et $P_{k-2 ;-1-k ; 2}\left(\alpha^{\prime}, \beta^{\prime},<^{\prime}\right)$. D'après 1.4 (8), ce dernier ensemble est égal à $P_{k-1,-k ; 2}\left(\alpha^{\prime}, \beta^{\prime},<^{\prime}\right)$. Pour montrer qu'il ne contient qu'un élément, il suffit par récurrence de prouver que le triplet $\left(\alpha^{\prime}, \beta^{\prime}, k-1\right)$ vérifie la même hypothèse $(1)$ que $(\alpha, \beta, k)$ et que les ordres $<^{\prime}$ et $<_{\alpha^{\prime}, \beta^{\prime}, k-1}$ sont équivalents. En fait, pour une utilisation future, nous allons prouver un résultat un peu plus fort : on va montrer que, pour $k^{\prime}=k-1$ ou $k^{\prime}=k$, le triplet $\left(\alpha^{\prime}, \beta^{\prime}, k^{\prime}\right)$ vérifie l'hypothèse (1) et que les ordres $<^{\prime}$ et $<_{\alpha^{\prime}, \beta^{\prime}, k^{\prime}}$ sont équivalents.

Rappelons que le procédé (a) crée des suites $a_{1}, a_{2}, \ldots, a_{T}$ et $b_{1}, b_{2}, \ldots, b_{t}$, cf. [1.3. Les derniers indices $T$ et $t$ vérifient soit $t=T$ et $\left(a_{T}, 0\right)<\left(b_{T}, 1\right)$, soit $T=t+1$ et $\left(b_{t}, 1\right)<\left(a_{T}, 0\right)$. Si $r$ est assez grand, ce que l'on suppose, on a $a_{T}=n$ et $b_{t}=m$. On a dit plus haut que $(m, 1)<(n, 0)$ si $k$ est pair tandis que $(n, 0)<(m, 1)$ si $k$ est impair. Il en résulte que $T=t$ si $k$ est impair et $T=t+1$ si $k$ est pair. On pose $n^{\prime}=n-T$ et $m^{\prime}=m-t$. On va prouver les assertions

(2) soient $i \in\left\{1, \ldots, n^{\prime}\right\}$ et $j \in\left\{1, \ldots, m^{\prime}\right\}$; supposons $(i, 0)<^{\prime}(j, 1)$; alors on a l'inégalité $\alpha_{i}^{\prime}+k^{\prime}+2-2 i>\beta_{j}^{\prime}-k^{\prime}+1-2 j$;

(3) soient $i \in\left\{1, \ldots, n^{\prime}\right\}$ et $j \in\left\{1, \ldots, m^{\prime}\right\}$; supposons $(j, 1)<^{\prime}(i, 0)$; alors on a l'inégalité $\beta_{j}^{\prime}-k^{\prime}+1-2 j>\alpha_{i}^{\prime}+k^{\prime}+2-2 i$;

(4) soient $i \in\left\{1, \ldots, n^{\prime}\right\}$ et $j \geq m^{\prime}+1$; alors $\alpha_{i}^{\prime}+k^{\prime}+2-2 i>-k^{\prime}+1-2 j$;

(5) soient $j \in\left\{1, \ldots, m^{\prime}\right\}$ et $i \geq n^{\prime}+1$; alors $\beta_{j}^{\prime}-k^{\prime}+1-2 j>k^{\prime}+2-2 i$.

Montrons d'abord que ces assertions entraînent les propriétés voulues. Soient $i, j \in \mathbb{N}_{>0}$. Si $i \leq n^{\prime}$ ou $j \leq m^{\prime}$, les hypothèses de l'une des assertions ci-dessus sont 
vérifiées. Il en résulte que $\alpha_{i}^{\prime}+k^{\prime}+2-2 i \neq \beta_{j}^{\prime}-k^{\prime}+1-2 j$ (rappelons que, par définition, on a $\alpha_{i}^{\prime}=0$ si $i>n^{\prime}$ et $\beta_{j}^{\prime}=0$ si $j>m^{\prime}$ ). Si $i>n^{\prime}$ et $j>m^{\prime}$, on a aussi $k^{\prime}+2-2 i \neq-k^{\prime}+1-2 j$ car ces entiers ne sont pas de la même parité. Il en résulte que les suites $\alpha^{\prime}+\left[k^{\prime},-\infty\left[{ }_{2}\right.\right.$ et $\beta^{\prime}+\left[-k^{\prime}-1,-\infty\left[_{2}\right.\right.$ n'ont pas d'éléments communs, c'est-à-dire que $\left(\alpha^{\prime}, \beta^{\prime}, k^{\prime}\right)$ vérifie l'hypothèse (1). En fixant un entier $r^{\prime}$ assez grand, on définit alors un représentant $\left(\underline{n}^{\prime}, \underline{m}^{\prime}, \leq^{\prime}\right)$ de l'ordre $<_{\alpha^{\prime}, \beta^{\prime}, k^{\prime}}$. Quitte à accroître $r^{\prime}$, on peut supposer que $n^{\prime} \leq \underline{n}^{\prime}$ et $m^{\prime} \leq \underline{m}^{\prime}$. Montrons que $\left(n^{\prime}, m^{\prime},<^{\prime}\right)$ et $\left(\underline{n}^{\prime}, \underline{m}^{\prime}, \leq^{\prime}\right)$ sont équivalents. Pour cela, on doit prouver les deux assertions suivantes :

(6) soit $i, j \in \mathbb{N}_{>0}$; supposons $\alpha_{i}^{\prime} \neq 0$; alors la condition $j \leq m^{\prime}$ et $(j, 1)<^{\prime}(i, 0)$ est équivalente à la condition $j \leq \underline{m}^{\prime}$ et $(j, 1) \leq \leq^{\prime}(i, 0)$;

(7) soit $i, j \in \mathbb{N}_{>0}$; supposons $\beta_{j}^{\prime} \neq 0$; alors la condition $i \leq n^{\prime}$ et $(i, 0)<^{\prime}(j, 1)$ est équivalente à la condition $i \leq \underline{n}^{\prime}$ et $(i, 0) \leq \leq^{\prime}(j, 1)$.

Prouvons (6). La condition $\alpha_{i}^{\prime} \neq 0$ implique $i \leq n^{\prime} \leq \underline{n}^{\prime}$. Supposons $j \leq m^{\prime}$ et $(j, 1)<^{\prime}(i, 0)$. Alors $j \leq \underline{m}^{\prime}$ puisque $m^{\prime} \leq \underline{m}^{\prime}$. La relation $(3)$ implique $\beta_{j}^{\prime}-k^{\prime}+$ $1-2 j>\alpha_{i}^{\prime}+k^{\prime}+2-2 i$. Par définition de l'ordre $\leq^{\prime}$, cela signifie que $(j, 1) \leq^{\prime}(i, 0)$. Inversement, supposons $j \leq \underline{m}^{\prime}$ et $(j, 1) \leq^{\prime}(i, 0)$. Cela implique $\beta_{j}^{\prime}-k^{\prime}+1-2 j>$ $\alpha_{i}^{\prime}+k^{\prime}+2-2 i$. Jointe à (4), cette inégalité implique $j \leq m^{\prime}$. Jointe à (2), elle implique $(j, 1)<^{\prime}(i, 0)$. Cela prouve $(6)$. La preuve de $(7)$ est analogue.

Le lemme résulte donc bien des relations (2) à (5) que nous allons maintenant prouver. On note $\phi:\left\{1, \ldots, n^{\prime}\right\} \rightarrow\{1, \ldots, n\}-\left\{a_{1}, \ldots, a_{T}\right\}$ et $\psi:\left\{1, \ldots, m^{\prime}\right\} \rightarrow$ $\{1, \ldots, m\}-\left\{b_{1}, \ldots, b_{t}\right\}$ les uniques bijections croissantes. Pour $i \in\left\{1, \ldots, n^{\prime}\right\}$, soit $h(i) \in\{1, \ldots, T\}$ tel que $a_{h(i)}<\phi(i)<a_{h(i)+1}$ (un tel entier existe puisque $a_{1}=1$ et $\left.a_{T}=n\right)$. On vérifie que $\phi(i)=h(i)+i$. La suite $i \mapsto h(i)$ est croissante. De même, pour $j \in\left\{1, \ldots, m^{\prime}\right\}$, soit $g(j) \in\{1, \ldots, t\}$ tel que $b_{g(j)}<\psi(j)<b_{g(j)+1}$ (un tel entier existe car $b_{t}=m$ et $b_{1}=1$ d'après l'hypothèse $\left.(1,0)<(1,1)\right)$. Alors $\psi(j)=j+g(j)$. La suite $j \mapsto g(j)$ est croissante.

Soient $i$ et $j$ comme en (2). Par définition, l'inégalité $(i, 0)<^{\prime}(j, 1)$ équivaut à $(\phi(i), 0)<(\psi(j), 1)$. On a donc $\left(a_{h(i)}, 0\right)<(\phi(i), 0)<(\psi(j), 1)$. Puisque $b_{h(i)}$ est le plus petit entier $l$ tel que $\left(a_{h(i)}, 0\right)<(l, 1)$, on a $b_{h(i)} \leq \psi(j)$ et même $b_{h(i)}<\psi(j)$ puisque $\psi(j)$ n'appartient pas à la suite $\left\{b_{1}, \ldots, b_{t}\right\}$. Si $\left(b_{h(i)}, 1\right)<(\phi(i), 0)$, le même argument montre que $a_{h(i)+1}<\phi(i)$, ce qui contredit la définition de $h(i)$. On a donc $(\phi(i), 0)<\left(b_{h(i)}, 1\right)$. Par définition de l'ordre $<$, cela signifie que

$$
\alpha_{\phi(i)}+k+2-2 \phi(i)>\beta_{b_{h(i)}}-k+1-2 b_{h(i)} .
$$

On a $\alpha_{\phi(i)}=\alpha_{i}^{\prime}$. Puisque $b_{h(i)}<\psi(j)$, on a $\beta_{b_{h(i)}} \geq \beta_{\psi(j)}=\beta_{j}^{\prime}$. De l'inégalité ci-dessus se déduit

$$
\alpha_{i}^{\prime}+k^{\prime}+2-2 i>\beta_{j}^{\prime}-k^{\prime}+1-2 j+2 X
$$

où

$$
X=k^{\prime}-k+j-b_{h(i)}+\phi(i)-i .
$$

Pour prouver $(2)$, il reste à montrer que $X \geq 0$. On a $\phi(i)-i=h(i)$. L'inégalité $b_{h(i)}<\psi(j)$ entraîne que $h(i) \leq g(j)$. La suite $q \mapsto b_{q}$ est strictement croissante. Il en résulte que la suite $q \mapsto q-b_{q}$ est décroissante. Alors

$$
X=k^{\prime}-k+j-b_{h(i)}+h(i) \geq k^{\prime}-k+j-b_{g(j)}+g(j)=k^{\prime}-k+\psi(j)-b_{g(j)} .
$$

Mais $b_{g(j)}<\psi(j)$ par définition, donc $X \geq k^{\prime}-k+1$, d'où $X \geq 0$ puisque $k^{\prime}-k$ vaut 0 ou -1 . Cela prouve (2).

Soient $i$ et $j$ comme en $(3)$. On a cette fois $(\psi(j), 1)<(\phi(i), 0)$. On voit comme ci-dessus que $\left(b_{g(j)}, 1\right)<(\psi(j), 1)<\left(a_{g(j)+1}, 0\right)<(\phi(i), 0)$. Notons que l'égalité 
$b_{t}=m$ entraîne que $g(j)<t$ donc $g(j)+1 \leq T$ et $a_{g(j)+1}$ existe. L'inégalité $(\psi(j), 1)<\left(a_{g(j)+1}, 0\right)$ entraîne

$$
\beta_{\psi(j)}-k+1-2 \psi(j)>\alpha_{a_{g(j)+1}}+k+2-2 a_{g(j)+1} .
$$

Puisque $a_{g(j)+1}<\phi(i)$, on a $h(i) \geq g(j)+1$ et on obtient comme ci-dessus

$$
\beta_{j}^{\prime}-k^{\prime}+1-2 j>\alpha_{i}^{\prime}+k^{\prime}+2-2 i+2 Y,
$$

où

$$
Y=k-k^{\prime}+i-a_{g(j)+1}+\phi(j)-j=k-k^{\prime}+i-a_{g(j)+1}+g(j) .
$$

De nouveau,

$-a_{g(j)+1}+g(j)=-a_{g(j)+1}+(g(j)+1)-1 \geq-a_{h(i)}+h(i)-1=\phi(i)-i-a_{h(i)}-1 \geq-i$.

D'où $Y \geq k-k^{\prime} \geq 0$, ce qui démontre (3).

Soient $i$ et $j$ comme en (4). On a $\alpha_{i}^{\prime}+k^{\prime}+2-2 i \geq k^{\prime}+2-2 n^{\prime}$ et $-k^{\prime}-1-2 m^{\prime} \geq$ $-k^{\prime}+1-2 j$. Il suffit donc de prouver que $k^{\prime}+2-2 n^{\prime}>-k^{\prime}-1-2 m^{\prime}$, ou encore $2 k^{\prime}>2 n^{\prime}-2 m^{\prime}-3$. On a $n^{\prime}-m^{\prime}=n-T-m+t=(r+[k / 2]+1)-(r-[k / 2])+t-T$. Puisque $T=t+1$ si $k$ est pair et $T=t$ si $k$ est impair, on obtient $n^{\prime}-m^{\prime}=k$. L'inégalité $2 k^{\prime}>2 n^{\prime}-2 m^{\prime}-3$ cherchée est alors claire puisque $k^{\prime} \geq k-1$.

Soient $i$ et $j$ comme en (5). Le même argument nous ramène à prouver l'inégalité $2 n^{\prime}-2 m^{\prime}+1>2 k^{\prime}$. On a calculé $n^{\prime}-m^{\prime}=k$ et l'inégalité s'ensuit puisque $k^{\prime} \leq k$. Cela achève la démonstration.

\subsection{Une propriété de l'ordre $<_{\alpha, \beta, k}$ •}

Lemme. Soit $k \in \mathbb{Z}$. On suppose vérifiée l'hypothèse (1) de 2.11. Alors on a l'égalité $P_{k / 2,-(k+1) / 2 ; 1 / 2}\left(\alpha, \beta,<_{\alpha, \beta, k}\right)=P_{k,-1-k ; 2}\left(\alpha, \beta,<_{\alpha, \beta, k}\right)$.

Preuve. On va démontrer l'assertion plus générale

(1) soient $h, k \in \mathbb{Z}$; supposons que $\Lambda_{h,-h-1 ; 2}(\alpha, \beta)$ et $\Lambda_{k,-k-1 ; 2}(\alpha, \beta)$ soient sans multiplicités et que les ordres $<_{\alpha, \beta, h}$ et $<_{\alpha, \beta, k}$ soient équivalents; alors on a les égalités

$$
P_{h / 2,-(k+1) / 2 ; 1 / 2}\left(\alpha, \beta,<_{\alpha, \beta, k}\right)=P_{h,-1-h ; 2}\left(\alpha, \beta,<_{\alpha, \beta, h}\right)=P_{k,-1-k ; 2}\left(\alpha, \beta,<_{\alpha, \beta, k}\right) .
$$

Notons pour simplifier $<$ l'ordre $<_{\alpha, \beta, h}=<_{\alpha, \beta, k}$. L'assertion est claire si $n_{<}=0$ ou $m_{<}=0$ puisqu'alors $P(\alpha, \beta,<)$ n'a qu'un élément. Supposons $n_{<}>0$ et $m_{<}>0$. La situation étant symétrique par la transformation $(\alpha, \beta, h, k) \mapsto(\beta, \alpha,-k-$ $1,-h-1)$, on ne perd rien à supposer $(1,0)<(1,1)$. Le procédé (a) appliqué à $(\alpha, \beta)$ crée un nombre $\nu_{1}$ et un couple de partitions extraites $\left(\alpha^{\prime}, \beta^{\prime}\right)$. Comme dans la preuve précédente, ce procédé crée une bijection entre $P_{h,-h-1 ; 2}(\alpha, \beta,<)$ et $P_{h-1,-h ; 2}\left(\alpha^{\prime}, \beta^{\prime},<^{\prime}\right)$, ainsi qu'entre $P_{k,-k-1 ; 2}(\alpha, \beta,<)$ et $P_{k-1,-k ; 2}\left(\alpha^{\prime}, \beta^{\prime},<^{\prime}\right)$. Montrons que

(2) $P_{h / 2,-(k+1) / 2 ; 1 / 2}(\alpha, \beta,<)=P_{h / 2,-(k+1) / 2 ; 1 / 2}^{a}(\alpha, \beta,<)$.

Puisque $(1,0)<(1,1)$, on doit prouver que $\alpha_{1}+h / 2>-(k+1) / 2$, c'est-à-dire $\alpha_{1}>-(h+k+1) / 2$. C'est clair si $-(h+k+1)<0$ puisque $\alpha_{1} \geq 0$. Supposons $-(h+k+1) \geq 0$. En interprétant $<$ comme l'ordre $<_{\alpha, \beta, h}$, l'hypothèse $(1,0)<(1,1)$ signifie que $\alpha_{1}+h>-h-1$, c'est-à-dire $\alpha_{1}>-2 h-1$. De même, en interprétant $<$ comme l'ordre $<_{\alpha, \beta, k}$, la même hypothèse signifie que $\alpha_{1}>-2 k-1$. D'où $2 \alpha_{1}>$ $-2(h+k+1)$. Puisqu'on a supposé $-(h+k+1) \geq 0$, on a $-2(h+k+1) \geq-(h+k+1)$, d'où $2 \alpha_{1}>-(h+k+1)$ et l'inégalité cherchée $\alpha_{1}>-(h+k+1) / 2$. Cela démontre (2). 
D'après (2), le procédé (a) établit une bijection entre $P_{h / 2,-(k+1) / 2 ; 1 / 2}(\alpha, \beta,<)$ et $P_{(h-1) / 2,-(k+1) / 2 ; 1 / 2}\left(\alpha^{\prime}, \beta^{\prime},<^{\prime}\right)$. Il suffit donc de prouver les égalités

(3) $P_{(h-1) / 2,-(k+1) / 2 ; 1 / 2}\left(\alpha^{\prime}, \beta^{\prime},<^{\prime}\right)=P_{h-1,-h ; 2}\left(\alpha^{\prime}, \beta^{\prime},<^{\prime}\right)=P_{k-1,-k ; 2}\left(\alpha^{\prime}, \beta^{\prime},<^{\prime}\right)$.

En interprétant $<$ comme l'ordre $<_{\alpha, \beta, h}$, on a prouvé dans le paragraphe précédent que $\left(\alpha^{\prime}, \beta^{\prime}, h\right)$ et $\left(\alpha^{\prime}, \beta^{\prime}, h-1\right)$ vérifiaient l'hypothèse 2.11(1) et que les ordres $<^{\prime}$, $<_{\alpha^{\prime}, \beta^{\prime}, h}$ et $<_{\alpha^{\prime}, \beta^{\prime}, h-1}$ étaient équivalents. De même, en interprétant $<$ comme l'ordre $<_{\alpha, \beta, k}$, on a vu que $\left(\alpha^{\prime}, \beta^{\prime}, k\right)$ et $\left(\alpha^{\prime}, \beta^{\prime}, k-1\right)$ vérifiaient l'hypothèse 2.11(1) et que les ordres $<^{\prime},<_{\alpha^{\prime}, \beta^{\prime}, k}$ et $<_{\alpha^{\prime}, \beta^{\prime}, k-1}$ étaient équivalents. On peut donc appliquer notre assertion (1) par récurrence à $\left(\alpha^{\prime}, \beta^{\prime}\right)$ et au couple d'entiers $(h-1, k)$, ainsi qu'au couple d'entiers $(h, k-1)$. Son application au couple $(h-1, k)$ donne directement la première égalité de (3). L'application au couple $(h, k-1)$ fournit l'égalité $P_{h / 2,-k / 2 ; 1 / 2}\left(\alpha^{\prime}, \beta^{\prime},<^{\prime}\right)=P_{k-1,-k ; 2}\left(\alpha^{\prime}, \beta^{\prime},<^{\prime}\right)$. Mais $P_{h / 2,-k / 2 ; 1 / 2}\left(\alpha^{\prime}, \beta^{\prime},<^{\prime}\right)=$ $P_{(h-1) / 2,-(k+1) / 2 ; 1 / 2}\left(\alpha^{\prime}, \beta^{\prime},<^{\prime}\right)$ d'après 1.4(8). Cela donne l'égalité des termes extrêmes de (3). Cela achève la preuve de (3) et de (1).

Le lemme est le cas particulier $h=k$ de (1).

\section{Multiplicités}

3.1. Définition et énoncé de la proposition principale. Pour toute la section, on fixe un couple de partitions $(\alpha, \beta)$ et un ordre $<$ sur leur ensemble d'indices.

Fixons un représentant $(n, m,<)$ de cet ordre. L'ensemble d'indices est alors l'ensemble $I=(\{1, \ldots, n\} \times\{0\}) \cup(\{1, \ldots, m\} \times\{1\})$. Soit $J$ l'ensemble des couples $((i, e),(j, f)) \in I^{2}$ tels que $(i, e)<(j, f)$ et $e \neq f$. Posons $X=\mathbb{N}^{J}$. Un élément de $X$ s'écrit $x=\left(x_{(i, e),(j, f)}\right)_{((i, e),(j, f)) \in J}$, avec des $x_{(i, e),(j, f)} \in \mathbb{N}$. Pour $x \in X$, on définit un couple $(\alpha[x], \beta[x]) \in \mathbb{Z}^{n} \times \mathbb{Z}^{m}$ par

$$
\begin{gathered}
\alpha[x]_{i}=\alpha_{i}+\left(\sum_{j \in\{1, \ldots, m\},(i, 0)<(j, 1)} x_{(i, 0),(j, 1)}\right)-\left(\sum_{j \in\{1, \ldots, m\},(j, 1)<(i, 0)} x_{(j, 1),(i, 0)}\right), \\
\beta[x]_{j}=\beta_{j}+\left(\sum_{i \in\{1, \ldots n\},(j, 1)<(i, 0)} x_{(j, 1),(i, 0)}\right)-\left(\sum_{i \in\{1, \ldots n\},(i, 0)<(j, 1)} x_{(i, 0),(j, 1)}\right) .
\end{gathered}
$$

Pour $(\nu, \mu) \in \mathbb{Z}^{n} \times \mathbb{Z}^{m}$, notons $X(\alpha, \beta,<; \nu, \mu)$ l'ensemble des éléments $x \in X$ tels que $\alpha[x]=\nu$ et $\beta[x]=\mu$.

Remarques. (1) On a $S(\alpha[x])+S(\beta[x])=S(\alpha)+S(\beta)$ pour tout $x \in X$. On peut donc se limiter aux $(\nu, \mu)$ tels que $S(\nu)+S(\mu)=S(\alpha)+S(\beta)$.

(2) L'ensemble $X(\alpha, \beta,<; \nu, \mu)$ est fini. En effet, identifions l'ensemble d'indices $I$ à $\{1, \ldots, n+m\}$ de façon croissante. Nos couples $(\alpha, \beta)$ et $(\alpha[x], \beta[x])$ deviennent des éléments de $\mathbb{Z}^{n+m}$. L'ensemble $X$ devient un ensemble d'éléments $\left(x_{k, l}\right)_{(k, l)}$ où $(k, l)$ parcourt un sous-ensemble $\Sigma$ de celui des couples tels que $k, l \in\{1, \ldots, n+m\}$ et $k<$ l. Pour $\gamma=\left(\gamma_{1}, \ldots, \gamma_{n+m}\right) \in \mathbb{Z}^{n+m}$, posons $<\gamma, \delta>=\sum_{i=1, \ldots, n+m}\left(\frac{n+m+1}{2}-i\right) \gamma_{i}$. On calcule

$$
<(\alpha[x], \beta[x]), \delta>=<(\alpha, \beta), \delta>+\sum_{(k, l) \in \Sigma}(l-k) x_{k, l} .
$$

Tous les coefficients de la dernière somme sont positifs donc, pour un entier $c \in \mathbb{Z}$ quelconque, il n'y a qu'un nombre fini de $x \in X$ tels que $<(\alpha[x], \beta[x]), \delta>=c$. Or un élément de $X(\alpha, \beta,<; \nu, \mu)$ doit vérifier $<(\alpha[x], \beta[x]), \delta>=<(\nu, \mu), \delta>$.

On note $\mathfrak{S}_{n}$ et $\mathfrak{S}_{m}$ les groupes de permutations de $\{1, \ldots, n\}$ et $\{1, \ldots, m\}$. On note $\operatorname{sgn}$ leur caractère signature usuel. Pour $\nu \in \mathbb{Z}^{n}$ et $w \in \mathfrak{S}_{n}$, on définit $\nu^{w}$ et 
$\nu[w]$ par $\nu_{i}^{w}=\nu_{w i}$ et $\nu[w]_{i}=\nu_{w i}+i-w i$. On définit de même $\mu^{v}$ et $\mu[v]$ pour $\mu \in \mathbb{Z}^{m}$ et $v \in \mathfrak{S}_{m}$.

Soient $(\nu, \mu) \in \mathcal{P} \times \mathcal{P}$. Si $t(\nu)>n$ ou $t(\mu)>m$, on pose $\operatorname{mult}(\alpha, \beta,<; \nu, \mu)=0$. Sinon, on considère $(\nu, \mu)$ comme un élément de $\mathbb{Z}^{n} \times \mathbb{Z}^{m}$ et on pose

$$
\operatorname{mult}(\alpha, \beta ; \nu, \mu)=\sum_{w \in \mathfrak{S}_{n}, v \in \mathfrak{S}_{m}} \operatorname{sgn}(w) \operatorname{sgn}(v)|X(\alpha, \beta,<; \nu[w], \mu[v])| .
$$

C'est un entier relatif. Montrons que

(3) cet entier ne dépend pas du représentant de l'ordre $(n, m,<)$ que l'on a choisi.

Preuve. D'après ce que l'on a expliqué en 1.2 , il suffit de montrer qu'il ne change pas si l'on remplace $n$ par $n+1$, resp. $m$ par $m+1$, et que l'on prolonge l'ordre sur $I$ par celui sur le nouvel ensemble d'indices $I^{\prime}$ de sorte que $(n+1,0)$, resp. $(m+1,1)$, soit le plus grand élément de $I^{\prime}$. Contentons-nous de considérer le cas où l'on remplace $n$ par $n+1$. Notons $\left(n+1, m,<^{\prime}\right)$ ce nouveau représentant et affectons d'un ' les objets construits à l'aide de celui-ci. On peut supposer $t(\nu) \leq n+1$ et $t(\mu) \leq m$, sinon les deux multiplicités construites sont nulles. Soient $w^{\prime} \in \mathfrak{S}_{n+1}$ et $v \in \mathfrak{S}_{m}$. Soit $x \in X^{\prime}\left(\alpha, \beta,<^{\prime} ; \nu\left[w^{\prime}\right], \mu[v]\right)$. On a $\alpha[x]_{n+1}=\nu\left[w^{\prime}\right]_{n+1}$. Puisque $(n+1,0)$ est le plus grand élément de l'ordre $<^{\prime}$ et que $\alpha_{n+1}=0$, cette égalité se traduit par

$$
-\sum_{j=1, \ldots, m} x_{(j, 1),(n+1,0)}=\nu_{w^{\prime}(n+1)}+n+1-w^{\prime}(n+1) .
$$

Le membre de gauche est négatif ou nul, celui de droite est positif ou nul. Ils sont donc tous deux nuls. La nullité du membre de droite implique $w^{\prime}(n+1)=$ $n+1$ et $\nu_{n+1}=0$. Si $\nu_{n+1} \neq 0$, c'est-à-dire si $t(\nu)=n+1$, les ensembles $X^{\prime}\left(\alpha, \beta,<^{\prime} ; \nu\left[w^{\prime}\right], \mu[v]\right)$ sont donc tous vides, d'où $\operatorname{mult}\left(\alpha, \beta,<^{\prime} ; \nu, \mu\right)=0=$ $=\operatorname{mult}(\alpha, \beta,<; \nu, \mu)$. Supposons $t(\nu) \leq n$. Dans la somme définissant mult $\left(\alpha, \beta,<^{\prime}\right.$; $\nu, \mu)$, on peut se limiter aux $w^{\prime} \in \mathfrak{S}_{n+1}$ tels que $w^{\prime}(n+1)=n+1$. Un tel $w^{\prime}$ s'identifie par restriction à un élément $w$ de $\mathfrak{S}_{n}$. La nullité du membre de gauche de (4) implique que $x_{(j, 1),(n+1,0)}=0$ pour tout $j$. Alors, en supprimant ces coordonnées nulles, on voit que l'élément $x \in X^{\prime}\left(\alpha, \beta,<^{\prime} ; \nu\left[w^{\prime}\right], \mu[v]\right)$ s'identifie à un élément de $X(\alpha, \beta,<; \nu[w], \mu[v])$. On obtient que $\left|X^{\prime}\left(\alpha, \beta,<^{\prime} ; \nu\left[w^{\prime}\right], \mu[v]\right)\right|=$ $|X(\alpha, \beta,<; \nu[w], \mu[v])|$. L'égalité mult $\left(\alpha, \beta,<^{\prime} ; \nu, \mu\right)=\operatorname{mult}(\alpha, \beta,<; \nu, \mu)$ en résulte.

Proposition. Soient $(\nu, \mu) \in \mathcal{P} \times \mathcal{P}$.

(i) Supposons mult $(\alpha, \beta,<; \nu, \mu) \neq 0$. Alors $(\nu, \mu) \in Q_{s}(\alpha, \beta,<)($ cf. 2.10).

(ii) Soient $A, B \in \mathbb{R}$. Supposons $\Lambda_{A, B ; s}(\nu, \mu)=p_{A, B ; s}(\alpha, \beta,<)$. Alors mult $(\alpha, \beta$, $<; \nu, \mu) \neq 0$ si et seulement si $(\nu, \mu) \in P_{A, B ; s}(\alpha, \beta,<)$.

(iii) Soient $A, B \in \mathbb{R}$. Supposons $(\nu, \mu) \in P_{A, B ; s}(\alpha, \beta,<)$. Alors mult $(\alpha, \beta$, $<; \nu, \mu)=1$.

3.2. Début de la preuve de la proposition. Si $n_{<}=0$ ou $m_{<}=0$, on choisit le représentant minimal de l'ordre. L'ensemble $J$ est alors vide et $X$ est réduit à un élément. On voit que $\operatorname{mult}(\alpha, \beta,<; \nu, \mu)$ vaut 1 si $(\nu, \mu)=(\alpha, \beta)$ et 0 sinon. Puisqu'on a aussi $P(\alpha, \beta,<)=\{(\alpha, \beta)\}$, la proposition est triviale.

On suppose désormais $n_{<}>0$ et $m_{<}>0$ et on raisonne comme toujours par récurrence sur $n_{<}+m_{<}$. On fixe $(\nu, \mu) \in \mathcal{P} \times \mathcal{P}$ et on choisit un représentant 
$(n, m,<)$ de l'ordre tel que $n>t(\nu)$ et $m>t(\mu)$. On ne perd rien à supposer $(1,0)<(1,1)$. On décompose

$$
\operatorname{mult}(\alpha, \beta,<; \nu, \mu)=\sum_{k=1, \ldots, n} \operatorname{mult}_{k}(\alpha, \beta,<; \nu, \mu)
$$

où

$$
\operatorname{mult}_{k}(\alpha, \beta,<; \nu, \mu)=\sum_{w \in \mathfrak{S}_{n}, w 1=k, v \in \mathfrak{S}_{m}} \operatorname{sgn}(w) \operatorname{sgn}(v)|X(\alpha, \beta,<; \nu[w], \mu[v])| .
$$

On fixe $k \in\{1, \ldots, n\}$. On identifie $\mathfrak{S}_{n-1}$ à l'ensemble des $w \in \mathfrak{S}_{n}$ tels que $w 1=k$ : un élément $w^{\prime} \in \mathfrak{S}_{n-1}$ s'identifie à $w$ tel que $w 1=k, w i=w^{\prime}(i-1)$ si $i=2 \ldots, n$ et $w^{\prime}(i-1)<k$, wi $=w^{\prime}(i-1)+1$ si $i=2, \ldots, n$ et $w^{\prime}(i-1) \geq k$. Ainsi, on a $\operatorname{sgn}(w)=(-1)^{k+1} \operatorname{sgn}\left(w^{\prime}\right)$. On note $J^{\prime}$ le sous-ensemble des $((i, e),(j, f)) \in J$ tels que $(i, e) \neq(1,0)$ et $J^{\prime \prime}$ le complémentaire, c'est-à-dire l'ensemble des éléments $((1,0),(j, 1))$, pour $j \in\{1, \ldots, m\}$. L'ensemble $X$ se décompose conformément en $X_{J^{\prime}} \times X_{J^{\prime \prime}}$ et on peut identifier $X_{J^{\prime \prime}}$ à $\mathbb{N}^{m}$. Par l'application $(i, 0) \mapsto(i-1,0), J^{\prime}$ s'identifie à l'ensemble $J^{-}$analogue à $J$ associé à $\left(\alpha^{-}, \beta,<^{-}\right)$et au représentant $\left(n-1, m,<^{-}\right)$de l'ordre $<^{-}$. Alors $X_{J^{\prime}}$ s'identifie de même à l'analogue $X^{-}$de l'ensemble $X$. On écrit tout élément de $X$ sous la forme $\left(x^{\prime},\left(x_{j}\right)_{j=1, \ldots m}\right)$ avec $x^{\prime} \in$ $X^{-}$. Pour $x^{\prime} \in X^{-}$, on définit $\left(\alpha^{-}\left[x^{\prime}\right], \beta\left[x^{\prime}\right]\right)$ de même que l'on a défini $(\alpha[x], \beta[x])$. Pour $x=\left(x^{\prime},\left(x_{j}\right)_{j=1, \ldots, m}\right)$, on calcule

$$
\alpha[x]_{1}=\alpha_{1}+\sum_{j=1, \ldots, m} x_{j}
$$

$\alpha[x]_{i}=\alpha^{-}\left[x^{\prime}\right]_{i-1}$ et $\beta[x]_{j}=\beta\left[x^{\prime}\right]_{j}-x_{j}$ pour $i=2, \ldots, n$ et $j=1, \ldots, m$.

Fixons $w^{\prime} \in \mathfrak{S}_{n-1}$, soit $w \in \mathfrak{S}_{n}$ l'élément associé. Un élément $\left(x^{\prime},\left(x_{j}\right)_{j=1, \ldots m}\right) \in$ $X$ appartient à l'ensemble $X(\alpha, \beta,<; \nu[w], \mu[v])$ si et seulement si on a les égalités suivantes

(2) $\alpha_{1}+\sum_{j=1, \ldots, m} x_{j}=\nu_{k}+1-k$

(3) $\alpha^{-}\left[x^{\prime}\right]_{i-1}=\nu_{w i}-w i+i$ pour $i=2, \ldots, n$;

(4) $\beta\left[x^{\prime}\right]_{j}-x_{j}=\mu_{v j}-v j+j$ pour $j=1, \ldots, m$.

La relation (3) se décompose en $\alpha^{-}\left[x^{\prime}\right]_{i-1}=\nu_{w^{\prime}(i-1)}-w^{\prime}(i-1)+i$ si $w^{\prime}(i-1)<k$ et $\alpha^{-}\left[x^{\prime}\right]_{i-1}=\nu_{w^{\prime}(i-1)+1}-w^{\prime}(i-1)+i-1$ si $w^{\prime}(i-1) \geq k$. Définissons ${ }^{k} \nu^{\prime} \in \mathcal{P}$ par ${ }^{k} \nu_{i}^{\prime}=\nu_{i}+1$ si $i<k$ et ${ }^{k} \nu_{i}^{\prime}=\nu_{i+1}$ si $i \geq k$. La relation (3) équivaut à

(5) $\alpha^{-}\left[x^{\prime}\right]_{i}={ }^{k} \nu_{w^{\prime} i}^{\prime}-w^{\prime} i+i$ pour tout $i \in\{1, \ldots, n-1\}$.

Pour tout élément $y=\left(y_{1}, \ldots, y_{m}\right) \in \mathbb{Z}^{m}$, notons $\operatorname{stab}(y)$ le groupe des $u \in \mathfrak{S}_{m}$ tels que $y^{u}=y$. Notons $\left(\mathbb{Z}^{m}\right)^{+}$l'ensemble des $y$ tels que $y_{1} \geq y_{2} \ldots \geq y_{m}$. Tout élément $y \in \mathbb{Z}^{m}$ s'écrit $y=z^{u}$ pour un unique $z \in\left(\mathbb{Z}^{m}\right)^{+}$et un $u \in \mathfrak{S}_{m}$ dont la classe dans $\operatorname{stab}(z) \backslash \mathfrak{S}_{m}$ est uniquement déterminée. On décompose $X(\alpha, \beta,<$; $\nu[w], \mu[v])$ en réunion

$$
\sqcup_{z, u} X(\alpha, \beta,<; \nu[w], \mu[v], z, u)
$$

où $z$ parcourt $\left(\mathbb{Z}^{m}\right)^{+}$et $u$ parcourt $\operatorname{stab}(z) \backslash \mathfrak{S}_{m}$ et où $X(\alpha, \beta,<; \nu[w], \mu[v], z, u)$ est le sous-ensemble des $x \in X(\alpha, \beta,<; \nu[w], \mu[v])$ tels que

(6) $\beta\left[x^{\prime}\right]_{j}-j=z_{u j}$ pour tout $j=1, \ldots, m$.

L'ensemble $X(\alpha, \beta,<; \nu[w], \mu[v], z, u)$ se décompose en produit d'un sousensemble de $X^{-}$et d'un sous-ensemble de $X_{J^{\prime \prime}}$. Le premier est l'ensemble des éléments $x^{\prime} \in X^{-}$vérifiant les relations (5) et (6). Celles-ci ne dépendent que de $\alpha^{-}, \beta,{ }^{k} \nu^{\prime}\left[w^{\prime}\right], z, u$, notons l'ensemble en question $X^{-}\left(\alpha^{-}, \beta,<^{-} ;{ }^{k} \nu^{\prime}\left[w^{\prime}\right], z, u\right)$. Le 
deuxième ensemble est celui des $\left(x_{j}\right)_{j=1, \ldots, m} \in X_{J^{\prime \prime}}$ vérifiant la relation (2) ainsi que

(7) $z_{u j}-x_{j}=\mu_{v j}-v j$ pour tout $j=1, \ldots, m$.

Remarquons que, si cette dernière relation est vérifiée, on peut remplacer (2) par l'égalité

(8) $\alpha_{1}+S(z)-S(\mu)+\sum_{j=1, \ldots, m} j=\nu_{k}+1-k$.

Celle-ci est indépendante des $x_{j}$. En conséquence, $X(\alpha, \beta,<; \nu[w], \mu[v], z, u)$ est vide si (8) n'est pas vérifiée. Si elle l'est, le sous-ensemble de $X_{J^{\prime \prime}}$ est défini par la seule relation $(7)$ et il est loisible de noter $X_{J^{\prime \prime}}(\mu[v], z, u)$ cet ensemble. Notons $Z\left(\alpha_{1}, \nu_{k}, \mu\right)$ l'ensemble des $z \in\left(\mathbb{Z}^{m}\right)^{+}$vérifiant (8). On obtient

$X(\alpha, \beta,<; \nu[w], \mu[v])=\sqcup_{z, u ; z \in Z\left(\alpha_{1}, \nu_{k}, \mu\right)} X^{-}\left(\alpha^{-}, \beta,<^{-} ;{ }^{k} \nu^{\prime}\left[w^{\prime}\right], z, u\right) \times X_{J^{\prime \prime}}(\mu[v], z, u)$.

Puis

$$
\begin{gathered}
\sum_{v \in \mathfrak{S}_{m}} \operatorname{sgn}(v)|X(\alpha, \beta,<; \nu[w], \mu[v])|=\sum_{\substack{z \in Z\left(\alpha_{1}, \nu_{k}, \mu\right), u \in \operatorname{stab}(z) \backslash \mathfrak{S}_{m}}}\left|X^{-}\left(\alpha^{-}, \beta,<^{-} ;{ }^{k} \nu^{\prime}\left[w^{\prime}\right], z, u\right)\right| \\
\sum_{v \in \mathfrak{S}_{m}} \operatorname{sgn}(v)\left|X_{J^{\prime \prime}}(\mu[v], z, u)\right| .
\end{gathered}
$$

Comme en 2.10, notons $M\left(\alpha_{1}, \nu, \mu, k\right)$ l'ensemble des partitions $\mu^{\prime} \in \mathcal{P}$ vérifiant

$$
\mu_{1}^{\prime} \geq \mu_{1} \geq \mu_{2}^{\prime} \geq \mu_{2} \geq \ldots
$$

et

$$
\alpha_{1}+S\left(\mu^{\prime}\right)-S(\mu)=\nu_{k}+1-k
$$

Notons que (10) entraîne $t\left(\mu^{\prime}\right) \leq t(\mu)+1 \leq m$. Pour $\mu^{\prime} \in M\left(\alpha_{1}, \nu, \mu, k\right)$, notons $z\left(\mu^{\prime}\right) \in \mathbb{Z}^{m}$ l'élément tel que $z\left(\mu^{\prime}\right)_{i}=\mu_{i}^{\prime}-i$. On a $\operatorname{stab}\left(z\left(\mu^{\prime}\right)\right)=\{1\}$. La condition (11) équivaut à (8) pour $z=z\left(\mu^{\prime}\right)$, donc $z\left(\mu^{\prime}\right) \in Z\left(\alpha_{1}, \nu_{k}, \mu\right)$. La condition (6) pour $z=z\left(\mu^{\prime}\right)$ équivaut à $\beta\left[x^{\prime}\right]_{j}=\mu_{u j}^{\prime}-u j+j$. Alors l'ensemble $X^{-}\left(\alpha^{-}, \beta,<^{-}\right.$; $\left.{ }^{k} \nu^{\prime}\left[w^{\prime}\right], z\left(\mu^{\prime}\right), u\right)$ n'est autre que $X^{-}\left(\alpha^{-}, \beta,<^{-},{ }^{k} \nu^{\prime}\left[w^{\prime}\right], \mu^{\prime}[u]\right)$.

Montrons que l'on a

(12) soit $z \in Z\left(\alpha_{1}, \nu_{k}, \mu\right)$; si $\sum_{v \in \mathfrak{S}_{m}} \operatorname{sgn}(v)\left|X_{J^{\prime \prime}}(\mu[v], z, u)\right| \neq 0$, il existe $\mu^{\prime} \in$ $M\left(\alpha_{1}, \nu, \mu, k\right)$ tel que $z=z\left(\mu^{\prime}\right)$; inversement, pour $\mu^{\prime} \in M\left(\alpha_{1}, \nu, \mu, k\right)$, on a

$$
\sum_{v \in \mathfrak{S}_{m}} \operatorname{sgn}(v)\left|X_{J^{\prime \prime}}\left(\mu[v], z\left(\mu^{\prime}\right), u\right)\right|=\operatorname{sgn}(u) .
$$

La suite $\mu_{1}-1, \mu_{2}-2, \ldots$ est strictement décroissante. Puisque $z \in\left(\mathbb{Z}^{m}\right)^{+}$, il existe d'unique entiers $0 \leq l_{1} \leq l_{2} \leq \ldots \leq l_{m} \leq m$ tels que

$$
\begin{gathered}
z_{1} \geq \ldots \geq z_{l_{1}} \geq \mu_{1}-1>z_{l_{1}+1} \geq \ldots \geq z_{l_{2}} \geq \mu_{2}-2>z_{l_{2}+1} \geq \ldots \\
\geq z_{l_{m}} \geq \mu_{m}-m>z_{l_{m}+1} \geq \ldots \geq z_{m} .
\end{gathered}
$$

Soit $v \in \mathfrak{S}_{m}$. La relation (6) se récrit $x_{u v^{-1} h}=z_{u v^{-1} h}-\mu_{h}+h$ pour tout $h=$ $1, \ldots, m$. Puisque les $x_{h}$ sont des entiers positifs ou nuls quelconques, l'ensemble $X_{J^{\prime \prime}}(\mu[v], z, u)$ a au plus un élément et il en a un si et seulement si $z_{u v^{-1} h} \geq \mu_{h}-h$ pour tout $h=1, \ldots, m$, ce qui équivaut à

(13) $u v^{-1} h \leq l_{h}$ pour tout $h=1, \ldots, m$.

Notons $V$ l'ensemble des $v \in \mathfrak{S}_{m}$ qui vérifient cette condition. On obtient

$$
\sum_{v \in \mathfrak{S}_{m}} \operatorname{sgn}(v)\left|X_{J^{\prime \prime}}(\mu[v], z, u)\right|=\sum_{v \in V} \operatorname{sgn}(v) .
$$


Supposons la somme de gauche non nulle. Alors $V$ n'est pas vide. La condition (13) pour $v \in V$ entraîne $u v^{-1} 1, \ldots u v^{-1} h \leq l_{h}$, en particulier $l_{m}=m$. Introduisons le sous-groupe $\mathfrak{T}$ des éléments de $\mathfrak{S}_{m}$ qui conservent chacun des intervalles $\left\{1, \ldots, l_{1}\right\}$, $\left\{l_{1}+1, \ldots, l_{2}\right\} \ldots$. On voit alors que l'action du groupe $u^{-1} \mathfrak{T} u$ par multiplication à droite sur $\mathfrak{S}_{m}$ conserve l'ensemble $V$. En fixant un ensemble $\underline{V}$ de représentants de cette action dans cet ensemble, on obtient

$$
\sum_{v \in V} \operatorname{sgn}(v)=\sum_{v \in \underline{V}} \operatorname{sgn}(v) \sum_{v^{\prime} \in u^{-1} \mathfrak{T} u} \operatorname{sgn}\left(v^{\prime}\right) .
$$

La non-nullité de cette expression entraîne que $\mathfrak{T}=\{1\}$. On en déduit $l_{h}=h$ pour tout $h$. On a alors

$$
z_{1} \geq \mu_{1}-1>z_{2} \geq \mu_{2}-2>\ldots>z_{m} \geq \mu_{m}-m .
$$

En posant $\mu_{i}^{\prime}=z_{i}+i$, on a $\mu^{\prime} \in M\left(\alpha_{1}, \nu, \mu, k\right)$ et $z=z\left(\mu^{\prime}\right)$. Inversement, si $z=z\left(\mu^{\prime}\right)$ pour $\mu^{\prime} \in M\left(\alpha_{1}, \nu, \mu, k\right)$, on a $l_{h}=h$ pour tout $h$. On voit par récurrence sur $h$ que la condition (13) est vérifiée si et seulement si $u v^{-1} h=h$ pour tout $h$, autrement dit $u=v$. On a alors $V=\{u\}$ d'où $\sum_{v \in V} \operatorname{sgn}(v)=\operatorname{sgn}(u)$. Cela démontre (12).

L'égalité (9) et la propriété (12) entraînent

$$
\begin{gathered}
\sum_{w \in \mathfrak{S}_{n}, w 1=k} \sum_{v \in \mathfrak{S}_{m}} \operatorname{sgn}(w) \operatorname{sgn}(v)|X(\alpha, \beta,<; \nu[w], \mu[v])| \\
=(-1)^{k+1} \sum_{\mu^{\prime} \in M\left(\alpha_{1}, \nu, \mu, k\right)} \sum_{w^{\prime} \in \mathfrak{S}_{n-1}} \sum_{v \in \mathfrak{S}_{m}} \operatorname{sgn}\left(w^{\prime}\right) \operatorname{sgn}(v)\left|X\left(\alpha^{-}, \beta,<^{-} ;{ }^{k} \nu^{\prime}\left[w^{\prime}\right], \mu^{\prime}[v]\right)\right| .
\end{gathered}
$$

Autrement dit

$$
\operatorname{mult}_{k}(\alpha, \beta,<; \nu, \mu)=(-1)^{k+1} \sum_{\mu^{\prime} \in M\left(\alpha_{1}, \nu, \mu, k\right)} \operatorname{mult}\left(\alpha^{-}, \beta,<^{-} ;{ }^{k} \nu^{\prime}, \mu^{\prime}\right) .
$$

3.3. Fin de la preuve. Supposons $\operatorname{mult}(\alpha, \beta,<; \nu, \mu) \neq 0$. D'après les égalités (1) et (14) de 3.2. on peut fixer $k \in\{1, \ldots, n\}$ et $\mu^{\prime} \in M\left(\alpha_{1}, \nu, \mu, k\right)$ tels que $\operatorname{mult}\left(\alpha^{-}, \beta,<^{-} ;{ }^{k} \nu^{\prime}, \mu^{\prime}\right) \neq 0$. On applique le (i) de la proposition par récurrence : $\left({ }^{k} \nu^{\prime}, \mu^{\prime}\right)$ appartient à $Q_{s}\left(\alpha^{-}, \beta,<^{-}\right)$. Le (i) de la proposition 2.10 nous dit que $(\nu, \mu)$ appartient à $Q_{s}(\alpha, \beta,<)$, ce qui prouve le (i) de la proposition 3.1 .

Démontrons l'assertion suivante

(1) supposons $(\nu, \mu) \in R_{s}(\alpha, \beta,<)$; alors les conditions suivantes sont équivalentes :

(a) $\operatorname{mult}(\alpha, \beta,<; \nu, \mu)=1$;

(b) $\operatorname{mult}(\alpha, \beta,<; \nu, \mu) \neq 0$;

(c) il existe $k \in\{1, \ldots, n\}$ et $\mu^{\prime} \in M\left(\alpha_{1}, \nu, \mu, k\right)$ tels que $\operatorname{mult}\left(\alpha^{-}, \beta,<^{-} ;{ }^{k} \nu^{\prime}, \mu^{\prime}\right) \neq$ 0 ;

(d) $(\nu, \mu) \in P(\alpha, \beta,<)$.

Evidemment, (a) implique (b). On vient de voir ci-dessus que (b) implique (c). Montrons que (d) implique (c). D'après le (i) du lemme 2.3 si $(\nu, \mu) \in P(\alpha, \beta,<)$, on peut fixer $c \in\{1, \ldots, m\}$ et $\left(\nu^{\prime}, \mu^{\prime}\right) \in P\left(\alpha^{-}, \beta,<^{-}\right)$de sorte que $(\nu, \mu)=$ $\iota_{c, \alpha_{1}}\left(\nu^{\prime}, \mu^{\prime}\right)$. Posons $k=1$. On a $\nu^{\prime}=\nu^{-}={ }^{1} \nu^{\prime}$ et on vérifie que $\mu^{\prime}$ appartient à $M\left(\alpha_{1}, \nu, \mu, 1\right)$. Comme on l'a vu en 2.10, la condition $\left(\nu^{\prime}, \mu^{\prime}\right) \in P\left(\alpha^{-}, \beta,<^{-}\right)$ entraîne que $\left(\nu^{\prime}, \mu^{\prime}\right)$ appartient à $Q_{s}\left(\alpha^{-}, \beta,<^{-}\right)$. Le (ii) de la proposition 2.10 entraîne que $\left(\nu^{\prime}, \mu^{\prime}\right) \in R_{s}(\alpha, \beta,<)$. On applique notre assertion (1) par récurrence : la condition $\left(\nu^{\prime}, \mu^{\prime}\right) \in P\left(\alpha^{-}, \beta,<^{-}\right)$implique mult $\left(\alpha^{-}, \beta,<^{-} ; \nu^{\prime}, \mu^{\prime}\right) \neq 0$. Cela vérifie la condition (c). Supposons maintenant (c) vérifiée et fixons $k$ et $\mu^{\prime}$ comme dans 
cette relation. Comme plus haut, on a $\left({ }^{k} \nu^{\prime}, \mu^{\prime}\right) \in Q_{s}\left(\alpha^{-}, \beta,<^{-}\right)$. Le (ii) de la proposition 2.10 nous dit que $k=1$ donc ${ }^{k} \nu^{\prime}=\nu^{-}$et que la partition $\mu^{\prime}$ est uniquement déterminée. Les sommes (1) et (14) de 3.2 sont réduites à un unique élément et on obtient l'égalité $\operatorname{mult}(\alpha, \beta,<; \nu, \mu)=\operatorname{mult}\left(\alpha^{-} \beta,<^{-} ; \nu^{-}, \mu^{\prime}\right)$. L'assertion (ii) de la proposition 2.10 nous dit aussi que $\left(\nu^{-}, \mu^{\prime}\right)$ appartient à $R_{s}\left(\alpha^{-}, \beta,<^{-}\right)$. Puisque $\operatorname{mult}\left(\alpha^{-}, \beta,<^{-} ; \nu^{-}, \mu^{\prime}\right) \neq 0$, on applique notre assertion par récurrence : on a $\left(\nu^{-}, \mu^{\prime}\right) \in P\left(\alpha^{-}, \beta,<^{-}\right)$et $\operatorname{mult}\left(\alpha^{-}, \beta,<^{-} ; \nu^{-}, \mu^{\prime}\right)=1$. Avec l'égalité que l'on a prouvée ci-dessus, cela entraîne $\operatorname{mult}(\alpha, \beta,<; \nu, \mu)=1$, c'est-à-dire (a). D'autre part, on peut appliquer le (iii) de la proposition 2.10, qui implique que $(\nu, \mu)$ appartient à $P(\alpha, \beta,<)$, c'est-à-dire $(\mathrm{d})$. Cela démontre $(1)$.

Supposons $\Lambda_{A, B ; s}(\nu, \mu)=p_{A, B ; s}(\alpha, \beta,<)$. Donc $(\nu, \mu) \in R_{s}(\alpha, \beta,<)$ d'après 2.10 (5). Alors (b) est équivalent à (c). Mais, sous l'hypothèse $\Lambda_{A, B ; s}(\nu, \mu)=p_{A, B ; s}(\alpha$, $\beta,<)$, les conditions $(\nu, \mu) \in P(\alpha, \beta,<)$ et $P_{A, B ; s}(\alpha, \beta,<)$ sont équivalentes d'après le (ii) du lemme 1.6. Cela démontre le (ii) de la proposition 3.1.

Si $(\nu, \mu) \in P_{A, B ; s}(\alpha, \beta,<)$, on a encore $(\nu, \mu) \in R_{s}(\alpha, \beta,<)$. L'équivalence de (a) et (d) implique la conclusion du (iii) de la proposition 3.1 .

\section{Composantes eXtrémales D'Une Représentation COnStruite PAR LUSZTIG}

4.1. La correspondance de Springer généralisée. Pour $n \in \mathbb{N}$, on note $\mathcal{P}(n)$ l'ensemble des partitions de $n$, c'est-à-dire l'ensemble des partitions $\lambda \in \mathcal{P}$ telles que $S(\lambda)=n$. On note $\mathcal{P}_{2}(n)$ l'ensemble des couples $(\alpha, \beta)$ de partitions telles que $S(\alpha)+S(\beta)=n$. On note $W_{n}$ le groupe de Weyl d'un système de racines de type $C_{n}$, avec la convention $W_{0}=\{1\}$. On note $W_{n}^{\vee}$ l'ensemble des représentations irréductibles de $W_{n}$. Il est bien connu que $W_{n}^{\vee}$ est paramétré par $\mathcal{P}_{2}(n)$. Pour $(\alpha, \beta) \in \mathcal{P}_{2}(n)$, on note $\rho_{\alpha, \beta}$ la représentation de $W_{n}$ paramétrée par ce couple. En particulier, $\rho_{(n), \emptyset}$ est la représentation triviale et $\rho_{\emptyset,\left(1^{n}\right)}$ est le caractère signature usuel, que l'on note $s g n$.

Soit $N \in \mathbb{N}$. Notons $\mathcal{P}^{s y m p}(2 N)$ l'ensemble des partitions symplectiques de $2 N$, c'est-à-dire l'ensemble des partitions $\lambda \in \mathcal{P}(2 N)$ telles que mult $_{\lambda}(i)$ est pair pour tout entier $i$ impair. Pour $\lambda \in \mathcal{P}^{s y m p}(2 N)$, on note $\operatorname{Jord}(\lambda)$ l'ensemble des entiers $i \geq 1$ tels que mult $_{\lambda}(i)>0$ et $\operatorname{Jord}^{b p}(\lambda)$ le sous-ensemble des entiers pairs. On note $\mathcal{P}^{\text {symp }}(2 N)$ l'ensemble des couples $(\lambda, \epsilon)$ où $\lambda \in \mathcal{P}^{\text {symp }}(2 N)$ et $\epsilon \in\{ \pm 1\}^{\operatorname{Jord}^{b p}(\lambda)}$.

La correspondance de Springer généralisée, définie par Springer et Lusztig, établit une bijection entre $\mathcal{P}^{\boldsymbol{s y m p}}(2 N)$ et l'ensemble $\bigcup_{k} W_{N-k(k+1) / 2}^{\vee}$, où $k$ parcourt les éléments de $\mathbb{N}$ tels que $k(k+1) \leq 2 N$. Ou encore avec l'ensemble $\bigcup_{k} \mathcal{P}_{2}(N-k(k+$ 1)/2). Rappelons la définition de cette bijection.

Soit $k \in \mathbb{N}$ tel que $k(k+1) \leq 2 N$ et soit $(\alpha, \beta) \in \mathcal{P}_{2}(N-k(k+1) / 2)$. On définit un couple $\left(A_{\alpha, \beta}, B_{\alpha, \beta}\right)$ d'éléments de $\mathcal{R}$ par

si $k$ est pair, $A_{\alpha, \beta}=\alpha+\left[k,-\infty\left[2, B_{\alpha, \beta}=\beta+[-k-1,-\infty[2\right.\right.$;

si $k$ est impair, $A_{\alpha, \beta}=\beta+\left[-k-1,-\infty\left[2, B_{\alpha, \beta}=\alpha+[k,-\infty[2\right.\right.$.

Soit $(\lambda, \epsilon) \in \mathcal{P}^{\operatorname{symp}}(2 N)$. Considérons l'élément $\lambda+\left[-1,-\infty\left[{ }_{1}\right.\right.$ de $\mathcal{R}$. C'est une suite d'entiers strictement décroissante. On introduit les deux éléments $z=$ $\left(z_{1}, z_{2}, \ldots\right)$ et $z^{\prime}=\left(z_{1}^{\prime}, z_{2}^{\prime}, \ldots\right)$ de $\mathcal{R}$, strictement décroissants, tels que $\lambda+[-1,-\infty[1$ soit la réunion de $\left(2 z_{1}, 2 z_{2}, \ldots\right)$ et de $\left(2 z_{1}^{\prime}+1,2 z_{2}^{\prime}+1, \ldots\right)$. On pose $A_{\lambda}^{\sharp}=z^{\prime}+[1,-\infty[1$, 
$B_{\lambda}^{\sharp}=z+[0,-\infty[1$. On calcule facilement, pour $j \geq 1$,

(1) (a) $\quad A_{\lambda, j}^{\sharp}=\{$

$$
\begin{gathered}
\lambda_{2 j-1} / 2+2-2 j, \\
\left(\lambda_{2 j}-1\right) / 2+2-2 j,
\end{gathered}
$$

si $\lambda_{2 j-1}$ est pair, $\left(\lambda_{2 j-2}+1\right) / 2+2-2 j$,

si $\lambda_{2 j}$ est impair et $S_{2 j}(\lambda)$ est pair,

si $j \geq 2, \lambda_{2 j-2}$ est impair et $S_{2 j-2}(\lambda)$ est impair,

$(1)(b)$

$$
B_{\lambda, j}^{\sharp}=\left\{\begin{array}{ccc}
\lambda_{2 j} / 2+1-2 j, & \text { si } \lambda_{2 j} \text { est pair, } \\
\left(\lambda_{2 j-1}+1\right) / 2+1-2 j, & \text { si } \lambda_{2 j-1} & \text { est impair et } S_{2 j-1}(\lambda) \\
& \text { est impair, } \\
\left(\lambda_{2 j+1}-1\right) / 2+1-2 j, & \text { si } \lambda_{2 j+1} & \text { est impair et } S_{2 j+1}(\lambda) \\
& & \text { est pair. }
\end{array}\right.
$$

On vérifie que les divers cas ci-dessus sont exclusifs l'un de l'autre.

Considérons l'ensemble $\left(A_{\lambda}^{\sharp} \cup B_{\lambda}^{\sharp}\right)-\left(A_{\lambda}^{\sharp} \cap B_{\lambda}^{\sharp}\right)$, c'est-à-dire l'ensemble des entiers relatifs qui interviennent dans $A_{\lambda}^{\sharp}$ ou $B_{\lambda}^{\sharp}$ mais pas dans les deux. Notons $D_{\lambda}$ l'ensemble des sous-ensembles finis de $\left(A_{\lambda}^{\sharp} \cup B_{\lambda}^{\sharp}\right)-\left(A_{\lambda}^{\sharp} \cap B_{\lambda}^{\sharp}\right)$ formés d'entiers consécutifs, et maximaux pour cette propriété. On s'aperçoit qu'il y a une bijection croissante $i \mapsto \Delta_{i}$ de $\operatorname{Jord}^{b p}(\lambda)$ sur $D_{\lambda}$. On pose

$$
\begin{aligned}
& A_{\lambda, \epsilon}=\left(A_{\lambda}^{\sharp}-\bigcup_{i \in J o r d^{b p}(\lambda) ; \epsilon_{i}=-1}\left(A_{\lambda}^{\sharp} \cap \Delta_{i}\right)\right) \cup\left(\bigcup_{i \in J o r d d^{b p}(\lambda) ; \epsilon_{i}=-1}\left(B_{\lambda}^{\sharp} \cap \Delta_{i}\right)\right), \\
& B_{\lambda, \epsilon}=\left(B_{\lambda}^{\sharp}-\bigcup_{i \in J o r d^{b p}(\lambda) ; \epsilon_{i}=-1}\left(B_{\lambda}^{\sharp} \cap \Delta_{i}\right)\right) \cup\left(\bigcup_{i \in J o r d} \bigcup_{J^{b p}(\lambda) ; \epsilon_{i}=-1}\left(A_{\lambda}^{\sharp} \cap \Delta_{i}\right)\right) .
\end{aligned}
$$

On définit l'élément $p_{\lambda, \epsilon}=A_{\lambda, \epsilon} \sqcup B_{\lambda, \epsilon}$ de $\mathcal{R}$. Remarquons que l'on a aussi $p_{\lambda, \epsilon}=$ $A_{\lambda}^{\sharp} \sqcup B_{\lambda}^{\sharp}$.

On montre qu'il existe un unique entier $k \in \mathbb{N}$ tel que $k(k+1) \leq 2 N$ et un unique couple $(\alpha, \beta) \in \mathcal{P}_{2}(N-k(k+1) / 2)$ tels que $\left(A_{\alpha, \beta}, B_{\alpha, \beta}\right)=\left(A_{\lambda, \epsilon}, B_{\lambda, \epsilon}\right)$. La correspondance de Springer généralisée associe à $(\lambda, \epsilon)$ le couple $\left(k, \rho_{\alpha, \beta}\right)$. On note ce couple $(k(\lambda, \epsilon), \rho(\lambda, \epsilon))$.

L'entier $k(\lambda, \epsilon)$ se calcule facilement. Notons $i_{1}>\ldots>i_{m}$ les éléments $i \in$ $J_{o r d}^{b p}(\lambda)$ tels que mult $_{\lambda}(i)$ soit impair. Posons

$$
M(\lambda, \epsilon)=\sum_{l=1, \ldots, m ; \epsilon_{i_{l}}=-1}(-1)^{l} .
$$

Alors $k(\lambda, \epsilon)=2 M(\lambda, \epsilon)$ si $M(\lambda, \epsilon) \geq 0$ et $k(\lambda, \epsilon)=-2 M(\lambda, \epsilon)-1$ si $M(\lambda, \epsilon)<0$.

Considérons le cas particulier où $\operatorname{Jor} d(\lambda)=\operatorname{Jord}^{b p}(\lambda)$, c'est-à-dire que $\lambda$ n'a que des termes pairs. On peut considérer que $\epsilon$ est une fonction définie sur l'ensemble d'indices $\mathbb{N}_{>0}$ : si $j \in \mathbb{N}_{>0}$ est tel que $\lambda_{j} \neq 0$, on pose $\epsilon(j)=\epsilon_{\lambda_{j}}$; si $\lambda_{j}=0$, on pose $\epsilon(j)=1$. On prendra garde de distinguer $\epsilon(j)$ où $j$ est un indice et $\epsilon_{i}$ où $i$ est un terme de $\lambda$. Avec la recette ci-dessus, on calcule

$$
\begin{gathered}
A_{\lambda, \epsilon}=\left\{\lambda_{j} / 2+1-j ; j \in \mathbb{N}_{>0}, \epsilon(j)=(-1)^{j+1}\right\}, \\
B_{\lambda, \epsilon}=\left\{\lambda_{j} / 2+1-j ; j \in \mathbb{N}_{>0}, \epsilon(j)=(-1)^{j}\right\} .
\end{gathered}
$$

On constate que $p_{\lambda, \epsilon}$ est sans multiplicités, cf. 2.11.

Remarquons qu'avec les mêmes hypothèses, on a

(2) $M(\lambda, \epsilon)=\sum_{j \in \mathbb{N}>0}(-1)^{j+1} \frac{\epsilon(j)-1}{2}$. 
Preuve. Remarquons d'abord que seuls les $j \leq t(\lambda)$ contribuent à la somme cidessus : pour $j>t(\lambda)$, on a $\epsilon(j)=1$ et le terme que l'on somme est nul. Soit $i \in \operatorname{Jord}^{b p}(\lambda)$, notons $\left\{j_{i}^{-}, \ldots, j_{i}^{+}\right\}$le sous-ensemble des $j \in \mathbb{N}_{>0}$ tels que $\lambda_{j}=i$. Pour tous ces $j$, on a $\epsilon(j)=\epsilon_{i}$ et la contribution de ces $j$ au membre de droite de (2) est

$$
\frac{\epsilon_{i}-1}{2} \sum_{j=j_{i}^{-}, \ldots, j_{i}^{+}}(-1)^{j+1}=\left\{\begin{array}{cc}
0, & \text { si } j_{i}^{+}-j_{i}^{-} \text {est impair ou si } \epsilon_{i}=1, \\
(-1)^{j_{i}^{-}}, & \text {si } j_{i}^{+}-j_{i}^{-} \text {est pair et } \epsilon_{i}=-1 .
\end{array}\right.
$$

Si $\epsilon_{i}=1$, l'intervalle $\left\{j_{i}^{-}, \ldots, j_{i}^{+}\right\}$ne contribue pas au membre de droite de $(2)$ et l'entier $i$ ne contribue pas non plus à $M(\lambda, \epsilon)$. Supposons $\epsilon_{i}=-1$. On a $j^{+}-$ $j^{-}=$mult $_{\lambda}(i)-1$. Si mult $_{\lambda}(i)$ est pair, l'intervalle $\left\{j_{i}^{-}, \ldots, j_{i}^{+}\right\}$ne contribue pas au membre de droite de (2). L'entier $i$ ne contribue pas non plus à $M(\lambda, \epsilon)$. Si mult $_{\lambda}(i)$ est impair, on a $i=i_{l}$ pour un $l \in\{1, \ldots, m\}$ et on vérifie par récurrence sur $l$ que $j_{i}^{-}$est de la même parité que $l$. Alors l'intervalle $\left\{j_{i}^{-}, \ldots, j_{i}^{+}\right\}$contribue au membre de droite de $(2)$ par $(-1)^{l}$ et $i$ contribue de la même façon à $M(\lambda, \epsilon)$. Cela prouve (2).

4.2. Un résultat de Shoji. Soit $(\lambda, \epsilon) \in \mathcal{P}^{\operatorname{symp}}(2 N)$. On suppose que $\lambda$ n'a que des termes pairs. Notons $k=k(\lambda, \epsilon)$ et $(\alpha, \beta)$ l'élément de $\mathcal{P}_{2}(N-k(k+1) / 2)$ qui correspond à $(\lambda, \epsilon)$ par la correspondance de Springer généralisée. On a vu en 4.1 que la suite $p_{\lambda, \epsilon}$ était sans multiplicités. D'après les définitions, on a l'égalité $p_{\lambda, \epsilon}=\Lambda_{k,-k-1 ; 2}(\alpha, \beta)$. Donc l'hypothèse (1) de 2.11 est vérifiée et l'on définit l'ordre $<_{\alpha, \beta, k}$ sur l'ensemble des indices de $(\alpha, \beta)$.

Soit $\left(\lambda^{\prime}, \epsilon^{\prime}\right) \in \mathcal{P}^{\operatorname{symp}}(2 N)$, supposons $k\left(\lambda^{\prime}, \epsilon^{\prime}\right)=k$. Dans l'introduction, on a défini la multiplicité mult $\left(\lambda, \epsilon ; \lambda^{\prime}, \epsilon^{\prime}\right)$. Notons $\left(\alpha^{\prime}, \beta^{\prime}\right) \in \mathcal{P}_{2}(N-k(k+1) / 2)$ le couple correspondant à $\left(\lambda^{\prime}, \epsilon^{\prime}\right)$.

Proposition. On rappelle que $\lambda$ est à termes pairs. Pour $\left(\lambda^{\prime}, \epsilon^{\prime}\right)$ comme ci-dessus, on a l'égalité

$$
\mathfrak{m u l t}\left(\lambda, \epsilon ; \lambda^{\prime}, \epsilon^{\prime}\right)=\operatorname{mult}\left(\alpha, \beta,<_{\alpha, \beta, k} ; \alpha^{\prime}, \beta^{\prime}\right) .
$$

Shoji démontre ce résultat en [4] p.685. En fait, ses hypothèses sont un peu plus contraignantes que les nôtres aussi allons-nous reprendre sa démonstration. Notre situation est plus simple que celle traitée par Shoji car il considère des familles de partitions $\boldsymbol{\alpha}$ à $e \geq 1$ termes tandis que nous nous limitons aux paires de partitions, c'est-à-dire au cas $e=2$. Nous continuerons donc à noter nos familles comme des paires et n'utiliserons pas la notation $\boldsymbol{\alpha}$ de Shoji. De plus, les exposants \pm qui interviennent parfois dans [4] sont inutiles dans le cas $e=2$ et nous les faisons disparaître.

Les définitions de Shoji ne sont pas tout-à-fait les mêmes que les nôtres. Dans les deux paragraphes suivants, nous adopterons le point de vue de Shoji. Introduisons les notations requises. A l'aide d'un entier $r$ assez grand, on construit comme en 2.11 un représentant de l'ordre $<_{\alpha, \beta, k}$, que l'on note simplement $(n, m,<)$. On note $I$ l'ensemble d'indices pour ce représentant et $\nu_{0}$ le plus grand des deux éléments $(t(\alpha), 0)$ et $(t(\beta), 1) \quad\left(\nu_{0}\right.$ est l'indice $\left(t_{<}, e_{<}\right)$de 1.2). Pour $h \in \mathbb{N}$, on note $\mathcal{P}_{h}$ l'ensemble des $\alpha \in \mathcal{P}$ telles que $t(\alpha) \leq h$. Pour $\left(\alpha^{\prime}, \beta^{\prime}\right) \in \mathcal{P}_{n} \times \mathcal{P}_{m}$, on pose $\boldsymbol{\Lambda}\left(\alpha^{\prime}, \beta^{\prime}\right)=\left(\alpha^{\prime}+\left\{2 r+k, 2 r+k-2, \ldots, \xi^{a}\right\}, \beta^{\prime}+\left\{2 r-k-1,2 r-k-3, \ldots, \xi^{b}\right\}\right)$, où $\left(\xi^{a}, \xi^{b}\right)=(0,1)$ si $k$ est pair et $\left(\xi^{a}, \xi^{b}\right)=(1,0)$ si $k$ est impair. On pose $\Lambda\left(\alpha^{\prime}, \beta^{\prime}\right)=\left(\alpha^{\prime}+\left\{2 r+k, 2 r+k-2, \ldots, \xi^{a}\right\}\right) \sqcup\left(\beta^{\prime}+\left\{2 r-k-1,2 r-k-3, \ldots, \xi^{b}\right\}\right)$. 
Cet élément de $\mathcal{R}_{f}$ est l'ensemble des $2 r+1$ plus grand termes de $\Lambda_{k,-k-1 ; 2}\left(\alpha^{\prime}, \beta^{\prime}\right)$, auxquels on a ajouté $2 r$ pour qu'ils soient tous positifs ou nuls. On pose simplement $\boldsymbol{\Lambda}=\boldsymbol{\Lambda}(\alpha, \beta)$ et $\Lambda=\Lambda(\alpha, \beta)$. La suite $\Lambda$ est formée des $2 r+1$ plus grands termes de $\Lambda_{k,-k-1 ; 2}(\alpha, \beta)=p_{\lambda, \epsilon}$ auxquels on a ajouté $2 r$. Comme $p_{\lambda, \epsilon}$, cette suite est sans multiplicités.

4.3. Démonstration de la proposition 4.2, première étape . La démonstration de Shoji s'applique pourvu que sa proposition 6.1 soit vérifiée. C'est-à-dire qu'avec ses notations, on doit prouver l'égalité $Q_{\Lambda}=R_{\Lambda}$. Ainsi que Shoji l'explique, il suffit de prouver l'assertion suivante. Ecrivons $R_{\boldsymbol{\Lambda}}$ comme combinaison linéaire de fonctions de Schur $s_{\alpha^{\prime}, \beta^{\prime}}$, à coefficients dans $\mathbb{Q}(t)$ :

$$
R_{\boldsymbol{\Lambda}}=\sum_{\left(\alpha^{\prime}, \beta^{\prime}\right) \in \mathcal{P}_{n} \times \mathcal{P}_{m}} r_{\boldsymbol{\Lambda}}\left(\alpha^{\prime}, \beta^{\prime}\right) s_{\alpha^{\prime}, \beta^{\prime}}
$$

On doit prouver que, pour tout couple $\left(\alpha^{\prime}, \beta^{\prime}\right) \in \mathcal{P}_{n} \times \mathcal{P}_{m}$ tel que $r_{\boldsymbol{\Lambda}}\left(\alpha^{\prime}, \beta^{\prime}\right) \neq 0$, on a

$$
\text { (1) } \quad \Lambda\left(\alpha^{\prime}, \beta^{\prime}\right) \leq \Lambda \text {. }
$$

On commence par calculer les coefficients $r_{\Lambda}\left(\alpha^{\prime}, \beta^{\prime}\right)$. On note $\left(v_{i}^{0}\right)_{i=1, \ldots, n}$ la base canonique de $\mathbb{Z}^{n}$ et $\left(v_{i}^{1}\right)_{i=1, \ldots, m}$ celle de $\mathbb{Z}^{m}$. On identifie naturellement les éléments de ces bases à des éléments de $\mathbb{Z}^{n} \times \mathbb{Z}^{m}$. Notons $J$ le sous-ensemble de $\mathbb{Z}^{n} \times \mathbb{Z}^{m}$ formé des éléments $v_{i}^{e}-v_{j}^{f}$, pour les couples $((i, e),(j, f)) \in I^{2}$ tels que $e \neq f$, $(i, e)<(j, f)$ et $(i, e) \leq \nu_{0}$. Notons $K$ le sous-ensemble de $\mathbb{Z}^{n} \times \mathbb{Z}^{m}$ formé des éléments $v_{i}^{e}-v_{j}^{f}$, pour les couples $((i, e),(j, f)) \in I^{2}$ tels que $e=f,(i, e)<(j, f)$ et $\nu_{0}<(i, e)$. On pose $\Delta_{n}=(n-1, n-2, \ldots, 0)$ et on définit de même $\Delta_{m}$. Le couple $\left(\alpha+\Delta_{n}, \beta+\Delta_{m}\right)$ est un élément de $\mathbb{Z}^{n} \times \mathbb{Z}^{m}$. Le groupe $\mathfrak{S}_{n}$ agit sur $\mathbb{Z}^{n}$ : pour $x=\left(x_{1}, \ldots, x_{n}\right) \in \mathbb{Z}^{n}$ et $\sigma \in \mathfrak{S}_{n}$, on pose $x^{\sigma}=\left(x_{\sigma 1}, \ldots, x_{\sigma n}\right)$. De même, $\mathfrak{S}_{m}$ agit sur $\mathbb{Z}^{m}$. Soit $\left(\alpha^{\prime}, \beta^{\prime}\right) \in \mathcal{P}_{n} \times \mathcal{P}_{m}$. Pour $d \in \mathbb{N}$, posons

$$
\begin{gathered}
(2) \quad r_{\Lambda}^{d}\left(\alpha^{\prime}, \beta^{\prime}\right)=\sum_{\sigma \in \mathfrak{S}_{n}, \tau \in \mathfrak{S}_{m}} \operatorname{sgn}(\sigma) \operatorname{sgn}(\tau) \\
\left|\left\{X \subset J \cup K ;|X|=d,\left(\alpha+\Delta_{n}, \beta+\Delta_{m}\right)-\sum_{x \in X} x=\left(\left(\alpha^{\prime}+\Delta_{n}\right)^{\sigma},\left(\beta^{\prime}+\Delta_{m}\right)^{\tau}\right)\right\}\right| .
\end{gathered}
$$

D'après 4] 3.13.1, on a l'égalité

$$
r_{\Lambda}\left(\alpha^{\prime}, \beta^{\prime}\right)=v_{\alpha, \beta}(t)^{-1} \sum_{d \in \mathbb{N}}(-1)^{d} r_{\Lambda}^{d}\left(\alpha^{\prime}, \beta^{\prime}\right) t^{d}
$$

où $v_{\alpha, \beta}(t)$ est un certain polynôme non nul. On peut récrire l'expression (2) sous la forme

$$
r_{\Lambda}^{d}\left(\alpha^{\prime}, \beta^{\prime}\right)=\sum_{\sigma \in \mathfrak{S}_{n}, \tau \in \mathfrak{S}_{m}} \operatorname{sgn}(\sigma) \operatorname{sgn}(\tau) \sum_{X_{J} \subset J ;\left|X_{J}\right| \leq d}\left|\mathcal{X}_{K}\left(X_{J}, \sigma, \tau\right)\right|,
$$

où

$$
\begin{gathered}
\mathcal{X}_{K}\left(X_{J}, \sigma, \tau\right)=\left\{X_{K} \subset K ;\left|X_{K}\right|=d-\left|X_{J}\right|\right. \\
\left.\left(\alpha+\Delta_{n}, \beta+\Delta_{m}\right)-\sum_{x \in X_{K}} x=\left(\left(\alpha^{\prime}+\Delta_{n}\right)^{\sigma},\left(\beta^{\prime}+\Delta_{m}\right)^{\tau}\right)+\sum_{x \in X_{J}} x\right\} .
\end{gathered}
$$

Introduisons le plus petit nombre $l \in\{0, \ldots, n\}$ tel que $(n-l, 0) \leq \nu_{0}$ et le plus petit nombre $h \in\{0, \ldots, m\}$ tel que $(m-h, 1) \leq \nu_{0}$. Pour $y=\left(y_{1}, \ldots, y_{n}\right) \in \mathbb{Z}^{n}$, on pose $y_{\leq n-l}=\left(y_{1}, \ldots, y_{n-l}\right)$ et $y_{>n-l}=\left(y_{n-l+1}, \ldots, y_{n}\right)$. Pour $y \in \mathbb{Z}^{m}$, on définit de même $y_{\leq m-h}$ et $y_{>m-h}$. Pour $y=\left(y^{0}, y^{1}\right) \in \mathbb{Z}^{n} \times \mathbb{Z}^{m}$, on pose $p_{\leq}(y)=\left(y_{\leq n-l}^{0}, y_{\leq m-h}^{1}\right)$ et 
$p_{>}(y)=\left(y_{>n-l}^{0}, y_{>m-h}^{1}\right)$. Remarquons que, pour $y \in K$, on a $p_{\leq}(y)=0$. Donc $K$ s'identifie par $p_{>}$à un sous-ensemble de $\mathbb{Z}^{l} \times \mathbb{Z}^{h}$. De plus, on a $p_{>}\left(\alpha+\Delta_{n}, \beta+\Delta_{m}\right)=$ $\left(\Delta_{l}, \Delta_{h}\right)$. Pour $J, \sigma$ et $\tau$ comme ci-dessus, l'ensemble $\mathcal{X}_{K}\left(X_{J}, \sigma, \tau\right)$ est donc vide si la condition

(5) $p_{\leq}\left(\alpha+\Delta_{n}, \beta+\Delta_{m}\right)=p_{\leq}\left(\left(\left(\alpha^{\prime}+\Delta_{n}\right)^{\sigma},\left(\beta^{\prime}+\Delta_{m}\right)^{\tau}\right)+\sum_{x \in X_{J}} x\right)$ n'est pas vérifiée. Si elle l'est, $\mathcal{X}_{K}\left(X_{J}, \sigma, \tau\right)$ s'identifie à l'ensemble des $X_{K} \subset K$ tels que $\left|X_{K}\right|=d-\left|X_{J}\right|$ et

$$
\left(\Delta_{l}, \Delta_{h}\right)-\sum_{x \in X_{K}} x=p_{>}\left(\left(\left(\alpha^{\prime}+\Delta_{n}\right)^{\sigma},\left(\beta^{\prime}+\Delta_{m}\right)^{\tau}\right)+\sum_{x \in X_{J}} x\right) .
$$

Notons $\mathfrak{S}\left[X_{J}\right]$ l'ensemble des $(\sigma, \tau) \in \mathfrak{S}_{n} \times \mathfrak{S}_{m}$ tels que (5) soit vérifiée. Supposons que $(\sigma, \tau)$ appartienne à cet ensemble. Par cohérence avec ce qui précède, un élément de $\mathbb{Z}^{l}$ sera noté $y=\left(y_{n-l+1}, \ldots, y_{n}\right)$. On utilise les mêmes notations qu'en 3.2. L'ensemble $\mathcal{X}_{K}\left(X_{J}, \sigma, \tau\right)$ se décompose en la réunion sur les $z \in\left(\mathbb{Z}^{l}\right)^{+}, \sigma^{\prime} \in \operatorname{stab}(z) \backslash \mathfrak{S}_{l}$, $y \in\left(\mathbb{Z}^{h}\right)^{+}, \tau^{\prime} \in \operatorname{stab}(y) \backslash \mathfrak{S}_{h}$ du sous-ensemble des $X_{K} \in \mathcal{X}_{K}\left(X_{J}, \sigma, \tau\right)$ tels que

$$
\left(\Delta_{h}, \Delta_{l}\right)-\sum_{x \in X_{K}} x=\left(z^{\sigma^{\prime}}, y^{\tau^{\prime}}\right)
$$

Ce sous-ensemble est non vide seulement si

$$
\left(z^{\sigma^{\prime}}, y^{\tau^{\prime}}\right)=p_{>}\left(\left(\left(\alpha^{\prime}+\Delta_{n}\right)^{\sigma},\left(\beta^{\prime}+\Delta_{m}\right)^{\tau}\right)+\sum_{x \in X_{J}} x\right)
$$

Cette condition ne dépend pas de l'ensemble $X_{K}$. Pour $\sigma^{\prime} \in \mathfrak{S}_{l}$ et $\tau^{\prime} \in \mathfrak{S}_{h}$, notons $\mathcal{Z}\left(\sigma^{\prime}, \tau^{\prime}, \sigma, \tau, X_{J}\right)$ l'ensemble des $(z, y) \in\left(\mathbb{Z}^{l}\right)^{+} \times\left(\mathbb{Z}^{h}\right)^{+}$qui vérifient $(7)$. D'autre part, pour $(z, y) \in\left(\mathbb{Z}^{l}\right)^{+} \times\left(\mathbb{Z}^{h}\right)^{+}, \sigma^{\prime} \in \mathfrak{S}_{l}, \tau^{\prime} \in \mathfrak{S}_{h}$ et $f \in \mathbb{N}$, notons $\mathcal{X}_{K}\left(f, z, y, \sigma^{\prime}, \tau^{\prime}\right)$ l'ensemble des $X_{K} \subset K$ qui vérifient (6) et ont $f$ éléments. Si $(z, y) \notin \mathcal{Z}\left(\sigma^{\prime}, \tau^{\prime}, \sigma, \tau, X_{J}\right)$, l'ensemble des $X_{K} \in \mathcal{X}_{K}\left(X_{J}, \sigma, \tau\right)$ qui vérifient (6) est vide. Si $(z, y) \in \mathcal{Z}\left(\sigma^{\prime}, \tau^{\prime}, \sigma, \tau, X_{J}\right)$, cet ensemble est égal à $\mathcal{X}_{K}\left(d-\left|X_{J}\right|, z, y, \sigma^{\prime}, \tau^{\prime}\right)$. En rassemblant ces calculs, on obtient la formule suivante

$$
\text { (8) } \quad r_{\Lambda}^{d}\left(\alpha^{\prime}, \beta^{\prime}\right)=
$$

$$
\begin{gathered}
\sum_{X_{J} \subset J,\left|X_{J}\right| \leq d} \sum_{(\sigma, \tau) \in \mathfrak{S}\left[X_{J}\right]} \operatorname{sgn}(\sigma) \operatorname{sgn}(\tau) \sum_{\sigma^{\prime} \in \mathfrak{S}_{l}, \tau^{\prime} \in \mathfrak{S}_{h}} \sum_{(z, y) \in \mathcal{Z}\left(\sigma^{\prime}, \tau^{\prime}, \sigma, \tau, X_{J}\right)} \\
|\operatorname{stab}(z)|^{-1}|\operatorname{stab}(y)|^{-1}\left|\mathcal{X}_{K}\left(d-\left|X_{J}\right|, z, y, \sigma^{\prime}, \tau^{\prime}\right)\right| .
\end{gathered}
$$

On plonge naturellement $\mathfrak{S}_{l}$ dans $\mathfrak{S}_{n}$ : le groupe $\mathfrak{S}_{l}$ agit sur les $l$ dernières coordonnées. De même, on plonge $\mathfrak{S}_{h}$ dans $\mathfrak{S}_{m}$. On s'aperçoit que l'action de $\mathfrak{S}_{l} \times \mathfrak{S}_{h}$ conserve l'ensemble $J$. On en déduit une action $X_{J} \mapsto X_{J}^{\sigma^{\prime}, \tau^{\prime}}$ sur l'ensemble des sous-ensembles de $X_{J}$. La relation (7) équivaut à

$$
(z, y)=p_{>}\left(\left(\left(\alpha^{\prime}+\Delta_{n}\right)^{\sigma \sigma^{\prime-1}},\left(\beta^{\prime}+\Delta_{m}\right)^{\tau \tau^{\prime-1}}\right)+\sum_{x \in X_{J}^{\sigma^{\prime-1}, \tau^{\prime-1}}} x\right)
$$

Autrement dit $\mathcal{Z}\left(\sigma^{\prime}, \tau^{\prime}, \sigma, \tau, X_{J}\right)=\mathcal{Z}\left(1,1, \sigma{\sigma^{\prime}}^{-1}, \tau{\tau^{\prime}}^{-1}, X_{J}^{\sigma^{\prime-1}}, \tau^{\prime-1}\right)$. D'autre part, l'action de $\mathfrak{S}_{l} \times \mathfrak{S}_{h}$ ne change pas l'image d'un élément de $\mathbb{Z}^{n} \times \mathbb{Z}^{m}$ par la projection $p_{\leq}$. Donc $(\sigma, \tau) \in \mathfrak{S}\left[X_{J}\right]$ si et seulement si $\left(\sigma \sigma^{\prime-1}, \tau \tau^{\prime-1}\right) \in \mathfrak{S}\left[X_{J}^{\sigma^{\prime-1}, \tau^{\prime-1}}\right]$. Dans la formule (8), on intervertit les sommes en commençant par sommer en $\sigma^{\prime}, \tau^{\prime}$. On remplace ensuite $X_{J}$ par $X_{J}^{\sigma^{\prime}, \tau^{\prime}}$ et $(\sigma, \tau) \operatorname{par}\left(\sigma \sigma^{\prime}, \tau \tau^{\prime}\right)$. Ceci fait apparaître 
des termes $\operatorname{sgn}\left(\sigma^{\prime}\right) \operatorname{sgn}\left(\tau^{\prime}\right)$. On utilise les propriétés ci-dessus et on intervertit de nouveau les sommes. On obtient alors

(9) $\quad r_{\Lambda}^{d}\left(\alpha^{\prime}, \beta^{\prime}\right)=$

$$
\begin{gathered}
\sum_{X_{J} \subset J,\left|X_{J}\right| \leq d} \sum_{(\sigma, \tau) \in \mathfrak{S}\left[X_{J}\right]} \operatorname{sgn}(\sigma) \operatorname{sgn}(\tau) \sum_{(z, y) \in \mathcal{Z}\left(1,1, \sigma, \tau, X_{J}\right)}|\operatorname{stab}(z)|^{-1}|\operatorname{stab}(y)|^{-1} \\
\sum_{\sigma^{\prime} \in \mathfrak{S}_{l}, \tau^{\prime} \in \mathfrak{S}_{h}} \operatorname{sgn}\left(\sigma^{\prime}\right) \operatorname{sgn}\left(\tau^{\prime}\right)\left|\mathcal{X}_{K}\left(d-\left|X_{J}\right|, z, y, \sigma^{\prime}, \tau^{\prime}\right)\right| .
\end{gathered}
$$

Posons

$$
v_{l}(t)=\prod_{i=1, \ldots, l} \frac{1-t^{i}}{1-t}
$$

et définissons de même $v_{h}(t)$. Notons $c_{f}$ le coefficient de $t^{f}$ dans $v_{h}(t) v_{l}(t)$. Montrons que, pour $f \in \mathbb{N}$ et $(z, y) \in\left(\mathbb{Z}^{l}\right)^{+} \times\left(\mathbb{Z}^{h}\right)^{+}$, on a

$$
\begin{aligned}
& \sum_{\sigma^{\prime} \in \mathfrak{S}_{l}, \tau^{\prime} \in \mathfrak{S}_{h}} \operatorname{sgn}\left(\sigma^{\prime}\right) \operatorname{sgn}\left(\tau^{\prime}\right)\left|\mathcal{X}_{K}\left(f, z, y, \sigma^{\prime}, \tau^{\prime}\right)\right| \\
= & \left\{\begin{array}{cl}
0, & \text { si }(z, y) \neq\left(\Delta_{l}, \Delta_{h}\right), \\
(-1)^{f} c_{f}, & \text { si }(z, y)=\left(\Delta_{l}, \Delta_{h}\right) .
\end{array}\right.
\end{aligned}
$$

En reprenant les définitions, on voit que la somme à calculer est égale au produit de $(-1)^{f}$ et du coefficient de $X_{n-l+1}^{z_{n-1+1}} \ldots X_{n}^{z_{n}} Y_{m-h+1}^{y_{m-h+1}} \ldots Y_{m}^{y_{m}} t^{f}$ dans le polynôme $P\left(X_{n-l+1}, \ldots, X_{n}, t\right) Q\left(Y_{m-h+1}, \ldots, Y_{m}, t\right)$, où

$$
\begin{aligned}
& P\left(X_{n-l+1}, \ldots, X_{n}, t\right)=\sum_{\sigma^{\prime} \in \mathfrak{S}_{l}} \operatorname{sgn}\left(\sigma^{\prime}\right)\left(\prod_{i=n-l+1, \ldots, n} X_{\sigma^{\prime}(n-l+1)}^{l-1} \ldots X_{\sigma^{\prime}(n-1)}\right) \\
& \prod_{n-l+1 \leq i<j \leq n}\left(1-t X_{\sigma^{\prime}(j)} X_{\sigma^{\prime}(i)}^{-1}\right), \\
& Q\left(Y_{m-h+1}, \ldots, Y_{m}, t\right)=\sum_{\tau^{\prime} \in \mathfrak{S}_{h}} \operatorname{sgn}\left(\tau^{\prime}\right)\left(\prod_{i=m-h+1, \ldots, m} Y_{\tau^{\prime}(m-h+1)}^{h-1} \ldots Y_{\tau^{\prime}(m-1)}\right) \\
& \prod_{m-h+1 \leq i<j \leq m}\left(1-t Y_{\tau^{\prime}(j)} Y_{\tau^{\prime}(i)}^{-1}\right) .
\end{aligned}
$$

On récrit

$$
P\left(X_{n-l+1}, \ldots, X_{n}, t\right)=\sum_{\sigma^{\prime} \in \mathfrak{S}_{l}} \operatorname{sgn}\left(\sigma^{\prime}\right) \prod_{n-l+1 \leq i<j \leq n}\left(X_{\sigma^{\prime}(i)}-t X_{\sigma^{\prime}(j)}\right) .
$$

On sait que

$$
\prod_{n-l+1 \leq i<j \leq n}\left(X_{\sigma^{\prime}(i)}-X_{\sigma^{\prime}(j)}\right)=\operatorname{sgn}\left(\sigma^{\prime}\right) \prod_{n-l+1 \leq i<j \leq n}\left(X_{i}-X_{j}\right) .
$$

On a donc aussi

$$
P\left(X_{n-l+1}, \ldots, X_{n}, t\right)=\left(\prod_{n-l+1 \leq i<j \leq n}\left(X_{i}-X_{j}\right)\right) \sum_{\sigma^{\prime} \in \mathfrak{S}_{l}} \prod_{n-l+1 \leq i<j \leq n} \frac{X_{\sigma^{\prime}(i)}-t X_{\sigma^{\prime}(j)}}{X_{\sigma^{\prime}(i)}-X_{\sigma^{\prime}(j)}}
$$

D'après [3] III.(1.4), on a

$$
\sum_{\sigma^{\prime} \in \mathfrak{S}_{l}} \prod_{n-l+1 \leq i<j \leq n} \frac{X_{\sigma^{\prime}(i)}-t X_{\sigma^{\prime}(j)}}{X_{\sigma^{\prime}(i)}-X_{\sigma^{\prime}(j)}}=v_{l}(t)
$$


d'où

$$
P\left(X_{n-l+1}, \ldots, X_{n}, t\right)=v_{l}(t) \prod_{n-l+1 \leq i<j \leq n}\left(X_{i}-X_{j}\right) .
$$

Puisque $z$ appartient à $\left(\mathbb{Z}^{l}\right)^{+}$, il est bien connu que le coefficient de $X_{n-l+1}^{z_{n-l+1}} \ldots X_{n}^{z_{n}}$ dans ce polynôme est nul sauf si $z=\Delta_{l}$, auquel cas ce coefficient vaut $v_{l}(t)$. Un même calcul s'applique à $Q\left(Y_{m-h+1}, \ldots, Y_{m}, t\right)$. La relation (10) s'ensuit.

On utilise (10) pour simplifier (9). Seul le couple $(z, y)=\left(\Delta_{l}, \Delta_{h}\right)$ peut contribuer. Pour ce couple, $|\operatorname{stab}(z)|=|\operatorname{stab}(y)|=1$. Ce couple contribue si et seulement s'il appartient à $\mathcal{Z}\left(1,1, \sigma, \tau, X_{J}\right)$, autrement dit si

$$
\left(\Delta_{l}, \Delta_{h}\right)=p_{>}\left(\left(\left(\alpha^{\prime}+\Delta_{n}\right)^{\sigma},\left(\beta^{\prime}+\Delta_{m}\right)^{\tau}\right)+\sum_{x \in X_{J}} x\right) .
$$

Mais la conjonction de cette relation et de la condition que $(\sigma, \tau)$ appartienne à $\mathfrak{S}\left[X_{J}\right]$, c'est-à-dire que la relation (5) soit vérifiée, équivaut à l'égalité

$$
\left(\alpha+\Delta_{n}, \beta+\Delta_{m}\right)=\left(\left(\alpha^{\prime}+\Delta_{n}\right)^{\sigma},\left(\beta^{\prime}+\Delta_{m}\right)^{\tau}\right)+\sum_{x \in X_{J}} x .
$$

Pour tous $\sigma \in \mathfrak{S}_{n}, \tau \in \mathfrak{S}_{m}$ et $f \in \mathbb{N}$, notons $\mathcal{X}_{J}(f, \sigma, \tau)$ l'ensemble des sousensembles $X_{J} \subset J$ tels que $\left|X_{J}\right|=f$ et que (11) soit vérifiée. On obtient

$$
r_{\Lambda}^{d}\left(\alpha^{\prime}, \beta^{\prime}\right)=\sum_{\sigma \in \mathfrak{S}_{n}, \tau \in \mathfrak{S}_{m}} \operatorname{sgn}(\sigma) \operatorname{sgn}(\tau) \sum_{f=0, \ldots, d}(-1)^{d-f} c_{d-f}\left|\mathcal{X}_{J}(f, \sigma, \tau)\right| .
$$

En reportant cette expression dans (3), on obtient

$$
r_{\boldsymbol{\Lambda}}\left(\alpha^{\prime}, \beta^{\prime}\right)=v_{\alpha, \beta}(t)^{-1} v_{l}(t) v_{h}(t) \sum_{f \in \mathbb{N}}(-1)^{f} s_{\Lambda}^{f}\left(\alpha^{\prime}, \beta^{\prime}\right) t^{f},
$$

où

$$
s_{\boldsymbol{\Lambda}}^{f}\left(\alpha^{\prime}, \beta^{\prime}\right)=\sum_{\sigma \in \mathfrak{S}_{n}, \tau \in \mathfrak{S}_{m}} \operatorname{sgn}(\sigma) \operatorname{sgn}(\tau)\left|\mathcal{X}_{J}(f, \sigma, \tau)\right| .
$$

La formule (12) est similaire à (3), on s'est simplement débarrassé de l'ensemble $K$.

4.4. Fin de la démonstration de la proposition 4.2, A ce point, nous allons utiliser l'identification de $I$ à $\{1, \ldots, 2 r+1\}$ définie en 2.11: on identifie $(j, 0)$ à $x_{j}$ et $(l, 1)$ à $y_{l}$. Cette identification induit un isomorphisme $\iota: \mathbb{R}^{n} \times \mathbb{R}^{m} \simeq \mathbb{R}^{2 r+1}$. Les couples $\left(\alpha^{\prime}, \beta^{\prime}\right) \in \mathcal{P}_{n} \times \mathcal{P}_{m}$ s'identifient ainsi à des éléments de $\mathbb{R}^{2 r+1}$. On note $I_{a}=\left\{x_{1}, \ldots, x_{n}\right\}$ et $I_{b}=\left\{y_{1}, \ldots, y_{m}\right\}$. Introduisons le groupe $G=G L(2 r+1, \mathbb{C})$, son sous-tore diagonal $T$ et le groupe $X^{*}(T)$ des caractères algébriques de $T$. On a naturellement $X^{*}(T) \otimes_{\mathbb{Z}} \mathbb{R}=\mathbb{R}^{2 r+1}$. On note $V=\mathbb{R}^{2 r+1}$ et $\left(\epsilon_{i}\right)_{i=1, \ldots, 2 r+1}$ la base standard de cet espace. La décomposition $\{1, \ldots, 2 r+1\}=I_{a} \sqcup I_{b}$ définit un sous-groupe de Levi semi-standard $M$ de $G$ : les éléments de $M$ conservent les deux sous-espaces de $\mathbb{C}^{2 r+1}$ engendrés par les $\epsilon_{i}$ pour $i \in I_{a}$, resp. $i \in I_{b}$. On introduit la chambre positive fermée $\bar{C}^{+}$associée au sous-groupe de Borel triangulaire supérieur de $G$. Autrement dit $\bar{C}^{+}$est l'ensemble des $x=\left(x_{1}, \ldots, x_{2 r+1}\right) \in V$ tels que $x_{1} \geq$ $\ldots \geq x_{2 r+1}$. Notons $W=\mathfrak{S}_{2 r+1}$ le groupe de Weyl de $G$ relatif à $T$ et $W^{M}$ le groupe de Weyl de $M$. Via le plongement $\iota$, le groupe $\mathfrak{S}_{n} \times \mathfrak{S}_{m}$ du paragraphe précédent s'identifie à $W^{M}$. Comme on le sait, pour tout $x \in V$, il existe un unique élément de $\bar{C}^{+}$, que l'on note $x^{+}$, de sorte qu'il existe $w \in W$ tel que $w(x)=x^{+}$. On introduit l'ensemble $\Sigma$ des racines $\epsilon_{i}-\epsilon_{j}$ pour $i \neq j$ et le sous-ensemble $\Sigma^{+}$des racines positives, c'est-à-dire des $\epsilon_{i}-\epsilon_{j}$ pour $i<j$. On note aussi $\Sigma^{M}$ l'ensemble des racines dans $M$, c'est-à-dire les $\epsilon_{i}-\epsilon_{j}$ pour $i \neq j, i, j \in I_{a}$ ou $i, j \in I_{b}$. On pose $\Sigma^{M,+}=$ 
$\Sigma^{M} \cap \Sigma^{+}$. Pour tout entier $e \in \mathbb{N}$, posons $\delta_{e}=\{(e-1) / 2,(e-3) / 2, \ldots,(1-e) / 2\}$. L'élément $\delta_{2 r+1}$ est égal à la demi-somme des racines positives et $\iota\left(\delta_{n}, \delta_{m}\right)$ est égal à la demi-somme des racines dans $\Sigma^{M,+}$. Nous noterons ces éléments $\delta$ et $\delta^{M}$. Posons $\mathcal{J}=\iota(J)$. On voit que $\mathcal{J}$ est un sous-ensemble de $\Sigma^{+}-\Sigma^{M,+}$. On note $z$ l'élément de $\mathbb{R}^{2 r+1}$ défini par

si $k$ est pair, $z_{i}=n-1$ pour $i \in I_{a}$ et $z_{i}=m$ pour $i \in I_{b}$;

si $k$ est impair, $z_{i}=n$ pour $i \in I_{a}$ et $z_{i}=m-1$ pour $i \in I_{b}$.

Avec ces définitions, on voit que, pour $\left(\alpha^{\prime}, \beta^{\prime}\right) \in \mathcal{P}_{n} \times \mathcal{P}_{m}, \iota\left(\Lambda\left(\alpha^{\prime}, \beta^{\prime}\right)\right)=$ $\iota\left(\alpha^{\prime}, \beta^{\prime}\right)+2 \delta^{M}+z$ et $\Lambda\left(\alpha^{\prime}, \beta^{\prime}\right)=\iota\left(\boldsymbol{\Lambda}\left(\alpha^{\prime}, \beta^{\prime}\right)\right)^{+}$. Remarquons que l'ordre $<$a été défini de sorte que $\iota(\boldsymbol{\Lambda})=\iota(\boldsymbol{\Lambda}(\alpha, \beta))$ appartienne à $\bar{C}^{+}$, autrement dit $\Lambda=\Lambda(\alpha, \beta)=$ $\iota(\boldsymbol{\Lambda}(\alpha, \beta))$. A ce point, on se rappelle l'hypothèse que $\Lambda$ est sans multiplicités. Puisque les termes de $\Lambda$ sont des entiers, on a $\Lambda_{i} \geq \Lambda_{i+1}+1$ pour tout $i=1, \ldots, 2 r$, ce qui entraîne

(1) $\Lambda-\rho \in \bar{C}^{+}$.

Soit $\left(\alpha^{\prime}, \beta^{\prime}\right) \in \mathcal{P}_{n} \times \mathcal{P}_{m}$, supposons $r_{\boldsymbol{\Lambda}}\left(\alpha^{\prime}, \beta^{\prime}\right) \neq 0$. D'après 4.3 (12), on peut fixer $\sigma \in \mathfrak{S}_{n}, \tau \in \mathfrak{S}_{m}$ et $X_{J} \subset J$ de sorte que 4.3.(11) soit vérifiée. On voit que, dans cette relation, on peut aussi bien remplacer $\Delta_{n}$ et $\Delta_{m}$ par $\delta_{n}$ et $\delta_{m} \operatorname{car}\left(\Delta_{n}-\delta_{n}, \Delta_{m}-\delta_{m}\right)$ est fixe par $\sigma \times \tau$. En posant $X=\iota\left(X_{J}\right), v=\iota(\alpha, \beta), v^{\prime}=\iota\left(\alpha^{\prime}, \beta^{\prime}\right)$ et en notant $w^{M}$ l'élément de $W^{M}$ auquel s'identifie $\sigma \times \tau$, cette relation s'écrit

$$
v^{\prime}+\delta^{M}=w^{M}\left(v+\delta^{M}-\sum_{x \in X} x\right) .
$$

Alors

$$
\begin{aligned}
\iota\left(\boldsymbol{\Lambda}\left(\alpha^{\prime}, \beta^{\prime}\right)\right) & =v^{\prime}+2 \delta^{M}+z=w^{M}\left(v+\delta^{M}-\sum_{x \in X} x\right)+\delta^{M}+z \\
& =w^{M}\left(\Lambda-\delta^{M}-z-\sum_{x \in X} x\right)+\delta^{M}+z .
\end{aligned}
$$

L'élément $z$ disparaît de cette relation : il est central dans $M$ et fixe par $w^{M}$. Pour démontrer que $\Lambda\left(\alpha^{\prime}, \beta^{\prime}\right) \leq \Lambda$, cf. 4.3(1), on est ramené au problème suivant. L'élément $\Lambda$ vérifie (1). On se donne un sous-ensemble $X \subset \Sigma^{+}-\Sigma^{M,+}$ et un élément $w^{M} \in W^{M}$. On pose

$$
\underline{\Lambda}=w^{M}\left(\Lambda-\delta^{M}-\sum_{x \in X} x\right)+\delta^{M}
$$

et on veut prouver

$$
\text { (2) } \quad \underline{\Lambda}^{+} \leq \Lambda
$$

On a

$$
\delta-\delta^{M}=\frac{1}{2} \sum_{x \in \Sigma^{+}-\Sigma^{M,+}} x=\frac{1}{2}\left(\sum_{x \in X} x+\sum_{x \in \Sigma^{+}-\left(\Sigma^{M,+} \cup X\right)} x\right) .
$$

D'où $\delta^{M}+\sum_{x \in X} x=\delta+\mu$, où

$$
\mu=\frac{1}{2}\left(\sum_{x \in X} x-\sum_{x \in \Sigma^{+}-\left(\Sigma^{M},+\cup X\right)} x\right) .
$$

On a

$$
\underline{\Lambda}=w^{M}(\Lambda-\delta-\mu)+\delta^{M}=w^{M}\left(\Lambda-\delta+\mu^{\prime}\right),
$$

où $\mu^{\prime}=-\mu+\left(w^{M}\right)^{-1}\left(\delta^{M}\right)$. Par définition, l'élément $\underline{\Lambda}^{+}$est insensible à la transformation de $\underline{\Lambda}$ par n'importe quel élément de $W$. Cela entraîne

$$
\underline{\Lambda}^{+}=\left(\Lambda-\delta+\mu^{\prime}\right)^{+} \text {. }
$$


Notons ${ }^{+} \bar{C}$ le cône fermé engendré par les éléments de $\Sigma^{+}$. Pour prouver (2), il suffit de prouver que $\left(\Lambda-\delta+\mu^{\prime}\right)^{+} \in \Lambda-{ }^{+} \bar{C}$. Par définition, il existe $w \in W$ tel que $\left(\Lambda-\delta+\mu^{\prime}\right)^{+}=w\left(\Lambda-\delta+\mu^{\prime}\right)$. D'où

$\left(\Lambda-\delta+\mu^{\prime}\right)^{+}=w(\Lambda-\delta)+(\Lambda-\delta)-(\Lambda-\delta)+w\left(\mu^{\prime}\right)=\Lambda+w(\Lambda-\delta)-(\Lambda-\delta)+w\left(\mu^{\prime}\right)-\delta$.

Puisque $\Lambda-\delta \in \bar{C}^{+}$, il est bien connu que $w(\Lambda-\delta)-(\Lambda-\delta)$ appartient à $-{ }^{+} \bar{C}$. Il reste à prouver que $w\left(\mu^{\prime}\right)-\delta$ appartient aussi à ce cône. Notons $E$ l'ensemble des éléments de $V$ qui s'écrivent $\frac{1}{2}\left(\sum_{y \in Y} y-\sum_{y \in \Sigma^{+}-Y} y\right)$ pour un sous-ensemble $Y \subset \Sigma^{+}$et notons $E^{M}$ l'ensemble des éléments de $V$ qui s'écrivent $\frac{1}{2}\left(\sum_{y \in Y} y-\sum_{y \in \Sigma^{M,+}{ }_{-Y}} y\right)$ pour un sous-ensemble $Y \subset \Sigma^{M,+}$. En utilisant (3), on voit que $-\mu+H \in E$ pour tout $H \in E^{M}$. D'autre part, $E$ est conservé par l'action de $W$ et $E^{M}$ l'est par celle de $W^{M}$. Puisque $\delta^{M}$ appartient à $E^{M}$, l'élément $\mu^{\prime}=-\mu+\left(w^{M}\right)^{-1}\left(\delta^{M}\right)$ appartient à $E$ et aussi $w\left(\mu^{\prime}\right) \in E$. Mais on voit que $H-\delta$ appartient à $-{ }^{+} \bar{C}$ pour tout $H \in E$. En particulier $w\left(\mu^{\prime}\right)-\delta$ appartient à $-{ }^{+} \bar{C}$, ce qui achève la démonstration.

\subsection{Un théorème de maximalité.}

Théorème. Soit $(\lambda, \epsilon) \in \mathcal{P}^{\text {symp }}(2 N)$. Supposons que $\lambda$ n'a que des termes pairs. Alors il existe un unique élément $\left(\lambda^{\max }, \epsilon^{\max }\right) \in \mathcal{P}^{\text {symp }}(2 N)$ vérifiant les propriétés suivantes :

(i) $\mathfrak{m u l t}\left(\lambda, \epsilon ; \lambda^{\max }, \epsilon^{\max }\right)=1$;

(ii) pour tout élément $\left(\lambda^{\prime}, \epsilon^{\prime}\right) \in \mathcal{P}^{\text {symp }}(2 N)$ tel que $\mathfrak{m u l t}\left(\lambda, \epsilon ; \lambda^{\prime}, \epsilon^{\prime}\right) \neq 0$, on a $\lambda^{\prime}<\lambda^{\max }$ ou $\left(\lambda^{\prime}, \epsilon^{\prime}\right)=\left(\lambda^{\max }, \epsilon^{\max }\right)$.

Preuve. Posons $k=k(\lambda, \epsilon)$. Puisqu'on se préoccupe de paires $\left(\lambda^{\prime}, \epsilon^{\prime}\right)$ telles que $\mathfrak{m u l t}\left(\lambda, \epsilon ; \lambda^{\prime}, \epsilon^{\prime}\right) \neq 0$, on peut se limiter au sous-ensemble $\mathcal{P}^{\text {symp }}(2 N)_{k}$ des éléments $\left(\lambda^{\prime}, \epsilon^{\prime}\right)$ tels que $k\left(\lambda^{\prime}, \epsilon^{\prime}\right)=k$. Montrons tout d'abord que, pour $\left(\lambda^{\prime}, \epsilon^{\prime}\right),\left(\lambda^{\prime \prime}, \epsilon^{\prime \prime}\right) \in$ $\mathcal{P}^{\operatorname{symp}}(2 N)_{k}$, on a l'équivalence

(1) $\lambda^{\prime} \leq \lambda^{\prime \prime}$ si et seulement si $p_{\lambda^{\prime}, \epsilon^{\prime}} \leq p_{\lambda^{\prime \prime}, \epsilon^{\prime \prime}}$.

On applique à $\lambda^{\prime}$ la construction de $p_{\lambda^{\prime}, \epsilon^{\prime}}$ rappelée en 4.1. Pour $c \in \mathbb{N}_{>0}$, on a

$$
S_{c}\left(\lambda^{\prime}\right)=S_{c}\left(\lambda^{\prime}+\left[-1,-\infty[1)-S_{c}([-1,-\infty[1) .\right.\right.
$$

On a décomposé $\lambda^{\prime}+\left[-1,-\infty\left[1\right.\right.$ en la réunion des suites $\left(2 z_{1}, 2 z_{2}, \ldots\right)$ et $\left(2 z_{1}^{\prime}+\right.$ $\left.1,2 z_{2}^{\prime}+1, \ldots\right)$. On vérifie par récurrence les propriétés suivantes :

pour $c$ pair, les $c$ plus grands termes de $\lambda^{\prime}+\left[-1,-\infty\left[1\right.\right.$ sont les $2 z_{i}$ et $2 z_{i}^{\prime}+1$ pour $i=1, \ldots, c / 2$, à l'exception du cas où $S_{c}\left(\lambda^{\prime}\right)$ est impair ; dans ce cas, $\lambda_{c}^{\prime}$ est forcément impair, les $c$ plus grands termes de $\lambda^{\prime}+\left[-1, \infty\left[1\right.\right.$ sont les $2 z_{i}$ pour $i=1, \ldots, c / 2-1$ et les $2 z_{i}^{\prime}+1$ pour $i=1, \ldots, c / 2+1$ et on a $z_{c / 2+1}^{\prime}=z_{c / 2}$;

pour $c$ impair, les $c$ plus grands termes de $\lambda^{\prime}+\left[-1,-\infty\left[1\right.\right.$ sont les $2 z_{i}$ pour $i=1, \ldots,(c-1) / 2$ et les $2 z_{i}^{\prime}+1$ pour $i=1, \ldots,(c+1) / 2$, à l'exception du cas où $S_{c}\left(\lambda^{\prime}\right)$ est impair ; dans ce cas $\lambda_{c}^{\prime}$ est forcément impair, les $c$ plus grands termes de $\lambda^{\prime}+\left[-1,-\infty\left[1\right.\right.$ sont les $2 z_{i}$ pour $i=1, \ldots,(c+1) / 2$ et les $2 z_{i}^{\prime}+1$ pour $i=1, \ldots,(c-1) / 2$ et on a $z_{(c+1) / 2}=z_{(c+1) / 2}^{\prime}+1$.

Posons $c^{+}=c^{-}=c / 2$ si $c$ est pair, $c^{+}=(c+1) / 2, c^{-}=(c-1) / 2$ si $c$ est impair. Posons aussi $\delta_{c}\left(\lambda^{\prime}\right)=1$ si $S_{c}\left(\lambda^{\prime}\right)$ est impair. Les propriétés ci-dessus entraînent l'égalité

$$
S_{c}\left(\lambda^{\prime}+\left[-1,-\infty[1)=2 S_{c^{+}}\left(z^{\prime}\right)+c^{+}+2 S_{c^{-}}(z)+\delta_{c}\left(\lambda^{\prime}\right) .\right.\right.
$$

On a

$$
S_{c^{+}}\left(z^{\prime}\right)=S_{c^{+}}\left(A_{\lambda^{\prime}}^{\sharp}\right)-S_{c^{+}}([1,-\infty[1),
$$




$$
S_{c^{-}}(z)=S_{c^{-}}\left(B_{\lambda^{\prime}}^{\sharp}\right)-S_{c^{-}}\left(\left[0,-\infty\left[{ }_{1}\right) .\right.\right.
$$

D'où

$$
S_{c}\left(\lambda^{\prime}\right)=2 S_{c^{+}}\left(A_{\lambda^{\prime}}^{\sharp}\right)+2 S_{c^{-}}\left(B_{\lambda^{\prime}}^{\sharp}\right)+\delta_{c}\left(\lambda^{\prime}\right)+C_{c},
$$

où $C_{c}$ est un nombre indépendant de $\lambda^{\prime}$. On vérifie qu'en notant $a_{j}^{\sharp}$ et $b_{j}^{\sharp}$ les termes de $A_{\lambda^{\prime}}^{\sharp}$ et $B_{\lambda^{\prime}}^{\sharp}$, on a

$$
a_{1}^{\sharp} \geq b_{1}^{\sharp} \geq a_{2}^{\sharp} \geq b_{2}^{\sharp} \geq \ldots
$$

Donc

$$
S_{c^{+}}\left(A_{\lambda^{\prime}}^{\sharp}\right)+S_{c^{-}}\left(B_{\lambda^{\prime}}^{\sharp}\right)=S_{c}\left(A_{\lambda^{\prime}}^{\sharp} \sqcup B_{\lambda^{\prime}}^{\sharp}\right)=S_{c}\left(p_{\lambda^{\prime}, \epsilon^{\prime}}\right) .
$$

D'où

(2) $\quad S_{c}\left(\lambda^{\prime}\right)=2 S_{c}\left(p_{\lambda^{\prime}, \epsilon^{\prime}}\right)+\delta_{c}\left(\lambda^{\prime}\right)+C_{c}$.

Supposons $\lambda^{\prime} \leq \lambda^{\prime \prime}$. Alors $S_{c}\left(\lambda^{\prime}\right) \leq S_{c}\left(\lambda^{\prime \prime}\right)$, d'où

$$
S_{c}\left(p_{\lambda^{\prime}, \epsilon^{\prime}}\right) \leq S_{c}\left(p_{\lambda^{\prime \prime}, \epsilon^{\prime \prime}}\right)+\delta_{c}\left(\lambda^{\prime \prime}\right) / 2-\delta_{c}\left(\lambda^{\prime}\right) / 2 \leq S_{c}\left(p_{\lambda^{\prime \prime}, \epsilon^{\prime \prime}}\right)+1 / 2 .
$$

Puisque les sommes intervenant ici sont entières, on en déduit

$$
S_{c}\left(p_{\lambda^{\prime}, \epsilon^{\prime}}\right) \leq S_{c}\left(p_{\lambda^{\prime \prime}, \epsilon^{\prime \prime}}\right) .
$$

Cette inégalité étant vérifiée pour tout $c \geq 1$, on conclut que $p_{\lambda^{\prime}, \epsilon^{\prime}} \leq p_{\lambda^{\prime \prime}, \epsilon^{\prime \prime}}$. Inversement supposons vérifiée cette dernière inégalité. Alors

$$
S_{c}\left(p_{\lambda^{\prime}, \epsilon^{\prime}}\right) \leq S_{c}\left(p_{\lambda^{\prime \prime}, \epsilon^{\prime \prime}}\right) .
$$

Avec (2), on en déduit

$$
S_{c}\left(\lambda^{\prime}\right) \leq S_{c}\left(\lambda^{\prime \prime}\right)+\delta_{c}\left(\lambda^{\prime}\right)-\delta_{c}\left(\lambda^{\prime \prime}\right) \leq S_{c}\left(\lambda^{\prime \prime}\right)+\delta_{c}\left(\lambda^{\prime}\right) .
$$

Si $S_{c}\left(\lambda^{\prime}\right)$ est pair, $\delta_{c}\left(\lambda^{\prime}\right)=0$, d'où l'inégalité

$$
S_{c}\left(\lambda^{\prime}\right) \leq S_{c}\left(\lambda^{\prime \prime}\right) .
$$

Supposons que $S_{c}\left(\lambda^{\prime}\right)$ soit impair et que (4) ne soit pas vérifiée. Alors (3) force l'égalité $S_{c}\left(\lambda^{\prime}\right)=S_{c}\left(\lambda^{\prime \prime}\right)+1$. Puisque $\lambda^{\prime}$ est symplectique, le fait que $S_{c}\left(\lambda^{\prime}\right)$ soit impair entraîne que $\lambda_{c}^{\prime}$ est impair, que $\lambda_{c}^{\prime}=\lambda_{c+1}^{\prime}$ et que $S_{c-1}\left(\lambda^{\prime}\right)$ et $S_{c+1}\left(\lambda^{\prime}\right)$ sont pairs. On peut appliquer (4) pour $c-1$ (dans le cas particulier $c=1$, (4) est triviale pour $c-1=0)$. Puisque $S_{c}\left(\lambda^{\prime}\right)=S_{c-1}\left(\lambda^{\prime}\right)+\lambda_{c}^{\prime}$ et $S_{c}\left(\lambda^{\prime \prime}\right)=S_{c-1}\left(\lambda^{\prime \prime}\right)+\lambda_{c}^{\prime \prime}$, cette inégalité (4) pour $c-1$ et l'égalité $S_{c}\left(\lambda^{\prime}\right)=S_{c}\left(\lambda^{\prime \prime}\right)+1$ entraînent $\lambda_{c}^{\prime} \geq \lambda_{c}^{\prime \prime}+1$. Puisque $\lambda_{c+1}^{\prime}=\lambda_{c}^{\prime}$ et $\lambda_{c+1}^{\prime \prime} \leq \lambda_{c}^{\prime \prime}$, on a aussi $\lambda_{c+1}^{\prime} \geq \lambda_{c+1}^{\prime \prime}+1$. Alors l'égalité $S_{c}\left(\lambda^{\prime}\right)=S_{c}\left(\lambda^{\prime \prime}\right)+1$ entraîne

$$
S_{c+1}\left(\lambda^{\prime}\right)=S_{c}\left(\lambda^{\prime}\right)+\lambda_{c+1}^{\prime} \geq S_{c}\left(\lambda^{\prime \prime}\right)+\lambda_{c+1}^{\prime \prime}+2>S_{c+1}\left(\lambda^{\prime \prime}\right) .
$$

Puisque $S_{c+1}\left(\lambda^{\prime}\right)$ est pair, les deux termes extrêmes sont égaux d'après (4) pour $c+1$. Cette contradiction invalide notre hypothèse, donc (4) est forcément vérifiée. Cette inégalité étant maintenant prouvée pour tout $c$, on en déduit $\lambda^{\prime} \leq \lambda^{\prime \prime}$, ce qui prouve (1).

Notons $(\alpha, \beta) \in \mathcal{P}_{2}(N-k(k+1) / 2)$ le couple correspondant à $(\lambda, \epsilon)$. La proposition 4.2 et la relation (1) permettent de récrire l'énoncé sous la forme

il existe un unique couple $\left(\alpha^{\max }, \beta^{\max }\right) \in \mathcal{P} \times \mathcal{P}$ vérifiant les conditions

(i) $\operatorname{mult}\left(\alpha, \beta,<_{\alpha, \beta, k} ; \alpha^{\max }, \beta^{\max }\right)=1$;

(ii) pour tout couple $\left(\alpha^{\prime}, \beta^{\prime}\right) \in \mathcal{P} \times \mathcal{P}$ tel que $\operatorname{mult}\left(\alpha, \beta,<_{\alpha, \beta, k} ; \alpha^{\prime}, \beta^{\prime}\right) \neq 0$, on a $\Lambda_{k,-k-1 ; 2}\left(\alpha^{\prime}, \beta^{\prime}\right)<\Lambda_{k,-k-1 ; 2}\left(\alpha^{\max }, \beta^{\max }\right)$ ou $\left(\alpha^{\prime}, \beta^{\prime}\right)=\left(\alpha^{\max }, \beta^{\max }\right)$. 
La proposition 3.1 entraîne que ceci est vérifié si et seulement si l'ensemble $P_{k,-k-1 ; 2}\left(\alpha, \beta,<_{\alpha, \beta, k}\right)$ a un unique élément et, dans ce cas, $\left(\alpha^{\max }, \beta^{\max }\right)$ est cet unique élément. Or cette unicité est justement affirmée par le lemme 2.11. Cela achève la démonstration.

4.6. Tensorisation par le caractère signature. Pour toute partition $\mu \in \mathcal{P}$, on définit sa transposée usuelle ${ }^{t} \mu=\left({ }^{t} \mu_{1},{ }^{t} \mu_{2}, \ldots\right)$. Rappelons que

(1) pour tout $i \in \mathbb{N}_{>0},{ }^{t} \mu_{i}$ est le nombre d'indices $h \in \mathbb{N}_{>0}$ tels que $\mu_{h} \geq i$.

Posons $R_{\mu}=\mu+\left[0,-\infty\left[{ }_{1}, R_{t}{ }_{\mu}={ }^{t} \mu+\left[0,-\infty\left[{ }_{1}\right.\right.\right.\right.$. Montrons que

(2) pour tout $x \in \mathbb{Z}$, on a l'égalité

$$
\text { mult }_{R_{\mu}}(x)+\text { mult }_{R_{t}}(1-x)=1 .
$$

Preuve. Supposons mult $_{R_{\mu}}(x)=1$. Il existe alors un unique $j \in \mathbb{N}_{>0}$ tel que $x=\mu_{j}+1-j$. Cela entraîne à la fois $x+j \geq 1$ et $x+j>\mu_{j}$. Donc, d'après (1), ${ }^{t} \mu_{x+j}<j$, puis ${ }^{t} \mu_{x+j}+1-x-j<1-x$. Si $\mu_{j}=0$, on a $x+j=1$ et ${ }^{t} \mu_{x+j}+1-x-j$ est le plus grand terme de $R_{t} \mu$. L'inégalité précédente implique que $1-x$ n'intervient pas dans $R_{t} \mu$, c'est-à-dire mult $_{R_{t}}(1-x)=0$. Si $\mu_{j}>0$, on a $x+j-1 \geq 1$ et, d'après (1), l'égalité $x=\mu_{j}+1-j$ implique ${ }^{t} \mu_{x+j-1} \geq j$. Donc ${ }^{t} \mu_{x+j-1}+1-(x+j-1) \geq 2-x>1-x$. Alors $1-x$ est strictement compris entre deux termes consécutifs de $R_{t} \mu$ et n'intervient donc pas dans cette suite. De nouveau, mult $_{R_{t_{\mu}}}(1-x)=0$. Supposons maintenant mult $_{R_{\mu}}(x)=0$. Soit $j$ le plus petit entier strictement positif tel que $x>\mu_{j}+1-j$. Alors $x+j-1>\mu_{j}$. Si $j \geq 2$,

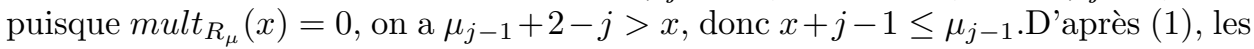
deux inégalités précédentes impliquent ${ }^{t} \mu_{x+j-1}=j-1$. On a supposé $j \geq 2$ mais le même résultat vaut si $j=1$ : l'inégalité $x+j-1>\mu_{j}=\mu_{1}$ entraîne $x+j-1 \geq 1$ et ${ }^{t} \mu_{x+j-1}=0=j-1$. On a alors en tout $\operatorname{cas}^{t} \mu_{x+j-1}+1-(x+j-1)=1-x$, donc mult $_{R_{t_{\mu}}}(1-x)=1$. Cela démontre $(2)$.

Pour tout $\mu=\left(\mu_{1}, \mu_{2}, \ldots\right) \in \mathcal{R}$ ou $\mu \in \mathcal{P}$, posons $2 \mu=\left(2 \mu_{1}, 2 \mu_{2}, \ldots\right)$ et $\mu^{2}=$ $\left(\mu_{1}, \mu_{1}, \mu_{2}, \mu_{2}, \mu_{3}, \ldots\right)$. Si $\mu \in \mathcal{P}$, on sait que ${ }^{t}(2 \mu)=\left({ }^{t} \mu\right)^{2}$.

Soit $(\lambda, \epsilon) \in \mathcal{P}^{s y m p}(2 N)$. Posons $k=k(\lambda, \epsilon)$. Il existe un unique élément de $\mathcal{P}^{s y m p}(2 N)$, que nous notons $\left({ }^{s} \lambda,{ }^{s} \epsilon\right)$ tel que $k\left({ }^{s} \lambda,{ }^{s} \epsilon\right)=k$ et $\rho\left({ }^{s} \lambda,{ }^{s} \epsilon\right)=\operatorname{sgn} \otimes$ $\rho(\lambda, \epsilon)$.

Remarque. Nous adoptons cette notation faute de mieux. Elle n'est pas très bonne car ${ }^{s} \lambda$ ne dépend pas seulement de $\lambda$ et ${ }^{s} \epsilon$ ne dépend pas seulement de $\epsilon$ : ils dépendent tous deux du couple $(\lambda, \epsilon)$.

Notons $(\alpha, \beta) \in \mathcal{P}_{2}(N-k(k+1) / 2)$ le couple paramétrant $\rho(\lambda, \epsilon)$. On sait que $\operatorname{sgn} \otimes \rho_{\alpha, \beta}=\rho^{t} \beta,{ }^{t} \alpha$. Donc $\left({ }^{t} \beta,{ }^{t} \alpha\right)$ est le couple paramétrant $\rho\left({ }^{s} \lambda,{ }^{s} \epsilon\right)$.

Lemme. On a l'égalité

$$
2 \Lambda_{k / 2,-(k+1) / 2 ; 1 / 2}(\alpha, \beta)={ }^{t}\left({ }^{s} \lambda\right)+\left(\left[0,-\infty\left[{ } _ { 1 } \sqcup \left[-1,-\infty\left[{ }_{1}\right) .\right.\right.\right.\right.
$$

Preuve. Posons $U=2 \alpha+\left[k,-\infty\left[{ }_{1}, V=2 \beta+\left[-k-1,-\infty\left[{ }_{1}, X=\left({ }^{t} \beta\right)^{2}+\left[k,-\infty\left[{ }_{1}\right.\right.\right.\right.\right.\right.$, $Y=\left({ }^{t} \alpha\right)^{2}+[-k-1,-\infty[1$. Les définitions entraînent que

$$
2 \Lambda_{k / 2,-(k+1) / 2 ; 1 / 2}(\alpha, \beta)=U \sqcup V .
$$

Pour $x \in \mathbb{Z}$, on a mult $_{U}(x)=$ mult $_{R_{2 \alpha}}(x-k)$ et mult $_{Y}(x)=$ mult $_{R_{\left(t_{\alpha}\right)^{2}}}(x+k+1)$. Grâce à (1), on en déduit

$$
\operatorname{mult}_{U}(x)+\operatorname{mult}_{Y}(-x)=1 .
$$


De même

$$
\operatorname{mult}_{V}(x)+\text { mult }_{X}(-x)=1 .
$$

Pour simplifier la notation, posons $(\mu, \tau)=\left({ }^{s} \lambda,{ }^{s} \epsilon\right)$. Pour tout $\nu=\left(\nu_{1}, \nu_{2}, \ldots\right) \in \mathcal{R}$, on pose $\nu-1=\left(\nu_{1}-1, \nu_{2}-1, \ldots\right)$. On se rappelle que

$$
p_{\mu, \tau}=\Lambda_{k,-k-1 ; 2}\left({ }^{t} \beta,{ }^{t} \alpha\right)=\left({ }^{t} \beta+\left[k,-\infty\left[{ }_{2}\right) \sqcup\left({ }^{t} \alpha+[-k-1,-\infty[2) .\right.\right.\right.
$$

Par construction, on a $X=\left({ }^{t} \beta+\left[k,-\infty[2) \sqcup\left({ }^{t} \beta+\left[k-1,-\infty[2), Y=\left({ }^{t} \alpha+[-k-\right.\right.\right.\right.\right.$ $1,-\infty\left[{ }_{2}\right) \sqcup\left({ }^{t} \alpha+\left[-k-2,-\infty[2)\right.\right.$. Alors $X \sqcup Y=p_{\mu, \tau} \sqcup\left(p_{\mu, \tau}-1\right)$. En rassemblant les égalités précédentes, on obtient

$$
\text { mult }_{2 \Lambda_{k / 2,-(k+1) / 2 ; 1 / 2}(\alpha, \beta)}(x)+\text { mult }_{p_{\mu, \tau} \sqcup\left(p_{\mu, \tau}-1\right)}(-x)=2 .
$$

Introduisons les partitions $\mu^{\prime}$ et $\mu^{\prime \prime}$ définies par $\mu_{j}^{\prime}=\left[\mu_{j} / 2\right], \mu_{j}^{\prime \prime}=\left[\left(\mu_{j}+1\right) / 2\right]$ pour $j \in \mathbb{N}_{>0}$. Montrons que, pour tout $x \in \mathbb{Z}$, on a

$$
p_{\mu, \tau} \sqcup\left(p_{\mu, \tau}-1\right)=R_{\mu^{\prime}} \sqcup\left(R_{\mu^{\prime \prime}}-1\right) .
$$

On a $p_{\mu, \tau}=A_{\mu}^{\sharp} \sqcup B_{\mu}^{\sharp}$ et ces suites sont calculées par les formules (1)(a) et (1)(b) de 4.1. On voit que les termes de toutes les suites intervenant dans (4) font intervenir un terme $\mu_{j}$ de la suite $\mu$. Il suffit d'analyser la contribution à ces suites de chaque terme $\mu_{j}$. Plus exactement, on analyse la contribution des termes $\mu_{j}$ pairs. Pour les termes impairs, puisque $\mu$ est symplectique, on peut les regrouper en paires de la forme $\mu_{2 j-1}=\mu_{2 j}$ avec $S_{2 j}(\mu)$ pair ou $\mu_{2 j}=\mu_{2 j+1}$ avec $S_{2 j+1}(\mu)$ pair et on analyse la contribution de chaque paire. Pour un indice $j \in \mathbb{N}_{>0}$ tel que $\mu_{j}$ est pair, $\mu_{j}$ contribue à $p_{\mu, \tau}$ par un terme $\mu_{j} / 2+1-j$, donc à $p_{\mu, \tau} \sqcup\left(p_{\mu, \tau}-1\right)$ par deux termes $\mu_{j} / 2+1-j$ et $\mu_{j} / 2-j$. Mais le premier, resp. le second, est la contribution de l'indice $j$ à $R_{\mu^{\prime}}$, resp. $R_{\mu^{\prime \prime}}-1$. Considérons un couple de termes impairs de la forme $\mu_{2 j-1}=\mu_{2 j}$, avec $S_{2 j}(\mu)$ pair. Ils contribuent à $p_{\mu, \tau}$ par les termes $A_{\mu, j}^{\sharp}$ et $B_{\mu, j}^{\sharp}$, tous deux égaux à $\left(\mu_{2 j}+1\right) / 2+1-2 j$. Ils contribuent à $p_{\mu, \tau} \sqcup\left(p_{\mu, \tau}-1\right)$ par quatre termes : deux fois $\left(\mu_{2 j}+1\right) / 2+1-2 j$ et deux fois $\left(\mu_{2 j}-1\right) / 2+1-2 j$. Les indices $2 j-1,2 j$ contribuent à $R_{\mu^{\prime}}$ par $\left(\mu_{2 j}+1\right) / 2+1-2 j$ et $\left(\mu_{2 j}-1\right) / 2+1-2 j$. Ils contribuent à $R_{\mu^{\prime \prime}}-1$ par les mêmes termes. Les contributions aux deux membres de (4) sont donc les mêmes. Considérons maintenant un couple de termes impairs de la forme $\mu_{2 j}=\mu_{2 j+1}$, avec $S_{2 j+1}(\mu)$ pair. Ils contribuent à $p_{\mu, \tau}$ par les termes $A_{\mu, j+1}^{\sharp}$ et $B_{\mu, j}^{\sharp}$, tous deux égaux à $\left(\mu_{2 j}+1\right) / 2-2 j$. Ils contribuent à $p_{\mu, \tau} \sqcup\left(p_{\mu, \tau}-1\right)$ par quatre termes : deux fois $\left(\mu_{2 j}+1\right) / 2-2 j$ et deux fois $\left(\mu_{2 j}-1\right) / 2-2 j$. Les indices $2 j, 2 j+1$ contribuent à $R_{\mu^{\prime}} \operatorname{par}\left(\mu_{2 j}+1\right) / 2-2 j$ et $\left(\mu_{2 j}-1\right) / 2-2 j$ et ils contribuent à $R_{\mu^{\prime \prime}}-1$ par les mêmes termes. Les contributions aux deux membres de (4) sont encore une fois les mêmes. Cela prouve (4).

D'après $(1)$, on a mult $R_{\mu^{\prime}}(x)+$ mult $_{R_{t_{\mu^{\prime}}-1}}(-x)=1$ et mult $_{R_{\mu^{\prime \prime}-1}}(x)+$ mult $_{R_{t_{\mu^{\prime \prime}}}}(-x)$ $=1$ pour tout $x \in \mathbb{Z}$. A l'aide de (3) et (4), on en déduit $\operatorname{mult}_{2 \Lambda_{k / 2,-(k+1) / 2 ; 1 / 2}(\alpha, \beta)}(x)=$ mult $_{R_{t_{\mu^{\prime \prime}}}}(x)+$ mult $_{R_{\mu_{\mu^{\prime}}-1}}(x)$, autrement dit

$$
2 \Lambda_{k / 2,-(k+1) / 2 ; 1 / 2}(\alpha, \beta)=R_{t} \mu^{\prime \prime} \sqcup\left(R_{t} \mu^{\prime}-1\right) .
$$

Montrons que

(6) pour tout $j \in \mathbb{N}_{>0}$, on a ${ }^{t} \mu^{\prime \prime}{ }_{j} \geq{ }^{t} \mu^{\prime}{ }_{j} \geq{ }^{t} \mu^{\prime \prime}{ }_{j+1}$.

Soit $h={ }^{t} \mu^{\prime}{ }_{j}$. Alors $\left[\mu_{h} / 2\right]=\mu_{h}^{\prime} \geq j$, donc aussi $\mu_{h}^{\prime \prime}=\left[\left(\mu_{h}+1\right) / 2\right] \geq j$ et ${ }^{t} \mu_{j}^{\prime \prime} \geq h$. Soit maintenant $h={ }^{t} \mu^{\prime \prime}{ }_{j+1}$. Alors $\left[\left(\mu_{h}+1\right) / 2\right]=\mu_{h}^{\prime \prime} \geq j+1$, donc aussi $\mu_{h}^{\prime}=\left[\mu_{h} / 2\right] \geq j$ et ${ }^{t} \mu_{j}^{\prime} \geq h$. Cela démontre (6). 
Il résulte de (6) que

$$
{ }^{t} \mu^{\prime} \sqcup^{t} \mu^{\prime \prime}=\left({ }^{t} \mu_{1}^{\prime \prime},{ }^{t} \mu^{\prime}{ }_{1},{ }^{t} \mu^{\prime \prime}{ }_{2}, \ldots\right) .
$$

et que

$$
\begin{gathered}
R_{t} \mu^{\prime \prime} \sqcup\left(R_{t} \mu^{\prime}-1\right)=\left({ }^{t} \mu^{\prime \prime}{ }_{1},{ }^{t} \mu^{\prime}{ }_{1}-1,{ }^{t} \mu^{\prime \prime}{ }_{2}-1,{ }^{t} \mu^{\prime}{ }_{2}-2, \ldots\right) \\
=\left({ }^{t} \mu^{\prime} \sqcup^{t} \mu^{\prime \prime}\right)+\left(\left[0,-\infty\left[{ } _ { 1 } \sqcup \left[-1,-\infty\left[{ }_{1}\right) .\right.\right.\right.\right.
\end{gathered}
$$

On sait que la transposition des partitions échange la somme et la réunion. Donc $\left({ }^{t} \mu^{\prime} \sqcup^{t} \mu^{\prime \prime}\right)={ }^{t}\left(\mu^{\prime}+\mu^{\prime \prime}\right)$. Mais, par définition, $\mu^{\prime}+\mu^{\prime \prime}=\mu$. D'où

$$
R_{t} \mu^{\prime \prime} \sqcup\left(R_{t} \mu^{\prime}-1\right)={ }^{t} \mu+\left(\left[0,-\infty\left[{ } _ { 1 } \sqcup \left[-1,-\infty\left[{ }_{1}\right) .\right.\right.\right.\right.
$$

Avec (5), on obtient l'énoncé.

\subsection{Un théorème de minimalité.}

Théorème. Soit $(\lambda, \epsilon) \in \mathcal{P}^{\text {symp }}(2 N)$. Supposons que $\lambda$ n'a que des termes pairs. Alors il existe un unique élément $\left(\lambda^{\text {min }}, \epsilon^{\text {min }}\right) \in \mathcal{P}^{s y m p}(2 N)$ vérifiant les propriétés suivantes :

(i) $\mathfrak{m u l t}\left(\lambda, \epsilon ;{ }^{s} \lambda^{\min },{ }^{s} \epsilon^{\min }\right)=1$;

(ii) pour tout élément $\left(\lambda^{\prime}, \epsilon^{\prime}\right) \in \mathcal{P}^{s y m p}(2 N)$ tel que $\mathfrak{m u l t}\left(\lambda, \epsilon{ }^{s} \lambda^{\prime},{ }^{s} \epsilon^{\prime}\right) \neq 0$, on a $\lambda^{\text {min }}<\lambda^{\prime}$ ou $\left(\lambda^{\prime}, \epsilon^{\prime}\right)=\left(\lambda^{\text {min }}, \epsilon^{\text {min }}\right)$.

De plus, on $a\left({ }^{s} \lambda^{\min },{ }^{s} \epsilon^{\min }\right)=\left(\lambda^{\max }, \epsilon^{\max }\right)$.

Preuve. On pose $k=k(\lambda, \epsilon)$. De nouveau, seuls interviennent les couples $\left(\lambda^{\prime}, \epsilon^{\prime}\right)$ tels que $k\left(\lambda^{\prime}, \epsilon^{\prime}\right)=k$, c'est-à-dire les éléments de $\mathcal{P}^{\text {symp }}(2 N)_{k}$. Soient $\left(\lambda^{\prime}, \epsilon^{\prime}\right),\left(\lambda^{\prime \prime}, \epsilon^{\prime \prime}\right) \in$ $\mathcal{P}^{\text {symp }}(2 N)_{k}$. Notons $\left(\alpha^{\prime}, \beta^{\prime}\right)$ et $\left(\alpha^{\prime \prime}, \beta^{\prime \prime}\right)$ les couples de partitions paramétrant $\rho\left(\lambda^{\prime}, \epsilon^{\prime}\right)$ et $\rho\left(\lambda^{\prime \prime}, \epsilon^{\prime \prime}\right)$. On a l'équivalence

(1) ${ }^{s} \lambda^{\prime} \leq s \lambda^{\prime \prime}$ si et seulement si $\Lambda_{k / 2,-(k+1) / 2 ; 1 / 2}\left(\alpha^{\prime \prime}, \beta^{\prime \prime}\right) \leq \Lambda_{k / 2,-(k+1) / 2 ; 1 / 2}\left(\alpha^{\prime}, \beta^{\prime}\right)$.

En effet, ${ }^{s} \lambda^{\prime} \leq{ }^{s} \lambda^{\prime \prime}$ équivaut à ${ }^{t}\left({ }^{s} \lambda^{\prime \prime}\right) \leq{ }^{t}\left({ }^{s} \lambda^{\prime}\right)$ et l'assertion résulte des lemmes

\section{6 et 1.1}

On peut reformuler la première assertion de l'énoncé sous la forme

il existe un unique élément $(\underline{\lambda}, \underline{\epsilon}) \in \mathcal{P}^{\operatorname{symp}}(2 N)_{k}$ vérifiant les propriétés

(i) $\mathfrak{m u l t}(\lambda, \epsilon ; \underline{\lambda}, \underline{\epsilon})=1$;

(ii) pour tout $\left(\lambda^{\prime}, \epsilon^{\prime}\right) \in \mathcal{P}^{s y m p}(2 N)_{k}$ tel que $\mathfrak{m u l t}\left(\lambda, \epsilon ; \lambda^{\prime}, \epsilon^{\prime}\right) \neq 0$, on a ${ }^{s} \underline{\lambda}<{ }^{s} \lambda^{\prime}$ ou $\left(\lambda^{\prime}, \epsilon^{\prime}\right)=(\underline{\lambda}, \underline{\epsilon})$.

Le couple $\left(\lambda^{\text {min }}, \epsilon^{\text {min }}\right)$ de l'énoncé est alors $\left({ }^{s} \underline{\lambda},{ }^{s} \underline{\epsilon}\right)$. La dernière assertion de l'énoncé affirme que $(\underline{\lambda}, \underline{\epsilon})=\left(\lambda^{\max }, \epsilon^{\max }\right)$.

L'assertion (1) et la proposition 4.2 permet de reformuler encore les assertions ci-dessus sous la forme suivante :

il existe un unique couple $(\underline{\alpha}, \underline{\beta}) \in \mathcal{P} \times \mathcal{P}$ vérifiant les conditions

(i) $\operatorname{mult}\left(\alpha, \beta,<_{\alpha, \beta, k} ; \underline{\alpha}, \underline{\beta}\right)=1$;

(ii) pour tout couple $\left(\alpha^{\prime}, \beta^{\prime}\right) \in \mathcal{P} \times \mathcal{P}$ tel que $\operatorname{mult}\left(\alpha, \beta,<_{\alpha, \beta, k} ; \alpha^{\prime}, \beta^{\prime}\right) \neq 0$, on a $\Lambda_{k / 2,-(k+1) / 2 ; 1 / 2}\left(\alpha^{\prime}, \beta^{\prime}\right)<\Lambda_{k / 2,-(k+1) / 2 ; 1 / 2}(\underline{\alpha}, \underline{\beta})$ ou $\left(\alpha^{\prime}, \beta^{\prime}\right)=(\underline{\alpha}, \underline{\beta})$.

La proposition 3.1 entraîne que ceci est vérifié si et seulement si l'ensemble $P_{k / 2,-(1+k) / 2 ; 1 / 2}\left(\alpha, \beta,<_{\alpha, \beta, k}\right)$ a un unique élément et, dans ce cas, $(\underline{\alpha}, \underline{\beta})$ est cet unique élément. On veut de plus prouver que cet élément est égal à l'élément $\left(\alpha^{\max }, \beta^{\max }\right)$ paramétrant $\left(\lambda^{\max }, \epsilon^{\max }\right)$. Dans la preuve du théorème 4.5 , on a 
vu que $\left(\alpha^{\max }, \beta^{\max }\right)$ était l'unique élément de $P_{k,-k-1 ; 2}\left(\alpha, \beta,<_{\alpha, \beta, k}\right)$. Il nous suffit donc de prouver que

$$
P_{k / 2,-(k+1) / 2 ; 1 / 2}\left(\alpha, \beta,<_{\alpha, \beta, k}\right)=P_{k,-k-1 ; 2}\left(\alpha, \beta,<_{\alpha, \beta, k}\right) .
$$

Or c'est ce qu'affirme le lemme 2.12, Cela achève la démonstration.

\section{Calcul explicite du Couple $\left(\lambda^{\max }, \epsilon^{\max }\right)$}

5.1. Définition d'un couple $(\bar{\lambda}, \bar{\epsilon})$. Soit $(\lambda, \epsilon) \in \mathcal{P}^{\operatorname{symp}}(2 N)$. On suppose que tous les termes de $\lambda$ sont pairs. On va associer par récurrence à $(\lambda, \epsilon)$ un autre couple $(\bar{\lambda}, \bar{\epsilon}) \in \mathcal{P}^{\text {symp }}(2 N)$.

Si $N=0,(\bar{\lambda}, \bar{\epsilon})=(\lambda, \epsilon)$ est l'unique élément de $\mathcal{P}^{s y m p}(0)$.

On suppose $N>0$. Comme en 4.1 on considère que $\epsilon$ est défini soit sur $\operatorname{Jord}^{b p}(\lambda)=\operatorname{Jord}(\lambda)$, soit sur l'ensemble d'indices $\mathbb{N}_{>0}: \epsilon(j)=\epsilon_{\lambda_{j}}$ si $\lambda_{j}>0$ et $\epsilon(j)=1$ si $\lambda_{j}=0$. Pour $\eta= \pm 1$, notons $J^{\eta}=\left\{j \in \mathbb{N}_{>0} ;(-1)^{j+1} \epsilon(j)=\eta\right\}$. Notons $\mathfrak{S}$ la réunion de $\{1\}$ et de l'ensemble des $j \geq 2$ tels que $\epsilon(j)(-1)^{j+1} \neq \epsilon(j-1)(-1)^{j}$. Remarquons que le complémentaire de $\mathfrak{S}$ dans $\mathbb{N}_{>0}$ est fini : on a $j \in \mathfrak{S}$ dès que $j \geq t(\lambda)+2$. On note $\tilde{J}^{\eta}$ l'ensemble des $j \in J^{\eta}$ tels que $j \notin \mathfrak{S}$. Posons

$$
\bar{\lambda}_{1}=\left(\sum_{s \in \mathfrak{S}} \lambda_{s}\right)-2\left|\tilde{J}^{-\epsilon(1)}\right| .
$$

Cet entier est pair et inférieur ou égal à $2 N$. Nous montrerons que

(1) on a $2 \leq \bar{\lambda}_{1}$

Posons $N^{\prime}=N-\bar{\lambda}_{1} / 2$. Notons $\lambda^{\prime}$ la réunion des $\lambda_{j}$ pour $j \in \tilde{J}^{\epsilon(1)}$ et des $\lambda_{j}+2$ pour $j \in \tilde{J}^{-\epsilon(1)}$. Les termes de cette partition sont tous pairs. Soit $i \in \operatorname{Jord}^{b p}\left(\lambda^{\prime}\right)$. Par définition, on peut fixer ou bien $j \in \tilde{J}^{\epsilon(1)}$ tel que $i=\lambda_{j}$, ou bien $j \in \tilde{J}^{-\epsilon(1)}$ tel que $i=\lambda_{j}+2$ (un tel $j$ n'est pas forcément unique mais on en fixe un). On pose $\epsilon_{i}^{\prime}=\epsilon(j)$ si $j \in \tilde{J}^{\epsilon(1)}, \epsilon_{i}^{\prime}=-\epsilon(j)$ si $j \in \tilde{J}^{-\epsilon(1)}$. Nous montrerons que

(2) cette définition ne dépend pas du choix de $j$;

(3) le couple $\left(\lambda^{\prime}, \epsilon^{\prime}\right)$ ainsi défini appartient à $\mathcal{P}^{\boldsymbol{s y m} \boldsymbol{m} \boldsymbol{p}}\left(2 N^{\prime}\right)$.

Puisque $N^{\prime}<N$ d'après (1), on sait par récurrence associer à $\left(\lambda^{\prime}, \epsilon^{\prime}\right)$ un couple $\left(\bar{\lambda}^{\prime}, \bar{\epsilon}^{\prime}\right) \in \mathcal{P}^{s y m p}\left(2 N^{\prime}\right)$. Nous montrerons que

(4) $\bar{\lambda}_{1} \geq \bar{\lambda}_{1}^{\prime}$.

On note $\bar{\lambda}$ la partition $\left\{\bar{\lambda}_{1}\right\} \sqcup \bar{\lambda}^{\prime}$. Elle appartient à $\mathcal{P}^{s y m p}(2 N)$. Nous montrerons que

(5) il existe un unique $\bar{\epsilon} \in\{ \pm 1\}^{\operatorname{Jord}^{b p}(\bar{\lambda})}$ tel que, pour $i \in \operatorname{Jord}^{b p}\left(\bar{\lambda}^{\prime}\right)$, on ait $\bar{\epsilon}_{i}=\bar{\epsilon}_{i}^{\prime}$ et que $\bar{\epsilon}_{\bar{\lambda}_{1}}=\epsilon(1)$.

On a ainsi défini le couple $(\bar{\lambda}, \bar{\epsilon}) \in \mathcal{P}^{s y m p}(2 N)$. On reporte à 5.2 les démonstrations des assertions (1) à (5).

Théorème. Le couple $\left(\lambda^{\max }, \epsilon^{\max }\right)$ intervenant dans le théorème 4.5] est égal au couple $(\bar{\lambda}, \bar{\epsilon})$ défini ci-dessus.

La démonstration sera donnée en 5.4 .

5.2. Démonstration des assertions de 5.1. On conserve les hypothèses et notations du paragraphe précédent en supposant $N>0$. On écrit $\mathfrak{S}=\left\{s_{1}, s_{2}, \ldots\right\}$. Remarquons que, si on décompose $J^{+1}$ et $J^{-1}$ en réunions d'intervalles non consécutifs, les éléments de $\mathfrak{S}$ sont exactement les plus petits termes de ces intervalles. Puisque $s_{1}=1 \in J^{\epsilon(1)}$, on a plus précisément

(1) $J^{\epsilon(1)}=\bigcup_{h \geq 1}\left\{s_{2 h-1}, \ldots, s_{2 h}-1\right\}, J^{-\epsilon(1)}=\bigcup_{h \geq 1}\left\{s_{2 h}, \ldots, s_{2 h+1}-1\right\}$. 
On a

(2) soient $\eta= \pm 1$ et $j \in \mathbb{N}_{>0}$; supposons $j, j+1 \in J^{\eta}$; alors $\lambda_{j} \geq \lambda_{j+1}+2$.

En effet, la fonction $j^{\prime} \mapsto \epsilon\left(j^{\prime}\right)(-1)^{j^{\prime}+1}$ est constante sur $J^{\eta}$. On en déduit $\epsilon(j)=-\epsilon(j+1)$. Cela interdit à $\lambda_{j}$ et $\lambda_{j+1}$ d'être égaux. L'assertion s'ensuit puisque ces termes sont pairs par hypothèse.

On en déduit

(3) pour $h \in \mathbb{N}_{>0}$, on a $\lambda_{s_{h}}-\lambda_{s_{h+1}} \geq 2\left(s_{h+1}-s_{h}-1\right)$.

C'est évident si $s_{h+1}=s_{h}+1$. Sinon, les termes $s_{h}, s_{h}+1, \ldots, s_{h+1}-1$ sont tous dans un même ensemble $J^{\eta}$. D'après (2), on a donc $\lambda_{s_{h}}-\lambda_{s_{h+1}-1} \geq 2\left(s_{h+1}-s_{h}-1\right)$. L'assertion s'ensuit puisque $\lambda_{s_{h+1}-1} \geq \lambda_{s_{h+1}}$.

En vertu de (1), la définition de $\bar{\lambda}_{1}$ donnée en 5.1 peut se récrire

$$
\begin{gathered}
\text { (4) } \quad \bar{\lambda}_{1}=\left(\sum_{h \geq 1} \lambda_{s_{h}}\right)-2 \sum_{h \geq 1}\left(s_{2 h+1}-s_{2 h}-1\right) \\
=\left(\sum_{h \geq 1} \lambda_{s_{2 h-1}}\right)+\left(\sum_{h \geq 1}\left(\lambda_{s_{2 h}}-2\left(s_{2 h+1}-s_{2 h}-1\right)\right)\right) .
\end{gathered}
$$

En vertu de (3), tous les termes du membre de droite ci-dessus sont positifs ou nuls. La première somme contient $\lambda_{1} \geq 2$. Donc $\bar{\lambda}_{1} \geq 2$. Cela prouve 5.1).

Par définition,

$$
S\left(\lambda^{\prime}\right)=\left(\sum_{j \in \mathbb{N}>0, j \notin \mathfrak{S}} \lambda_{j}\right)+2\left|\tilde{J}^{-\epsilon(1)}\right| .
$$

En comparant avec la définition initiale de $\bar{\lambda}_{1}$ donnée en 5.1] on voit que $\bar{\lambda}_{1}+S\left(\lambda^{\prime}\right)=$ $S(\lambda)=2 N$. Donc $S\left(\lambda^{\prime}\right)=2 N^{\prime}$.

Les termes de $\lambda^{\prime}$ sont des termes de $\lambda$ auxquels on ajoute éventuellement 2. Ces additions de 2 ne perturbent pas l'ordre des termes. Précisément,

(5) soient $\eta= \pm 1, j \in \tilde{J}^{\eta}$ et $k \in \tilde{J}^{-\eta}$; alors $j<k$ si et seulement si $\lambda_{j} \geq \lambda_{k}+2$.

Si $\lambda_{j} \geq \lambda_{k}+2$, on a a fortiori $\lambda_{j}>\lambda_{k}$ donc $j<k$. Inversement, supposons $j<k$. Soit $s$ le plus grand terme de la suite $\mathfrak{S}$ tel que $s<k$. D'après (1) et les hypothèses sur $j$ et $k$, on a $j<s$, donc $\lambda_{j} \geq \lambda_{s}$. D'après (2), on a $\lambda_{s} \geq \lambda_{k}+2$. D'où la conclusion.

Notons $r^{\prime}$ le nombre d'éléments de $\mathbb{N}_{>0}-\mathfrak{S}$. C'est la somme des nombres d'éléments de $\tilde{J}^{+1}$ et $\tilde{J}^{-1}$. Montrons que

(6) $\epsilon(1)=(-1)^{r^{\prime}}$.

On choisit un entier $R \geq 0$ tel que $j \in \mathfrak{S}$ pour tout $j \geq R+1$ et que $R \equiv$ $r^{\prime} \bmod 2 \mathbb{Z}$. L'intervalle $\{1, \ldots, R\}$ est réunion disjointe de $\tilde{J}^{+1}, \tilde{J}^{-1}$ et d'un sousensemble $\left\{s_{1}, \ldots, s_{S}\right\}$ de $\mathfrak{S}$. On a $R=S+r^{\prime}$, donc $S$ est pair. On a $R+1=s_{S+1}$. L'assertion (1) et la parité de $S$ entraînent que $R+1 \in J^{\epsilon(1)}$. D'autre part, pour deux éléments consécutifs $j$ et $j+1$ de $\mathfrak{S}$, on a $\epsilon(j)=\epsilon(j+1)$. Donc $\epsilon(R+1)=$ $\epsilon(R+2)=\ldots=1$. Les définitions impliquent que $R+1 \in J^{(-1)^{R}}=J^{(-1)^{r^{\prime}}}$. On a donc $J^{\epsilon(1)}=J^{(-1)^{r^{\prime}}}$, d'où $(6)$.

Notons $\varphi:\left\{1, \ldots, r^{\prime}\right\} \rightarrow \mathbb{N}_{>0}-\mathfrak{S}$ l'unique bijection croissante. En vertu de (5), la partition $\lambda^{\prime}$ se calcule de la façon suivante : pour $j \in\left\{1, \ldots, r^{\prime}\right\}$ tel que $\varphi(j) \in \tilde{J}^{\epsilon(1)}$, $\lambda_{j}^{\prime}=\lambda_{\varphi(j)}$; pour $j \in\left\{1, \ldots, r^{\prime}\right\}$ tel que $\varphi(j) \in \tilde{J}^{-\epsilon(1)}, \lambda_{j}^{\prime}=\lambda_{\varphi(j)}+2$; pour $j>r^{\prime}$, $\lambda_{j}^{\prime}=0$. Pour $\eta= \pm 1$, notons $J^{\prime} \eta$ la réunion de l'ensemble des $j \in\left\{1, \ldots, r^{\prime}\right\}$ tels que $\varphi(j) \in \tilde{J}^{-\eta}$ et de l'ensemble des $j>r^{\prime}$ tels que $(-1)^{j+1}=\eta$. Définissons une fonction $\epsilon^{\prime}$ sur $\mathbb{N}_{>0}$ par 
pour $\eta= \pm 1$ et $j \in J^{\prime} \eta \cap\left\{1, \ldots, r^{\prime}\right\}, \epsilon^{\prime}(j)=-\eta \epsilon(1) \epsilon(\varphi(j))$;

pour $j>r^{\prime}, \epsilon^{\prime}(j)=1$.

La notation $\epsilon^{\prime}$ anticipe le résultat suivant :

(7) pour $j, k \in \mathbb{N}_{>0}$ tels que $\lambda_{j}^{\prime}=\lambda_{k}^{\prime}$, on a $\epsilon^{\prime}(j)=\epsilon^{\prime}(k)$; pour $j \in \mathbb{N}_{>0}$ tel que $\lambda_{j}^{\prime}=0$, on a $\epsilon^{\prime}(j)=1$.

Si $j>r^{\prime}$, on a $\lambda_{j}^{\prime}=0$ et $\epsilon^{\prime}(j)=1$. Supposons $j \leq r^{\prime}$ et $\lambda_{j}^{\prime}=0$. Alors $\varphi(j) \in \tilde{J}^{\epsilon(1)}$ $\left(\operatorname{sinon} \lambda_{j}^{\prime}=\lambda_{\varphi(j)}+2 \geq 2\right.$ ) et $\lambda_{\varphi(j)}=\lambda_{j}^{\prime}=0$. Puisque $\lambda_{\varphi(j)}=0$, on a $\epsilon(\varphi(j))=1$. Alors la définition implique $\epsilon^{\prime}(j)=1$, ce qui démontre la dernière assertion de $(7)$. Cela étant démontré, on peut supposer pour la première assertion que $\lambda_{j}^{\prime}=\lambda_{k}^{\prime}>0$, donc $j, k \leq r^{\prime}$. Il suffit de traiter le cas où $k=j+1$. Si $\varphi(j)$ et $\varphi(j+1)$ appartiennent à un même ensemble $\tilde{J}^{\eta}$, on a $\lambda_{j}^{\prime}=\lambda_{\varphi(j)}+c, \lambda_{j+1}^{\prime}=\lambda_{\varphi(j+1)}+c$ pour un même nombre $c=0$ ou 2 , et $\lambda_{\varphi(j)} \geq \lambda_{\varphi(j+1)}+2$ d'après (2). Donc $\lambda_{j}^{\prime}>\lambda_{j+1}^{\prime}$ contrairement à l'hypothèse. Donc $\varphi(j)$ et $\varphi(j+1)$ n'appartiennent pas à un même ensemble $\tilde{J}^{\eta}$ et sont séparés par au moins un élément de $\mathfrak{S}$. D'autre part, $\varphi(j)$ et $\varphi(j+1)$ sont deux termes consécutifs de l'ensemble $\mathbb{N}_{>0}-\mathfrak{S}$. Autrement dit l'ensemble $\{\varphi(j)+1, \ldots, \varphi(j+1)-1\}$ est non vide et est contenu dans $\mathfrak{S}$. Les indices $\varphi(j+1)-1$ et $\varphi(j+1)$ appartiennent à un même ensemble $J^{\eta}$. Donc $\lambda_{\varphi(j+1)-1} \geq \lambda_{\varphi(j+1)}+2$ d'après (2). On a alors

$$
\lambda_{j}^{\prime} \geq \lambda_{\varphi(j)} \geq \lambda_{\varphi(j)+1} \geq \ldots \geq \lambda_{\varphi(j+1)-1} \geq \lambda_{\varphi(j+1)}+2 \geq \lambda_{j+1}^{\prime} .
$$

Les termes extrêmes étant égaux, ces inégalités sont des égalités. Les égalités extrêmes impliquent que $\varphi(j) \in \tilde{J}^{\epsilon(1)}$ et $\varphi(j+1) \in \tilde{J}^{-\epsilon(1)}$. Par définition, on a $\epsilon^{\prime}(j)=\epsilon(\varphi(j))$ et $\epsilon_{j+1}^{\prime}=-\epsilon(\varphi(j+1))$. On a $\epsilon(\varphi(j))=\epsilon(\varphi(j+1)-1)$ puisque $\lambda_{\varphi(j)}=\lambda_{\varphi(j+1)-1}$ d'après les égalités ci-dessus. On a aussi $\epsilon(\varphi(j+1)-1)=$ $-\epsilon(\varphi(j+1))$ puisque $\varphi(j+1)-1$ et $\varphi(j+1)$ sont deux termes consécutifs de $J^{-\epsilon(1)}$. On conclut $\epsilon^{\prime}(j)=\epsilon^{\prime}(j+1)$, ce qui démontre $(7)$.

Cette assertion entraîne que la fonction $\epsilon^{\prime}$ définie sur $\mathbb{N}_{>0}$ provient d'une fonction $\epsilon^{\prime}$ définie sur $J_{o r d} d^{b p}\left(\lambda^{\prime}\right)$. En comparant, les définitions, on voit que c'est la fonction définie en 5.1. Cela entraîne les deux assertions (2) et (3) de ce paragraphe.

Remarquons que les définitions des ensembles $J^{\prime} \eta$ pour $\eta= \pm 1$ sont cohérentes avec celles de 5.1. C'est-à-dire que

(8) pour $\eta= \pm 1$ et $j \in J^{\prime} \eta$, on a $(-1)^{j+1} \epsilon^{\prime}(j)=\eta$.

Si $j>r^{\prime}$, c'est immédiat. Supposons $j \leq r^{\prime}$. Notons $f(j)$ le plus grand entier strictement positif tel que $s_{f(j)}<\varphi(j)$. Alors $\varphi(j)=j+f(j)$. Par définition, $\varphi(j) \in \tilde{J}^{-\eta}$ donc $\epsilon(\varphi(j))=\eta(-1)^{\varphi(j)}=\eta(-1)^{j+f(j)}$. Il résulte de $(1)$ que $f(j)$ est impair si $\eta=-\epsilon(1)$ et $f(j)$ est pair si $\eta=\epsilon(1)$. Donc $\epsilon(\varphi(j))=\epsilon(1)(-1)^{j}$. Alors $\epsilon^{\prime}(j)=-\eta \epsilon(1) \epsilon(\varphi(j))=(-1)^{j+1} \eta$, ce qui démontre (8).

Montrons que

(9) on a $\bar{\lambda}_{1} \geq \bar{\lambda}_{1}^{\prime}$; si $\bar{\lambda}_{1}=\bar{\lambda}_{1}^{\prime}$, on a $\bar{\epsilon}_{\bar{\lambda}_{1}^{\prime}}^{\prime}=\epsilon(1)$.

L'assertion est triviale si $N^{\prime}=0$ puisqu'alors $\bar{\lambda}_{1}^{\prime}=0<\bar{\lambda}_{1}$ d'après [5.1(1). On suppose $N^{\prime}>0$. Remarquons que, puisque $\bar{\epsilon}_{\bar{\lambda}_{1}^{\prime}}^{\prime}=\epsilon^{\prime}(1)$ par définition, l'assertion (9) peut se reformuler ainsi : si $\epsilon^{\prime}(1)=\epsilon(1)$, on a $\bar{\lambda}_{1} \geq \bar{\lambda}_{1}^{\prime}$; si $\epsilon^{\prime}(1) \neq \epsilon(1)$, on a $\bar{\lambda}_{1}>\bar{\lambda}_{1}^{\prime}$.

On introduit pour le couple $\left(\lambda^{\prime}, \epsilon^{\prime}\right)$ les objets similaires à ceux introduits en [5.1]: la suite $\mathfrak{S}^{\prime}=\left(s_{1}^{\prime}, s_{2}^{\prime}, \ldots\right)$ et les ensembles $\tilde{J}^{\prime} \eta$ pour $\eta= \pm 1$. On a remarqué en 5.1 que $j \in \mathfrak{S}^{\prime}$ si $j \geq t\left(\lambda^{\prime}\right)+2$ a fortiori si $j \geq r^{\prime}+2$. L'indice $r^{\prime}+1$ appartient à $J^{\prime}(-1)^{r^{\prime}}$, c'est-à-dire $J^{\prime} \epsilon(1)$ d'après (6). Il appartient à $\mathfrak{S}^{\prime}$ si et seulement si $r^{\prime}$ appartient à $J^{\prime}-\epsilon(1)$. Notons $S^{\prime}$ le plus grand indice $h \geq 1$ tel que $s_{h}^{\prime} \leq r^{\prime}$. Pour $h^{\prime} \in\left\{1, \ldots, S^{\prime}\right\}$, 
posons $\psi\left(h^{\prime}\right)=f\left(s_{h^{\prime}}^{\prime}\right)$. Autrement dit $\psi\left(h^{\prime}\right)$ est le plus grand indice $h \geq 1$ tel que $s_{h}<\varphi\left(s_{h^{\prime}}^{\prime}\right)$. Montrons que

(10) l'application $\psi$ est strictement croissante; pour $h^{\prime} \in\left\{1, \ldots, S^{\prime}\right\}$, on a :

$\varphi\left(s_{h^{\prime}}^{\prime}\right)=s_{\psi\left(h^{\prime}\right)}+1$;

$\lambda_{s_{h^{\prime}}^{\prime}}^{\prime} \leq \lambda_{s_{\psi\left(h^{\prime}\right)}}$ et $\lambda_{s_{h^{\prime}}^{\prime}}^{\prime} \leq \lambda_{s_{\psi\left(h^{\prime}\right)}}-2$ si $s_{h^{\prime}}^{\prime} \in J^{\prime}-\epsilon(1)$

$h^{\prime} \equiv \psi\left(h^{\prime}\right) \bmod 2 \mathbb{Z}^{h^{\prime}}$ si $\epsilon^{\prime}(1) \epsilon(1)=-1$ et $h^{\prime} \equiv \psi\left(h^{\prime}\right)+1 \bmod 2 \mathbb{Z}$ si $\epsilon^{\prime}(1) \epsilon(1)=1$.

Soit $\eta= \pm 1$ tel que $s_{h^{\prime}}^{\prime} \in J^{\prime} \eta$. Alors $\varphi\left(s_{h^{\prime}}^{\prime}\right) \in J^{-\eta}$. D'après la définition, l'intervalle $\left\{s_{\psi\left(h^{\prime}\right)}, s_{\psi\left(h^{\prime}\right)}+1, \ldots, \varphi\left(s_{h^{\prime}}^{\prime}\right)\right\}$ est contenu dans $J^{-\eta}$. S'il a au moins trois éléments, $\varphi\left(s_{h^{\prime}}^{\prime}\right)-1$ est forcément égal à $\varphi\left(s_{h^{\prime}}^{\prime}-1\right)$ et alors $s_{h^{\prime}}^{\prime}-1$ appartient à $J^{\prime \eta}$. C'est impossible par définition de la suite $\mathfrak{S}^{\prime}$ : les éléments $s_{h^{\prime}}^{\prime}-1$ et $s_{h^{\prime}}^{\prime}$ ne sont pas dans un même ensemble $J^{\prime} \eta$. Donc l'intervalle ci-dessus n'a que deux éléments, c'est-à-dire $\varphi\left(s_{h^{\prime}}^{\prime}\right)=s_{\psi\left(h^{\prime}\right)}+1$. D'après (2), on a $\lambda_{s_{\psi\left(h^{\prime}\right)}} \geq \lambda_{s_{\psi\left(h^{\prime}\right)}+1}+2=$ $\lambda_{\varphi\left(s_{h^{\prime}}^{\prime}\right)}+2 \geq \lambda_{s_{h^{\prime}}^{\prime}}^{\prime}$. La dernière inégalité se renforce en $\lambda_{\varphi\left(s_{h^{\prime}}^{\prime}\right)}+2=\lambda_{s_{h^{\prime}}^{\prime}}^{\prime}+2$ si $\varphi\left(s_{h^{\prime}}^{\prime}\right) \in J^{\epsilon(1)}$ c'est-à-dire si $s_{h^{\prime}}^{\prime} \in J^{\prime-\epsilon(1)}$. Appliquons (1) : puisque $s_{h^{\prime}}^{\prime} \in J^{\prime} \eta, h^{\prime}$ est impair si et seulement si $\eta=\epsilon^{\prime}(1)$; puisque $s_{\psi\left(h^{\prime}\right)} \in J^{-\eta}, \psi\left(h^{\prime}\right)$ est impair si et seulement si $-\eta=\epsilon(1)$. On en déduit les congruences annoncées. L'application $\psi$ est croissante par construction. Les congruences précédentes entraînent que $\psi\left(h^{\prime}+1\right)$ n'est pas de la même parité que $\psi\left(h^{\prime}\right)$. Donc $\psi$ est strictement croissante. Cela démontre (10).

Posons $\tilde{\mathfrak{S}}^{\prime}=\left\{1, \ldots, r^{\prime}\right\} \cap \mathfrak{S}^{\prime}=\left\{s_{1}^{\prime}, \ldots, s_{S^{\prime}}^{\prime}\right\}$. Puisque $\lambda_{j}^{\prime}=0$ si $j \geq r^{\prime}+1$, il résulte de (10) que

$$
\sum_{s^{\prime} \in \mathfrak{S}^{\prime}} \lambda_{s^{\prime}}^{\prime} \leq \sum_{h^{\prime}=1, \ldots, S^{\prime}} \lambda_{s_{h^{\prime}}^{\prime}}^{\prime} \leq \sum_{h \in \operatorname{Im}(\psi)} \lambda_{s_{h}}-2\left|\tilde{\mathfrak{S}}^{\prime} \cap J^{\prime-\epsilon(1)}\right|,
$$

où $\operatorname{Im}(\psi)$ est l'image de $\psi$. En appliquant les définitions de $\bar{\lambda}_{1}$ et $\bar{\lambda}_{1}^{\prime}$ de 5.1, on obtient

$$
\text { (11) } \quad \bar{\lambda}_{1}^{\prime} \leq \bar{\lambda}_{1}-X
$$

où

$$
X=\left(\sum_{h \in \mathbb{N}_{>0}-\operatorname{Im}(\psi)} \lambda_{s_{h}}\right)+2\left|\tilde{\mathfrak{S}}^{\prime} \cap J^{\prime-\epsilon(1)}\right|+2\left|\tilde{J}^{\prime}-\epsilon^{\prime}(1)\right|-2\left|\tilde{J}^{-\epsilon(1)}\right| .
$$

Supposons $\epsilon^{\prime}(1)=\epsilon(1)$. D'après (10), le plus petit terme $\psi(1)$ de $\operatorname{Im}(\psi)$ est pair. Donc $1 \notin \operatorname{Im}(\psi)$ et

$$
X \geq \lambda_{s_{1}}-2\left|\tilde{J}^{-\epsilon(1)}\right|
$$

On a

$$
\lambda_{s_{1}}=\sum_{h \in \mathbb{N}_{>0}}\left(\lambda_{s_{h}}-\lambda_{s_{h+1}}\right) \geq \sum_{h \in \mathbb{N}_{>0}}\left(\lambda_{s_{2 h}}-\lambda_{s_{2 h+1}}\right) .
$$

Grâce à (3), on obtient

$$
\lambda_{s_{1}} \geq 2 \sum_{h \in \mathbb{N}_{>0}}\left(s_{2 h+1}-s_{2 h}-1\right) .
$$

Or, d'après $(1)$, cette dernière somme vaut $\left|\tilde{J}^{-\epsilon(1)}\right|$. On en déduit $X \geq 0$, donc $\bar{\lambda}_{1}^{\prime} \leq \bar{\lambda}_{1}$ d'après $(11)$.

Supposons maintenant $\epsilon^{\prime}(1)=-\epsilon(1)$. D'après (11), on doit prouver que $X \geq 2$. D'après les définitions, $\varphi$ se restreint en une bijection de $J^{\prime} \epsilon(1) \cap\left\{1, \ldots, r^{\prime}\right\}$ sur $\tilde{J}^{-\epsilon(1)}$. L'ensemble $J^{\prime} \epsilon(1) \cap\left\{1, \ldots, r^{\prime}\right\}$ est réunion disjointe de $\tilde{\mathfrak{S}}^{\prime} \cap J^{\prime} \epsilon(1)$ et de $\tilde{J}^{\prime} \epsilon(1) \cap$ 
$\left\{1, \ldots, r^{\prime}\right\}$. De plus, $\tilde{J}^{\prime} \epsilon(1)$ est réunion disjointe de $\tilde{J}^{\prime} \epsilon(1) \cap\left\{1, \ldots, r^{\prime}\right\}$ et de $\tilde{J}^{\prime} \epsilon(1) \cap$ $\left\{r^{\prime}+1\right\}$, puisque $j \in \mathfrak{S}^{\prime}$ si $j \geq r^{\prime}+2$. On obtient

$$
\left|\tilde{J}^{-\epsilon(1)}\right|=\left|\tilde{\mathfrak{S}}^{\prime} \cap J^{\prime} \epsilon(1)\right|+\left|\tilde{J}^{\prime} \epsilon(1)\right|-\left|\tilde{J}^{\prime} \epsilon(1) \cap\left\{r^{\prime}+1\right\}\right| .
$$

En utilisant l'hypothèse $\epsilon^{\prime}(1)=-\epsilon(1)$, la définition de $X$ se récrit

$$
X=\left(\sum_{h \in \mathbb{N}>0-\operatorname{Im}(\psi)} \lambda_{s_{h}}\right)+2\left(\left|\tilde{\mathfrak{S}}^{\prime} \cap J^{\prime} \epsilon^{\prime}(1)\right|-2\left|\tilde{\mathfrak{S}}^{\prime} \cap J^{\prime}-\epsilon^{\prime}(1)\right|\right)+2\left|\tilde{J}^{\prime}-\epsilon^{\prime}(1) \cap\left\{r^{\prime}+1\right\}\right| .
$$

D'après (1), $\left|\tilde{\mathfrak{S}}^{\prime} \cap J^{\prime} \epsilon^{\prime}(1)\right|$ est le nombre de termes impairs dans l'intervalle $\left\{1, \ldots, S^{\prime}\right\}$ tandis que $\left|\tilde{\mathfrak{S}}^{\prime} \cap J^{\prime}-\epsilon^{\prime}(1)\right|$ est le nombre de termes pairs. Leur différence vaut 1 si $S^{\prime}$ est impair, 0 si $S^{\prime}$ est pair. L'expression (11) est alors somme de trois expressions positives ou nulles, donc $X \geq 0$. Si $S^{\prime}$ est impair, l'expression centrale vaut 2 donc $X \geq 2$ comme on le voulait. Supposons $S^{\prime}$ pair. Par définition de $S^{\prime}$, l'intervalle $\left\{s_{S^{\prime}}^{\prime}, \ldots, r^{\prime}\right\}$ est contenu dans un même intervalle $J^{\prime} \eta$. Puisque $S^{\prime}$ est pair, on a $s_{S^{\prime}}^{\prime} \in J^{\prime}-\epsilon^{\prime}(1)$ d'après (1), donc $r^{\prime} \in J^{\prime}-\epsilon^{\prime}(1)$. On a vu plus haut que $r^{\prime}+1 \epsilon$ $J^{\prime} \epsilon(1)=J^{\prime}-\epsilon^{\prime}(1)$. Alors $r^{\prime}+1$ n'appartient pas à $\mathfrak{S}^{\prime}$, donc $r^{\prime}+1 \in \tilde{J}^{\prime}-\epsilon^{\prime}(1)$. Le dernier terme de $(12)$ vaut 2 , donc $X \geq 2$. Cela achève la preuve de (9).

Cette assertion (9) implique l'assertion (5) de 5.1. Cela achève la vérification des propriétés énoncées dans ce paragraphe.

5.3. Conservation de l'entier $k$. Les hypothèses sont les mêmes qu'en 5.1.

Lemme. On a l'égalité $k(\lambda, \epsilon)=k(\bar{\lambda}, \bar{\epsilon})$.

Preuve. C'est trivial si $N=0$. On suppose $N>0$. Posons $M=M(\lambda, \epsilon), \bar{M}=$ $M(\bar{\lambda}, \bar{\epsilon})$, cf. 4.1. Rappelons que $k(\lambda, \epsilon)=\sup (2 M,-2 M-1), k(\bar{\lambda}, \bar{\epsilon})=\sup (2 \bar{M},-2 \bar{M}$ -1). L'égalité à démontrer équivaut à $\bar{M}=M$.

Pour $h \in \mathbb{Z}$, posons $\tau(h)=0$ si $h$ est pair, $\tau(h)=1$ si $h$ est impair. Soit $h \geq 0$ un entier tel que $\epsilon(j)=1$ pour tout $j \geq h+1$. Montrons que

(1) $2 M=\left|J^{1} \cap\{1, \ldots, h\}\right|-\left|J^{-1} \cap\{1, \ldots, h\}\right|-\tau(h)$.

On utilise la formule 4.1(2), que l'on peut tronquer à l'ordre $h$, les termes d'indices supérieurs étant nuls. On obtient

$$
2 M=\left(\sum_{j=1, \ldots, h}(-1)^{j+1} \epsilon(j)\right)-\left(\sum_{j=1, \ldots, h}(-1)^{j+1}\right) .
$$

En appliquant les définitions, on voit que la première somme vaut $\left|J^{1} \cap\{1, \ldots, h\}\right|-$ $\left|J^{-1} \cap\{1, \ldots, h\}\right|$. Le calcul de la seconde donne $\tau(h)$. D'où $(1)$.

Posons $M^{\prime}=M\left(\lambda^{\prime}, \epsilon^{\prime}\right)$. On va prouver

$$
\text { (2) } \quad M^{\prime}=\left\{\begin{array}{cc}
-M, & \text { si } \epsilon(1)=1, \\
-M-1, & \text { si } \epsilon(1)=-1 .
\end{array}\right.
$$

Fixons un entier $R$ tel que $R \equiv r^{\prime} \bmod 2 \mathbb{Z}$ et que $j \in \mathfrak{S}$ pour tout entier $j \geq$ $R+1$. L'intervalle $\{1, \ldots, R\}$ est réunion disjointe de $\tilde{J}^{1}, \tilde{J}^{-1}$ et d'un sous-ensemble $\left\{s_{1}, \ldots, s_{S}\right\}$ de $\mathfrak{S}$. On se rappelle que $r^{\prime}$ est la somme des nombres d'éléments de $\tilde{J}^{1}$ et de $\tilde{J}^{-1}$. La condition de congruence que l'on a imposée implique que $S$ est pair. D'autre part, par définition de $\mathfrak{S}$, on a $\epsilon(j)=\epsilon(j+1)$ si $j$ et $j+1$ appartiennent à S. Donc $\epsilon(j)=1$ pour tout $j \geq R+1$ et on peut appliquer la formule (1) avec $h=R$. On obtient

$$
2 M=\left|\tilde{J}^{1}\right|+\left|\left\{s_{1}, \ldots, s_{S}\right\} \cap J^{1}\right|-\left|\tilde{J}^{-1}\right|-\left|\left\{s_{1}, \ldots, s_{S}\right\} \cap J^{-1}\right|-\tau\left(r^{\prime}\right) .
$$


La relation $5.2(1)$ et la parité de $S$ entraînent que $\left|\left\{s_{1}, \ldots, s_{S}\right\} \cap J^{1}\right|=\mid\left\{s_{1}, \ldots, s_{S}\right\} \cap$ $J^{-1} \mid$. D'où

$$
\text { (3) } \quad 2 M=\left|\tilde{J}^{1}\right|-\left|\tilde{J}^{-1}\right|-\tau\left(r^{\prime}\right) \text {. }
$$

Pour calculer $M^{\prime}$, on utilise la formule analogue à (1) en prenant $h=r^{\prime}$. On obtient

$$
2 M^{\prime}=\left|J^{\prime 1} \cap\left\{1, \ldots, r^{\prime}\right\}\right|-\left|J^{\prime-1} \cap\left\{1, \ldots, r^{\prime}\right\}\right|-\tau\left(r^{\prime}\right) .
$$

Puisque $\varphi$ se restreint en des bijections de $J^{\prime} \eta \cap\left\{1, \ldots, r^{\prime}\right\}$ sur $\tilde{J}^{-\eta}$ pour $\eta= \pm 1$, cette égalité devient

$$
2 M^{\prime}=\left|\tilde{J}^{-1}\right|-\left|\tilde{J}^{-1}\right|-\tau\left(r^{\prime}\right) .
$$

L'assertion (2) résulte des deux formules ci-dessus et de l'assertion [5.2(6) qui nous dit que $\tau\left(r^{\prime}\right)=0$ si $\epsilon(1)=1, \tau\left(r^{\prime}\right)=1$ si $\epsilon(1)=-1$.

Posons $\bar{M}^{\prime}=M\left(\bar{\lambda}^{\prime}, \bar{\epsilon}^{\prime}\right)$. On va prouver

$$
\text { (4) } \quad \bar{M}^{\prime}=\left\{\begin{array}{cc}
-\bar{M}, & \text { si } \epsilon(1)=1, \\
-\bar{M}-1, & \text { si } \epsilon(1)=-1 .
\end{array}\right.
$$

Par définition, $\bar{\epsilon}(j)=\bar{\epsilon}^{\prime}(j-1)$ pour tout $j \geq 2$. La formule 4.1 (2) entraîne donc

$$
\begin{gathered}
2 \bar{M}=(\bar{\epsilon}(1)-1)+\sum_{j \geq 2}(-1)^{j+1}(\bar{\epsilon}(j)-1)=(\bar{\epsilon}(1)-1)-\sum_{j \geq 1}(-1)^{j+1}\left(\bar{\epsilon}^{\prime}(j)-1\right) \\
=(\bar{\epsilon}(1)-1)-2 \bar{M}^{\prime} .
\end{gathered}
$$

Puisque $\bar{\epsilon}(1)=\epsilon(1)$ par définition, on obtient (4).

On applique notre lemme par récurrence : on a $M^{\prime}=\bar{M}^{\prime}$. Alors les relations (2) et (4) entraînent $M=\bar{M}$.

Extrayons de la preuve ci-dessus deux formules qui nous seront utiles plus loin. Introduisons la notation suivante : pour $u \in\{ \pm 1\}$ et $h \in \mathbb{Z}$, posons $h^{u}=h$ si $u=1$ et $h^{u}=-h-1$ si $u=-1$ (on prendra garde à cette notation : si $u=-1, h^{u}$ n'est pas l'inverse de $h)$. Posons $k=k(\lambda, \epsilon), k^{\prime}=k\left(\lambda^{\prime}, \epsilon^{\prime}\right), v=\epsilon(1)(-1)^{k}, v^{\prime}=\epsilon(1)(-1)^{k^{\prime}+1}$. Montrons que

(5) $k^{\prime} v^{\prime}=k^{v}-1$;

(6) $k^{v}=\left|\tilde{J}^{\epsilon(1)}\right|-\left|\tilde{J}^{-\epsilon(1)}\right|$.

La définition $k=\sup (2 M,-2 M-1)$ équivaut à $k^{(-1)^{k}}=2 M$. On a alors $k^{v}=$ $(2 M)^{\epsilon(1)}$ et, de même, $k^{\prime} v^{\prime}=\left(2 M^{\prime}\right)^{-\epsilon(1)}$. Or l'égalité (2) équivaut à $\left(2 M^{\prime}\right)^{-\epsilon(1)}=$ $(2 M)^{\epsilon(1)}-1$. D'où (5). On vient de voir que $k^{v}=(2 M)^{\epsilon(1)}$. La relation (3) entraîne que ceci vaut $\left|\tilde{J}^{\epsilon(1)}\right|-\left|\tilde{J}^{-\epsilon(1)}\right|-\tau\left(r^{\prime}\right)$ si $\epsilon(1)=1,\left|\tilde{J}^{\epsilon(1)}\right|-\left|\tilde{J}^{-\epsilon(1)}\right|+\tau\left(r^{\prime}\right)-1$ si $\epsilon(1)=-1$. Or $\tau\left(r^{\prime}\right)=0$ si $\epsilon(1)=1, \tau\left(r^{\prime}\right)=1$ si $\epsilon(1)=-1$. D'où $(6)$.

5.4. Preuve du théorème [5.1. Les calculs de cette preuve diffèrent selon les différents cas $\epsilon(1)= \pm 1$ et $k$ pair ou impair. Pour tenter de les unifier, reprenons les définitions de 4.1 .

Soit $u \in\{ \pm 1\}$. Pour tout couple $(\lambda, \mu) \in \mathcal{R} \times \mathcal{R}$ ou $(\lambda, \mu) \in \mathcal{P} \times \mathcal{P}$, posons $\left(\lambda^{u}, \mu^{u}\right)=(\lambda, \mu)$ si $u=1,\left(\lambda^{u}, \mu^{u}\right)=(\mu, \lambda)$ si $u=-1$. Pour tout entier $h \in \mathbb{Z}$, posons comme dans le paragraphe précédent $h^{u}=h$ si $u=1$ et $h^{u}=-h-1$ si $u=-1$. Pour $(\lambda, \epsilon) \in \mathcal{P}^{s y m p}(2 N)$ et $(\alpha, \beta) \in \mathcal{P} \times \mathcal{P}$, le fait que $(\alpha, \beta)$ paramètre la représentation $\rho(\lambda, \epsilon)$ équivaut aux égalités

$$
A_{\lambda, \epsilon}^{u}=\alpha^{v}+\left[k^{v},-\infty\left[{ }_{2} B_{\lambda, \epsilon}^{u}=\beta^{v}+\left[-1-k^{v},-\infty\left[{ }_{2},\right.\right.\right.\right.
$$

où $k=k(\lambda, \epsilon)$ et $v=u(-1)^{k}$. 
Soit $N \in \mathbb{N}_{>0}$. Fixons $(\lambda, \epsilon) \in \mathcal{P}^{\text {symp }}(2 N)$ et supposons que tous les termes de $\lambda$ sont pairs. On pose $k=k(\lambda, \epsilon)$. Soit $(\alpha, \beta) \in \mathcal{P}_{2}(N-k(k+1) / 2)$ le couple paramétrant $\rho(\lambda, \epsilon)$. On introduit les mêmes objets $J^{+1}, J^{-1}$, $\mathfrak{S}$ etc... qu'en [5.1. On a calculé les suites $A_{\lambda, \epsilon}$ et $B_{\lambda, \epsilon}$ en 4.1. On en déduit les égalités

$$
A_{\lambda, \epsilon}^{u}=\left\{\lambda_{j} / 2+1-j ; j \in J^{u}\right\}, B_{\lambda, \epsilon}^{u}=\left\{\lambda_{j} / 2+1-j ; j \in J^{-u}\right\} .
$$

Considérons d'abord le cas particulier où $N=k(k+1) / 2$. Le couple $(\alpha, \beta)$ est forcément $(\emptyset, \emptyset)$, donc $(\lambda, \epsilon)$ est uniquement déterminé. C'est-à-dire que l'ensemble $\mathcal{P}^{\operatorname{symp}}(2 N)_{k}$ est réduit à cet unique couple. Or $\left(\lambda^{\max }, \epsilon^{\max }\right)$ et $(\bar{\lambda}, \bar{\epsilon})$ appartiennent tous deux à cet ensemble (par définition pour le premier couple, d'après le lemme 5.3 pour le second $)$. Donc $(\bar{\lambda}, \bar{\epsilon})=(\lambda, \epsilon)=\left(\lambda^{\max }, \epsilon^{\max }\right)$.

On suppose désormais $N>k(k+1) / 2$, donc $(\alpha, \beta) \neq(\emptyset, \emptyset)$. On utilise les constructions ci-dessus pour $u=\epsilon(1)$. On pose $v=u(-1)^{k}$. Nous voulons calculer le couple $\left(\lambda^{\max }, \epsilon^{\max }\right)$. Pour cela, on va calculer le couple $\left(\alpha^{\max }, \beta^{\max }\right)$ qui paramètre $\rho\left(\lambda^{\max }, \epsilon^{\max }\right)$. Comme on l'a vu dans la preuve de 4.5. c'est l'unique élément de $P_{k,-1-k ; 2}\left(\alpha, \beta,<_{\alpha, \beta, k}\right)$. Changer $(\alpha, \beta, k)$ en $\left(\alpha^{v}, \beta^{v}, k^{v}\right)$ ne change rien si $v=1$ et permute $\alpha$ et $\beta$ ainsi que toutes les données affectées à ces partitions si $v=-1$. Il en résulte que $\left(\alpha^{\max , v}, \beta^{\max , v}\right)$ est l'unique élément de $P_{k^{v},-1-k^{v} ; 2}\left(\alpha^{v}, \beta^{v},<_{\alpha^{v}, \beta^{v}, k^{v}}\right)$.

Introduisons les uniques bijections croissantes $\phi: \mathbb{N}_{>0} \rightarrow J^{u}$ et $\psi: \mathbb{N}_{>0} \rightarrow J^{-u}$. Les égalités (1) et (2) entraînent

(3) $\alpha_{j}^{v}=\lambda_{\phi(j)} / 2-k^{v}+2 j-\phi(j)-1, \beta_{j}^{v}=\lambda_{\psi(j)} / 2+k^{v}+2 j-\psi(j)$ pour tout $j \in \mathbb{N}_{>0}$.

Munissons l'ensemble d'indices de $\left(\alpha^{v}, \beta^{v}\right)$ d'un représentant $(n, m,<)$ de l'ordre $<_{\alpha^{v}, \beta^{v}, k^{v}}$. On suppose $n$ et $m$ assez grands. Par définition de l'ordre, on a

$$
\Leftrightarrow \lambda_{\phi(j)} / 2+1-\phi(j)>\lambda_{\psi(l)} / 2+1-\psi(l) \Leftrightarrow \phi(j)<\psi(l) .
$$

Puisque $1 \in J^{u}$, on a $\phi(1)=1$ donc $(1,0)<(1,1)$. Puisque $\left(\alpha^{v}, \beta^{v}\right) \neq(\emptyset, \emptyset)$, on $n_{<}>0$, cf. 1.2(1). L'unique élément de $P_{k^{v},-1-k^{v} ; 2}\left(\alpha^{v}, \beta^{v},<_{\alpha^{v}, \beta^{v}, k^{v}}\right)$ est créé par le procédé (a). Ce procédé crée des suites $a_{1}, a_{2}, \ldots, b_{1}, b_{2}, \ldots$, un nombre que nous notons $\boldsymbol{\nu}_{1}$ et un couple de partitions que nous notons $(\boldsymbol{\alpha}, \boldsymbol{\beta})$ extraites de $\left(\alpha^{v}, \beta^{v}\right)$. En notant $(\boldsymbol{\nu}, \boldsymbol{\mu})$ l'unique élément de $\mathcal{P}_{k^{v}-2,-1-k^{v} ; 2}(\boldsymbol{\alpha}, \boldsymbol{\beta},<)$, on a alors

$$
\begin{aligned}
& \alpha^{\max , v}+\left[k^{v},-\infty\left[_{2}=\left\{\boldsymbol{\nu}_{1}+k^{v}\right\} \sqcup\left(\boldsymbol{\nu}+\left[k^{v}-2,-\infty\left[_{2}\right),\right.\right.\right.\right. \\
& \beta^{\max , v}+\left[-k^{v}-1,-\infty\left[_{2}=\boldsymbol{\mu}+\left[-k^{v}-1,-\infty\left[_{2} .\right.\right.\right.\right.
\end{aligned}
$$

La description (4) de l'ordre permet de calculer les suites $a_{1}, a_{2}, \ldots$ et $b_{1}, b_{2}, \ldots$ : si on découpe $J^{u}$ et $J^{-u}$ en intervalles d'entiers consécutifs maximaux pour cette propriété, les images par $\phi$ des nombres $a_{1}, a_{2}, \ldots$ sont les premiers termes des intervalles de $J^{\epsilon(1)}$ tandis que les images par $\psi$ des nombres $b_{1}, b_{2}, \ldots$ sont les premiers termes des intervalles de $J^{-\epsilon(1)}$. D'après $\underline{5.2}(1)$, on a $\phi\left(a_{h}\right)=s_{2 h-1}$ et $\psi\left(b_{h}\right)=s_{2 h}$ pour tout $h \in \mathbb{N}_{>0}$. En utilisant (3), on obtient

$$
\begin{gathered}
\boldsymbol{\nu}_{1}=\left(\sum_{h \in \mathbb{N}_{>0}} \alpha_{a_{h}}^{v}\right)+\left(\sum_{h \in \mathbb{N}_{>0}} \beta_{b_{h}}^{v}\right) \\
=\left(\sum_{h \in \mathbb{N}_{>0}}\left(\lambda_{s_{2 h-1}} / 2-k^{v}+2 a_{h}-s_{2 h-1}-1\right)\right)+\left(\sum_{h \in \mathbb{N}_{>0}}\left(\lambda_{s_{2 h}} / 2+k^{v}+2 b_{h}-s_{2 h}\right)\right) .
\end{gathered}
$$

Il n'y a qu'un nombre fini de termes non nuls dans chacune de ces séries. Il en est de même des termes $\lambda_{s_{2 h-1}} / 2$ et $\lambda_{s_{2 h}} / 2$, que l'on peut donc sortir des séries. On 
regroupe le $h+1$-ième terme restant de la première série et le $h$-ième terme restant de la seconde. Il reste isolé le premier terme restant de la première série. On obtient $\boldsymbol{\nu}_{1}=\left(\sum_{h \in \mathbb{N}_{>0}} \lambda_{s_{h}} / 2\right)+\left(-k^{v}+2 a_{1}-s_{1}-1\right)+\left(\sum_{h \in \mathbb{N}_{>0}}\left(2 a_{h+1}-s_{2 h+1}-1+2 b_{h}-s_{2 h}\right)\right)$.

Le terme isolé vaut $-k^{v}$ puisque $a_{1}=s_{1}=1$. Pour $h \in \mathbb{N}_{>0}, a_{h+1}+b_{h}$ est le nombre d'éléments de la réunion de l'ensemble des éléments de $J^{u}$ inférieurs ou égaux à $s_{2 h+1}$ et de celui des éléments de $J^{-u}$ inférieurs ou égaux à $s_{2 h}$. Cet ensemble est la réunion de l'intervalle $\left\{1, \ldots, s_{2 h}\right\}$ et de $\left\{s_{2 h+1}\right\}$. Donc $a_{h+1}+b_{h}=s_{2 h}+1$. Le terme de la dernière série ci-dessus est donc $s_{2 h}+1-s_{2 h+1}$. Ceci est l'opposé du nombre d'éléments de $\tilde{J}^{-\epsilon(1)}$ compris entre $s_{2 h}$ et $s_{2 h+1}$, ou encore entre $s_{2 h-1}$ et $s_{2 h+1}$ puisque les termes de $\left\{s_{2 h-1}, \ldots, s_{2 h}-1\right\}$ ne sont pas dans $J^{-\epsilon(1)}$. La somme de la série est donc égale à $-\left|\tilde{J}^{-\epsilon(1)}\right|$. On obtient

$$
\boldsymbol{\nu}_{1}=\left(\sum_{h \in \mathbb{N}_{>0}} \lambda_{s_{h}} / 2\right)-k^{v}-\left|\tilde{J}^{-\epsilon(1)}\right| .
$$

En se rappelant la définition de $\bar{\lambda}_{1}$, cf. [5.1, on obtient

$$
\boldsymbol{\nu}_{1}+k^{v}=\bar{\lambda}_{1} / 2 .
$$

La partition $\boldsymbol{\alpha}$ est extraite de $\alpha^{v}$ en supprimant les termes $a_{1}, a_{2}, \ldots$. Puisque $\phi$ est une bijection de l'ensemble des indices de $\alpha^{v}$ sur $J^{u}$ et qu'elle envoie la suite $a_{1}, a_{2}, \ldots$ sur $J^{u} \cap \mathfrak{S}$, la partition $\boldsymbol{\alpha}$ est naturellement indexée par $\tilde{J}^{u}$. Posons $n^{\prime}=\left|\tilde{J}^{u}\right|$ et notons $\iota=\left\{1, \ldots, n^{\prime}\right\} \rightarrow \mathbb{N}_{>0}-\left\{a_{1}, a_{2}, \ldots\right\}$ l'unique bijection croissante. Alors

(7) $\boldsymbol{\alpha}_{j}=\alpha_{\iota(j)}^{v}$ pour $j \in\left\{1, \ldots, n^{\prime}\right\}$ et $\boldsymbol{\alpha}_{j}=0$ si $j \geq n^{\prime}+1$.

Pour $j \in\left\{1, \ldots, n^{\prime}\right\}$, on obtient grâce à $(3)$

$$
\boldsymbol{\alpha}_{j}=\lambda_{\phi \circ \iota(j)} / 2-k^{v}+2 \iota(j)-\phi \circ \iota(j)-1 .
$$

L'application $\phi \circ \iota$ est l'unique bijection croissante de $\left\{1, \ldots n^{\prime}\right\}$ sur $\tilde{J}^{u}$. On se rappelle le couple $\left(\lambda^{\prime}, \epsilon^{\prime}\right)$ de 5.1 et la bijection $\varphi:\left\{1, \ldots, r^{\prime}\right\} \rightarrow \tilde{J}^{+1} \sqcup \tilde{J}^{-1}$ de 5.2 . Cette bijection envoie $J^{\prime}-u \cap\left\{1, \ldots, r^{\prime}\right\}$ sur $\tilde{J}^{u}$. Posons $\phi^{\prime}=\varphi^{-1} \circ \phi \circ \iota$. Alors $\phi^{\prime}$ est l'unique bijection croissante de $\left\{1, \ldots, n^{\prime}\right\}$ sur $J^{\prime}-u \cap\left\{1, \ldots, r^{\prime}\right\}$. Soit $j \in\left\{1, \ldots, n^{\prime}\right\}$, posons $q=\phi^{\prime}(j)$ et soit $h \geq 1$ l'entier tel que $a_{h}<\iota(j)<a_{h+1}$. On a alors $\iota(j)=j+h$. On a aussi $s_{2 h-1}<\phi \circ \iota(j)<s_{2 h}$, c'est-à-dire $s_{2 h-1}<\varphi(q)<s_{2 h}$, donc $\varphi(q)=q+2 h-1$, c'est-à-dire $\phi \circ \iota(j)=q+2 h-1$. La formule (7) devient $\boldsymbol{\alpha}_{j}=\lambda_{\varphi(q)} / 2-k^{v}+2 j-q$. Puisque $\varphi(q) \in \tilde{J}^{u}=\tilde{J}^{\epsilon(1)}$, on a $\lambda_{\varphi(q)}=\lambda_{q}^{\prime}$. Posons $k^{\prime}=k\left(\lambda^{\prime}, \epsilon^{\prime}\right)$ et $v^{\prime}=u(-1)^{k^{\prime}+1}$. D'après 5.3(5), on a $k^{v}=k^{\prime} v^{\prime}+1$. La formule précédente se récrit $\boldsymbol{\alpha}_{j}+k^{\prime} v^{\prime}+2-2 j=\lambda_{\phi^{\prime}(j)}^{\prime} / 2+1-\phi^{\prime}(j)$. Quand $j$ décrit $\left\{1, \ldots, n^{\prime}\right\}$, les termes de droite décrivent les $n^{\prime}$ premiers termes de $A_{\lambda^{\prime}, \epsilon^{\prime}}^{-u}$, cela d'après (2) appliqué à $\left(\lambda^{\prime}, \epsilon^{\prime}\right)$. Donc les $n^{\prime}$ premiers termes des suites $\boldsymbol{\alpha}+\left[k^{\prime} v^{\prime},-\infty\left[2\right.\right.$ et $A_{\lambda^{\prime}, \epsilon^{\prime}}^{-u}$ coïncident. Montrons qu'il en est de même des termes suivants. Puisque ceux-ci décroissent de 2 en 2 , il suffit de prouver que les $n^{\prime}+1$-ièmes termes coïncident. Celui de la suite de gauche est $k^{\prime} v^{\prime}-2 n^{\prime}$. Celui de la suite de droite est $1-q^{\prime}$, où $q^{\prime}$ est le plus petit élément de $J^{\prime}-u$ strictement supérieur à $r^{\prime}$. Autrement dit, $q^{\prime}$ est le plus petit entier strictement supérieur à $r^{\prime}$ tel que $(-1)^{q^{\prime}+1}=-u$. L'assertion 5.2(6) dit que $(-1)^{r^{\prime}}=\epsilon(1)=u$. Donc $q^{\prime}=r^{\prime}+2$. On a aussi $k^{\prime} v^{\prime}=k^{v}-1=\left|\tilde{J}^{u}\right|-\left|\tilde{J}^{-u}\right|$ d'après $\left[5.3(5)\right.$ et $(6)$. On se rappelle que $n^{\prime}=\left|\tilde{J}^{u}\right|$ et que $r^{\prime}=\left|\tilde{J}^{u}\right|+\left|\tilde{J}^{-u}\right|$. On en 
déduit l'égalité cherchée $k^{\prime} v^{\prime}-2 n^{\prime}=1-q^{\prime}$. En conclusion, on a l'égalité

$$
\boldsymbol{\alpha}+\left[k^{\prime} v^{\prime},-\infty\left[{ }_{2}=A_{\lambda^{\prime}, \epsilon^{\prime}}^{-u} .\right.\right.
$$

Des calculs similaires conduisent à l'égalité

$$
\boldsymbol{\beta}+\left[-k^{\prime} v^{\prime}-1,-\infty\left[{ }_{2}=B_{\lambda^{\prime}, \epsilon^{\prime}}^{-u} .\right.\right.
$$

En comparant avec (1) on obtient que

(8) $\left(\boldsymbol{\alpha}^{v^{\prime}}, \boldsymbol{\beta}^{v^{\prime}}\right)$ est le couple de partitions paramétrant $\rho\left(\lambda^{\prime}, \epsilon^{\prime}\right)$.

Introduisons le couple $\left(\lambda^{\prime} \max , \epsilon^{\prime \max }\right)$ associé à $\left(\lambda^{\prime}, \epsilon^{\prime}\right)$. Montrons que

(9) la représentation $\rho\left(\lambda^{\prime} \max , \epsilon^{\prime \max }\right)$ est paramétrée par le couple de partitions $\left(\boldsymbol{\nu}^{v^{\prime}}, \boldsymbol{\mu}^{v^{\prime}}\right)$.

D'après (8) et la preuve de 4.5. $\rho\left(\lambda^{\prime} \max , \epsilon^{\prime \max }\right)$ est paramétrée par l'unique élément de $P_{k^{\prime},-1-k^{\prime} ; 2}\left(\boldsymbol{\alpha}^{v^{\prime}}, \boldsymbol{\beta}^{v^{\prime}},<_{\boldsymbol{\alpha}^{v^{\prime}}, \boldsymbol{\beta}^{v^{\prime}, k^{\prime}}}\right)$. Le couple $(\boldsymbol{\nu}, \boldsymbol{\mu})$ appartient à $P_{k^{v}-2,-1-k^{v} ; 2}(\boldsymbol{\alpha}, \boldsymbol{\beta},<)$. Or

$$
P_{k^{v}-2,-1-k^{v} ; 2}(\boldsymbol{\alpha}, \boldsymbol{\beta},<)=P_{k^{v}-1,-k^{v} ; 2}(\boldsymbol{\alpha}, \boldsymbol{\beta},<)=P_{k^{v}-1,-k^{v} ; 2}\left(\boldsymbol{\alpha}, \boldsymbol{\beta},<_{\boldsymbol{\alpha}, \boldsymbol{\beta}, k^{v}-1}\right),
$$

la première égalité provenant de 1.4(8), la deuxième du fait que l'on a vu dans la preuve de 2.11 que l'ordre $<$ induit de $<_{\alpha^{v}, \beta^{v}, k^{v}}$ était équivalent à l'ordre $<_{\boldsymbol{\alpha}, \boldsymbol{\beta}, k^{v}-1}$. Puisque $k^{v}-1=k^{\prime} v^{\prime}$, on obtient $(\boldsymbol{\nu}, \boldsymbol{\mu}) \in P_{k^{\prime} v^{\prime},-1-k^{\prime} v^{\prime} ; 2}\left(\boldsymbol{\alpha}, \boldsymbol{\beta},<_{\boldsymbol{\alpha}, \boldsymbol{\beta}, k^{\prime} v^{\prime}}\right)$. Par la même symétrie que l'on a utilisée plus haut, cela équivaut à $\left(\boldsymbol{\nu}^{v^{\prime}}, \boldsymbol{\mu}^{v^{\prime}}\right) \in$ $P_{k^{\prime},-1-k^{\prime} ; 2}\left(\boldsymbol{\alpha}^{v^{\prime}}, \boldsymbol{\beta}^{v^{\prime}},<_{\boldsymbol{\alpha}^{v^{\prime}}, \boldsymbol{\beta}^{v^{\prime}}, k^{\prime}}\right)$, ce qui démontre (9).

On applique le théorème 5.1 par récurrence : on a $\left(\lambda^{\prime} \max , \epsilon^{\prime \max }\right)=\left(\bar{\lambda}^{\prime}, \bar{\epsilon}^{\prime}\right)$. On utilise la relation (1), où l'on remplace $(\lambda, \epsilon)$ par $\left(\bar{\lambda}^{\prime}, \bar{\epsilon}^{\prime}\right)$ et $u$ par $-u$. Alors $v$ y est remplacé par $v^{\prime}$ et, d'après $(9),(\alpha, \beta)$ y est remplacé par $\left(\boldsymbol{\nu}^{v^{\prime}}, \boldsymbol{\mu}^{v^{\prime}}\right)$. On obtient

$$
\boldsymbol{\nu}+\left[k^{\prime} v^{\prime},-\infty\left[{ }_{2}=A_{\bar{\lambda}^{\prime}, \bar{\epsilon}^{\prime}}^{-u}, \boldsymbol{\beta}+\left[-1-k^{\prime} v^{\prime},-\infty\left[{ }_{2}=B_{\bar{\lambda}^{\prime}, \bar{\epsilon}^{\prime}}^{-u} .\right.\right.\right.\right.
$$

D'après (2), on a

$$
A_{\bar{\lambda}^{\prime}, \bar{\epsilon}^{\prime}}^{-u}=\left\{\bar{\lambda}_{j}^{\prime} / 2+1-j ; j \geq 1,(-1)^{j+1} \bar{\epsilon}^{\prime}(j)=-u\right\} .
$$

Par construction, on a $\bar{\lambda}_{j}^{\prime}=\bar{\lambda}_{j+1}^{\prime}, \bar{\epsilon}^{\prime}(j)=\bar{\epsilon}(j+1)$. D'où

$$
A_{\bar{\lambda}^{\prime}, \bar{\epsilon}^{\prime}}^{-u}=\left\{\bar{\lambda}_{j} / 2+2-j ; j \geq 2,(-1)^{j+1} \bar{\epsilon}(j)=u\right\} .
$$

En se rappelant que $k^{\prime} v^{\prime}=k^{v}-1$, on obtient

$$
\boldsymbol{\nu}+\left[k^{v}-2,-\infty\left[{ }_{2}=\left\{\bar{\lambda}_{j} / 2+1-j ; j \geq 2,(-1)^{j+1} \bar{\epsilon}(j)=u\right\} .\right.\right.
$$

Autrement dit, $\boldsymbol{\nu}+\left[k^{v}-2,-\infty\left[{ }_{2}\right.\right.$ est l'ensemble $A_{\bar{\lambda}, \bar{\epsilon}}^{u}$ dont on supprime le premier terme. Par construction, $\bar{\epsilon}(1)=\epsilon(1)$. Grâce à $(6), \boldsymbol{\nu}_{1}+k^{v}$ n'est autre que le premier terme de $A_{\bar{\lambda}, \bar{\epsilon}}^{u}$. Grâce à (5), on obtient

$$
\alpha^{\max , v}+\left[k^{v},-\infty\left[{ }_{2}=A_{\bar{\lambda}, \bar{\epsilon}}^{u} .\right.\right.
$$

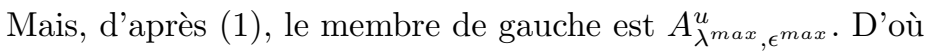

$$
A_{\lambda^{\max }, \epsilon^{\max }}^{u}=A_{\bar{\lambda}, \bar{\epsilon}}^{u} .
$$

On démontre de même

$$
B_{\lambda^{\max }, \epsilon^{\max }}^{u}=B_{\bar{\lambda}, \bar{\epsilon}}^{u} .
$$

Ces deux égalités entraînent $\left(\lambda^{\max }, \epsilon^{\max }\right)=(\bar{\lambda}, \bar{\epsilon})$. Cela démontre le théorème 5.1 


\section{RÉFÉRENCES}

[1] George Lusztig, Character sheaves. V, Adv. in Math. 61 (1986), no. 2, 103-155, DOI 10.1016/0001-8708(86)90071-X. MR849848

[2] G. Lusztig, Cuspidal local systems and graded Hecke algebras. III, Represent. Theory 6 (2002), 202-242, DOI 10.1090/S1088-4165-02-00172-3. MR1927954

[3] I. G. Macdonald, Symmetric functions and Hall polynomials, The Clarendon Press, Oxford University Press, New York, 1979. Oxford Mathematical Monographs. MR553598

[4] Toshiaki Shoji, Green functions associated to complex reflection groups, J. Algebra 245 (2001), no. 2, 650-694, DOI 10.1006/jabr.2001.8898. MR.1863896

[5] Jean-Loup Waldspurger, Représentations de réduction unipotente pour $\mathrm{SO}(2 n+1)$ : quelques conséquences d'un article de Lusztig (French), Contributions to automorphic forms, geometry, and number theory, Johns Hopkins Univ. Press, Baltimore, MD, 2004, pp. 803-910. $\operatorname{MR} 2058629$

CNRS-Institut de Mathématiques de Jussieu-PRG, 4 Place Jussieu, Boîte Courrier 247, 75252 PARIS CEDEX 05

Email address: jean-loup.waldspurger@imj-prg.fr 